\title{
STABLE HIGH CONDUCTIVITY BILAYERED ELECTROLYTES FOR LOW TEMPERATURE SOLID OXIDE FUEL CELLS
}

FETC AAD Document Control Bldg. 921

\author{
U.S. Department of Energy
}

Federal Energy Technology Center

P.O. Box 10940

Pittsburgh, PA 15236-0940

Contract \# DE-AC26-99FT40712

Program Manager: Lane Wilson

Final Report: Period 10/1/99 - 9/30/02

Eric D. Wachsman and Keith L. Duncan

Department of Materials Science and Engineering

University of Florida

Gainesville, FL 32611-6400 


\section{DISCLAIMER}

"This report was prepared as an account of work sponsored by an agency of the United States Government. Neither the United States Government nor any agency thereof, nor any employees, makes any warranty, express or implied, or assumes any legal liability or responsibility for the accuracy, completeness, or usefulness of any information, apparatus, product, or process disclosed, or represents that its use would not infringe privately owned rights. Reference herein to any specific commercial product, process, or service by trade name, trademark, manufacturer, or otherwise does not necessarily constitute or imply its endorsement, recommendation, or favoring by the United States Government or any agency thereof. The views and opinions of the authors expressed herein do not necessarily state or reflect those of the United States Government or any agency thereof." 


\begin{abstract}
A bilayer electrolyte consisting of acceptor-doped ceria (on the fuel/reducing side) and cubicstabilized bismuth oxide (on the oxidizing side) was developed. The bilayer electrolyte that was developed showed significant improvement in open-circuit potential versus a typical ceria based SOFC. Moreover, the OCP of the bilayer cells increased as the thickness of the bismuth oxide layer increased relative to the ceria layer. Thereby, verifying the bilayer concept. Although, because of the absence of a suitable cathode (a problem we are still working assiduously to solve), we were unable to obtain power density curves, our modeling work predicts a reduction in electrolyte area specific resistance of two orders of magnitude over cubic-stabilized zirconia and projects a maximum power density of $9 \mathrm{~W} / \mathrm{m}^{2}$ at $800{ }^{\circ} \mathrm{C}$ and $0.09 \mathrm{~W} / \mathrm{m}^{2}$ at $500{ }^{\circ} \mathrm{C}$.

Towards the development of the bilayer electrolyte other significant strides were made. Among these were, first, the development of a, bismuth oxide based, oxide ion conductor with the highest conductivity $\left(0.56 \mathrm{~S} / \mathrm{cm}\right.$ at $800{ }^{\circ} \mathrm{C}$ and $0.043 \mathrm{~S} / \mathrm{cm}$ at $\left.500{ }^{\circ} \mathrm{C}\right)$ known to date. Second, a physical model of the defect transport mechanisms and the driving forces for the ordering phenomena in bismuth oxide and other fluorite systems was developed. Third, a model for point defect transport in oxide mixed ionic-electronic conductors was developed, without the typical assumption of a uniform distribution of ions and including the effect of variable loads on the transport properties of an SOFC (with either a single or bilayer electrolyte).
\end{abstract}




\section{CONTENTS}

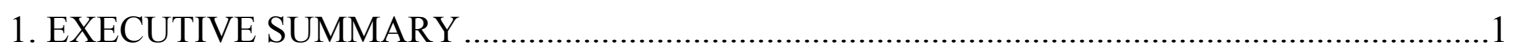

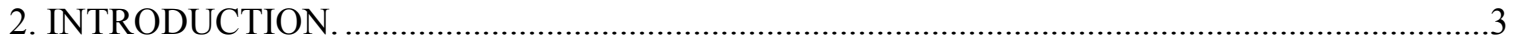

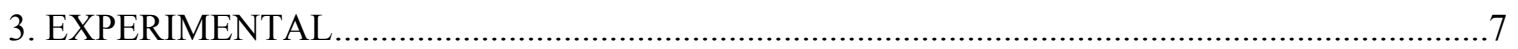

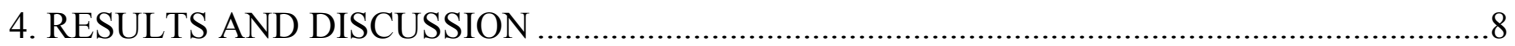

4.1 Synthesis and Characterization of Doped Ceria Electrolytes ................................................

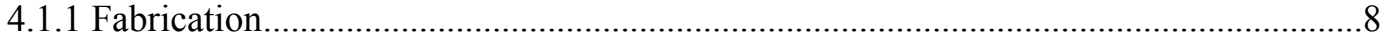

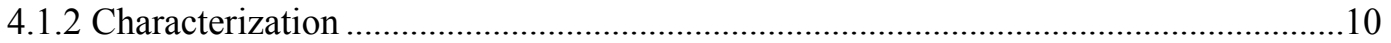

4.2 Synthesis, Characterization and Optimization of Bismuth Oxide ........................................17

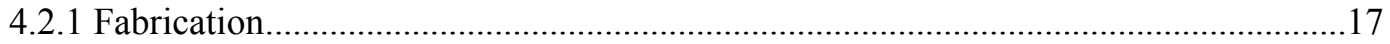

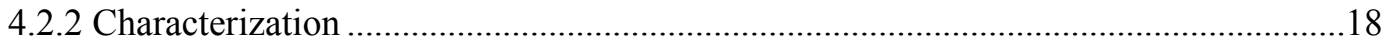

4.2.3 Neutron Diffraction Study of Occupancy and Positional Order of Oxygen Ions ........19

4.2.4 Effect of Dopant Polarizability on Oxygen Sublattice Order.......................................27

4.2.5 Effect of Oxygen Sublattice Ordering on Interstitial Transport Mechanism................33

4.2.6 Development of Highest Conductivity $\mathrm{Bi}_{2} \mathrm{O}_{3}$-Based Electrolyte..................................47

4.2.7 Structural Stability and Conductivity of Cubic $\left(\mathrm{WO}_{3}\right)_{\mathrm{x}}-\left(\mathrm{Dy}_{2} \mathrm{O}_{3}\right)_{\mathrm{y}}-\left(\mathrm{Bi}_{2} \mathrm{O}_{3}\right)_{1-\mathrm{x}-\mathrm{y}} \ldots \ldots \ldots \ldots . . . . .54$

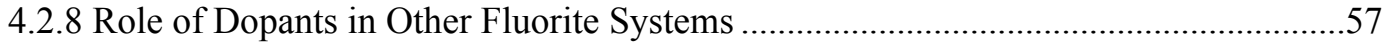

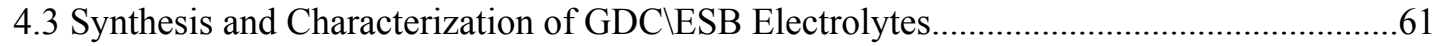

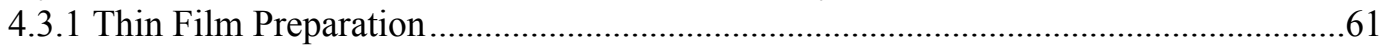

4.3.2 Characterization of Bilayer SDC $\backslash$ ESB and GDC $\backslash$ ESB Electrolytes ...........................63

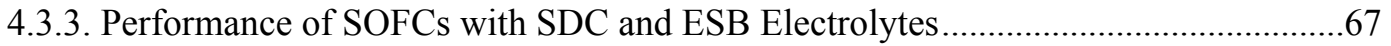

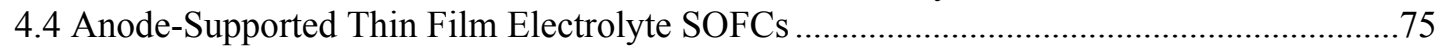

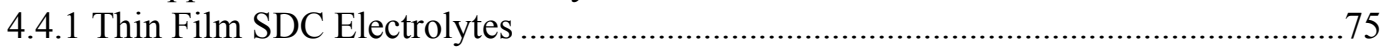

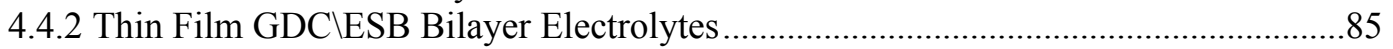

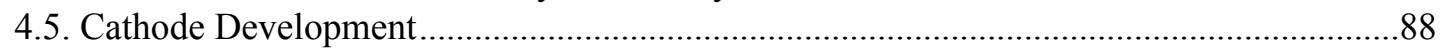

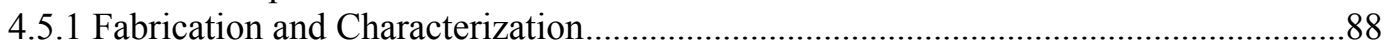

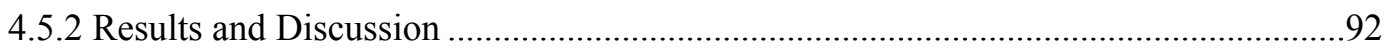

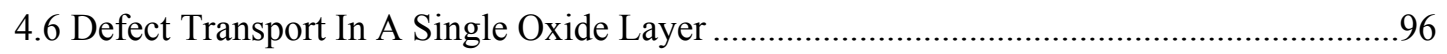

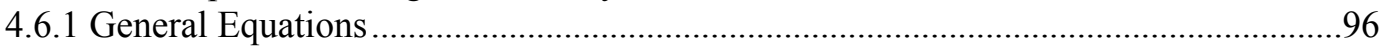

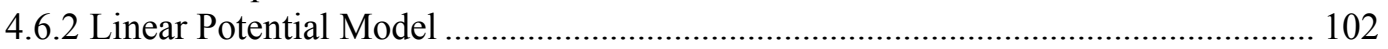

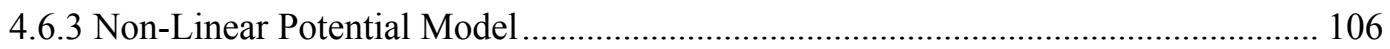

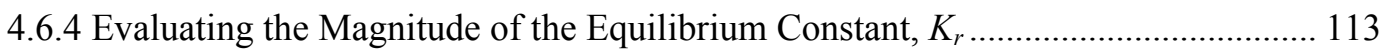

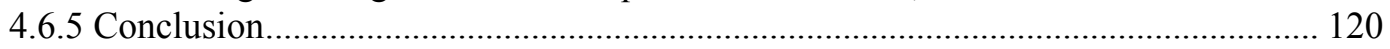

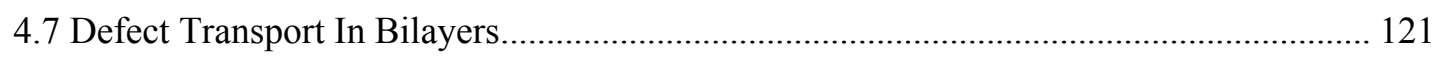

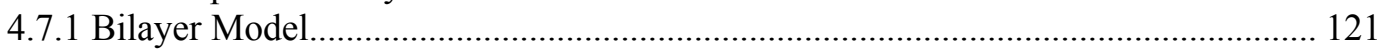

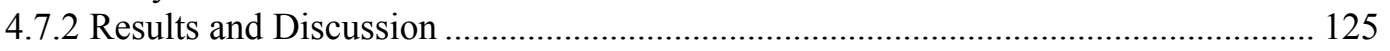

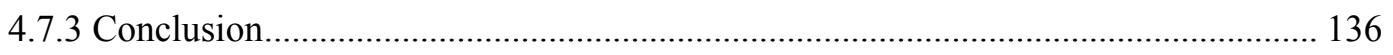

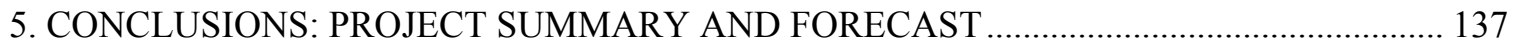

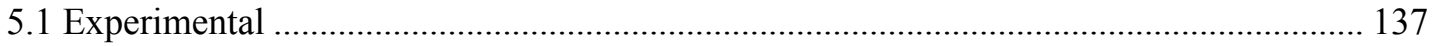

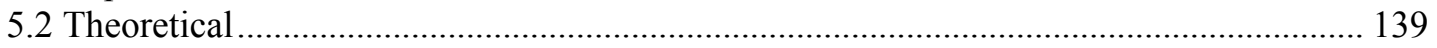

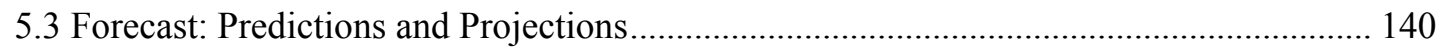

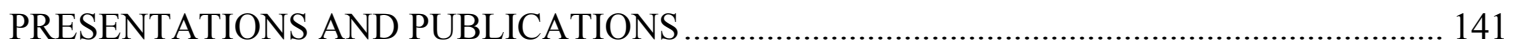

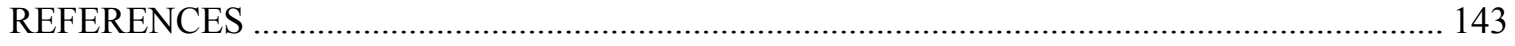




\section{EXECUTIVE SUMMARY}

This report provides a synopsis of our effort to develop a low temperature SOFC with a bilayer electrolyte of acceptor-doped ceria and stabilized bismuth oxide. As the report will show we have made significant strides towards this reality, but there is still more ground to gain.

Our research started, fittingly, with a four-pronged approach. This consisted of tackling some of the challenges remaining in the development and performance of the acceptor-doped ceria and stabilized-bismuth oxide, namely the sinterability of the former (section 4.1) and the ordering phenomena in the later (section 4.2), before putting them together for the bilayer. Simultaneously, we also worked on perfecting our SOFC/concentration cell setups and began the theoretical work on the bilayer (sections 4.6 and 4.7).

After obtaining satisfactory results in processing ceria samples the next logical step was cell fabrication. The high sintering temperature of ceria and the importance of reducing polarization losses led to the decision to use anode-supported SOFC architecture. To do so we explored dip-coating and pulse laser deposition as potential avenues for this (sections 4.3 and 4.4). The former was found to be the most convenient and practical approach for our resources and we were able to deposit satisfactory ceria films on Ni-ceria anodes for our cells. Moreover, the results obtained from our ceria SOFCs were comparable to those reported in the literature.

Our work on point-defect ordering in stabilized bismuth oxide allowed us to gain physical insight into the defect transport mechanisms and the driving forces for the ordering phenomena not only in bismuth oxide but also other fluorite systems (section 4.2). Although this was pioneering, basic research, the crowning technological achievement arising from this work was the development of a, bismuth oxide based, oxide ion conductor with the highest conductivity $\left(0.56 \mathrm{~S} / \mathrm{cm}\right.$ at $800{ }^{\circ} \mathrm{C}$ and $0.043 \mathrm{~S} / \mathrm{cm}$ at $500{ }^{\circ} \mathrm{C}$ ) known to date (section 4.2).

We assembled and tested the open-circuit potential of bilayered samples of various thickness ratios (section 4.3). The results showed, as expected, that the OCP approached the theoretical value as the thickness of the bismuth oxide layer increased relative to the thickness of the ceria layer. Thus validating the bilayer concept. In doing these experiments however, the critical need for suitable, high performance cathodes to interface with the bismuth oxide layer, became apparent. Much effort was done to find a suitable cathode (section 4.5), but time ran out before this could be completed. 
The lack of a suitable cathode hindered our ability to obtain current-voltage characteristics and power density curves for the bilayer cells. This work must be continued and will provide the final pieces needed to usher in a new age of low temperature solid oxide fuel cells.

Finally, our modeling work (sections 4.6 and 4.7) continued in parallel with the experiments, and here we were able to demonstrate theoretically how the bilayer based SOFC could operate stably as a function of operating conditions (e.g., temperature and load resistance). The model predicts a reduction in electrolyte area specific resistance of two orders of magnitude over cubic-stabilized zirconia and commensurate improvements in power density (chapter 5). To wit, the results predict that with a ceria-bismuth oxide bilayer electrolyte the limiting resistance would come from the electrodes! Specifically, the projected maximum power density at $800{ }^{\circ} \mathrm{C}\left(9 \mathrm{~W} / \mathrm{m}^{2}\right)$ is an order of magnitude greater than possible with the best YSZ electrolytes. At $500{ }^{\circ} \mathrm{C}$ the maximum power density $(0.1$ $\mathrm{W} / \mathrm{m}^{2}$ ) is comparable to that attained with YSZ SOFCs, using state of the art cathodes, at $600{ }^{\circ} \mathrm{C}$.

There is still more work to be done, this is undeniable, but equally irrefutable is the potential and emergent reality of low temperature SOFCs based on ceria-bismuth oxide bilayers. 


\section{INTRODUCTION}

\section{Background}

Solid oxide fuel cells (SOFCs) are the future of energy production. They offer great promise as a clean and efficient process for directly converting chemical energy to electricity while providing significant environmental benefits (they produce negligible hydrocarbons, $\mathrm{CO}$, or $\mathrm{NO}_{\mathrm{x}}$ and, as a result of their high efficiency, produce about one-third less $\mathrm{CO}_{2}$ per kilowatt hour than internal combustion engines).

Unfortunately, the current SOFC technology, based on a stabilized zirconia electrolyte, must operate in the region of $1000^{\circ} \mathrm{C}$ to avoid unacceptably high ohmic losses. These high temperatures demand (a) specialized (expensive) materials for the fuel cell interconnects and insulation, (b) time to heat up to the operating temperature and (c) energy input to arrive at the operating temperature. Therefore, if fuel cells could be designed to give a reasonable power output at low to intermediate ${ }^{1}$ temperatures tremendous benefits may be accrued. At low temperatures, in particular, it becomes feasible to use ferritic steel for interconnects instead of expensive and brittle ceramic materials such as those based on $\mathrm{LaCrO}_{3}$. In addition, sealing the fuel cell becomes easier and more reliable; rapid startup is facilitated; thermal stresses (e.g., those caused by thermal expansion mismatches) are reduced; radiative losses $\left(\sim \mathrm{T}^{4}\right)$ become minimal; electrode sintering becomes negligible and (due to a smaller thermodynamic penalty) the SOFC operating cycle (heating from ambient) would be more efficient. Combined, all these improvements further result in reduced initial and operating costs. The problem is, at lower temperatures the conductivity of the conventional stabilized zirconia electrolyte decreases to the point where it cannot supply electrical current efficiently to an external load, see Fig. 2.1.

The primary objective of the proposed research is to develop a stable high conductivity $(>0.05 \mathrm{~S}$ $\mathrm{cm}^{-1}$ at $\leq 550{ }^{\circ} \mathrm{C}$ ) electrolyte for lower temperature SOFCs, which is the lowest (and most difficult) temperature criteria for the $21^{\text {st }}$ Century Fuel Cell Program. Meeting this objective provides a potential for future transportation applications of SOFCs, where their ability to directly use hydrocarbon fuels could permit refueling within the existing transportation infrastructure.

\footnotetext{
${ }^{1}$ In accordance with current convention, the term "low" is used here to for temperatures below $600{ }^{\circ} \mathrm{C}$ while the term "intermediate" refers to temperatures in the range $600-800{ }^{\circ} \mathrm{C}$.
} 


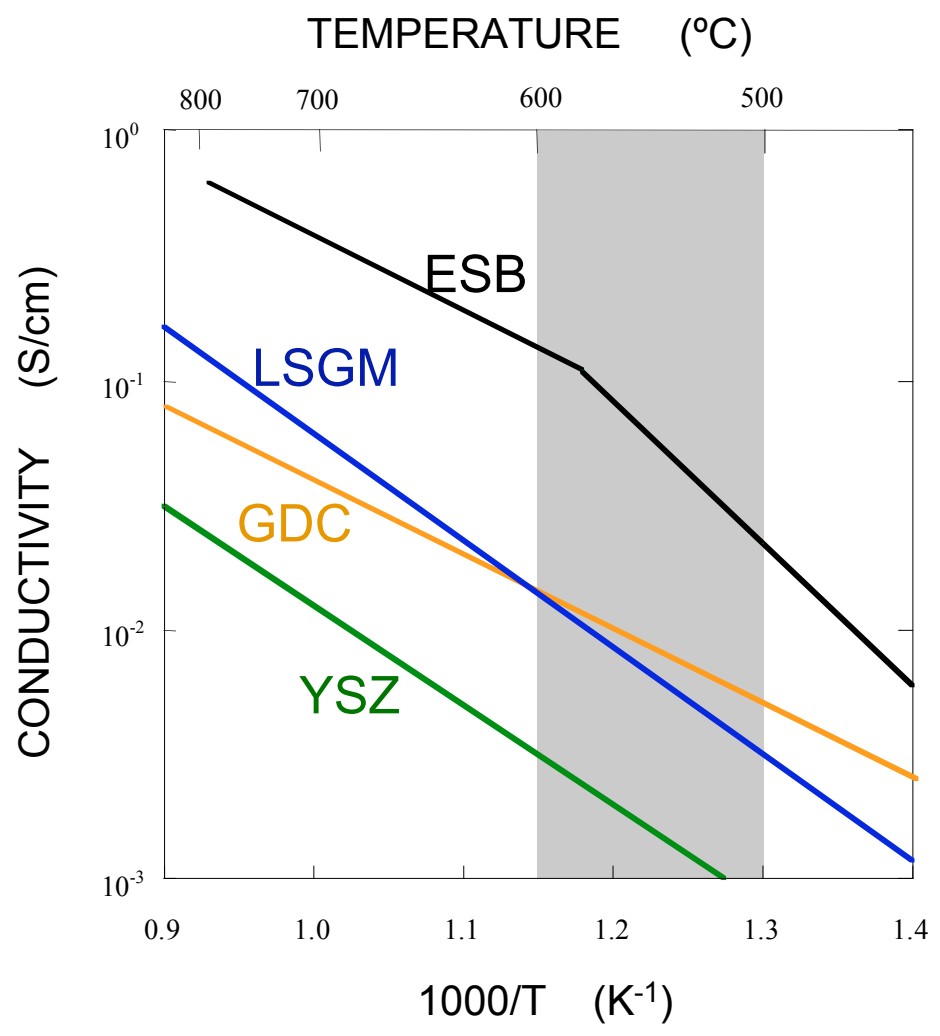

Figure 2.1. Comparison of the conductivity of candidate solid oxide electrolytes for SOFCs

\section{Concept}

Bismuth oxide and acceptor-doped ceria are among the best oxide ion conductors known and bismuth oxide based electrolytes are the only solid oxide electrolytes to have an ionic conductivity that meets the program conductivity goal. The problem is that in reducing conditions acceptor-doped ceria becomes a mixed conductor and bismuth oxide decomposes.

In order to produce a stable high conductivity electrolyte for low temperature SOFCs, we have proposed a functionally gradient bilayer electrolyte comprised of a layer of erbia-stabilized bismuth oxide (ESB) on the oxidizing side and a layer of samaria- or gadolinia-doped ceria (SDC or GDC) on the reducing side, as shown in Fig. 2.2. In this bilayer arrangement, the ceria layer is able to protect the bismuth oxide layer from decomposing by shielding it from very low $P_{\mathrm{O}_{2}}$ 's, if the constituent layers have the appropriate relative thickness. In addition, the ESB layer blocks electronic flux through the electrolyte. Thus, at worst, an SDC $\backslash$ ESB bilayer with a relatively thin layer of ESB should have the high conductivity of SDC but with improved efficiency due to the electronic flux barrier provided by ESB. At best, the conductivity of the bilayer should approach that of ESB with a relatively thick layer of ESB. 


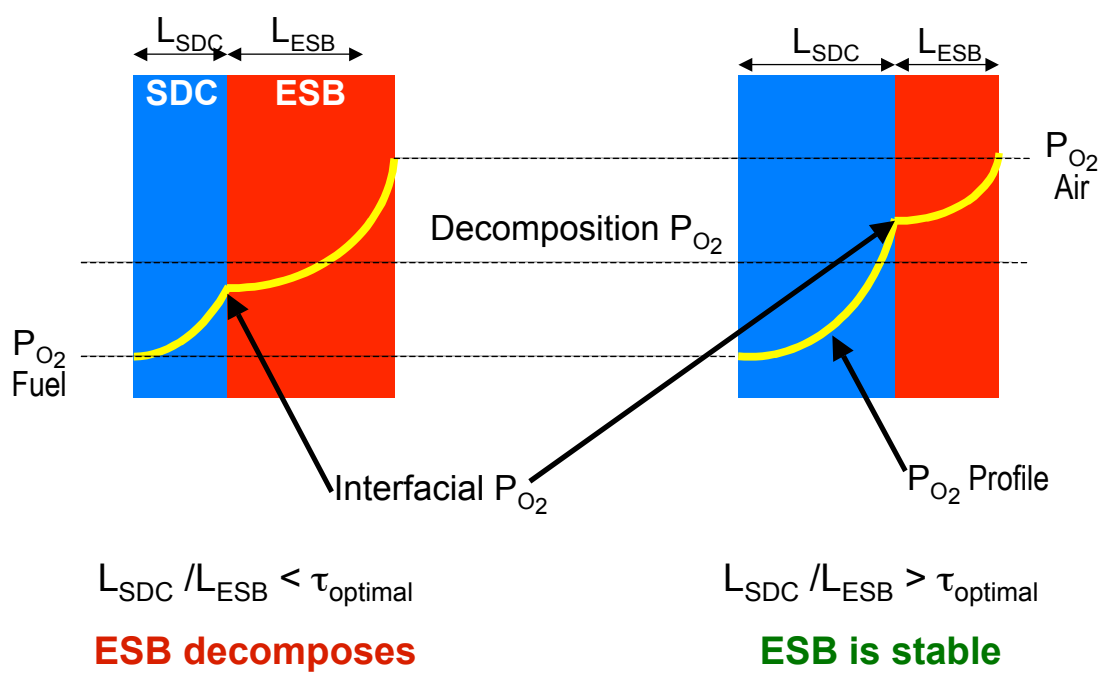

Figure 2.2. Conceptual representation of a SDC $\triangle E S B$ bilayered electrolyte showing the how the relative thickness can be varied to control the interfacial $P_{\mathrm{O}_{2}}$ to protect the ESB from decomposition.

The high conductivity of ESB is a significant advantage over the YSZ ISDC bilayers investigated by others $[1,2]$. Moreover, it has been shown that small (dopant) concentrations of SDC in ESB or ESB in SDC, have conductivities comparable to the host lattice [3, 4]. Therefore, if solid solutions are formed at the SDC-ESB interface, it should not be detrimental to the performance of the bilayer. In contrast, solid solutions of SDC and YSZ have been found to be significantly less conductive than SDC or YSZ. Thus, it bears emphasizing that, at this time, only SDC\ESB electrolytes have potential in low temperature SOFC applications.

We demonstrated previously that this concept works; that a bismuth oxide/ceria bilayer electrolyte provides near theoretical open circuit potential (OCP) and is stable for $1400 \mathrm{~h}$ of fuel cell operation under both open circuit and maximum power conditions [5]. In this project we extended the demonstration of the concept significantly, as described below.

\section{Completed Tasks}

In this report, the fabrication and characterization of single-layered electrolytes of SDC, ESB and bilayered electrolytes of SDC\ESB are presented as well as the fabrication of anode and cathode materials. Fabrication techniques include standard ceramic processing and the Citrate Process (CP) to produce powders; colloidal deposition and pulsed laser deposition (PLD) to produce thin film layers, screen printing for cathodes application; and uniaxial pressing and cold isostatic pressing (CIP) to produce substrates. Characterization techniques include x-ray diffraction, density analysis, scanning electron microscopy (SEM), x-ray photo-electronic spectroscopy (XPS), energy dispersive 
spectroscopy (EDS) and electrical impedance spectroscopy (EIS) and current-voltage (I-V) measurements.

A complete treatment of cubic-stabilized bismuth oxides is reported, including their structural properties and dopant polarizability and their effect on aging phenomena and conductivity. Significantly, our study of bismuth oxide culminates in the development of a new cubic stabilized bismuth oxide with the highest known oxide ion conductivity.

Finally, the report contains a defect transport model and a bilayer model (an application of the defect transport model), which are used to predict the dependence of bilayer stability and performance on the relative thickness of its constituent layers. 


\section{EXPERIMENTAL}

Because of the breadth and volume of this project it would have been illogical and disharmonious to assemble all the experimental procedure in this section. Hence, the numerous experiments conducted during the project may be found in the project subtopics as outlined in the Table of Contents. A summary of the pertinent sections is provided below.

EXPERIMENTAL

page(s)

4.1.1 Fabrication and Characterization of Doped Ceria Electrolytes ................................ $\quad$ 8-10

4.2.1 Fabrication and Characterization of Bismuth Oxide Electrolytes ............................ 17-19

4.2.3 Neutron Diffraction Study of Occupancy and Positional Order of Oxygen Ions ...... 19-27

4.2.4 Development of Highest Conductivity $\mathrm{Bi}_{2-(x+y)} \mathrm{Dy}_{x} \mathrm{~W}_{y} \mathrm{O}_{3}$ Electrolyte ....................... 47-49

4.3.1 Thin Film Preparation of Bilayer SDC\ESB and GDC $\mid$ ESB Electrolytes ................ 61-62

4.3.2 Characterization of Bilayer SDC LESB and GDC IESB Electrolytes ........................

4.4.1 Fabrication and Characterization of Anode-Supported Thin Film Electrolyte SOFCs 76-77

4.5.1 Fabrication and Characterization of Cathodes for Bilayer Electrolytes.................... 8 88-92 


\section{RESULTS AND DISCUSSION}

\subsection{Synthesis and Characterization of Doped Ceria Electrolytes}

In this section, the fabrication and characterization of samaria- and gadolinia-doped ceria (SDC and GDC) is reported. Several routes for fabricating SDC and GDC samples were explored in order to maximize the density of the samples and lower the sintering temperatures. The samples were then characterized through the usual analytical techniques to ensure they had the correct lattice structure, suitable morphology and low impurities levels. Finally, the conductivity of the samples was measured and the impact of the fabrication routes was assessed.

\subsubsection{Fabrication}

Solid-State Synthesis. SDC samples were prepared from polycrystalline powders of ceria and samaria. The powders were weighed to give the appropriate mole fractions and mechanically milled with zirconia media in ethanol for 24 hours. SDC powders were calcined at 1100,1200 and $1300^{\circ} \mathrm{C}$ for $10 \mathrm{hrs}$ and sintered at $1465{ }^{\circ} \mathrm{C}, 1550{ }^{\circ} \mathrm{C}$, and $1650{ }^{\circ} \mathrm{C}$ for $15 \mathrm{hrs}$. Then they were pulverized using a mortar and pestle and sieved (325 mesh). GDC powder was purchased directly from Rhodia. Theoretical densities were determined from the lattice parameter obtained with a Philips APD 3720 Xray diffractometer, and the relative density of sintered bodies was determined by Archimedes' method.

The densities of the SDC pellets prepared were found to be $70-98 \%$ of the theoretical density depending on the sintering temperature. Figure 4.1.1 shows the measured relative densities of SDC samples as a function of axially applied pressure by changing sintering temperature, calcination temperature, binder, and the use of cold-isostatic pressing (CIP). The density, $\rho$, of the sintered samples increased with the sintering temperature. Fabrication of SDC pellets by the solid-state technique required a fairly high temperature of $1600{ }^{\circ} \mathrm{C}$ in order to get more than $95 \%$ theoretical density. However, sintering at these temperatures is expensive and makes co-firing doped ceria with other materials difficult due to severe solid-state reaction and inter-diffusion at those temperatures [6]. Thus, it would be beneficial to fabricate doped ceria electrolyte at the lowest temperature as possible.

GDC powders were uniaxially (1450 psi) and isostatically (200 $\mathrm{MPa})$ pressed into disks (diameter $<25 \mathrm{~mm}$ and thickness $<2 \mathrm{~mm}$ ). The GDC disks were then sintered at $1650{ }^{\circ} \mathrm{C}$ and 1550 ${ }^{\circ} \mathrm{C}$, respectively, for $10 \mathrm{hrs}$. 


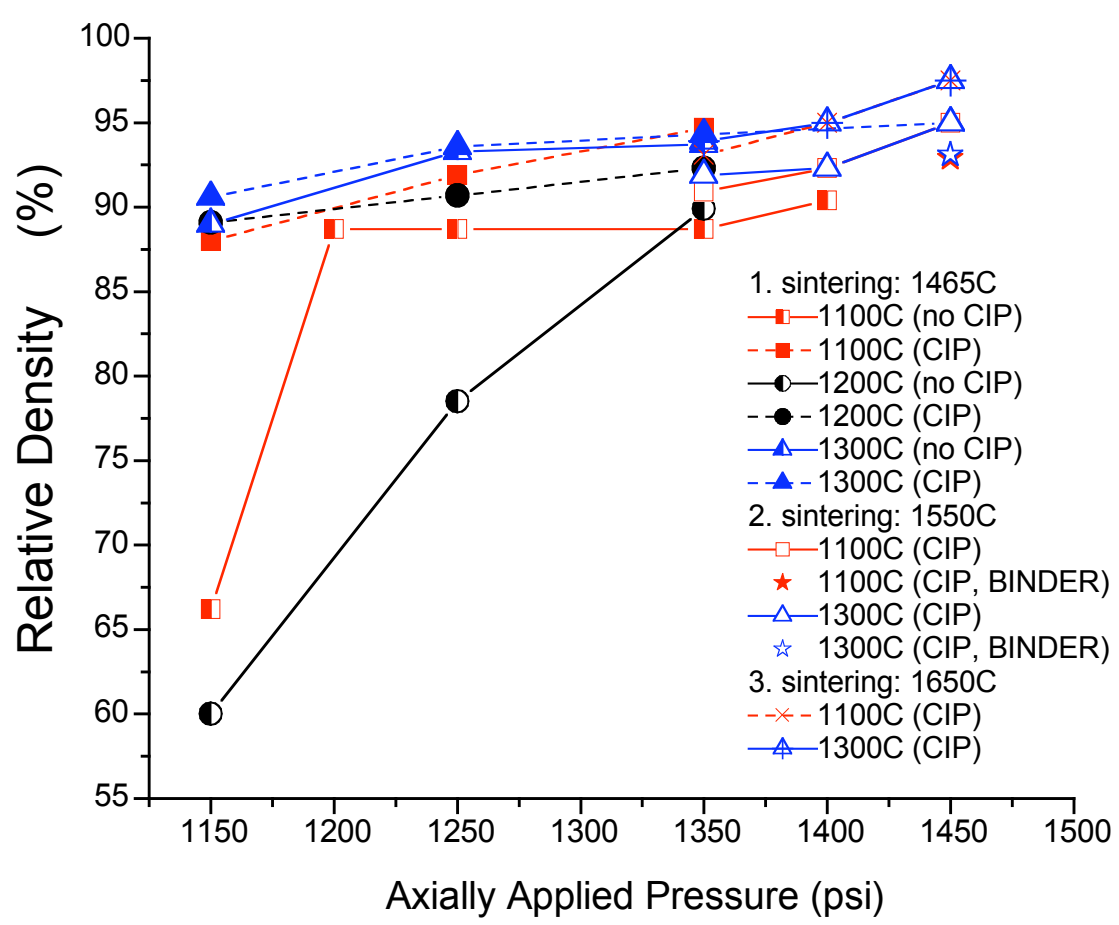

Figure 4.1.1. Effect of axially and isostatically pressing, calcining and sintering temperature, and binder (P.E.G. 8000) on SDC pellets.

Theoretically, denser and/or stronger green-bodies may be obtained through one or a combination of: increased pressing load, binder (typical organic liquids) reinforcement or isostatic pressing. All three methods were tested in an attempt to simultaneously increase the density and lower the sintering temperature of SDC samples.

The uniaxial pressing load was increased systematically for SDC samples, Fig. 4.1.1. However, it was found that increasing the uniaxial pressing load above 1500 psi causes de-lamination and end capping - two of the more common defects in die-compacted powders [7].

The binder polyethylene glycol (PEG) 8000 was tried in an attempt to increase the green-body strength. However, it was found (rather unexpectedly) that after sintering, the densities of the samples prepared with the PEG binder were lower than those prepared without it. This result was surprising because the PEG binder had increased the observed strength of the green-body after compaction (pressing). The reduction in sintered sample density may be attributable to structural anisotropy introduced by the binder that resulted in voids formation during sintering.

The highest SDC sample densities were achieved with cold-isostatically pressed samples. Coldisostatic pressing increased the densities of the sintered samples because the pressing load is uniform and, consequently, powder movement (which causes de-lamination) is reduced [7]. 
Citrate Process $(C P)$. Ceria-based ceramics synthesized by citrate process employed hydrated nitrate salts, $\mathrm{Ce}\left(\mathrm{NO}_{3}\right)_{3} \cdot 6 \mathrm{H}_{2} \mathrm{O}, \mathrm{Sm}\left(\mathrm{NO}_{3}\right)_{3} \cdot 6 \mathrm{H}_{2} \mathrm{O}$, and $\mathrm{Gd}\left(\mathrm{NO}_{3}\right)_{3} \cdot 6 \mathrm{H}_{2} \mathrm{O}(99.95 \%)$ from Kanto Chemical. The reactants were dissolved in de-ionized (DI) water then ethylene glycol and citric acid were added as the solvent and the metal chelating agent, respectively. The solution was slowly heated on a hot plate and carefully monitored until the DI water evaporated. At this point, the thermo-chemical reaction between nitrate salts occurred, and a precursor foam was produced. This foam was then fired at $600{ }^{\circ} \mathrm{C}$ for $2 \mathrm{hrs}$ to form SDC and GDC powders with fluorite structure. For microstructural analysis, the powders were examined using a JSM-35CF scanning electron microscope, which also performed energy dispersive analysis (EDS), a BET (Quantachrome Corp.), and an LS Particle Size Analyzer (Beckman-Coulter).

\subsubsection{Characterization}

Powders

Figure 4.1.2 shows representative XRD patterns of powders obtained by both (conventional) solid-state synthesis (SSS) and citrate process (CP). Powders fabricated by SSS are identical to those by $\mathrm{CP}$, and confirm the formation of cubic SDC and GDC. SEM micrographs in Fig. 4.1.3 show the powders synthesized by CP have more faceted polyhedral morphologies, whereas particles by SSS exhibited elliptical morphologies. The average particle size, measured by TEM micrographs and particle size

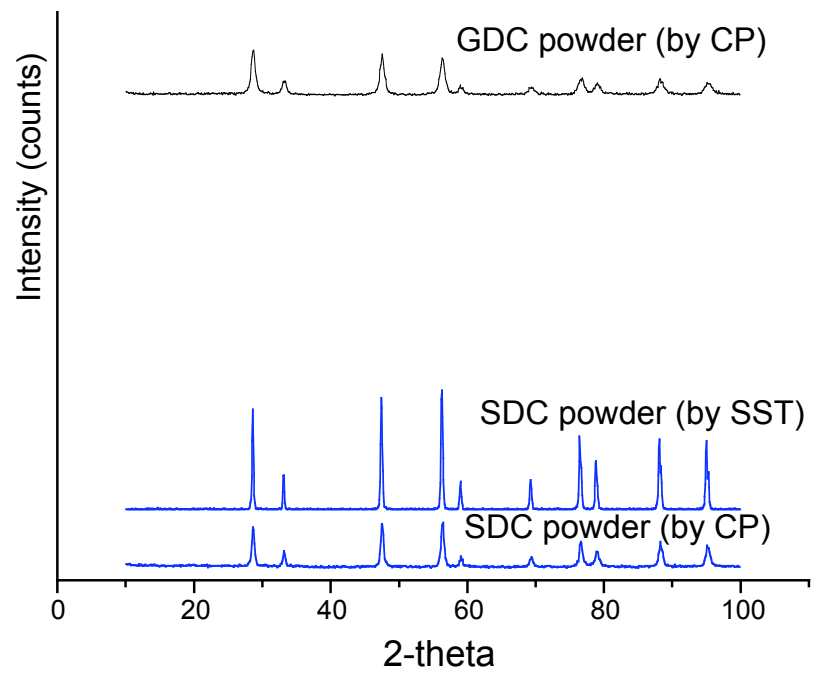

Figure 4.1.2. XRD patterns of calcined SDC, GDC, and ESB powder fabricated by SSS and CP 
(a)

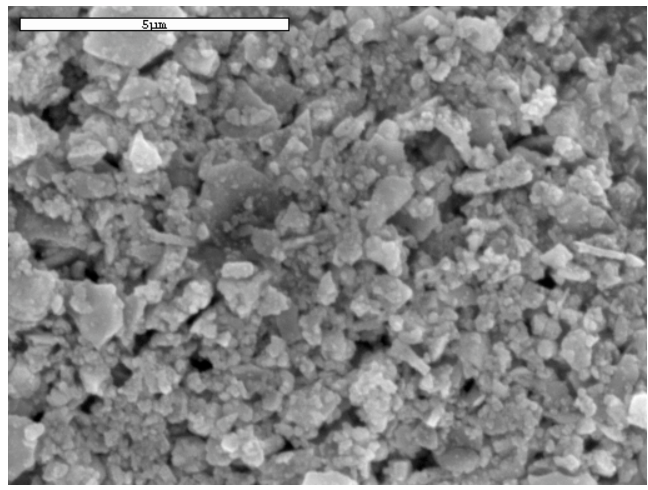

(c)

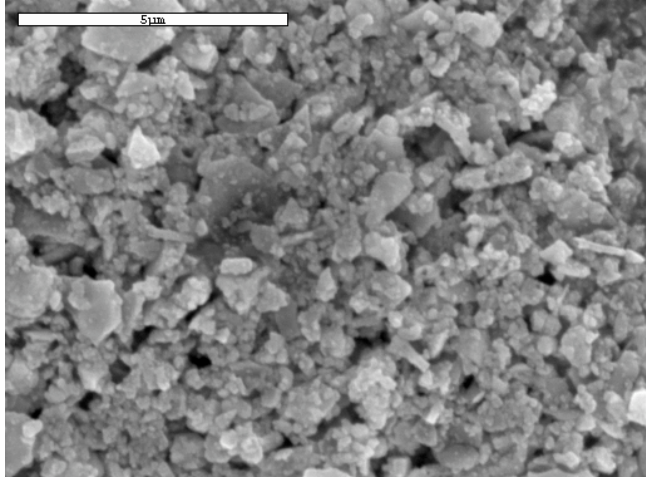

(b)

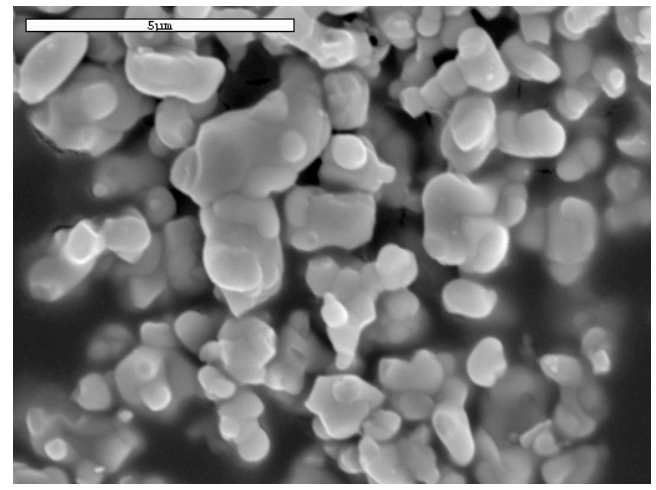

(d)

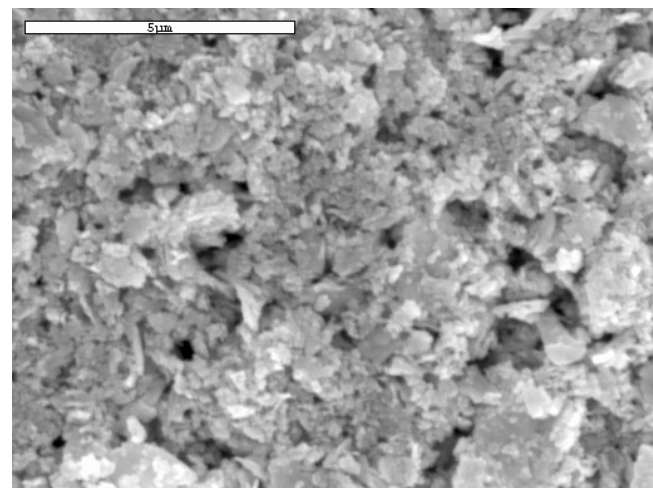

Figure 4.1.3. SEM images of SDC powder by (a) CP and (b) SSS; and GDC powder by (c) CP and (d) Rhodia $^{\circledR}$.

analyzer, of powders synthesized by CP and by SSS was $20-40 \mathrm{~nm}$ and $100-250 \mathrm{~nm}$, respectively. The powders prepared by CP were less dense and had higher surface area than those by SSS, as shown in Table 4.1-1. There was no significant difference in the surface area and SEM morphology of the GDC powders synthesized by CP and from Rhodia ${ }^{\circledR}$. The GDC and SDC powders synthesized by CP yielded high-density bulk samples at lower sintering temperatures because they had superior particle shape, size distribution and surface area, which are all factors influencing powder packing and flow during compaction in the dies.

Table 4.1-1. Specific surface area and pore volume and diameter of powders.

\begin{tabular}{ccccccc}
\hline Materials & \multicolumn{3}{c}{ BET $\left(\mathrm{m}^{2} / \mathrm{g}\right)$} & \multicolumn{2}{c}{ Pore Volume $(\mathrm{cc} / \mathrm{g})$} & \multicolumn{2}{c}{ Pore Diameter $(\AA)$} \\
& $\begin{array}{c}\text { Multipoint } \\
\text { BET }\end{array}$ & $\begin{array}{c}\text { Langmuir } \\
\text { method }\end{array}$ & $\begin{array}{c}\text { BJH } \\
\text { method }\end{array}$ & Total pore volume & $\begin{array}{c}\text { Average } \\
\text { pore dia. }\end{array}$ & $\begin{array}{c}\text { BJH } \\
\text { method }\end{array}$ \\
\hline GDC (CP) & 38.8 & 70.3 & 51.7 & 0.269 & 277.7 & 38.4 \\
GDC (Rhodia $\left.^{\circledR}\right)$ & 24.9 & 45.0 & 30.9 & 0.328 & 524.5 & 19.1 \\
SDC (CP) & 32.7 & 66.0 & 45.5 & 0.550 & 593.1 & 34.6 \\
SDC (SSS) & 2.1 & 3.8 & 2.5 & 0.222 & 41.6 & 28.3 \\
\hline
\end{tabular}




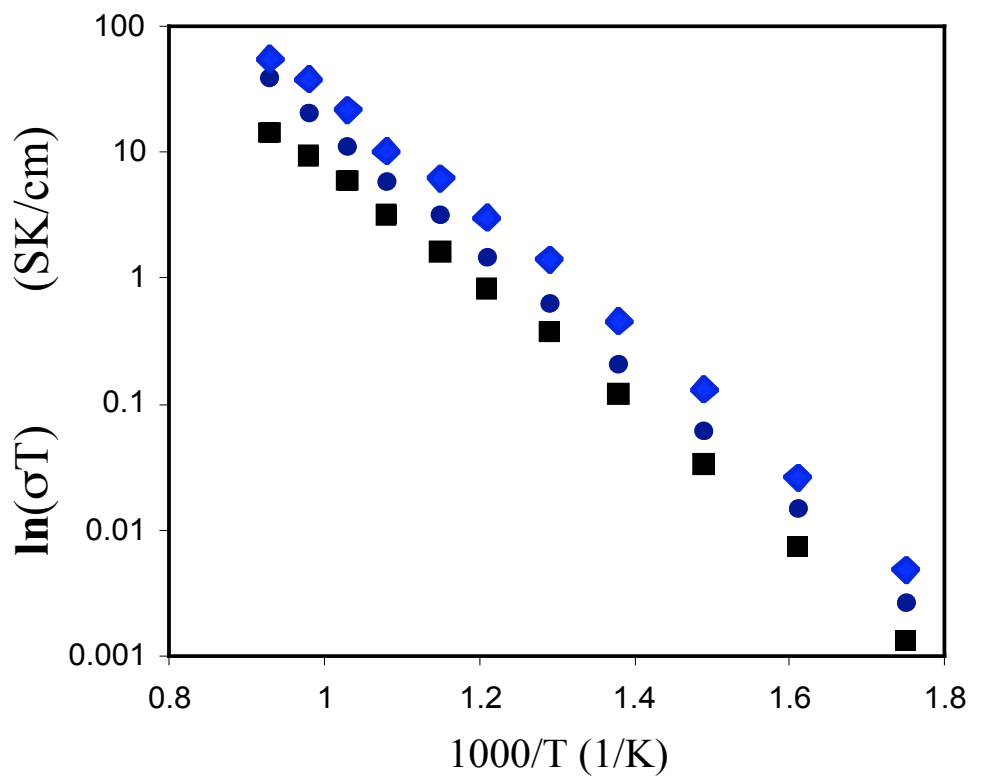

Figure 4.1.4. Total conductivities, in air, of SDC (uniaxial pressing) samples: $(\bullet)$ density, $97.5 \%$, sintering temperature, $1650^{\circ} \mathrm{C},(\diamond) 89.8 \%, 1550^{\circ} \mathrm{C}$, and $(\square) 70 \%, 1450^{\circ} \mathrm{C}$.

\section{Conductivity}

Electrical impedance spectroscopy (EIS) can be used to measure the bulk, grain-boundary, and electrode conduction processes. We used this technique to analyze the grain (or bulk) resistance, $R_{\mathrm{g}}$, and capacitance, $C_{\mathrm{g}}$, and the grain-boundary resistance, $R_{\mathrm{gb}}$, and capacitance, $C_{\mathrm{gb}}$, for various temperatures and microstructures. By combining the resistance and capacitance values with appropriate geometric information from the sample, the corresponding conductivities, $\sigma_{\mathrm{g}}$ and $\sigma_{\mathrm{gb}}$ were derived. This is neither a trivial nor an unambiguous operation, however, and there are currently two prevailing schools of thought that have produced two distinct methodologies, namely the geometric method and the capacitance method [8 - 12].

Effect of sintering temperature and density. The ionic conductivity of SDC electrolytes sintered under various conditions was investigated and correlated with the resulting microstructure. Arrhenius plots of the ionic conductivities measured in air are shown in Fig. 4.1.4. The slopes of the Arrhenius plots - obtained by plotting the following equation:

$$
\ln \sigma T=\ln \sigma^{\circ}-E_{A} k_{B}^{-1} T^{-1}
$$

— give the activation energy, $E_{A}$, for transport of the oxygen ion. In Eq. (4.1.1), $\sigma^{\circ}$ is a pre-exponential factor, $k_{B}$ is Boltzmann's constant and $T$ is temperature 


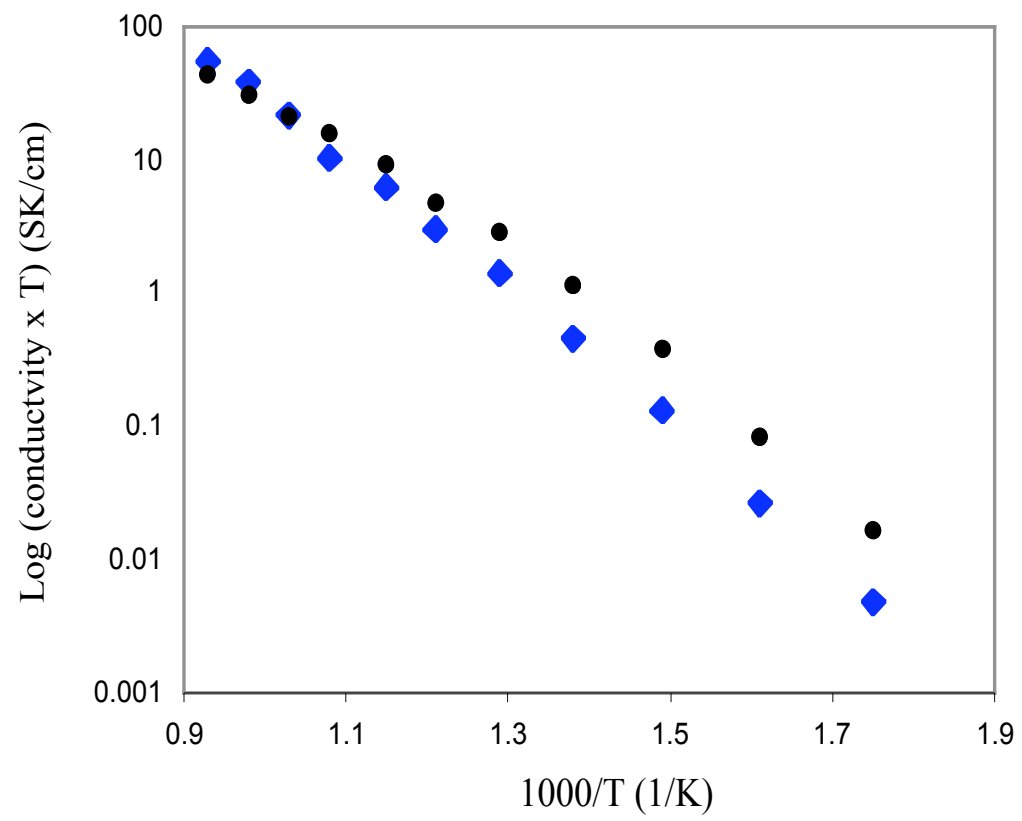

Figure 4.1.5. $\sigma_{\text {tot }}$ for SDC sintered at $1550{ }^{\circ} \mathrm{C}$ for $15 \mathrm{hrs:}(\bullet) \mathrm{CIP}$ and $(\bullet)$ uniaxial pressing.

Figure 4.1.4 shows that sintering conditions and density can change the conductivity of the pellets by as much as an order of magnitude. In general, higher densities result in higher total conductivities. However, the conductivity of $90 \%$ dense SDC (sintered at $1550{ }^{\circ} \mathrm{C}$ ) is higher than that of $98 \%$ dense $\mathrm{SDC}$ (sintered at $1650{ }^{\circ} \mathrm{C}$ ) in the temperature range $300{ }^{\circ} \mathrm{C}-800{ }^{\circ} \mathrm{C}$. That is, the conductivity increased with sintering temperature and time for the samples sintered below $\sim 1550{ }^{\circ} \mathrm{C}$ and decreased at higher sintering temperatures. These trends are discussed in ensuing sections.

Effect of cold-isostatic pressing. The effect of CIP on SDC samples can be observed by the change in $E_{A}$ at $650{ }^{\circ} \mathrm{C}$ in Fig. 4.1.5. Figure 4.1.6 shows that this change was brought about by reduced grain- boundary resistance, for $T<650{ }^{\circ} \mathrm{C}$. Cold-isostatic pressing promotes grain growth which in turn reduces the grain-boundary area (and therefore grain-boundary volume fraction, $V_{g b}$ ). Consequently, the contribution of the grain-boundary to the total conductivity, $\sigma_{t o t}$, decreases. The total conductivity ${ }^{2}$ is the sum of the grain (bulk) conductivity, $\sigma_{g}$, and grain-boundary conductivity, $\sigma_{g b}$, as shown below:

$$
\sigma_{t o t}=\sigma_{g} V_{g}+\sigma_{g b} V_{g b}
$$

where $V_{g}$ is the grain (bulk) volume fraction. It is evident in Eq. (4.1.2) that if $\sigma_{g}>\sigma_{g b}$ (true for SDC) then $\sigma_{t o t}$ increases as $V_{g b}$ decreases. Conversely, above $650{ }^{\circ} \mathrm{C}$, Fig. 4.1 .5 , the effect of a smaller $V_{g b}$

${ }^{2}$ It is assumed that $\sigma_{g}>\sigma_{g b}$ and therefore parallel contributions to the total conductivity may be ignored. 


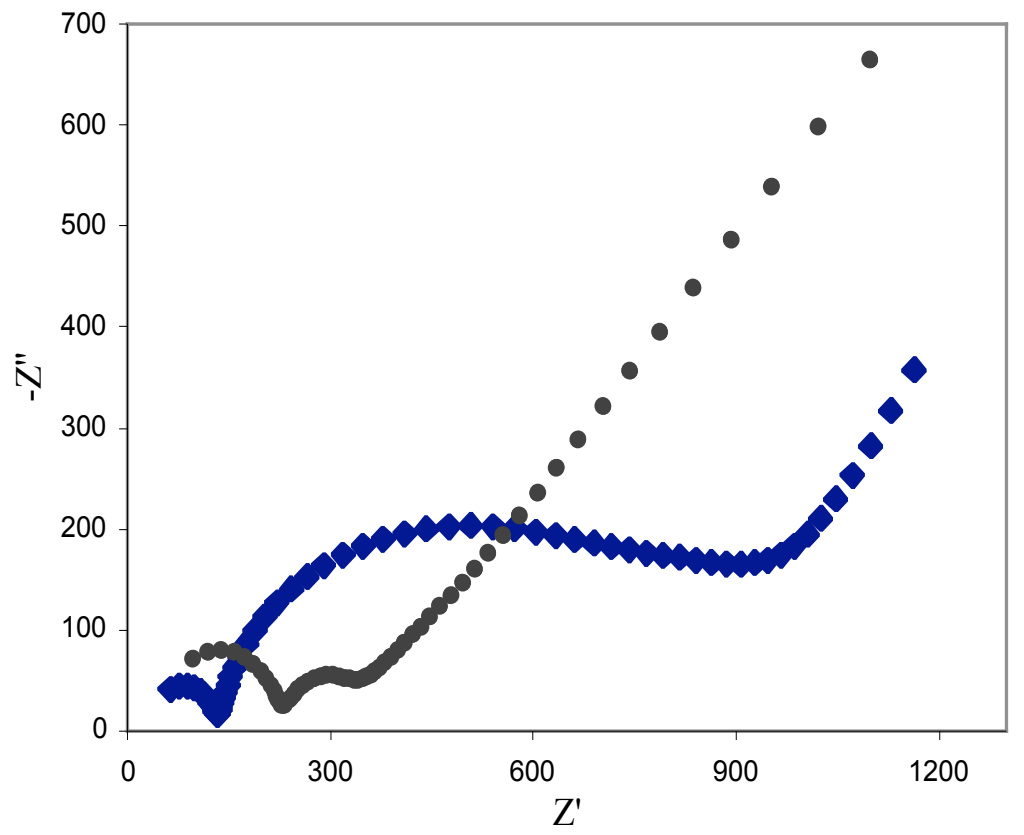

Figure 4.1.6. Impedance plots for SDC samples at $300^{\circ} \mathrm{C} ;(\bullet) \mathrm{CIP}$ and $(\diamond)$ uniaxial pressing.

due to CIP is reduced significantly because differences between the values of $E_{A}$ and $\sigma^{0}$ for $\sigma_{g}$ (lower $\left.E_{A}\right)$ and $\sigma_{g b}\left(\right.$ higher $\left.E_{A}\right)$ dominate at higher temperatures.

Effect of Grain-Boundary Impurities. For ceramics, conductivity depends primarily on a variety of microstructural parameters, e.g. grain size and porosity. In particular, grain boundaries have an important effect on the overall conductivity of most ceramic materials and $\sigma_{\mathrm{gb}}$ is affected by factors such as grain size (discussed earlier) and impurity level, among others. Impurities, including second phases, introduced by the starting materials or during the fabrication procedure have a deleterious effect on $\sigma_{\mathrm{gb}}$ for SDC [8] because they tend to accumulate at grain boundaries during sintering. Various studies $[8,13,14]$ have shown that continuous siliceous phases suppress grain growth resulting in small grain-sizes and larger grain-boundary volume fraction (smaller grain volume fraction). Moreover, these impurities provide a blocking layer within the grain-boundary system that makes the grain-boundary region less conductive. Together these effects significantly reduce the total ionic conductivity of ceria.

Table 4.1-2. Mean Grain Size by Different Sintering Temperatures.

\begin{tabular}{ccc}
\hline Sintering temperature $\left({ }^{\circ} \mathrm{C}\right)$ & Sintering time $($ hrs. $)$ & Mean grain size $(\mu \mathrm{m})$ \\
\hline 1465 & 15 & 1.84 \\
1550 & 15 & 4.21 \\
1650 & 15 & 15.3 \\
\hline
\end{tabular}


Grain-size distributions were estimated using a linear intercept method. They were found to vary with sintering temperature in the range $\sim 2$ to $\sim 15 \mu \mathrm{m}$ as shown in Table 4.1-2. Grain-boundary thicknesses were procured from Christie and Berkel [15] whose data was obtained by high-resolution electron microscopy (HREM). It is observed in Table 4.1-2 that as the sintering temperature increases so does the grain-size. Since the cations are not mobile until high temperature more than $1500{ }^{\circ} \mathrm{C}$, extensive grain growth occurs above $1500{ }^{\circ} \mathrm{C}$. In addition, high sintering temperatures are needed to ensure a homogeneous distribution of dopants in the sample. This concurs with the notion that cation transport is the limiting step for grain growth [16]. However, low cation mobility in the fluorite lattice means that cation segregation - formed by temperature gradients - may arise at elevated temperatures. Accordingly, it was found that changes in the sintering temperature and time can produce variations in both and bulk and grain-boundary conductivity by more than an order of magnitude. That is, sintering conditions have a significant effect on SDC conductivity.

Activation energies and pre-exponential factors for SDC samples are listed in Table 4.1-3. The activation energy and pre-exponential factors for SDC obtained in this work are in good agreement

Table 4.1-3. Activation energies, $E_{A}$, and pre-exponential factors, $\sigma^{0}$, of various SDC samples.

\begin{tabular}{|c|c|c|c|c|c|c|}
\hline Sample thickness $(\mathrm{cm})$ & $\rho_{\text {relative }}(\%)$ & CIP & $\mathrm{T}_{\text {sinter }}\left({ }^{\circ} \mathrm{C}\right)$ & Conductivity Type & $E_{A}(\mathrm{eV})$ & $\sigma_{0}(\mathrm{SK} / \mathrm{cm})$ \\
\hline \multirow[t]{4}{*}{0.172} & 97.5 & No & 1650 & Total & $0.99(3)$ & $2.0 \times 10^{5}$ \\
\hline & & & & Bulk & $0.85(1)$ & $2.0 \times 10^{6}$ \\
\hline & & & & Grain $(\mathrm{G})^{1}$ & $1.0(11)$ & $2.5 \times 10^{4}$ \\
\hline & & & & Grain $(\mathrm{C})^{2}$ & $1.7(01)$ & $4.0 \times 10^{8}$ \\
\hline \multirow[t]{4}{*}{0.183} & 89.5 & No & 1550 & Total & $0.97(5)$ & $3.0 \times 10^{6}$ \\
\hline & & & & Bulk & $0.86(9)$ & $3.0 \times 10^{6}$ \\
\hline & & & & Grain $(G)$ & $1.0(54)$ & $1.8 \times 10^{4}$ \\
\hline & & & & Grain (C) & $1.8(65)$ & $1.0 \times 10^{11}$ \\
\hline \multirow[t]{4}{*}{0.145} & 70.0 & No & 1465 & Total & $0.97(7)$ & $6.8 \times 10^{5}$ \\
\hline & & & & Bulk & $0.75(8)$ & $1.3 \times 10^{5}$ \\
\hline & & & & Grain $(G)$ & $1.0(57)$ & $7.7 \times 10^{4}$ \\
\hline & & & & Grain (C) & $1.8(65)$ & $1.0 \times 10^{11}$ \\
\hline \multirow[t]{5}{*}{0.171} & 90.4 & Yes & 1550 & Total $^{3}$ & $0.97(0)$ & $6.0 \times 10^{6}$ \\
\hline & & & & Total $^{4}$ & $0.66(5)$ & $2.0 \times 10^{7}$ \\
\hline & & & & Bulk & $0.77(7)$ & $3.8 \times 10^{5}$ \\
\hline & & & & Grain $(G)$ & $0.98(4)$ & $2.5 \times 10^{4}$ \\
\hline & & & & Grain $(\mathrm{C})$ & $1.7(53)$ & $1.0 \times 10^{11}$ \\
\hline
\end{tabular}

1. Grain (G): grain-boundary conductivity obtained by using geometrical method.

2. Grain $(\mathrm{C})$ : grain-boundary conductivity obtained by using grain and grain-boundary capacitance

3. Total: total conductivity in temperature range between at $300^{\circ} \mathrm{C}$ and $500{ }^{\circ} \mathrm{C}$.

4. Total: total conductivity in temperature range between at $500{ }^{\circ} \mathrm{C}$ and $800{ }^{\circ} \mathrm{C}$. 
with Tian and Chan [11], and Wang et al.'s results [17]. An inspection of Table 4.1-3 shows that activation energies and pre-exponential factors obtained by the capacitance method [8 - 12] were larger than those obtained by the geometric method [8 - 12]. A similar difference between the two methods was observed by Tian and Chan [11]. The difference between the values of the activation energy and pre-exponential terms, obtained from the capacitance and geometric methods, most likely arises from the inaccuracy in the assumed values of grain size and grain-boundary thickness used in the geometric method [8 - 12]. 


\subsection{Synthesis, Characterization and Optimization of Bismuth Oxide}

In this section, we report our research on bismuth oxide, which consists of three phases. The first phase of our research concerned optimizing the fabrication of bismuth oxide in order to increase its relative density. Accordingly, we explored the benefits of traditional solid-state synthesis versus the increasingly popular wet-chemical methods. The second phase consisted of developing a correct and consistent theory for the structure property relationship in stabilized bismuth oxide. Neutron diffraction and TEM studies enabled us to properly describe the structure of bismuth oxide in the ordered state and the role of dopants in the ordering of the oxygen sublattice below a critical temperature. The insight gained in this endeavor led to the third phase of the research: the development of a new stabilized bismuth oxide that has the highest known oxide ion conductivity among solid oxides. Finally, this knowledge is extended to fluorite-structured oxides in general.

\subsubsection{Fabrication}

\section{Solid-State Synthesis (SSS)}

$\mathrm{Bi}_{2} \mathrm{O}_{3}\left(99.9995 \%\right.$ pure) and $\mathrm{Er}_{2} \mathrm{O}_{3}$ (99.99\% pure) powders were obtained from Alfa Aesar. Stoichiometric amounts of the powders were weighed out, mixed and ball-milled with magnesiastabilized zirconia media in a Nalgene ${ }^{\circledR}$ jar for 20 hours. After decanting and drying, the resulting powder was calcined at $800{ }^{\circ} \mathrm{C}$ for $10 \mathrm{hr}$, and uniaxially pressed at $1450 \mathrm{psi}$ and sintered in air at 890 ${ }^{\circ} \mathrm{C}$ for $15 \mathrm{hrs}$. The resulting ESB pellets were more than $94 \%$ dense. All specimens were polished with 240 to 1200-grit sandpaper (LECO) to acquire even surfaces and desired thickness.

\section{Citrate Process $(\mathrm{CP})$}

ESB powders were prepared by using stoichiometric amounts of $\mathrm{Bi}\left(\mathrm{NO}_{3}\right)_{3} \cdot \mathrm{XH}_{2} \mathrm{O}$ and $\mathrm{Er}\left(\mathrm{NO}_{3}\right)_{3} \cdot 5 \mathrm{H}_{2} \mathrm{O}$. The reactants were dissolved in de-ionized (DI) water. Then ethylene glycol and citric acid were added as the solvent and the metal chelating agent, respectively. The solution was slowly heated on a hot plate and carefully monitored until the DI water evaporated. At this point, the thermo-chemical reaction between nitrate salts occurred, producing a precursor foam. The postthermo-chemical reaction product in this case was a white-yellow ash. The product was calcined at $700{ }^{\circ} \mathrm{C}$ for $2 \mathrm{hrs}$. The crystalline character of the calcined powder was characterized by XRD. For microstructural analysis, the powders were examined using a JSM-35CF scanning electron 


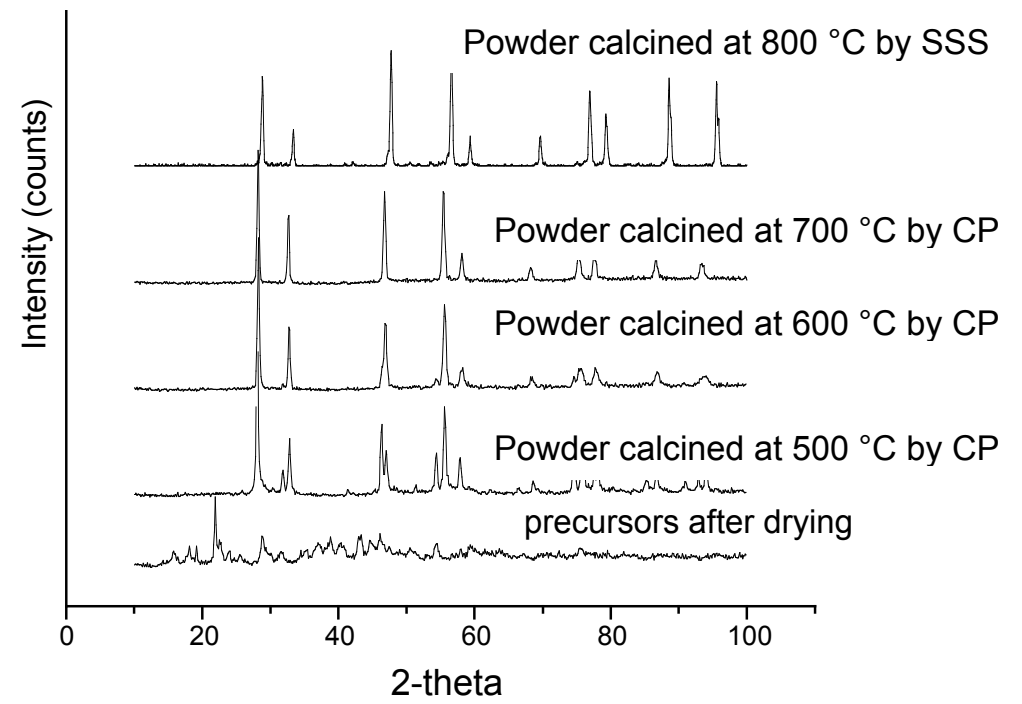

Figure 4.2.1. Structural development to fluorite structure by X-ray diffraction patterns of ESB precursors as increasing calcining temperature

microscope - which was also used to obtain performed energy dispersive analysis (EDS) - a BET (Quantachrome Corporation), and a LS Particle Size Analyzer (Beckman-Coulter).

\subsubsection{Characterization}

Figure 4.2.1 shows that the structure development of the ESB precursors by XRD. As the calcining temperature increases, the fluorite structure is identified more clearly, and finally formed at $700{ }^{\circ} \mathrm{C}$. There are no second phase, such as nitrates and carbonates, present. Figure 4.2.2 shows representative XRD patterns of powders synthesized by both conventional solid-state technique (SSS) and citrate process $(\mathrm{CP})$. Conventionally synthesized powders by SSS are identical to those of

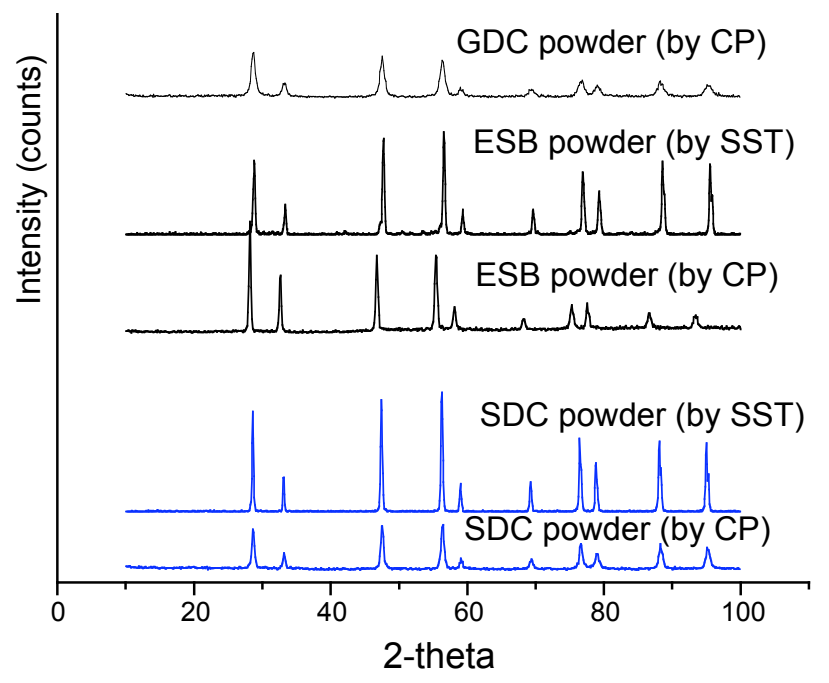

Figure 4.2.2. XRD patterns of calcined SDC, GDC, and ESB powder fabricated by SSS and CP 
(a)

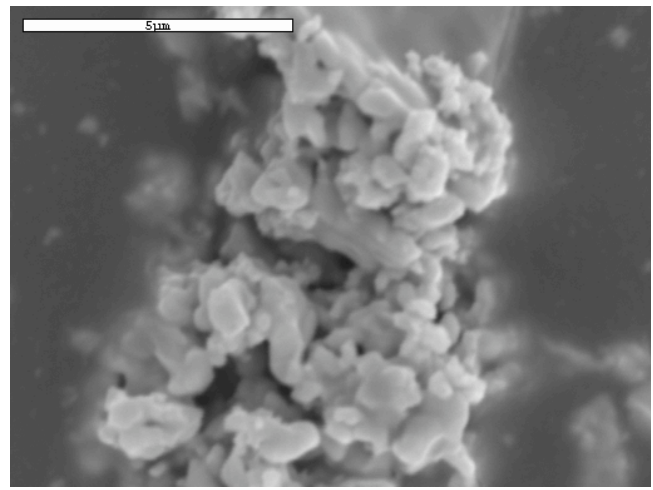

(b)

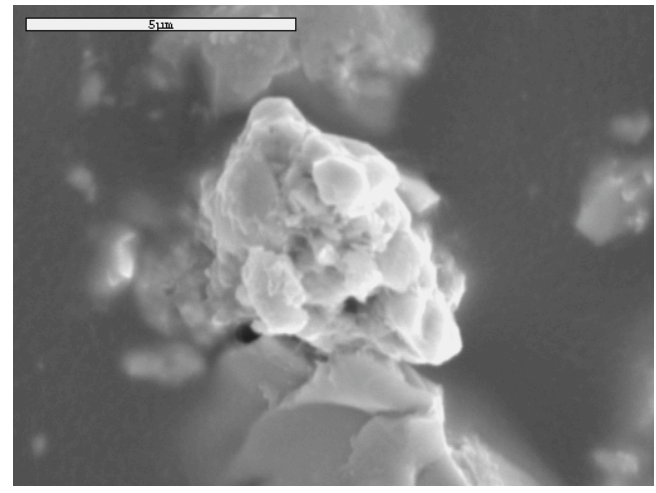

Figure 4.2.3. SEM micrographs of ESB powder fabricated by (a) CP and (b) SSS.

powders by CP, and confirm the formation of cubic SDC, GDC, and ESB. SEM micrographs in Fig. 4.2.3 show the powders synthesized by CP have more faceted polyhedral morphologies, whereas particles by SSS exhibited elliptical morphologies. The average particle size, measured by TEM micrographs and particle size analyzer, of powders synthesized by CP and by SSS was $20-40 \mathrm{~nm}$ and $100-250 \mathrm{~nm}$, respectively. The powders prepared by CP were less dense and had higher surface area than those fabricated by SSS, as shown in Table 4.1-1.

\subsubsection{Neutron Diffraction Study of Occupancy and Positional Order of Oxygen Ions}

$\delta-\mathrm{Bi}_{2} \mathrm{O}_{3}$, the high temperature cubic polymorph of bismuth oxide has the highest ionic conductivity among all the studied oxygen ion conductors [18]. This exceptional conductivity is due to the unique intrinsic defect fluorite structure of this material. The stoichiometry of the material requires 25 percent of the oxygen sublattice to be vacant. Therefore, there is a random distribution of the oxygen ions over an excess number of equipotential sites, resulting in the mobility of all oxygen ions. The high temperature cubic phase undergoes a phase transformation to a poorly conducting monoclinic phase at $723^{\circ} \mathrm{C}$. However, it can be stabilized by doping with several Lanthanide elements [19].

There have been numerous studies on Lanthanide-doped bismuth oxides and these studies have been reviewed thoroughly $[20,21]$. Among the structural models proposed for $\delta-\mathrm{Bi}_{2} \mathrm{O}_{3}$ were those of Sillen [22], Gattow [23] and Willis [24].

In the Gattow model, each of the regular tetrahedral $8 \mathrm{c}$ sites has an equal probability of occupancy by oxygen ions. That is, the oxygen sites are all statistically occupied by 3/4 oxygen ion. This model implies that the oxygen sublattice is completely disordered with respect to occupancy of the $8 \mathrm{c}$ sites. This is consistent with the exceptionally high ionic conductivity, which is generally 
attributed to the defective nature of the oxygen sub-lattice. However, this model fails to explain the presence of a significant fraction of oxygen ions in the interstitial positions of the oxygen sublattice $[25,26]$. Therefore, this model of complete disorder does not accurately describe the structure.

In the Sillen model, 3/4 of the regular tetrahedral sites in the lattice are occupied by oxygen ions and $1 / 4$ of the sites are vacant with the oxygen vacancies ordered in the $<111>$ directions. This model implies that the oxygen sublattice is ordered. However, in the x-ray and electron diffraction experiments of the high temperature structure, no long range $<111>$ ordering of vacancies has been observed. An ordered structure is also inconsistent with the high ionic conductivity of the material above the order-disorder transition temperature.

In the Willis model the oxygen ions are displaced along four of the $<111>$ directions from the regular tetrahedral sites towards the central octahedral vacant site. This gives occupancy of $3 / 16$ at each of the 32 equivalent positions ( $32 \mathrm{f}$ sites) in the unit cell. This model describes the positional disorder in the oxygen sublattice. However, according to this model, there is no occupancy of oxygen ions in the regular tetrahedral sites, which is inconsistent with the neutron diffraction studies $[25,26]$ of this material that show occupancy of oxygen ions in both $8 \mathrm{c}$ and $32 \mathrm{f}$ sites.

All three models are inconsistent with the observed experimental results and consequently, they need to be modified to explain the complex structural changes and their effect on the transport phenomena in the mobile sublattice and ultimately, the conductivity of the material. The neutron diffraction studies of Battle et al $[25,26]$ and Verkerk et al $[27,28]$ performed for the disordered anion sublattice show that the anion sublattice is a combination of the Gattow model (3/4 occupancy at 8c positions) and the Willis model (average occupancy of $32 \mathrm{f}$ positions). These studies are consistent with the high ionic conductivity of this material, which is attributed to the disordered nature of the oxygen sublattice above the order-disorder transition temperature. Their studies did not indicate any type of $<111>$ Sillen type of ordering in the disordered structures.

The phase-stabilized bismuth oxides undergo an order-disorder transition of the oxygen sublattice at about $600^{\circ} \mathrm{C}$ resulting in a change in conductivity activation energy [27, 28]. It has been reported by Wachsman et al that the conductivity of phase-stabilized bismuth oxides decays when annealed at temperatures less than the transition temperature. This degradation in conductivity with time was attributed to ordering of the mobile oxygen sublattice [29, 30]. There has been some controversy regarding the theory of anion ordering and the aging phenomenon has been attributed to cation ordering by Fung et al [31]. The structural modeling of the oxygen sublattice by Wachsman et 
al based on TEM and neutron diffraction has shown the decay in conductivity to be caused by the

formation of a $<111>$ alignment of oxygen vacancies as well as Willis type $<111>$ displacements of oxygen ions $[32,33]$. The oxygen ion ordering mechanism was also confirmed by studying the dependence of kinetics of the aging phenomenon on dopant type and dopant concentration [34].

Neutron Diffraction studies were performed to investigate the effect of doping on the structural changes in the oxygen sublattice resulting in the conductivity decay. The oxygen sublattice of both ordered and disordered structures of bismuth oxide, doped with Yb, Er, Y, Ho, and Dy, were studied. The change in occupancy of oxygen ions in the $8 \mathrm{c}$ and $32 \mathrm{f}$ positions was determined for each dopant.

\section{Experimental}

Polycrystalline samples of $\mathrm{M}_{2} \mathrm{O}_{3}-\mathrm{Bi}_{2} \mathrm{O}_{3}(\mathrm{M}=\mathrm{Yb}$, Er, Y, Ho, Dy) were prepared by a solid-state reaction from a stoichiometric mixture of $\mathrm{Bi}_{2} \mathrm{O}_{3}\left(99.999 \%\right.$ pure) and $\mathrm{M}_{2} \mathrm{O}_{3}$ (99.99\% pure) powders. The powders of required composition $\left(25 \% \mathrm{M}_{2} \mathrm{O}_{3}\right)$ were mixed and ground. The mixed powders were calcined at $800{ }^{\circ} \mathrm{C}$ for $16 \mathrm{hr}$, and then ground again and pressed into pellets of $2.5 \mathrm{~cm}$ diameter, which were subsequently sintered in air at $900{ }^{\circ} \mathrm{C}$ for $16 \mathrm{hr}$. Some of the pellets were then aged in air at 500 ${ }^{\circ} \mathrm{C}$ for $300 \mathrm{hr}$.

Neutron diffraction studies were performed at the High Flux Isotope Reactor facility at Oak Ridge National Laboratory using the high-resolution neutron powder diffractometer. The monochromator is a (115) Ge crystal, which can be oriented to select incident wavelengths of 1.0, 1.4, 2.2, and 4.2A. Powdered samples were held in $1 \mathrm{~cm}$ diameter vanadium cans with ceramic lids. The data was collected at room temperature for the aged and the unaged samples of $\mathrm{M}_{2} \mathrm{O}_{3}-\mathrm{Bi}_{2} \mathrm{O}_{3}(\mathrm{M}=\mathrm{Yb}$, $\mathrm{Er}, \mathrm{Y}, \mathrm{Ho}, \mathrm{Dy})$. The data was also collected for $\mathrm{Yb}_{2} \mathrm{O}_{3}-\mathrm{Bi}_{2} \mathrm{O}_{3}$ at $11 \mathrm{~K}, 75 \mathrm{~K}, 150 \mathrm{~K}$, and $225 \mathrm{~K}$ by placing the powdered sample in a Helium refrigerator. The structural parameters were refined using the software General Structural and Analysis System (GSAS) developed by A. Larson and R. Von Dreele at Los Alamos National Laboratory [36].

\section{Results and Discussion}

The neutron diffraction data was refined using the least squares Rietveld refinement procedure using the software GSAS. During the refinement process, the fraction of oxygen ions at three different sites $(8 \mathrm{c}, 32 \mathrm{f}$, and $48 \mathrm{i})$ were used as variables. In addition, the $\mathrm{x}$ values of $32 \mathrm{f}(\mathrm{x}, \mathrm{x}, \mathrm{x})$ and $48 \mathrm{i}(0.5, \mathrm{x}$, 
$\mathrm{x}$ ) sites were also allowed to vary. As a starting point, we refined the diffraction data of both aged and unaged samples with all the dopants assuming Sillen, Gattow, and Willis models.

The residuals $\left(\chi^{2}\right.$ and $\left.R_{w p}\right)$ for the Sillen, Gattow, and Willis models are shown in Table 4.2-1(a), (b) and (c), respectively, for each of the samples. A good fit with the diffraction patterns is indicated by low values of residuals $\left(\mathrm{R}_{\mathrm{wp}} \sim 0\right.$ and $\left.\chi^{2} \sim 1\right)$. From the tables, it is clear that none of the models by themselves is consistent with the observed results for either the ordered or the disordered cases. The residuals are high for the Gattow model both for ordered and disordered data sets. They are relatively low with the Sillen model with the ordered data set. In general, a good fit is not obtained for either of these models, by themselves. However, the Sillen model is a better fit for the ordered structure and the Gatow model is a better fit for the disordered structure. In addition, the deviations are significantly lower in the case of the Willis model for ordered structures. This indicates the importance of displacements during the ordering process. Nevertheless, the residuals obtained are large enough to merit further refinement by modifying the above models.

The TEM diffraction patterns of the ordered structures give additional weak reflections that correspond to an ordered structure consisting of $<111>$ vacancy ordering $[30,32,33]$. Therefore, we must take the occupancy of regular 8c sites ("occupancy ordering"), corresponding to the Sillen model into consideration for the ordered structures. The previous neutron diffraction study on $\left(\mathrm{Bi}_{2} \mathrm{O}_{3}\right)_{0.80}\left(\mathrm{Er}_{2} \mathrm{O}_{3}\right)_{0.20}$ has shown that there is an extensive displacement of oxygen ions from the regular $8 \mathrm{c}$ positions to the $32 \mathrm{f}$ interstitial positions ("positional ordering") corresponding to the Willis model [37]. This study also indicates that the fraction of oxygen ions in the 48i sites does not change significantly with aging. Therefore, we must take into consideration the combination of Sillen (occupancy ordering) and Willis (positional ordering) models for the ordered structures.

In the case of disordered structures, there have been neutron diffraction studies [22, 23] that show no occupancy ordering. However, these studies also show that there is a significant fraction of oxygen ions in the $32 \mathrm{f}$ positions (positional ordering). Therefore, for the refinement of disordered structures, we start with a combination of random occupancy and positional ordering, i.e, the combination of Gattow and Willis models respectively.

The oxygen ions are distributed among three sites- $8 \mathrm{c}, 32 \mathrm{f}$ and $48 \mathrm{i}$ in the fluorite unit cell. Varying the fraction of oxygen ions in the three sites simultaneously led to either a divergence of equations, or a convergence with high residuals and the total sum of oxygen ions greater than 6 . In order to achieve stable convergence, we constrained the fraction of oxygen ions to vary as two distinct 
pairs. One pair consisted of oxygen ions occupying 8c sites and half of $48 \mathrm{i}$ sites and the other pair comprised of oxygen ions in $32 \mathrm{f}$ sites and the other half of $48 \mathrm{i}$ sites. In the first pair, the oxygen ions in $48 \mathrm{i}$ sites were constrained to decrease by $8 / 48$ when there is an increase in 8c occupancy by 1 . Similarly, the oxygen ions occupying the $48 \mathrm{i}$ sites in the second pair were constrained to decrease by a factor of $32 / 48$ when there is an increase in $32 \mathrm{f}$ occupancy by 1 . By using the pairs of oxygen ion occupancies, the effective sum of oxygen ions in the unit cell could be maintained at 6 .

Table 4.2-1. Residuals for Rietveld refinement based on (a) Sillen model, (b) Gattow model and (c) Willis model

\begin{tabular}{|c|c|c|c|}
\hline & Structure & Rwp & $x^{2}$ \\
\hline \multicolumn{4}{|l|}{ (a) } \\
\hline \multirow[t]{2}{*}{ YbSB } & Disordered & 0.47 & 176 \\
\hline & Ordered & 0.23 & 75 \\
\hline \multirow[t]{2}{*}{ ESB } & Disordered & 0.43 & 173 \\
\hline & Ordered & 0.26 & 83 \\
\hline \multirow[t]{2}{*}{ YSB } & Disordered & 0.48 & 183 \\
\hline & Ordered & 0.25 & 81 \\
\hline \multirow[t]{2}{*}{ HoSB } & Disordered & 0.52 & 189 \\
\hline & Ordered & 0.23 & 84 \\
\hline \multirow[t]{2}{*}{ DySB } & Disordered & 0.49 & 166 \\
\hline & Ordered & 0.24 & 96 \\
\hline \multicolumn{4}{|l|}{ (b) } \\
\hline \multirow[t]{2}{*}{ YbSB } & Disordered & 0.38 & 123 \\
\hline & Ordered & 0.52 & 181 \\
\hline \multirow[t]{2}{*}{ ESB } & Disordered & 0.36 & 143 \\
\hline & Ordered & 0.53 & 193 \\
\hline \multirow[t]{2}{*}{ YSB } & Disordered & 0.33 & 154 \\
\hline & Ordered & 0.52 & 185 \\
\hline \multirow[t]{2}{*}{ HoSB } & Disordered & 0.42 & 135 \\
\hline & Ordered & 0.48 & 180 \\
\hline \multirow[t]{2}{*}{ DySB } & Disordered & 0.31 & 123 \\
\hline & Ordered & 0.50 & 189 \\
\hline \multicolumn{4}{|l|}{ (c) } \\
\hline \multirow[t]{2}{*}{ YbSB } & Disordered & 0.39 & 138 \\
\hline & Ordered & 0.16 & 21 \\
\hline \multirow[t]{2}{*}{ ESB } & Disordered & 0.41 & 151 \\
\hline & Ordered & 0.19 & 24 \\
\hline \multirow[t]{2}{*}{ YSB } & Disordered & 0.34 & 142 \\
\hline & Ordered & 0.17 & 20 \\
\hline \multirow[t]{2}{*}{ HoSB } & Disordered & 0.42 & 162 \\
\hline & Ordered & 0.21 & 50 \\
\hline \multirow[t]{2}{*}{ DySB } & Disordered & 0.36 & 126 \\
\hline & Ordered & 0.29 & 79 \\
\hline
\end{tabular}


The observed and calculated diffraction patterns, as well as the difference between the observed and calculated diffraction patterns, for unaged and aged ESB are shown in Table 4.2-2. Another indication of the quality of the fit is shown by square of the ratio of observed (Fo) to calculated (Fc) structure factors (determined by GSAS) for each of the allowed reflections. Most of the reflections have ratios close to 1 for both aged and unaged samples, indicating a good fit. Some of the allowed reflections in the aged structure ( (111) and (321) ) are not observed (Fo=0). However, their calculated structure factors are also close to zero and it is possible that these reflections are too weak to be detected.

From Table 4.2-2 it is seen that the aged ESB sample gives additional weaker reflections in addition to the strong fluorite reflections present in the diffraction pattern of the unaged sample. These reflections are consistent with the superlattice reflections observed in TEM diffraction patterns [33].

Table 4.2-2. Observed and calculated structure factors for (a) unaged and (b) aged ESB

\begin{tabular}{|c|c|c|c|}
\hline hkl & $|\mathrm{Fo}|^{2} \times 10^{4}$ & $|\mathrm{Fc}|^{2} \times 10^{4}$ & $|\mathrm{Fo}|^{2} /|\mathrm{Fc}|^{2}$ \\
\hline \multicolumn{4}{|l|}{ (a) } \\
\hline 111 & 4.35 & 4.39 & 0.991 \\
\hline 200 & 0.31 & 0.43 & 0.721 \\
\hline 220 & 8.79 & 8.75 & 1.001 \\
\hline 311 & 3.34 & 3.24 & 1.031 \\
\hline 222 & 0.62 & 0.47 & 1.320 \\
\hline 400 & 4.47 & 4.01 & 1.115 \\
\hline 331 & 1.13 & 0.98 & 1.153 \\
\hline \multicolumn{4}{|l|}{ (b) } \\
\hline 110 & 0.00 & 0.08 & - \\
\hline 200 & 0.15 & 0.12 & 1.250 \\
\hline 211 & 0.18 & 0.17 & 1.058 \\
\hline 220 & 0.67 & 0.56 & 1.196 \\
\hline 310 & 0.20 & 0.24 & 0.833 \\
\hline $222^{*}$ & 35.35 & 34.67 & 1.028 \\
\hline 321 & 0.00 & 0.04 & - \\
\hline $400 *$ & 3.08 & 4.23 & 0.728 \\
\hline 330 & 0.48 & 0.39 & 1.231 \\
\hline 411 & 0.14 & 0.11 & 1.273 \\
\hline 420 & 1.03 & 0.85 & 1.212 \\
\hline 332 & 1.45 & 1.26 & 1.150 \\
\hline 422 & 1.97 & 2.12 & 0.929 \\
\hline 431 & 0.75 & 0.61 & 1.229 \\
\hline 510 & 0.26 & 0.19 & 1.368 \\
\hline 521 & 0.36 & 0.27 & 1.333 \\
\hline $440 *$ & 67.56 & 65.25 & 1.035 \\
\hline
\end{tabular}

* Reflections due to the original fluorite structure 
Table 4.2-3. Occupancies of oxygen ions in regular (8c) and interstitial (32f and 48i) sites in (a) unaged and (b) aged structures from models fitted with the observed neutron diffraction data.

\begin{tabular}{cccccc}
\hline Dopant & Occupancy of 8c sites & Occupancy of 32f sites & Occupancy of 48i sites & $\mathrm{R}_{\mathrm{wp}}$ & $\chi^{2}$ \\
\hline (a) & & & & & \\
$\mathrm{Yb}$ & 1.12 & 1.56 & 0.32 & 0.032 & 1.08 \\
$\mathrm{Er}$ & 1.14 & 1.59 & 0.27 & 0.023 & 1.01 \\
$\mathrm{Y}$ & 1.18 & 1.52 & 0.30 & 0.028 & 1.02 \\
$\mathrm{Ho}$ & 1.26 & 1.47 & 0.27 & 0.035 & 1.13 \\
$\mathrm{Dy}$ & 1.35 & 1.39 & 0.26 & 0.057 & 1.19 \\
$\mathrm{(b)}$ & & & & & \\
$\mathrm{Yb}$ & & & 0.34 & 0.057 & 1.48 \\
$\mathrm{Er}$ & 0.05 & 2.61 & 0.29 & 0.083 & 1.53 \\
$\mathrm{Y}$ & 0.06 & 2.65 & 0.30 & 0.042 & 1.44 \\
$\mathrm{Ho}$ & 0.09 & 2.62 & 0.33 & 0.037 & 1.09 \\
$\mathrm{Dy}$ & 0.42 & 2.25 & 0.31 & 0.048 & 1.16 \\
\hline
\end{tabular}

The residual values of refinement were plotted against the occupancy of oxygen ions in 8c positions for both ordered and disordered structures. From the plots, it was shown that the fraction of oxygen ions displaced the $8 \mathrm{c}$ sites during the ordering process is least for Dy doped structure and highest for $\mathrm{Yb}$ and Er doped structures. The oxygen ion occupancies and the residuals in the refined structures of aged and unaged samples are shown in Table 4.2-3. From Table 4.2-3 it is observed that the change in occupancy of oxygen ions in $48 \mathrm{i}$ sites is not significant. The significant change is the displacement of oxygen ions from $8 \mathrm{c}$ to $32 \mathrm{f}$ sites. The time constants for conductivity decay (aging), taken from [34] and the change in structural parameters of oxygen sublattice due to aging, 1 $8 c($ aged)/8c(unaged) are shown in Table 4.2-4 for each dopant.

The change in oxygen ion occupancy due to doping is plotted as a function of time constant for aging in Fig. 4.2.4. The figure shows that the higher the kinetics of conductivity decay, the higher is the displacement of oxygen ions from the tetrahedral $8 \mathrm{c}$ sites to the interstitial $32 \mathrm{f}$ positions. This

Table 4.2-4. Change in oxygen ion occupancy due to aging

\begin{tabular}{ccc}
\hline Dopant & Time Constant for Aging $(\mathrm{h})$ & $\begin{array}{c}\text { Change in occupancy due to aging } \\
1-8 \mathrm{c}(\text { aged }) / 8 \mathrm{c}(\text { unaged) }\end{array}$ \\
\hline $\mathrm{Yb}$ & 4.177 & 0.9550 \\
$\mathrm{Er}$ & 24.823 & 0.9473 \\
$\mathrm{Y}$ & 53.789 & 0.9237 \\
$\mathrm{Ho}$ & 79.412 & 0.6667 \\
$\mathrm{Dy}$ & 170.00 & 0.1185 \\
\hline
\end{tabular}




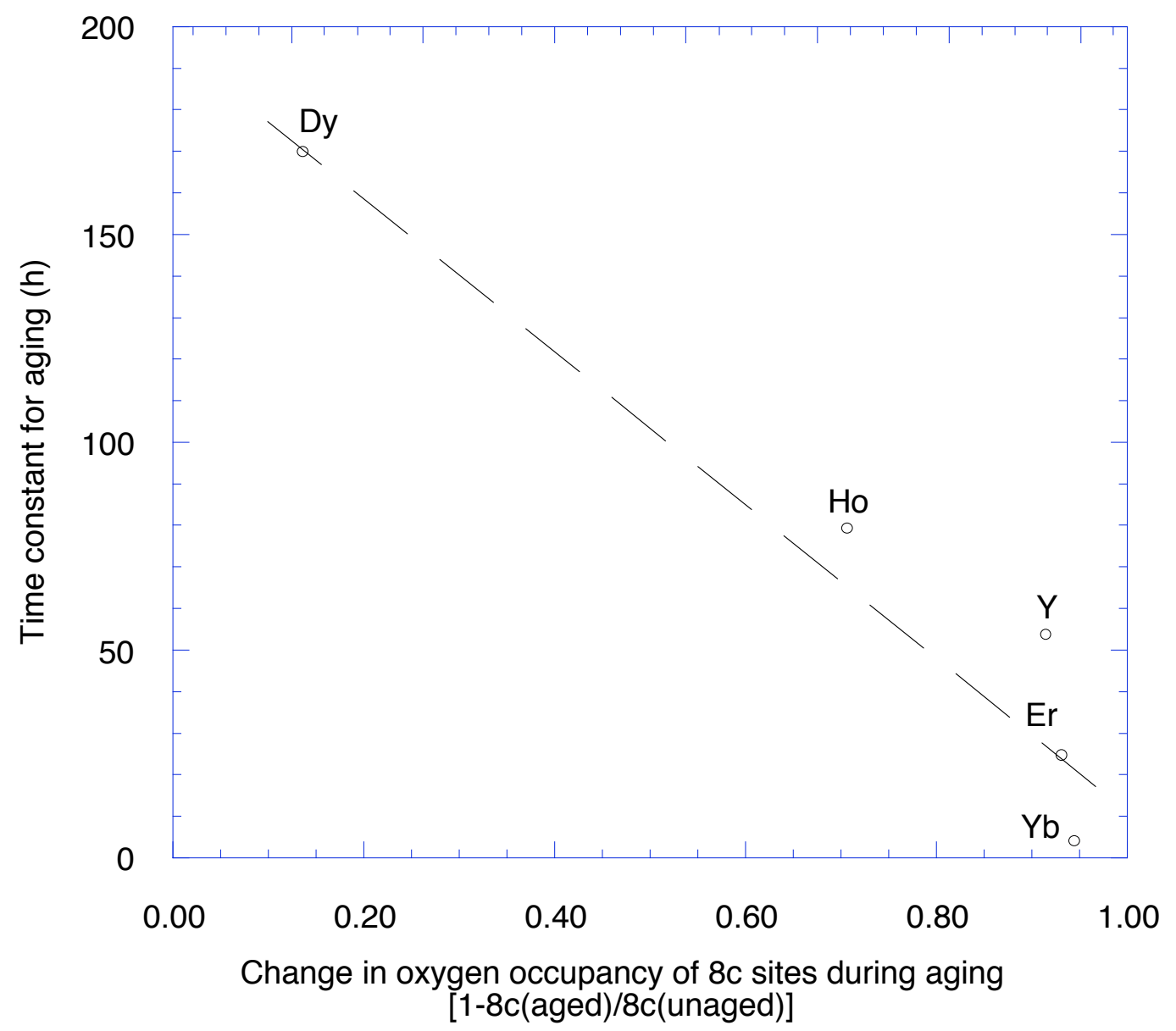

Figure 4.2.4. Correlation between time constant for aging and change in oxygen ion occupancies (line drawn to guide the eye).

clearly demonstrates the correlation between the structural arrangement of the oxygen sublattice and the decay in ionic conductivity; and confirms the anion ordering theory to describe the aging process.

The occupancy and positional order of the anion sublattice obviously affect the oxygen ion transport mechanism. This mechanism therefore needs to include the occupancy of interstitial positions. These points are discussed in detail in [35].

\section{Conclusions}

The decay in oxygen ion conductivity in cubic bismuth oxides stabilized by isovalent Lanthanide cations when annealed below the order-disorder transition temperature is due to ordering of the oxygen sublattice. The $<111>$ vacancy ordering is accompanied by the displacement of oxygen ions from the regular tetrahedral $8 \mathrm{c}$ sites to the interstitial $32 \mathrm{f}$ sites. Thus, the ordered structures cannot be described on the basis of any single existing structural models for this material. The refinements of neutron 
diffraction data show that the disordered structures can be satisfactorily described in terms of a combination of Gattow and Willis models, whereas the ordered structures are consistent with a mixed Sillen - Willis models.

\subsubsection{Effect of Dopant Polarizability on Oxygen Sublattice Order}

At about $600^{\circ} \mathrm{C}$ all of the cubic phase-stabilized bismuth oxides exhibit a change in conductivity activation energy, with a lower activation energy above $\sim 600^{\circ} \mathrm{C}$ and a higher activation energy below $\sim 600^{\circ} \mathrm{C}[38]$. Verkerk and Burgraff [39] attributed this to an order-disorder transition of the oxygen sublattice. Below the order-disorder transition temperature we reported a reversible time-dependent decay in conductivity [38, 40, 41]. We investigated this "aging" phenomenon using thermal analysis and XRD, TEM and neutron diffraction [32, 40 - 46]. The nature of the ordered structure, below the order-disorder transition temperature, cannot be detected by XRD because of weak scattering of x-rays by oxygen ions. It is however detectable by TEM studies which show the formation of a $2 \mathrm{a} \times 2 \mathrm{a} \times 2 \mathrm{a}$ superlattice. The ordered structure is significantly more difficult to ascertain than the basic fluorite structure because of this superlattice. We studied several structural models for the ordered structures and have shown that a $<111>$ vacancy ordering (similar to the occupancy ordering of Sillen) is most consistent with the TEM diffraction studies [44, 45].

Neutron diffraction experiments indicate that the vacancy ordering process is accompanied by the displacement of nearly all the oxygen ions from the $8 \mathrm{c}$ to $32 \mathrm{f}$ positions [43, 46]. While displacement of oxygen ions from $8 \mathrm{c}$ to $32 \mathrm{f}$ sites is the positional disorder described by Willis, it is only "positional disorder" if each of the equivalent $32 \mathrm{f}$ positions has $3 / 16$ occupancy. If, however, there is a concerted or preferential occupancy of the $32 \mathrm{f}$ sites such that there is an occupancy of 1 in a particular $32 \mathrm{f}$ site and all other equivalent sites are empty then this displacement is in fact a "positional order". Rietveld refinement of neutron diffraction data of the ordered structure shows that it is best described by a combination of occupancy and positional ordering [46].

Figure 4.2.5 shows the anion sublattice of the superstructure that resulted in the best fit with the TEM and neutron diffraction data. The structure has the $<111>8 \mathrm{c}$-site vacancy ordering associated with the Sillen model (occupancy ordering). Superimposed on this occupancy ordering is displacement of occupied $8 \mathrm{c}$ sites in specific $<111>$ directions (positional ordering) as indicated by the arrows. The orientation of the $<111>$ occupancy ordering alternates from one adjacent fluorite unit cell to another. This results in additional $<110>$ vacancy ordering between unit cells. $<110>$ vacancy 


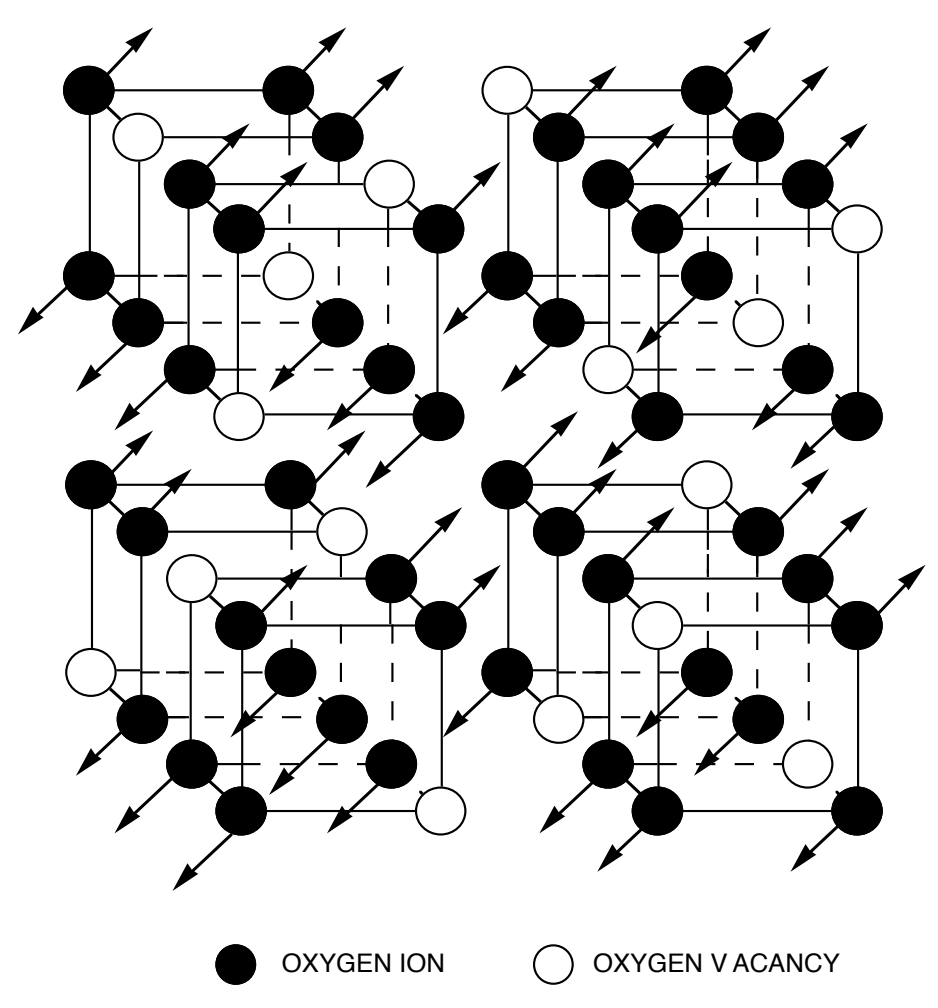

Figure 4.2.5. Model of ordered structure showing both occupancy ordering (filled vs. vacant 8c sites) and positional ordering $(<111>$ displacement toward $32 \mathrm{f}$ sites $)$.

ordering was proposed by Battle et al. as a stable configuration due to the polar nature of the $6 \mathrm{~s}$ electron pair of $\mathrm{Bi}^{3+}[47,48]$.

Therefore, the polarizability of the cations plays an intrinsic role in both the ordered structure of the anion sublattice and the stability of the disordered structure. All of the dopants typically used to stabilize the cubic structure of $\mathrm{Bi}_{2} \mathrm{O}_{3}$ are less polar than $\mathrm{Bi}^{3+}$ and it is the affect of their polarizability that is reported in this section.

\section{Experimental}

As-sintered pellets of $\mathrm{M}_{2} \mathrm{O}_{3}-\mathrm{Bi}_{2} \mathrm{O}_{3}(\mathrm{M}=\mathrm{Yb}, \mathrm{Er}, \mathrm{Y}, \mathrm{Ho}$, Dy) were polished to obtain planar surfaces. Engelhard gold paste was applied to the polished surfaces and annealed at $800{ }^{\circ} \mathrm{C}$ for 4 hours to obtain porous gold electrodes. A one inch long gold wire was attached to each gold electrode using the gold paste, and annealed at $800{ }^{\circ} \mathrm{C}$ for 4 hours. Some of the pellets were then aged in air at $500{ }^{\circ} \mathrm{C}$ to obtain the conductivity decay time constant [41]. Conductivity measurements over a frequency range $20 \mathrm{~Hz}$ to $1 \mathrm{MHz}$ were made using a two-probe AC impedance method with an $\mathrm{HP}$ 4282A 
precision impedance LCR (inductance-capacitance-resistance) meter. The dielectric constant for bulk anion transport at $200-300^{\circ} \mathrm{C}$ was determined from the impedance results.

\section{Results and Discussion}

The aging phenomenon occurs when "phase-stabilized" bismuth oxides are annealed below the order-disorder transition temperature. It is distinct from a conventional crystallographic phase transformation, but results in formation of a superstructure observable by TEM diffraction patterns and ordering of the oxygen sublattice as observed by neutron diffraction. The resulting conductivity decay is temperature-time dependent. As the temperature is decreased below the order-disorder transition temperature the driving force for ordering increases, resulting in an increase in the rate of conductivity decay. However, a continuing decrease in temperature limits the kinetics of ordering resulting in a maximum in the rate of conductivity decay at about $500{ }^{\circ} \mathrm{C}[38,41]$.

The time dependence of the conductivity decay can be represented by an empirical equation:

$$
\sigma(\mathrm{t})=\sigma(\infty)+[\sigma(0)-\sigma(\infty)] \exp \left[-(\mathrm{t} / \tau)^{\beta}\right]
$$

where $\sigma(0)$ is the initial conductivity, $\sigma(\infty)$ is the conductivity at infinite time, $\beta$ is a dimensionless parameter, and $\tau$ is the pertinent time constant [41]. The time constant is an indicator of the rate of decay and therefore the kinetic stability of the disordered vs. the ordered structure, with greater $\tau$ indicating a greater degree of stability of the disordered structure. The time constant at $500{ }^{\circ} \mathrm{C}$ was determined as a function of dopant and dopant concentration [41]. It was found that the $\log (\tau)$ increased linearly with dopant radii. A 4\% increase in dopant radii, from $\mathrm{Yb}^{3+}$ to $\mathrm{Dy}^{3+}$, increased the stability of the disordered structure from $\tau=4 \mathrm{~h}$ to $171 \mathrm{~h}$, respectively, for $25 \%$ doping.

Shirao et al. studied the correlation between the polarizability and the effective ionic radius in lanthanide elements [49]. They arrived at an empirical relationship indicating that the polarizability is

proportional to the cube of ionic radius. Using their relationship, we calculated the polarizabilities of the dopant cations. The correlation between the polarizability of dopant cations and time constant for aging is shown in Fig. 4.2.6. From the figure, it is clear that there is a strong linear dependence between the dopant cation polarizability and the kinetics of aging. This is also consistent with the fact that the conductivity in cubic bismuth oxides is reduced due to substitution of highly polarizable bismuth ions with less polarizable lanthanide ions in the cation sublattice. In their structural analysis, Battle et al. propose that bismuth ions are highly polarizable due to the presence of lone-pair of 


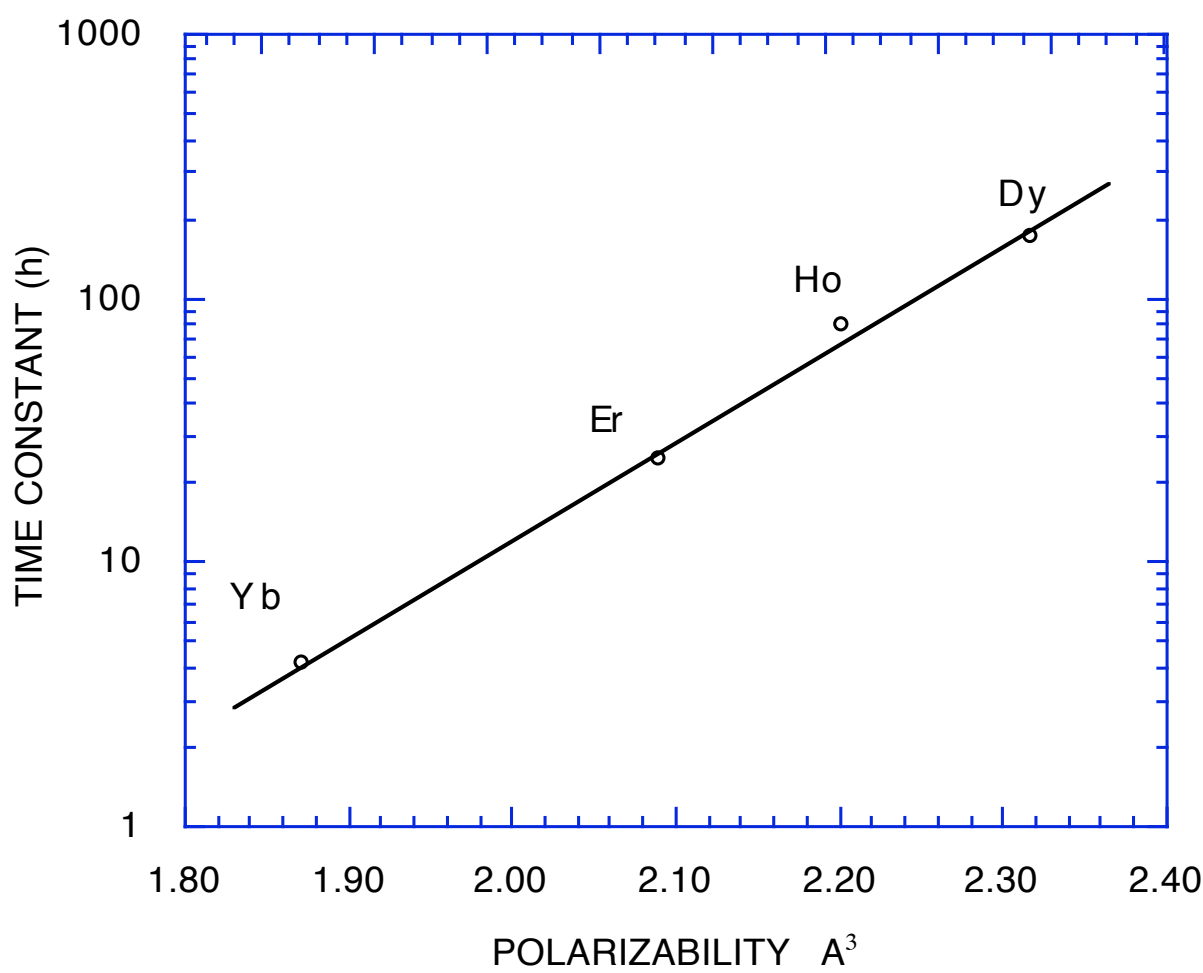

Figure 4.2.6. Relationship between conductivity decay time constant and dopant polarizability.

electrons in their outer shell $[47,48]$. This high polarizability makes it very unlikely for a $<111>$ vacancy arrangement in the oxygen sublattice. They also proposed that a less polarizable ion such as $\mathrm{Y}^{3+}$ would prefer a $<111>$ vacancy arrangement. Our results are clearly consistent with this analysis.

Since there is a relationship between the polarizability and the kinetics of aging, there should also be a relationship with the dielectric constant. Thus, the dielectric constants $(\kappa)$ were calculated from the bulk components of the AC impedance spectra for unaged samples at $200{ }^{\circ} \mathrm{C}, 250{ }^{\circ} \mathrm{C}$, and $300{ }^{\circ} \mathrm{C}$. As shown in Fig. 4.2.7, there is a linear relationship between $\tau$ and $\kappa$ for all of the dopants except Yttrium. This linear relationship confirms the effect of polarizability of the cation sublattice on kinetics of ordering of the oxygen sublattice. The fact that $\mathrm{Y}^{3+}$ does not belong to the same periodic group as the rest of the dopants may explain its deviation from the linear dependence observed with the lanthanide dopants. The polarizability depends upon the shielding of nucleus by the valence electrons. Therefore, the polarizability of ions with $f$ valence electrons (lanthanides) would be different from that of $\mathrm{Y}^{3+}$ ion.

From Figs. 4.2.6 and 4.2.7, it is evident both empirically and experimentally that there is a strong correlation between the dopant polarizability and the kinetics of the ordering process. It was argued by Fung et al. that due to the dependence of the kinetics of aging on dopant ionic radii, the conductivity 


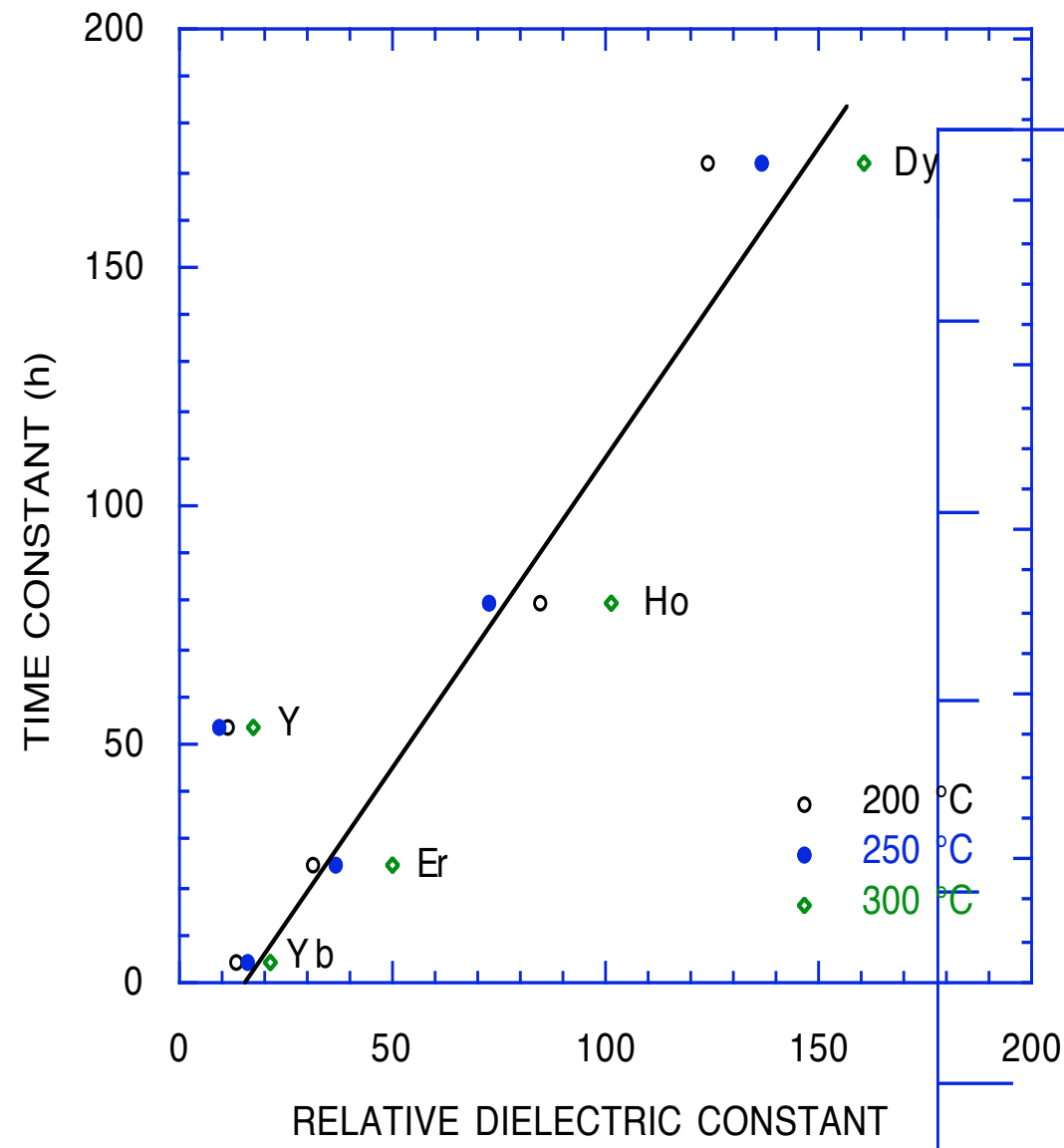

Figure 4.2.7. Relationship between conductivity decay time constant and dielectric constant.

decay occurs by some sort of cation ordering mechanism [50]. However, our results clearly show that the dopant cations affect the kinetics of the ordering process due to their effect on the nature of the vacancy arrangement in the oxygen sublattice. The aging process is governed by vacancy ordering and the displacement of oxygen ions in the lattice.

As described, aging results in the formation of a long range $<111>$ vacancy superlattice and the displacement of oxygen ions from $8 \mathrm{c}$ to $32 \mathrm{f}$ sites. The changes in structural parameters of anion sublattice after 300 hours of aging are shown in Tables 4.2-3 and 4.2-4 for several dopants [46]. From the tables, it is clear that the fraction of oxygen ions in interstitial $32 \mathrm{f}$ positions is higher than that in the regular $8 \mathrm{c}$ sites for both aged and unaged samples. In addition, the fraction of oxygen ions occupying the $32 \mathrm{f}$ positions increases significantly with aging. Therefore, this type of preferential occupancy of oxygen ions in the interstitial $32 \mathrm{f}$ positions is part of the aging process. The higher the occupancy of oxygen ions in the regular $8 \mathrm{c}$ sites, and lower the occupancy of oxygen ions in the intermediate $32 \mathrm{f}$ positions (both before and after aging), the higher the time constant for aging. 


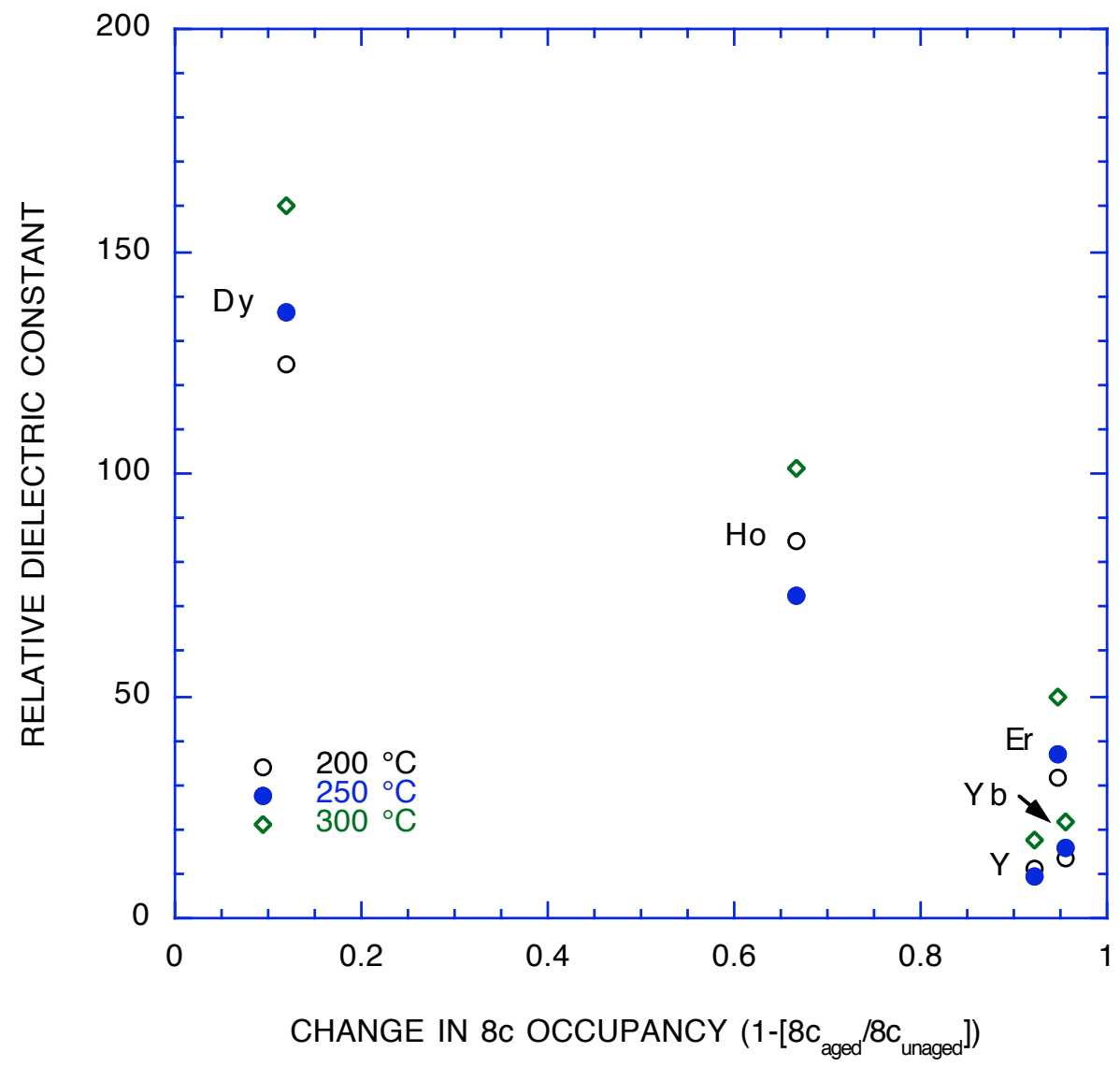

Figure 4.2.8. Relationship between change in occupancy of $8 \mathrm{c}$ sites and dielectric constant.

The oxygen ions in $32 \mathrm{f}$ positions are therefore least stable in Dy doped sample and most stable in $\mathrm{Er}$ and $\mathrm{Yb}$ doped samples. Therefore, the higher the polarizability of the dopant cation (within the range where cubic $\mathrm{Bi}_{2} \mathrm{O}_{3}$ is phase stable) the higher the stability of the disordered structure is below the order- disorder transition. From our results, it is clear that Dysprosium ions do not cause extensive oxygen ion displacements or $<111>$ vacancy ordering even after 300 hours of aging and as a result it exhibits the least conductivity decay.

Since we have previously shown there is a correlation between $\tau$ and the occupancy of $8 \mathrm{c}$ and $32 \mathrm{f}$ sites, and since we know show the linear relationship between $\tau$ and $\kappa$, then there should be a correlation between occupancy of $8 \mathrm{c}$ and $32 \mathrm{f}$ sites and the dielectric constant. This correlation is seen in Fig. 4.2.8. The exact causal relation of this correlation is unclear. That is, the highly polarizable dopants (Dy and Ho) should result in a higher $\kappa$, as shown. This higher polarizability also results in a lower stability of the $32 \mathrm{f}$ site and hence lower change in 8c occupancy. Similarly, the less polarizable dopants $(\mathrm{Er}, \mathrm{Yb}$, and $\mathrm{Y}$ ) should have a lower $\kappa$, and by the same arguments, should have a greater change in $8 \mathrm{c}$ occupancy, as shown. However, these less polarizable dopants all result in a greater than 
$90 \%$ conversion from $8 \mathrm{c}$ to $32 \mathrm{f}$ sites upon aging. Therefore, the environment around the cation is different with these dopants after aging. Further, if the anions are less mobile in the ordered structure, then the induced dipole (at anion vibrational frequencies) associated with the M-O bond should be less. This in turn would result in a lower $\kappa$. This combination of causal effects may explain the more precipitous drop in $\kappa$ associated with the higher changes in $8 \mathrm{c}$ occupancy.

\section{Conclusion}

Due to the high polarizability of the bismuth ion, the pure bismuth oxide has an extensive longrange disorder in the anion sublattice. By doping with less polarizable lanthanide elements, the extent of short range $<111>$ vacancy ordering increases. Consequently, the ionic conductivity of doped cubic bismuth oxides decreases with increasing dopant concentration.

The time dependent decay in conductivity below the order-disorder transition temperature, observed in doped bismuth oxides, occurs due to the long range ordering of oxygen vacancies along $<111>$ directions and the displacement of almost all the oxygen ions from the regular 8c positions to the interstitial 32f positions, also along $<111>$ directions. The kinetics of aging depend upon the polarizability of the dopant cation, with the more polarizable dopants providing the greatest stability against anion ordering and resultant conductivity decay.

\subsubsection{Effect of Oxygen Sublattice Ordering on Interstitial Transport Mechanism and Conductivity Activation Energies}

\section{Effect of Structure on Conductivity Mechanisms in the Disordered Structure}

Based on the structural models discussed above, several theoretical and experimental studies have been performed to study disordered fluorite structures in general, $\delta-\mathrm{Bi}_{2} \mathrm{O}_{3}$ in particular, and the effect of structure on conductivity. Koto et al. studied the structure of $\beta-\mathrm{PbF}_{2}$ and the transport mechanism of the mobile ions through the rigid cation sublattice [47]. They calculated the most favorable conduction pathway by using the interaction energies of the mobile ions in the ionic solid. The minimum energy path for the mobile ions through the static cation sublattice involves motion from one tetrahedral site to the next by following a path that passes close to, but not through the center of the intermediate octahedral site. The potential energy calculations based on this minimum energy path indicate the presence of eight metastable positions near, but slightly displaced from the centers of the octahedral sites. (The off-center displacements predicted by this model almost match with the 
interstitial positions determined by neutron diffraction $[42,43,46]$.) For all studied cases, the energy profile along a direct tetrahedral-tetrahedral path was found to produce a considerably greater activation enthalpy than for the tetrahedral-octahedral-tetrahedral path. This observation is rationalized by the fact that the barrier for migration for a direct $<100>$ jump between two tetrahedral sites is higher due to the rigid cation lattice.

Neutron diffraction studies of cubic bismuth oxides by Battle et al. $[42,43]$ and us [46] show that a significant fraction of oxygen ions occupy the $32 \mathrm{f}$ interstitial positions. These positions are located on the minimum energy conduction pathway calculated by Koto et al. [47]. Therefore, their conduction model calculated for lead fluoride can be applied to the fluorite structure of $\delta-\mathrm{Bi}_{2} \mathrm{O}_{3}$. From these studies, it can be concluded that the interstitial $32 \mathrm{f}$ positions are located immediately adjacent to the transition state or saddle point for the transport of oxygen ions between the regular tetrahedral sites.

Jacobs et al. carried out extensive computational studies to determine the nature of the defect structure of $\delta-\mathrm{Bi}_{2} \mathrm{O}_{3}[48-50]$. They show that it is energetically favorable for the formation of $<110>$ defects in a $<111>$ vacancy arrangement. In addition, the oxygen ions are displaced slightly towards the central octahedral void during their transport between tetrahedral sites. These displacements are not as extensive as those observed by Battle et al. or those predicted by Koto et al.

The neutron diffraction studies of Battle et al. reveal the presence of extensive short range ordering of oxygen ions $[42,43]$. They observed that the extent of short range ordering increases with increasing dopant concentration. They propose that pure bismuth oxide has a tendency to form a $<110>$ vacancy structure rather than a $<111>$ structure because of the high polarizability of the bismuth ion, due its stereo-chemically active lone-pair of electrons. The $<110>$ vacancy arrangement clearly facilitates a greater degree of disorder in the anion sublattice. The dopant rare earth oxides have lower polarizability than bismuth and therefore prefer the $<111>$ vacancy structure. Therefore, in doped bismuth oxides the oxygen vacancies have a greater tendency to align along $<111>$ directions.

Based on the experimental and theoretical calculations, we conclude that the high temperature fluorite structure of bismuth oxide consists of a disordered oxygen sublattice, which has a short range ordering of oxygen vacancies along $<111>$ directions. The tendency for this type of ordering increases with increasing dopant concentration. In addition, the displacement of oxygen ions from the regular $8 \mathrm{c}$ sites to the interstitial $32 \mathrm{f}$ sites plays a significant role in the conductivity mechanism of bismuth oxide. From our neutron diffraction work [46, 48], we have shown that the displacement of oxygen ions occurs simultaneously with vacancy ordering. Thus, it appears there are two types of ordering 
phenomenon in the defect fluorite structure of stabilized cubic $\mathrm{Bi}_{2} \mathrm{O}_{3}$. The ordering of vacancies along $<111>$ directions and the displacement of oxygen ions from the regular to the interstitial sites.

From the above studies, it is clear that the transport mechanism in cubic disordered bismuth oxides involves the occupancy of interstitial sites, and the fraction of oxygen ions occupying these sites affects the conductivity of the material. The interstitial transport path (proposed by Koto et al.) is indicated in Fig. 4.2.9 both for Sillen and Gattow occupancy of 8c sites. The figure illustrates the most energetically favorable path for $<100>$ transport of an oxygen ion from the regular tetrahedral position $8 \mathrm{c}$, through an interstitial $32 \mathrm{f}$ position, towards the central octahedral site (but not through it), through another $32 \mathrm{f}$ position, to a vacant tetrahedral site $(8 \mathrm{c})$ adjacent to the original tetrahedral site.

Obviously, this path has a higher jump distance than a direct $<100>$ jump between the regular tetrahedral sites. However, it is preferred because of the lower energy barrier due to the vacant octahedral site. It should be noted that the only stable occupancy of the oxygen ions along this path is found at $32 \mathrm{f}$ positions [38]. Consequently, the occupancy of oxygen ions in these sites must play a crucial role in the conduction mechanism.

\section{Ordered Structure Below the Transition Temperature}

It has been observed that the conductivity of phase-stabilized bismuth oxides undergoes a decay in conductivity when annealed below $600{ }^{\circ} \mathrm{C}[38-40]$. This decay, termed as aging occurs due to the time and temperature dependent ordering of oxygen ions below the order-disorder transition temperature.

Transformation to the ordered structure could not be detected by XRD because of weak scattering of $x$-rays by oxygen ions. It was, however, observed by TEM diffraction which show the formation of a superlattice $[51,52]$. The kinetics of the ordering phenomenon depend upon the ionic radius of the dopant cation. From the studied compositions, it was shown that the aging rate is least for $\mathrm{Dy}^{3+}$ ion and highest for $\mathrm{Yb}^{3+}$ ion [39]. We have studied several models for the ordered structures and concluded that $\mathrm{a}<111>$ vacancy ordering is most consistent with the TEM diffraction studies [40, 51].

Neutron diffraction experiments indicate that the vacancy ordering process is accompanied by the displacement of nearly all the oxygen ions from the $8 \mathrm{c}$ to $32 \mathrm{f}$ positions $[38,46]$. These displacements were taken into account in the modeling of the ordered structures [40, 51]. It has been discussed in the analysis of neutron diffraction results that a combination of Sillen and Willis models is consistent with the ordered structure [46]. 
(a)

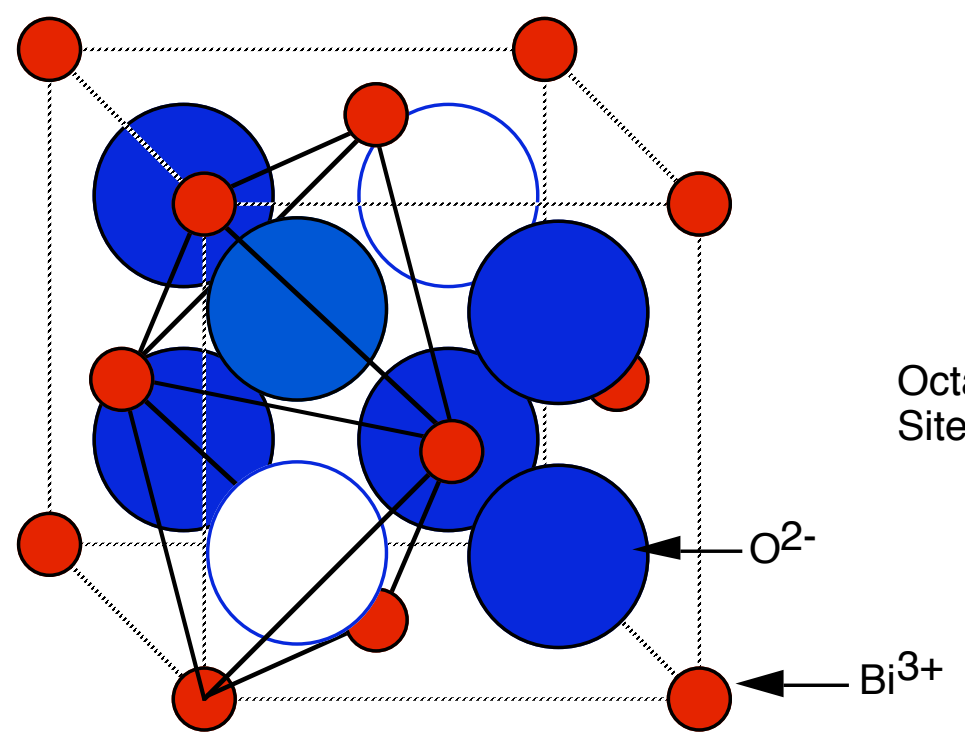

(b)

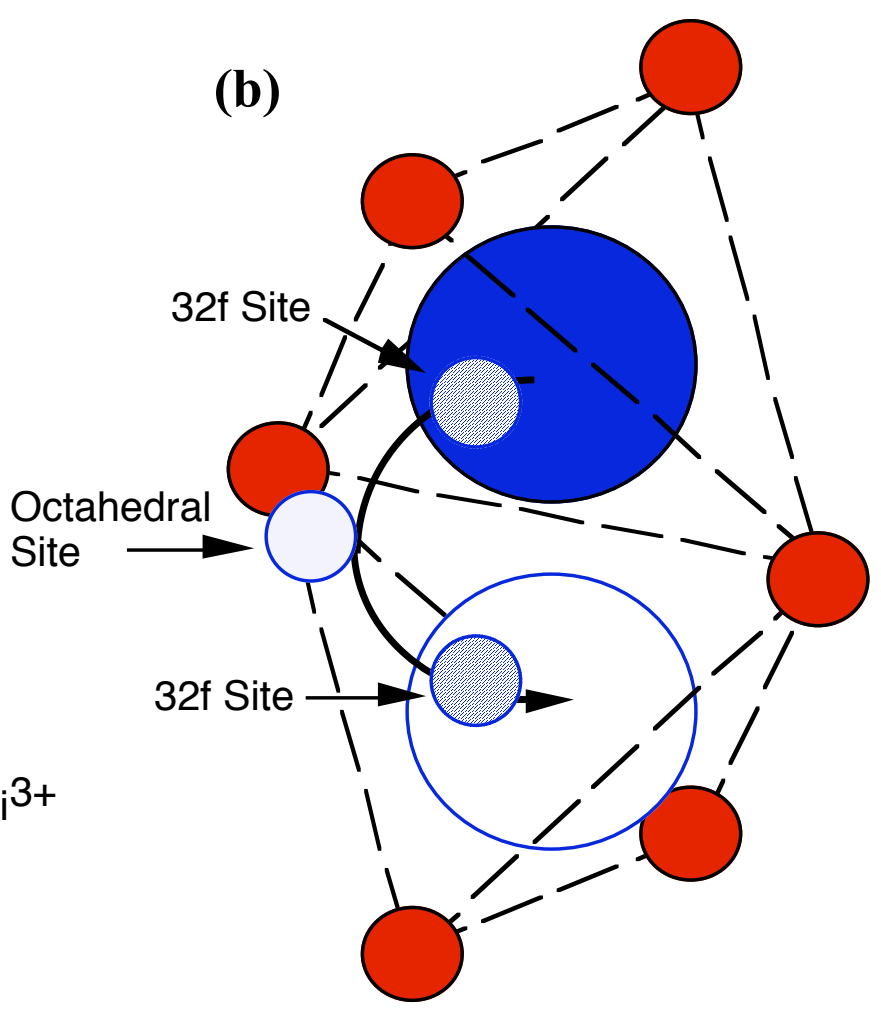

(c)

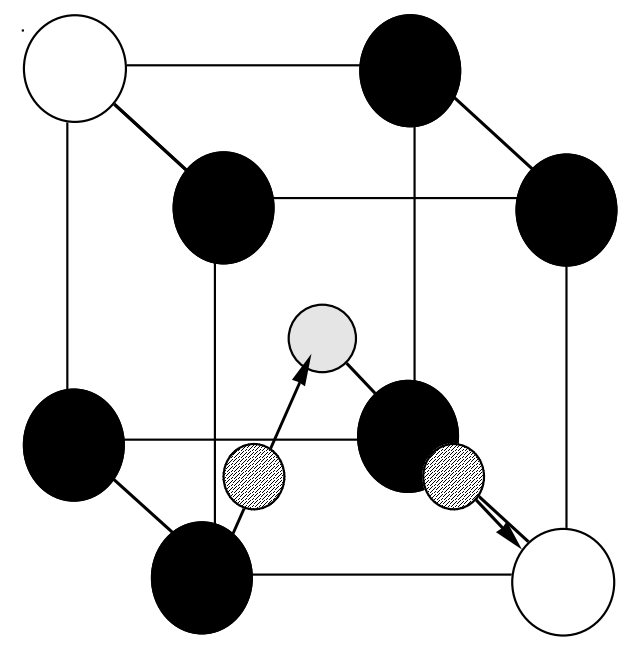

Transport in Ordered Structure

(Sillen Model)

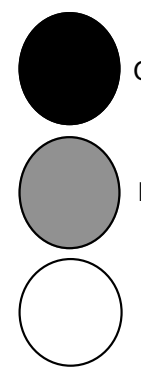
Vacant $8 c$ site

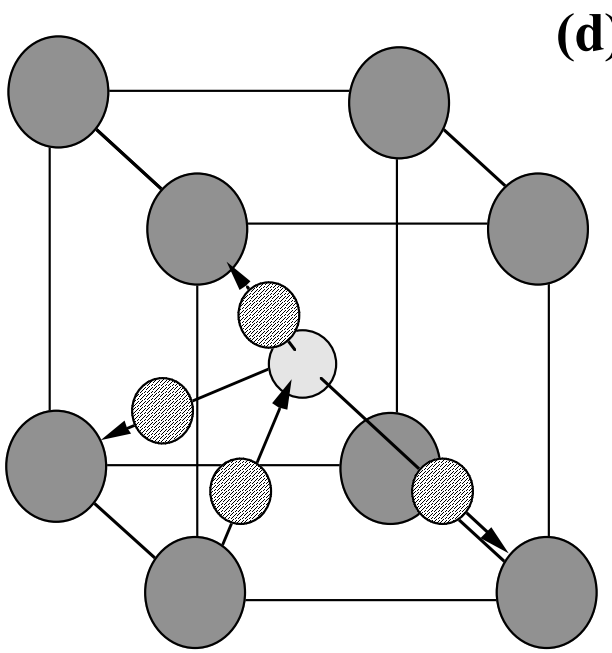

Transport in Disordered Structure

(Gattow Model)

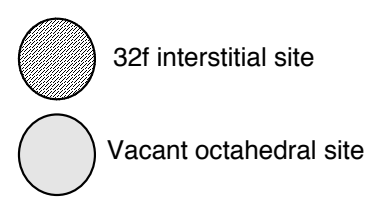

Figure 4.2.9. $\delta-\mathrm{Bi}_{2} \mathrm{O}_{3}$ (a) structure, (b) interstitial transport mechanism, (c) transport in ordered structure (Sillen model), and (d) transport in disordered structure (Gattow model). 
From the above discussion, it is clear that the structure of both the ordered (below $600{ }^{\circ} \mathrm{C}$ ) and the disordered (above $600{ }^{\circ} \mathrm{C}$ ) structures is quite complex and has been a subject of controversy. In order to understand the ionic conductivity of the material, it is imperative to elucidate the effect of structure on conductivity and conductivity activation energy.

In this work, we studied the structure of both ordered and disordered samples of bismuth oxide stabilized with several dopants (Yb, Er, Y, Ho, and Dy). We also determined the conductivity activation energies of each composition both prior to and after aging. Based on these results, we describe a model for an interstitial transport mechanism, whereby oxygen ions move from a filled to an adjacent empty anion site by migration through intermediate interstitial sites. Using the structural information of the compositions, we calculated the conductivity pre-exponentials and compared the values with the observed values to demonstrate the validity of the mechanism.

\section{Experimental}

Conductivity activation energies were determined from plots of $\sigma \mathrm{T}$ vs. 1/T below the orderdisorder transition temperature. For the disordered structures the samples were quenched below the order-disorder transition temperature for measurement, with minimal effect of aging, then heated above the transition temperature to disorder the anion lattice and then quenched again to the next temperature. The aged samples were cooled rapidly from $500{ }^{\circ} \mathrm{C}$ after $300 \mathrm{hr}$ and the activation energies were determined from Arrhenius plots in the temperature range $200-400^{\circ} \mathrm{C}$ where the rate of aging is relatively low. From these experiments, we could compare the activation energies of aged and unaged samples under similar temperature ranges without any significant time dependence to the data.

\section{Results and Discussion}

Anion Ordering and its Effect on Conductivity Activation Energy. It is clear that the polarizability of the cation sublattice affects the nature of distribution of oxygen ions in the anion sublattice. It has been determined by Jacobs et al. that the stability of the intermediate interstitials was lowered by increasing the cation polarizability [48]. This is consistent with our results shown in Tables 4.2-3 and 4.2-4. The fraction of oxygen ions in the $32 \mathrm{f}$ interstitial positions is lowest for the structure doped with the Dy ion, which is the most polarizable among the studied dopants. This clearly has a significant effect on the conductivity mechanism, as discussed in the subsequent sections.

The activation energy values measured for aged and unaged samples with different dopants are shown in Table 4.2-5(a). The activation energies for different compositions of Er are given in Table 
4.2-5(a). The activation energies for the aged samples of 25 DySB, 30 ErSB, 35 ErSB, and 40ErSB were not calculated because of slow kinetics of aging for these compositions. From Table 4.2-5, it is clear that the activation energy is less for the aged samples. This is a surprising result because the conductivity of the aged samples is orders of magnitude less than that of unaged samples. This can however be explained by the concept of the minimum energy path discussed earlier. In the aged samples, oxygen ions occupy the interstitial sites, which are the intermediate states in the minimum energy path of tetrahedral-octahedral-tetrahedral transport. Thus, the conductivity activation energy of the ordered structure is less because most of the oxygen ions are in the intermediate stages of the path.

However, in the ordered structure, transport of oxygen ions is more constrained because of the ordered $<111>$ vacancy arrangement (Fig. 4.2.9, ordered structure). Therefore, the probability of oxygen ions in the $32 \mathrm{f}$ positions to have a neighboring vacant $8 \mathrm{c}$ site in the transport from one tetrahedral site to an adjacent tetrahedral site (in $<100>$ direction) is much lower than that in a disordered structure where all the 8c sites have an equal probability to be vacant or occupied (Fig. 4.2.9, disordered structure). Thus, the number of actual jumps of oxygen ions into vacancies is limited, leading to lower ionic conductivity.

In the disordered structure, the oxygen ions are randomly distributed between the regular and the interstitial sites. The conductivity activation energy is greater due to less oxygen ions in the interstitial sites. However, the transport of oxygen ions into the vacant sites is statistically greater. Due to the

Table 4.2-5. Activation energy, $\mathrm{E}_{\mathrm{A}}$, of (a) $\left(\mathrm{Bi}_{2} \mathrm{O}_{3}\right)_{0.75}\left(\mathrm{M}_{2} \mathrm{O}_{3}\right)_{0.25}$ and (b) $\left(\mathrm{Bi}_{2} \mathrm{O}_{3}\right)_{1-\mathrm{x}}\left(\mathrm{Er}_{2} \mathrm{O}_{3}\right)_{\mathrm{x}}$

\begin{tabular}{lcc}
\hline Composition & $\mathrm{E}_{\mathrm{A}}$ of Unaged Samples $(\mathrm{eV})$ & $\mathrm{E}_{\mathrm{A}}$ of Samples Aged at $500^{\mathrm{O}} \mathrm{C}(\mathrm{eV})$ \\
\hline$(\mathrm{a})$ & 1.23 & 1.10 \\
$\left(\mathrm{Bi}_{2} \mathrm{O}_{3}\right)_{0.75}\left(\mathrm{Yb}_{2} \mathrm{O}_{3}\right)_{0.25}$ & 1.22 & 1.09 \\
$\left(\mathrm{Bi}_{2} \mathrm{O}_{3}\right)_{0.75}\left(\mathrm{Er}_{2} \mathrm{O}_{3}\right)_{0.25}$ & 1.22 & 1.07 \\
$\left(\mathrm{Bi}_{2} \mathrm{O}_{3}\right)_{0.75}\left(\mathrm{Y}_{2} \mathrm{O}_{3}\right)_{0.25}$ & 1.22 & 1.08 \\
$\left(\mathrm{Bi}_{2} \mathrm{O}_{3}\right)_{0.75}\left(\mathrm{Tm}_{2} \mathrm{O}_{3}\right)_{0.25}$ & 1.26 & 1.13 \\
$\left(\mathrm{Bi}_{2} \mathrm{O}_{3}\right)_{0.75}\left(\mathrm{Ho}_{2} \mathrm{O}_{3}\right)_{0.25}$ & 1.19 & \\
$\left(\mathrm{Bi}_{2} \mathrm{O}_{3}\right)_{0.75}\left(\mathrm{Dy}_{2} \mathrm{O}_{3}\right)_{0.25}$ & & \\
$(\mathrm{~b})$ & 1.24 & 1.02 \\
$\left(\mathrm{Bi}_{2} \mathrm{O}_{3}\right)_{0.75}\left(\mathrm{Er}_{2} \mathrm{O}_{3}\right)_{0.15}$ & 1.25 & 1.04 \\
$\left(\mathrm{Bi}_{2} \mathrm{O}_{3}\right)_{0.75}\left(\mathrm{Er}_{2} \mathrm{O}_{3}\right)_{0.2}$ & 1.22 & \\
$\left(\mathrm{Bi}_{2} \mathrm{O}_{3}\right)_{0.75}\left(\mathrm{Er}_{2} \mathrm{O}_{3}\right)_{0.25}$ & 1.22 & \\
$\left(\mathrm{Bi}_{2} \mathrm{O}_{3}\right)_{0.75}\left(\mathrm{Er}_{2} \mathrm{O}_{3}\right)_{0.3}$ & 1.20 & \\
$\left(\mathrm{Bi}_{2} \mathrm{O}_{3}\right)_{0.75}\left(\mathrm{Er}_{2} \mathrm{O}_{3}\right)_{0.35}$ & 1.21 & \\
$\left(\mathrm{Bi}_{2} \mathrm{O}_{3}\right)_{0.75}\left(\mathrm{Er}_{2} \mathrm{O}_{3}\right)_{0.4}$ & & \\
\hline
\end{tabular}


disordered nature of the lattice the number of possible jump directions is higher as shown in Fig. 4.2.9 (disordered structure). This leads to a significantly higher conductivity.

The migration of the oxygen ions in the lattice involves breaking of ionic bonds between the cations and the anions. Therefore, the conductivity activation energy depends upon the strength of the ionic bond. The activation energy should therefore be higher for the structure with stronger ionic bond. The force constant, $k$, for an ionic bond is given by

$$
k=\frac{2 z_{1} z_{2}}{\pi \varepsilon_{0} r_{0}^{3}} e^{2}
$$

where $z_{1} e$ and $z_{2} e$ are the effective charges of anion and cation respectively ( $2 e$ and $3 e$ in our case), and $r_{0}$ is the inter-atomic distance, or the bond distance. From the above equation, it is clear that the force constant depends upon the equilibrium distance between the cations and the anions. This distance will vary with the nature of the distribution of oxygen ions in the anion sublattice.

In the fluorite structure, if the oxygen ion occupies the regular $8 \mathrm{c}$ site, the bond distance would be $\sqrt{ } 3 / 4 \mathrm{a}$, and if the oxygen ion occupies the interstitial $32 \mathrm{f}$ position, the bond distance would be 0.606a. From the fractional distribution of oxygen ions in 8c and 32f sites (Tables 4.2-3 and 4.2-4), the equilibrium bond distances in the ordered and disordered structure have been determined. The calculated bond lengths and the force constants of the bonds in ordered and disordered structures are shown in Table 4.2-6. For all the cases, the force constants for the disordered structures is higher than those of the corresponding ordered structure, consistent with their observed higher activation energies.

The thermal vibrational frequency of the atoms also depends on the bond strength. The frequency of vibration of atoms, $v$, is given by

Table 4.2-6. Calculated bond strength and vibrational frequencies for ordered and disordered structures

\begin{tabular}{lcccc}
\hline Dopant & Structure & Bond Length $(\AA)$ & Force Constant $(\mathrm{N} / \mathrm{m})$ & Vibrational Frequency $(\mathrm{GHz})$ \\
\hline $\mathrm{Yb}$ & Disordered & 2.644 & 597.83 & 9810 \\
& Ordered & 2.966 & 423.49 & 8260 \\
$\mathrm{Ee}$ & Disordered & 2.624 & 611.60 & 10020 \\
& Ordered & 2.965 & 423.92 & 8340 \\
$\mathrm{Y}$ & Disordered & 2.625 & 610.90 & 12220 \\
& Ordered & 2.963 & 424.78 & 10200 \\
$\mathrm{Ho}$ & Disordered & 2.597 & 630.88 & 10210 \\
& Ordered & 2.879 & 463.06 & 8750 \\
$\mathrm{Dy}$ & Disordered & 2.581 & 642.68 & 10350 \\
& Ordered & 2.639 & 601.23 & 10010 \\
\hline
\end{tabular}




$$
v=2^{-1} \pi^{-1} k^{\frac{1}{2}} M_{r}^{-\frac{1}{2}}
$$

where $M_{r}$ is the effective mass of the oscillating system, given by $M_{r}=m_{1} m_{2} /\left(m_{1}+m_{2}\right)$, where $m_{1}$ and $m_{2}$ are the respective atomic weights of anion and cation. From the calculated values of force constants, the vibrational frequencies of oxygen ions have been calculated and are shown in Table 4.26.

From the table, it is clear that the vibrational frequency in the disordered structures is higher than that in the corresponding ordered structures by about 15 percent. This is as expected and is consistent with the higher slope obtained for the disordered structures both in the Differential Thermal Analysis (DTA) and the mean square atomic displacement of oxygen ions versus temperature [46].

Effect of Structure on Conductivity Pre-Exponential Factor. Nowick et al. [55] computed the pre-exponential term in conductivity for various families of ionic conductors. According to them, the pre-exponential term will vary with three possible structural arrangements, two of which are pertinent to this discussion. First, the structure is completely disordered and the defects are equally likely to occupy any site at any instant of time; this is the case with the unaged structure. Second, most of the carriers are bound at specific defect sites, while only a small fraction of carriers are free to move; this is the case of aged structure, in which the majority of oxygen ions are not mobile because of lack of vacancies at nearest neighbor positions. For the aged structure, the value of the pre-exponential will be less than that for the disordered case.

The pre-exponential also depends on the square of the jump distance, which is lower for aged sample. Due to the ordered structural arrangement of the mobile anion sublattice, and the occupancy of oxygen ions in the intermediate stages of conduction paths, the conductivity activation energy would be lower than that of the disordered structure, what we experimentally observed.

Almost all the carriers are bound to various traps and only those that are released contribute to conductivity. Fung et al. use this theory to explain aging phenomenon. They argue that dopant cations become ordered and act as traps for oxygen ions [52]. In this case, the pre-exponential will be greater in the ordered structure than that in the disordered case, and the activation energy will be higher, contrary to the observed results (Table 4.2-5). Therefore, it is not possible to explain the lower conductivity and lower conductivity activation energy of the ordered structure without considering vacancy ordering and the occupancy of oxygen ions in interstitial sites.

The temperature dependence of conductivity is given by 


$$
\sigma T=A \exp \left(-E_{A} / k_{B} T\right)
$$

where $\sigma$ is the oxygen ion conductivity, $T$ is the temperature, $E_{A}$ is the activation energy, and $k_{B}$ is the Boltzmann Constant. The pre-exponential term $A$ is given by

$$
A=\frac{n \lambda^{2}(z e)^{2} v c_{i}}{6 V k_{B}}
$$

where $n$ is the number of directions in which the jump may occur, $\lambda$ is the jump distance, ze is the electronic charge of the conducting species, $v$ is the pre-exponential of the jump frequency, $c_{i}$ is the concentration of the charge carriers, and $V$ is the volume of the unit cell. The variables in Eq. (4.2.5) depend primarily on the structure of the anion sublattice. From the neutron diffraction data (Tables 4.2-3 and 4.2-4) and the structural models for the ordered structure, we can determine these variables for ordered and disordered structures, and compare the pre-exponentials for the two structures.

Jump Frequency. The pre-exponential of the jump frequency, $v$ is given by

$$
v=v_{\mathrm{o}} \exp \left(\Delta \mathrm{S}_{\mathrm{m}} / \mathrm{k}\right)
$$

where $v_{\mathrm{o}}$ is the characteristic vibrational frequency (shown in Table 4.2-6) and $\Delta \mathrm{S}_{\mathrm{m}}$ is the entropy of activation. The values of $\Delta \mathrm{S}_{\mathrm{m}} / \mathrm{k}$ have been calculated to be in the range of 2 to 3 for simple defect systems [55]. Since this factor causes no significant change in the order of the pre-exponential values, it has been ignored in our calculations. The values of $v_{0}$ are typically in of the order of $10^{13}$ consistent with our calculated values [55].

Jump Distance. Figure 4.2.10 illustrates the positions and the atomic coordinates of the sites important in the conduction mechanism. The figure is not drawn to scale with respect to the ionic sizes of the ions and is used primarily for calculating the jump distances in the unit cell. From the figure, we can see that a single ionic jump constitutes the movement of an oxygen ion from position A to position E. We have discussed previously that the most favorable path for this event is $A \Rightarrow B \Rightarrow C \Rightarrow D \Rightarrow E$. However, from Tables 4.2-3 and 4.2-4, we can see that there is a significant fraction of oxygen ions in $32 \mathrm{f}$ sites (positions B and D) in the disordered structures, and the majority of oxygen ions occupy those positions in the ordered structures. From Fig. 4.2.10, we can see that the length of the body diagonal $<111>$ is $\mathbf{a} \sqrt{ } 3$, where $\mathbf{a}$ is the lattice parameter. The length of $\mathrm{AC}$ is $\mathbf{a} \sqrt{ } 3 / 4(=0.433 \mathrm{a})$. Therefore, the jump distance from the $8 \mathrm{c}$ site at $\mathrm{A}$ to the $8 \mathrm{c}$ site at $\mathrm{E}$ is $\mathbf{a} \sqrt{3} / 2$ which is $0.866 \mathrm{a}$. The distance between B $(0.313,0.313,0.313)$ to $C(0.5,0.5,0.5)$ is $(\mathrm{a} \sqrt{3} / 8) \cdot(0.375 / 0.313)$ which is $0.260 \mathrm{a}$. Therefore, the jump distance from the $32 \mathrm{f}$ site at $\mathrm{B}$ to the $8 \mathrm{c}$ site at $\mathrm{E}$ is $0.260 \mathrm{a}+0.433 \mathrm{a}$, which equals $0.693 \mathrm{a}$. 


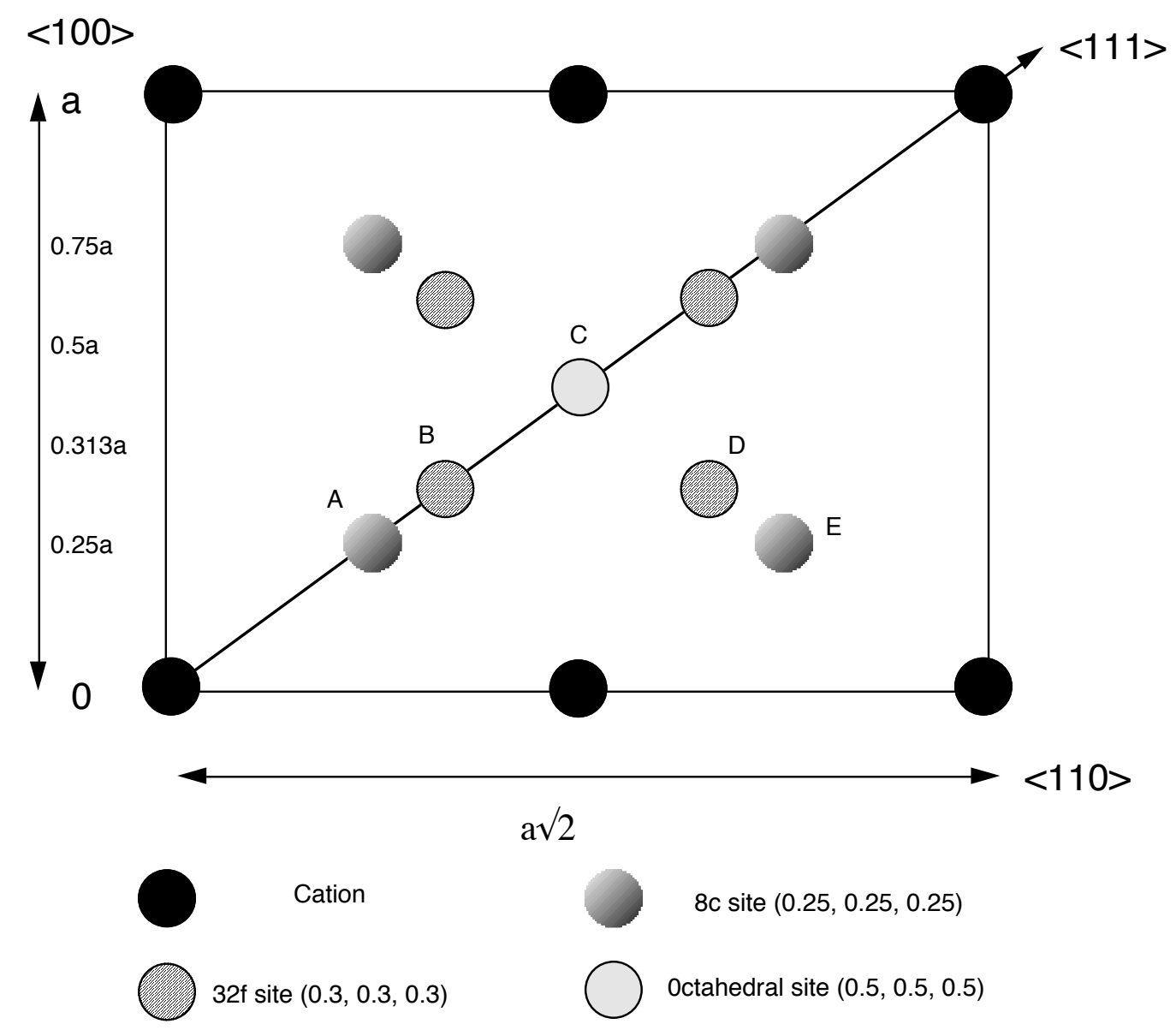

Figure 4.2.10. Jump from one tetrahedron to an adjacent empty tetrahedron in $<100>$ direction

Figure 4.2.9 illustrates the mechanism of a $<100>$ jump from an occupied $8 \mathrm{c}$ site to an adjacent empty $8 \mathrm{c}$ site. Each of these tetrahedral positions is surrounded by a cation tetrahedron with four $32 \mathrm{f}$ positions, one at each face of the tetrahedron. For our calculations we will consider a jump to have occurred only if an anion moves from a tetrahedron, either from the central 8c or associated $32 \mathrm{f}$ positions, to an adjacent vacant tetrahedron. Referring to Fig. 4.2.10, this means that a jump will have occurred if an anion moves from either position A or B to position E, but not if an anion moves from position $\mathrm{D}$ to position $\mathrm{E}$ since the latter is only displacement of the anion within the same tetrahedron.

From the calculations above, it is clear that if the oxygen ion is in an $8 \mathrm{c}$ site, the effective jump distance is $0.866 \mathrm{a}$. If it is in the $32 \mathrm{f}$ site displaced from the same $8 \mathrm{c}$ site, the jump distance to the nearest neighbor empty tetrahedral site is 0.693a. From Tables 4.2-3 and 4.2-4, we see that there is an occupancy of oxygen ions in both $8 \mathrm{c}$ and $32 \mathrm{f}$ sites. So we have to take into account, the fraction of oxygen ions in these two sites, and the respective jump distances to calculate the effective jump distance. 
Jump Directions. From our previous work [51] we determined the most likely model for an ordered structure with oxygen ions displaced from the $8 \mathrm{c}$ to $32 \mathrm{f}$ positions. A section of the model (four unit cells) is shown in Fig. 4.2 .11 (without displacements) and involves a $<111>$ vacancy superlattice similar to the Sillen model shown in Fig. 4.2.9. However, from Fig. 4.2.11, it can be observed that this model also includes a $<110>$ ordering of vacancies. As discussed in previous sections, this $<110>$ ordering is preferred by bismuth ions. Therefore, this type of an ordered structure (with both $<111>$ and $<110>$ vacancy ordering) is most likely to form.

In Fig. 4.2.11, each oxygen ion has two nearest neighbor vacancies along the $<100>$ directions. Thus, it appears that the number of possible jump directions in this ordered structure is two. However, if we consider the positional ordering in the structure, i.e., the displacement of each of the oxygen ions from an $8 \mathrm{c}$ site to an adjacent $32 \mathrm{f}$ site, the number of jump directions would be limited to one. This is seen in Fig. 4.2.12, which shows a two dimensional section of the superlattice. Let us consider the jump directions of an oxygen ion $\mathrm{O} 1$ which has only occupancy ordering. It has two nearest neighbor vacancies, V1 and V2 in $<100>$ directions. Thus, an oxygen ion at $\mathrm{O} 1$ is likely to have two possible jump directions: $\mathrm{O} 1 \Rightarrow \mathrm{C} 1 \Rightarrow \mathrm{V} 1$ and $\mathrm{O} 1 \Rightarrow \mathrm{C} 2 \Rightarrow \mathrm{V} 2$ as shown in Fig. 4.2.12(a). In Fig. 4.2.12(b), the displacement of oxygen ions into $32 \mathrm{f}$ sites (positional ordering) is also shown. Here, the oxygen ion at $\mathrm{O} 1$ is displaced to the interstitial site $\mathrm{f} 1$. Thus, the only possible jump direction is $\mathrm{f} 1 \Rightarrow \mathrm{C} 1 \Rightarrow \mathrm{V} 1$. The other direction would not constitute a single jump, because the oxygen at f1 would have to first come back to O1. Neutron diffraction studies showed that the occupancy of oxygen ions in $8 \mathrm{c}$ sites is negligible. Thus, it is unlikely to have two jump directions in the ordered structure. Due to occupancy $(<111>$ and $<110>$ vacancy ordering) and positional ordering ( $<111>$ displacements of oxide ions), the number of possible jump directions is limited to one. In the disordered case, the number of possible jump directions is six with two equivalent paths for each jump resulting in twelve equivalent paths.

Charge and Concentration of Charge Carriers. In the disordered structure, all the oxygen ions are equally likely to perform a jump because there is an equal probability for each of the eight $8 \mathrm{c}$ sites to be vacant at any instant. Therefore, the mole fraction of charge carriers in the disordered case is 1 , because all the oxygen ions are mobile charge carriers. However, since each site is occupied by only $3 / 4$ oxygen ion the effective charge carried is only $1.5 \mathrm{e}$.

For the ordered structure, (Figs. 4.2.9 and 4.2.11) we can see that two oxygen ions (out of six) per unit cell can carry out a successful jump to a nearest neighbor tetrahedral site in the $<100>$ directions. 


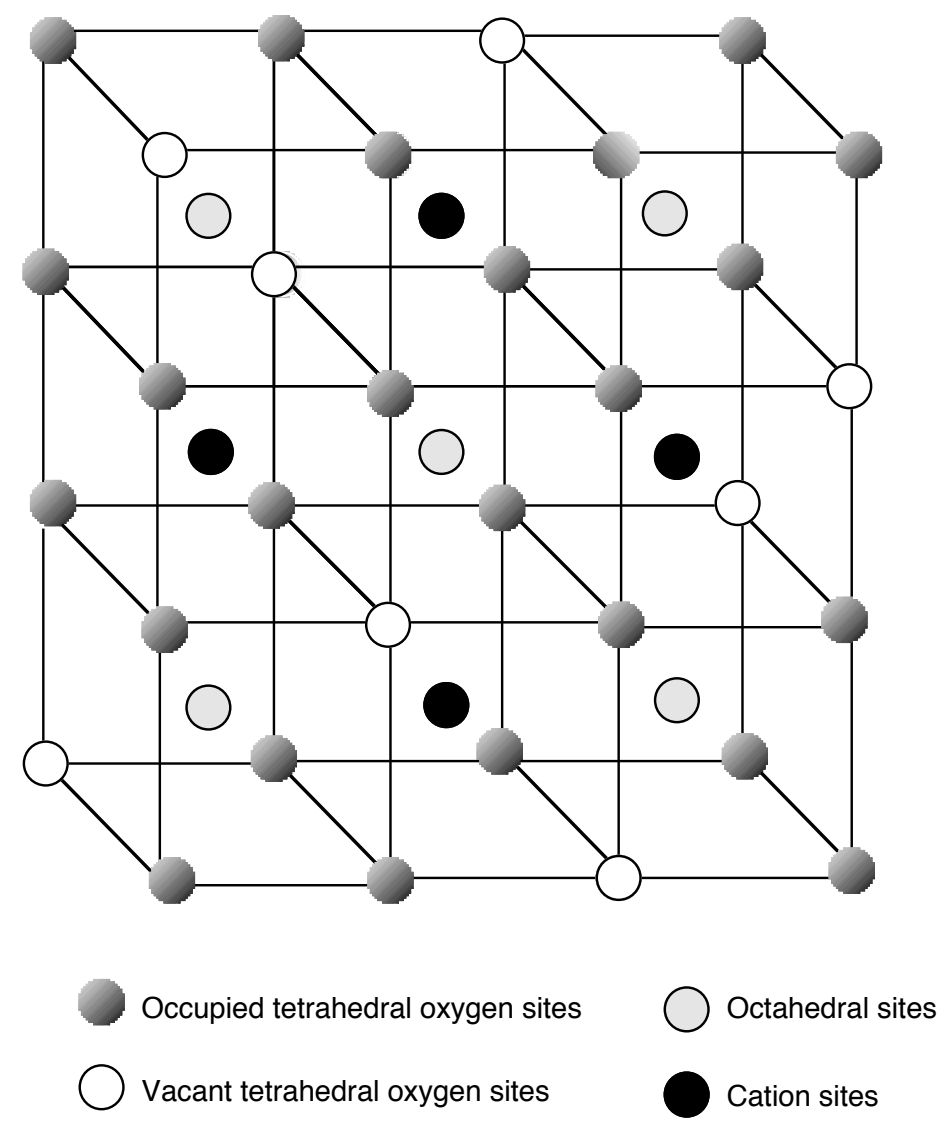

Figure 4.2.11. Vacancy superlattice

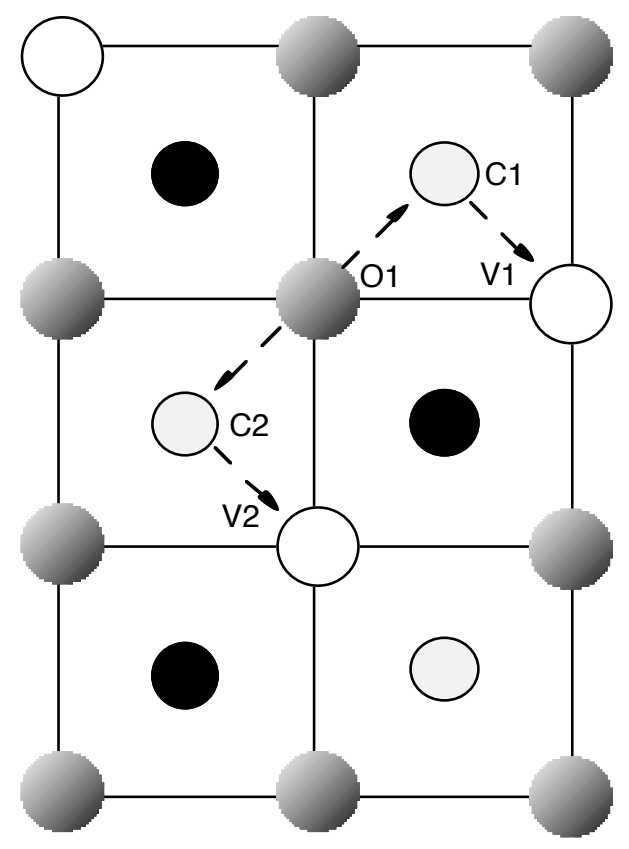

a. Occupancy ordering

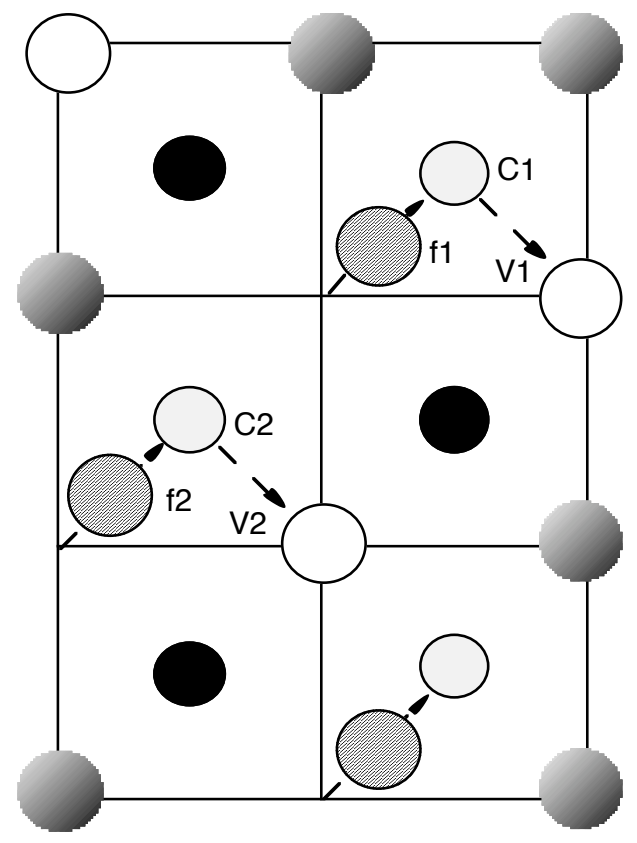

b. Occupancy and positional ordering

Figure 4.2.12. Sketch of jump directions in ordered structure with occupancy and positional ordering 
Table 4.2-7. Comparison of variables affecting the pre-exponential factor, A.

\begin{tabular}{ccc}
\hline & Disordered Structure & Ordered Structure \\
\hline$\lambda$ & 6 & {$\left[0.866 \cdot 8 \mathrm{c}_{\text {unaged }}+0.693 \cdot 32 \mathrm{f}_{\text {unaged }}\right] \mathrm{a} / 3$} \\
$\mathrm{n}$ & $\left.6 \mathrm{c}_{\text {aged }}+0.693 \cdot 32 \mathrm{f}_{\text {aged }}\right] \mathrm{a} / 3$ \\
$\mathrm{Ze}$ & $1.5 \mathrm{e}$ & 1 \\
$\mathrm{C}_{\mathrm{i}}$ & 1 & $2 \mathrm{e}$ \\
\hline
\end{tabular}

Therefore, the mole fraction of charge carriers in this case is 0.333 . Since there is no fractional occupancy of sites, the effective charge carried is $2 \mathrm{e}$.

Pre-Exponential. The variables appearing in the equation for the pre-exponential for both ordered and disordered structures are summarized in Table 4.2-7 except for $v$, whose values are shown in Table 4.2-6. From the tables, it is clear that there is a significant difference in the parameters that affect the pre-exponential between the ordered and disordered structures. The $8 \mathrm{c}$ and $32 \mathrm{f}$ terms shown in Table 4.2-7 are the fraction of oxygen ions in those two sites respectively. These fractions were taken from the occupancies of oxygen ions shown in Table 4.2-6.

The jump distance, $\lambda$ shown in Table 4.2-7 will vary with the lattice parameter (a) and the distribution of the oxygen ions between the $8 \mathrm{c}$ and $32 \mathrm{f}$ positions. The lattice parameters and the corresponding actual jump distances for each of the dopants are shown in Table 4.2-8. Table 4.2-8 shows that the difference between the jump distances of ordered and disordered structures is highest for $\mathrm{Yb}$ and Er doped structures, and lowest for the Dy doped structures. This clearly is consistent with the difference in kinetics of aging for structures with different dopants. In addition, we know that the ordered structure does not form completely for the case of Dy. That is, while the occupancy of $8 \mathrm{c}$ vs. $32 \mathrm{f}$ sites (from neutron diffraction data) is accounted for in our calculations, the fact that the larger radii and more polarizable dopants result in ordering at a much slower rate means that the $<111>$ ordering of vacancies may not have fully occurred after 300 hours at $500^{\circ} \mathrm{C}$. However, the calculations were carried out for this case assuming the same model for the sake of comparison.

Table 4.2-8. Calculated jump distances, $\lambda$, in ordered and disordered structures

\begin{tabular}{cccc}
\hline Dopant & Lattice Parameter $(\AA)$ & $\lambda$ in the Disordered Structure, $(\AA)$ & $\lambda$ in the Ordered Structure, $(\AA)$ \\
\hline $\mathrm{Yb}$ & 5.474 & 3.755 & 3.433 \\
$\mathrm{Er}$ & 5.484 & 3.787 & 3.445 \\
$\mathrm{Y}$ & 5.492 & 3.795 & 3.457 \\
$\mathrm{Ho}$ & 5.493 & 3.825 & 3.543 \\
$\mathrm{Dy}$ & 5.501 & 3.849 & 3.792 \\
\hline
\end{tabular}


The calculated pre-exponentials based on the above approach are shown in Table 4.2-9(a). From the table, it is clear that the conductivity pre-exponential values of ordered structures are an order of magnitude less than those of the disordered structures. The conductivity pre-exponentials (A) were also measured from the Arrhenius plots of ordered and disordered structures in the temperature range $200{ }^{\circ} \mathrm{C}-400{ }^{\circ} \mathrm{C}$ and are shown in Table 4.2-9(b). It is observed that the measured pre-exponentials for the ordered structures are lower than those for the disordered structures and they are of the same order of magnitude as those calculated from the interstitial transport model (Table 4.2-9). There is clearly some difference between the calculated and observed values. This could be due to the approximate nature of the model in which several assumptions had to be made to simplify the calculations. For example, the fraction of oxygen ions occupying the $48 \mathrm{i}$ sites was neglected for the calculation of jump distances and the entropy of activation was neglected in the calculation of pre-exponentials. Further investigations are necessary to refine this model. However, it can be observed from Table 4.2-9 that the ratios of the calculated pre-exponentials of ordered to disordered structures is in close agreement to the corresponding ratios of the measured pre-exponentials indicating the validity of this approach.

Mechanistically, the preferential occupancy of oxygen ions in the $32 \mathrm{f}$ sites leading to a lower conductivity activation energy is consistent with the theoretical calculations of Koto et al. for ionic transport in the fluorite structure [47]. Mathematically, the lower value of the pre-exponential term, A, of the ordered structure results in a lower value of activation energy, Eq. (4.2.4), even though the conductivity is much lower. Therefore, the interstitial transport mechanism explains the anomalous decrease in activation energies for the ordered structures.

Table 4.2-9. (a) Calculated and (b) Measured pre-exponentials for unaged and aged $\left(\mathrm{Bi}_{2} \mathrm{O}_{3}\right)_{0.75}\left(\mathrm{M}_{2} \mathrm{O}_{3}\right)_{0.25}$

\begin{tabular}{|c|c|c|c|}
\hline Dopant & $\mathrm{A}_{\text {Disordered }} \times 10^{5} \mathrm{SK} / \mathrm{cm}$ & A $_{\text {Ordered }} \times 10^{5} \mathrm{SK} / \mathrm{cm}$ & $\mathrm{A}_{\text {Ordered }} / \mathrm{A}_{\text {Disordered }}$ \\
\hline \multicolumn{4}{|c|}{ ston } \\
\hline $\mathrm{Yb}$ & 34.33 & 2.40 & 0.069 \\
\hline $\mathrm{Er}$ & 36.36 & 2.46 & 0.067 \\
\hline $\mathrm{Y}$ & 44.35 & 3.02 & 0.068 \\
\hline Ho & 37.61 & 2.72 & 0.072 \\
\hline Dy & 38.44 & 3.54 & 0.092 \\
\hline \multicolumn{4}{|l|}{ (b) } \\
\hline $\mathrm{Yb}$ & 93.88 & 6.73 & 0.071 \\
\hline $\mathrm{Er}$ & 52.39 & 1.98 & 0.037 \\
\hline $\mathrm{Y}$ & 114.78 & 9.54 & 0.083 \\
\hline Ho & 42.45 & 3.59 & 0.084 \\
\hline Dy & 62.67 & 4.89 & 0.078 \\
\hline
\end{tabular}




\section{Conclusion}

The ionic transport mechanism in the Fluorite structure involves a path that is more complicated than a simple hopping mechanism between the regular tetrahedral sites along $<100>$ directions. This is due to the presence of vacant octahedral positions which lead to more favorable paths involving the displacement of oxygen ions towards the central vacant position along the $<111>$ directions. This has been confirmed experimentally in $\delta-\mathrm{Bi}_{2} \mathrm{O}_{3}$ by neutron diffraction studies, which show extensive displacements of oxygen ions along the $<111>$ directions. Due to the disordered defect fluorite structure of cubic bismuth oxide, this mechanism leads to an exceptionally high ionic conductivity.

The time dependent decay in conductivity below the order-disorder transition temperature, observed in doped bismuth oxides, occurs due to the long range ordering of oxygen vacancies along $<111>$ directions and the displacement of almost all the oxygen ions from the regular 8c positions to the interstitial $32 \mathrm{f}$ positions, also along $<111>$ directions. The kinetics of aging depend upon the ionic radius and polarizability of the dopant cation. The low temperature conductivity activation energies of the ordered bismuth oxides are lower than those of the corresponding disordered cases. This is due to the structural changes in the mobile anion sublattice. The calculated and measured pre-exponentials for the disordered structures are higher than those of the corresponding ordered structures for all the studied compositions. Therefore, the observed reduction in activation energies can be explained by oxygen vacancy ordering and the displacement of oxygen ions to the intermediate states in the conduction path. The activation energy results and the neutron diffraction results are consistent with the general model for interstitial transport mechanism in Fluorite structures. This study confirms the fact that the distribution of oxygen ions, both in terms of occupancy (ordering of vacancies) and displacement (interstitial positions), affects the conductivity of cubic bismuth oxides.

\subsubsection{Development of Highest Conductivity $\mathrm{Bi}_{2} \mathrm{O}_{3}$-Based Electrolyte}

Our results have shown that the dopant cations affect the nature of the vacancy arrangement in the oxygen sublattice [56 - 61] and the polarizability of the cation affects the stability of the disordered oxygen sublattice [62]. These results showed that the most polarizable of the lanthanide dopants, Dy, provided the greatest stability for the disordered anion lattice [62]. Among the potential dopants to stabilize the fluorite structure of $\mathrm{Bi}_{2} \mathrm{O}_{3}, \mathrm{~W}$ has the highest polarizability and therefore should also provide stability for the disordered anion lattice. Moreover, based on the results of Battle et al [56], the high polarizability of Dy and W should result in high anion mobility. 
Takahashi et al [63] reported that $\mathrm{WO}_{3}$ stabilized $\mathrm{Bi}_{2} \mathrm{O}_{3}$ has high conductivity, but the fcc phase was stable only over a narrow composition range (22- $28 \mathrm{~mol} \% \mathrm{WO}_{3}$ in $\mathrm{Bi}_{2} \mathrm{O}_{3}$ ). Verkerk and Burggraaf [64] investigated the phase stability and oxygen ion conductivity of $\mathrm{Dy}_{2} \mathrm{O}_{3}$ stabilized $\mathrm{Bi}_{2} \mathrm{O}_{3}$. They found that the cubic structure was stable at low temperatures for the solid solutions containing $28.5-50 \% \mathrm{Dy}_{2} \mathrm{O}_{3}$. In both investigations it was found that the highest conductivity was obtained for the lowest dopant concentration that would stabilize the fcc phase $\left(\left(\mathrm{BiO}_{1.5}\right)_{0.88}\left(\mathrm{WO}_{3}\right)_{0.12}\right.$ and $\left.\left(\mathrm{BiO}_{1.5}\right)_{0.715}\left(\mathrm{DyO}_{1.5}\right)_{0.285}\right)$. Therefore, based on the cation ratio the fcc structure can be stabilized with $\mathrm{W}$ at a lower dopant concentration than with Dy. In addition, studies $[65,66]$ have shown that the face-centered cubic structure in the $\mathrm{Bi}_{2} \mathrm{O}_{3}$-based solid solutions can be stabilized down to room temperature with two rare earth oxide dopants with smaller total dopant concentrations. Since smaller dopant concentrations have higher conductivity, this results in an increase in conductivity, especially in the lower temperature regions. Therefore, we tried doping $\mathrm{Bi}_{2} \mathrm{O}_{3}$ with both $\mathrm{Dy}_{2} \mathrm{O}_{3}$ and $\mathrm{WO}_{3}$ (DyWSB), the highly polarizable dopants, in order to obtain a structurally stable electrolyte with high conductivity.

\section{Experimental}

Disks of $\mathrm{Dy}_{2} \mathrm{O}_{3}-\mathrm{WO}_{3}-\mathrm{Bi}_{2} \mathrm{O}_{3}$ were fabricated by solid state reaction for conductivity measurements. Disks of $\left(\mathrm{BiO}_{1.5}\right)_{0.8}\left(\mathrm{ErO}_{1.5}\right)_{0.2}$ (henceforth called 20ESB) were also prepared for comparison of conductivity results. Six different compositions were investigated: $\left(\mathrm{BiO}_{1.5}\right)_{0.87}\left(\mathrm{DyO}_{1.5}\right)_{0.10}\left(\mathrm{WO}_{3}\right)_{0.03},\left(\mathrm{BiO}_{1.5}\right)_{0.88}\left(\mathrm{DyO}_{1.5}\right)_{0.08}\left(\mathrm{WO}_{3}\right)_{0.04},\left(\mathrm{BiO}_{1.5}\right)_{0.89}\left(\mathrm{DyO}_{1.5}\right)_{0.06}\left(\mathrm{WO}_{3}\right)_{0.05}$, and $\left(\mathrm{BiO}_{1.5}\right)_{0.83}\left(\mathrm{DyO}_{1.5}\right)_{0.17-\mathrm{x}}\left(\mathrm{WO}_{3}\right)_{\mathrm{x}}($ where $\mathrm{x}=0.05,0.08,0.12)$. The latter 3 compositions with a fixed $17 \%$ total dopant content are 12Dy5WSB, 9Dy8WSB and 5Dy12WSB. The former 3 compositions with a total dopant content between $11-13 \%$ are 10Dy3WSB, 8Dy4WSB and 6Dy5WSB.

Powders of $\mathrm{Bi}_{2} \mathrm{O}_{3}$ (99.9995\% pure), $\mathrm{Er}_{2} \mathrm{O}_{3}$ (99.99\% pure), $\mathrm{Dy}_{2} \mathrm{O}_{3}$ (99.99\% pure) and $\mathrm{WO}_{3}$ (99.8\% pure), from Alfa Aesar, were mixed and ball-milled with zirconia ball media in a high-density polyethylene bottle for 20 hours. The mixed powders were calcined at $800^{\circ} \mathrm{C}$ for 16 hours. Agglomerated powders were ground again using mortar and pestle and pressed into a disk-shaped $0.635 \mathrm{~cm}$ die using $2 \times 10^{5} \mathrm{~N} / \mathrm{m}^{2}$. Poly vinyl alcohol was used as a binder and stearic acid mixed with reagent alcohol as a lubricant. The subsequent green bodies were sintered in air at $890^{\circ} \mathrm{C}$ for 16 hours. Powder X-ray diffraction (XRD) data, using $\mathrm{CuK} \alpha$ radiation with a nickel filter, were obtained at room temperature for phase analysis and results confirmed by neutron diffraction [67]. All compositions were found to be fcc structure; lattice parameters of selected compositions are shown in Table 4.2-10. 
Table 4.2-10. Lattice parameters from [67].

$\begin{array}{cc}\text { Composition } & \text { Lattice Parameter }(\AA) \\ \text { 6Dy5WSB } & 5.576(1) \\ \text { 8Dy4WSB } & 5.567(1) \\ \text { 10Dy3WSB } & 5.554(1)\end{array}$

After sintering, the specimens were polished to acquire an even surface and the desired thickness $(2 \mathrm{~mm})$. Engelhard gold paste mixed with isopropanol was brushed onto both sides of the electrolytes, and the organic additives were evaporated at $127^{\circ} \mathrm{C}$ for $30 \mathrm{~min}$. The electrodes were then sintered at $800^{\circ} \mathrm{C}$ for 30 min. Gold wires (99.95\% pure, DFG), spot-welded with circular gold mesh ( $99.9 \%$ pure, 100 mesh, DFG), were attached to the cells using Au paste and sintered again at $800{ }^{\circ} \mathrm{C}$ for $30 \mathrm{~min}$.

Impedance using the two probe method was measured with a Solartron 1260 over the frequency range of $0.1 \mathrm{~Hz}$ to $1 \mathrm{MHz}$. The frequency response analyzer was interfaced to a computer using Zplot ${ }^{\mathrm{TM}}$ software. Due to the small sample impedances at high temperature a nulling technique was necessary to remove any artifacts caused by inductive responses of the test leads and the equipment. The impedance of the leads without a sample was obtained and subtracted from the sample (plus lead) measurements. The measurements were taken between $200{ }^{\circ} \mathrm{C}$ and $800{ }^{\circ} \mathrm{C}$ in air. The flow rate of air was fixed at $30 \mathrm{sccm}$. Activation energies were calculated from plots of $\ln (\sigma \mathrm{T}) \mathrm{vs} .1 / \mathrm{T}$.

\section{Results and Discussion}

Figure 4.2.13 shows the impedance spectra for 8 Dy4WSB at (a) $200{ }^{\circ} \mathrm{C}$, (b) $300{ }^{\circ} \mathrm{C}$ and (c) 500 ${ }^{\circ} \mathrm{C}$. The conductivities were calculated from the values of the bulk resistance measured at the frequency corresponding to the appropriate minima in the complex plots. Both Fig. 4.2.13 (a) and Fig. 4.2.13 (b) show the typical bulk impedance consisting of a single semicircle and a Warberg-type impedance indicating gas diffusion at the electrode. As the temperature increased (Fig. 2 (c)), the high frequency semicircle, attributable to the bulk resistance could no longer be resolved. The bulk resistance values were then calculated from the high frequency intercept.

The bulk conductivity results are plotted as $\log \sigma$ vs. 1000/T in Fig. 4.2.14. The compositions having $11-13 \%$ dopant (10Dy3WSB, 8Dy4WSB and 6Dy5WSB) showed higher conductivity than $17 \%$ dopant level (12Dy5WSB, 9Dy8WSB and 5Dy12WSB). A comparison of the conductivity of DyWSB and 20ESB is also shown in Fig. 4.2.14. 20ESB is considered in the literature to have the highest conductivity among the stabilized $\delta-\mathrm{Bi}_{2} \mathrm{O}_{3}$ 's, with conductivity at $800{ }^{\circ} \mathrm{C}, 500{ }^{\circ} \mathrm{C}$ and $200{ }^{\circ} \mathrm{C}$ of $0.41,0.021$ and $2.8 \times 10^{-5} \mathrm{~S} / \mathrm{cm}$, respectively. The conductivities of $8 \mathrm{Dy} 4 \mathrm{WSB}$, the highest 

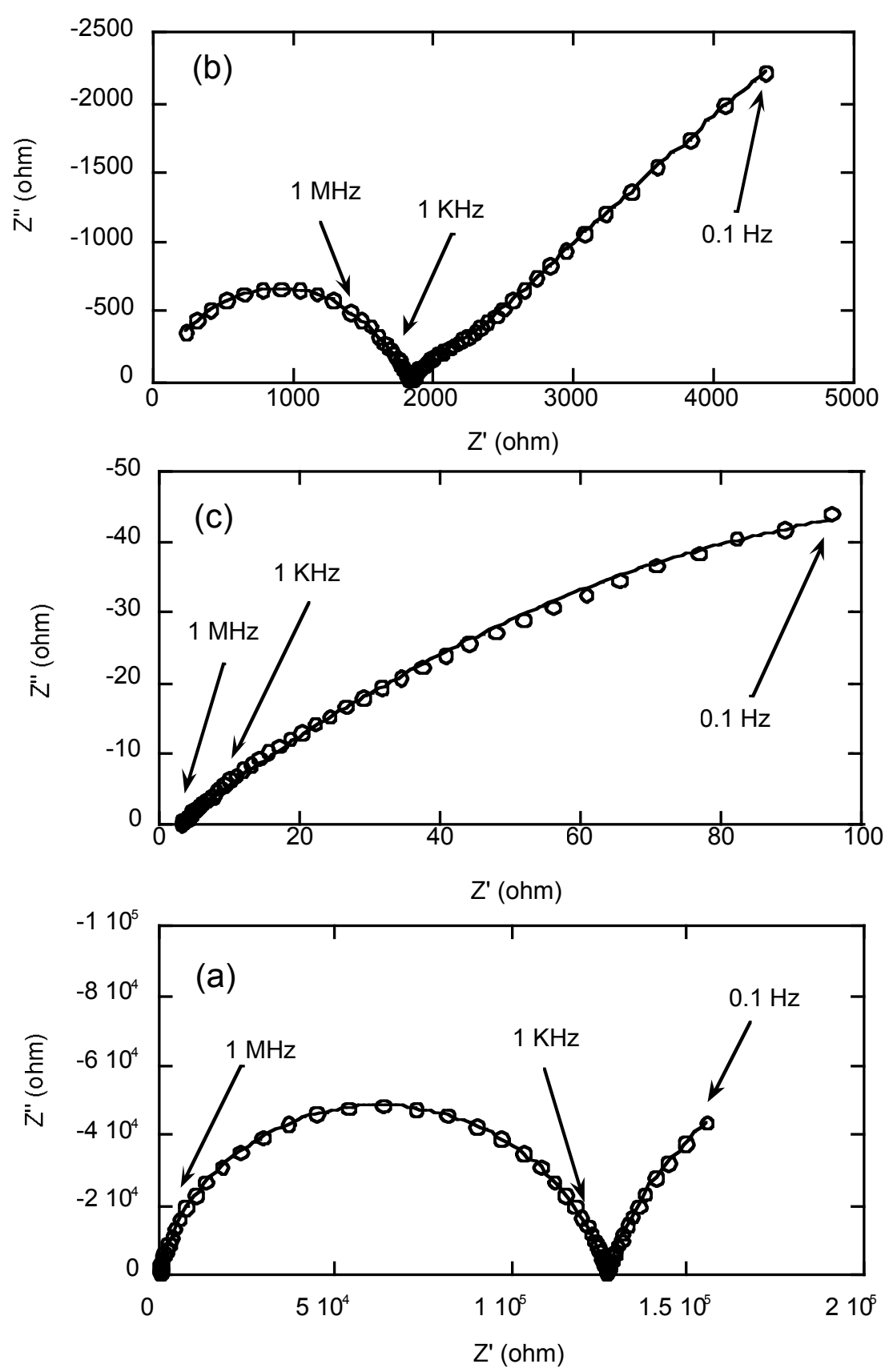

Figure 4.2.13. Impedance spectra of $8 \mathrm{Dy} 4 \mathrm{WSB}$ in air at (a) $200^{\circ} \mathrm{C}$ (b) $300^{\circ} \mathrm{C}$ and (c) $500^{\circ} \mathrm{C}$.

conductivity composition, at $800{ }^{\circ} \mathrm{C}, 500{ }^{\circ} \mathrm{C}$ and $200{ }^{\circ} \mathrm{C}$ are $0.56,0.043$ and $1.4 \times 10^{-4} \mathrm{~S} / \mathrm{cm}$, respectively, significantly higher than 20ESB, especially in the lower temperature region.

A change in activation energy for some of the compositions was observed, with a lower activation energy above $\sim 600^{\circ} \mathrm{C}$ and a higher activation energy below $\sim 600^{\circ} \mathrm{C}$. As the dopant concentration increases, the disparity between these two activation energies decreases. The observed disparity at $\sim 600^{\circ} \mathrm{C}$ as a function of dopant concentration is a general trend in stabilized bismuth oxide 


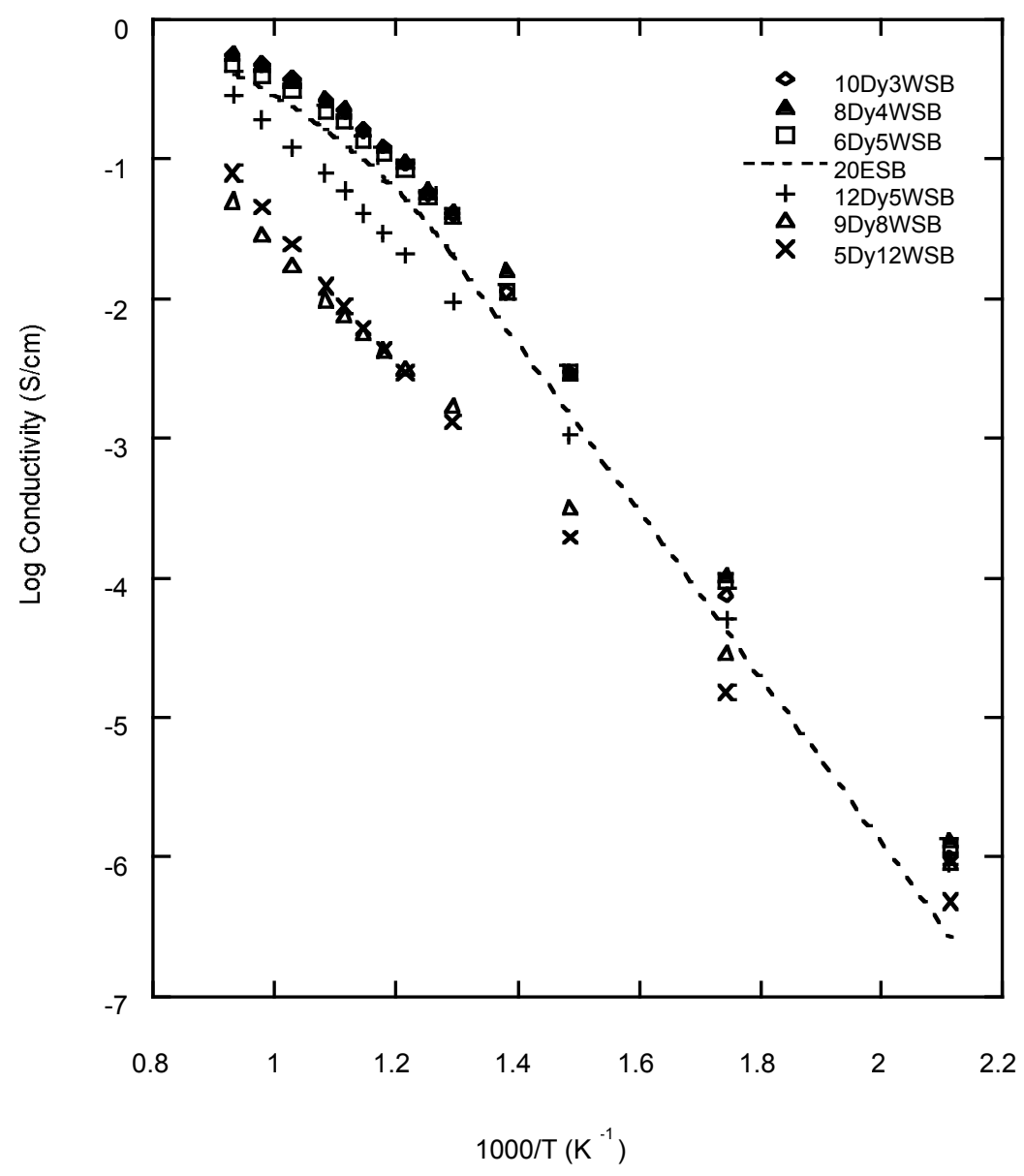

Figure 4.2.14. Arrhenius plot of conductivities for 10Dy3WSB, 8Dy4WSB, 6Dy5WSB, 12Dy5WSB, 9Dy8WSB, 5Dy12WSB and 20ESB

systems [57]. The activation energy values measured for low and high temperatures with different compositions are shown in Tables 4.2-11 and 4.2-12. From Table 4.2-11 and Fig. 4.2.14, it is clear that the lower dopant content compounds (11 - $13 \%$ dopant) showed two different conductivity activation energies. Conversely, the conductivity of $17 \%$ dopant level compositions (Table 4.2-12 and Fig. 4.2.14) showed single activation energies, except for 12Dy5WSB that exhibited a slight change in activation energy. This change in activation energy is attributed to an order-disorder transition of the

Table 4.2-11. Conductivity activation energies of $11-13 \%$ total dopant compositions

\begin{tabular}{ccc} 
Composition & Low Temperature $\left(<600^{\circ} \mathrm{C}\right) \mathrm{E}_{\mathrm{A}}(\mathrm{eV})$ & High Temperature $\left(>600^{\circ} \mathrm{C}\right) \mathrm{E}_{\mathrm{A}}(\mathrm{eV})$ \\
\hline 6Dy5WSB & 0.47 & 0.23 \\
8Dy4WSB & 0.47 & 0.29 \\
10Dy3WSB & 0.48 & 0.19 \\
\hline
\end{tabular}


oxygen sublattice $[59,64]$. Those compositions with the greatest disparity have the greatest tendency to order at low temperature $[57,68]$.

In Fig. 4.2.15, the conductivity in the $\mathrm{WO}_{3}-\mathrm{Dy}_{2} \mathrm{O}_{3}-\mathrm{Bi}_{2} \mathrm{O}_{3}$ system is plotted against the dopant composition ratio $\left(\mathrm{X}_{\mathrm{Dy}} /\left(\mathrm{X}_{\mathrm{Dy}}+\mathrm{X}_{\mathrm{W}}\right)\right)$ content at 500 and $700^{\circ} \mathrm{C}$. Values from the literature were used for comparisons at either end of the dopant composition ratio range $\left(\mathrm{WO}_{3}\right.$ stabilized $\mathrm{Bi}_{2} \mathrm{O}_{3}$ and $\mathrm{Dy}_{2} \mathrm{O}_{3}$ stabilized $\left.\mathrm{Bi}_{2} \mathrm{O}_{3}\right)$. 12WSB exhibits the highest conductivity among the phase stable $\mathrm{WO}_{3}$ compositions [63]. Thermal hysteresis in conductivity was observed at high temperature $\left(650{ }^{\circ} \mathrm{C}\right.$ to $\left.750{ }^{\circ} \mathrm{C}\right)$ for samples containing less than $20 \mathrm{~mol} \% \mathrm{WO}_{3}\left(\left(\mathrm{BiO}_{1.5}\right)_{0.89}\left(\mathrm{WO}_{3}\right)_{0.11}\right)$ due to the transformation from the monoclinic to the cubic structure. Therefore, the conductivity of 12WSB from Takahashi et al [63] is plotted (closed square) in Fig. 4.2.15.

Verkerk and Burggraaf [64] investigated DySB, in the composition range 5 to $60 \mathrm{~mol} \% \mathrm{Dy}_{2} \mathrm{O}_{3}$. The cubic phase for DySB containing 28.5 to $50 \mathrm{~mol} \% \mathrm{Dy}_{2} \mathrm{O}_{3}$ was found to be stable over the range of temperatures $\left(300{ }^{\circ} \mathrm{C}\right.$ to $\left.800{ }^{\circ} \mathrm{C}\right)$ used in the measurements. The samples with lower $\mathrm{Dy}_{2} \mathrm{O}_{3}$ content showed a phase transition from cubic to rhombohedral below $800{ }^{\circ} \mathrm{C}$ and no conductivity data was available. Therefore, values of 28.5DySB are plotted (closed triangle) on the right axis in Fig. 4.2.15 to compare with the doubly doped system, rather than comparing with the concentration range where polyphasic regions appear.

One of the effects of double doping $\mathrm{W}$ and Dy is that the cubic structure is stabilized at dopant concentrations less than that necessary for Dy alone. Thus, the cubic structure was stabilized at as little as $11-13 \%$ dopant concentration. At the higher total dopant concentration (17\%), intermediate between the dopant concentration of end compositions from the literature, there appears to be a minimum in conductivity at $\mathrm{X}_{\mathrm{Dy}} /\left(\mathrm{X}_{\mathrm{Dy}}+\mathrm{X}_{\mathrm{W}}\right)=0.4-0.5$ depending on temperature. In contrast, at the lower total dopant concentration $(11-13 \%)$, more closely matching the dopant concentration of the W end composition, there appears to be a maximum in conductivity at $\mathrm{X}_{\mathrm{Dy}} /\left(\mathrm{X}_{\mathrm{Dy}}+\mathrm{X}_{\mathrm{W}}\right)=0.5-0.6$,

Table 4.2-12. Conductivity activation energies of $17 \%$ total dopant compositions

\begin{tabular}{ccc}
\hline Composition & Low Temperature $\left(<600^{\circ} \mathrm{C}\right) \mathrm{E}_{\mathrm{A}}(\mathrm{eV})$ & High Temperature $\left(>600^{\circ} \mathrm{C}\right) \mathrm{E}_{\mathrm{A}}(\mathrm{eV})$ \\
\hline 5Dy12WSB & 0.39 & 0.39 \\
9Dy8WSB & 0.34 & 0.34 \\
12Dy5WSB & 0.42 & 0.34 \\
\hline
\end{tabular}




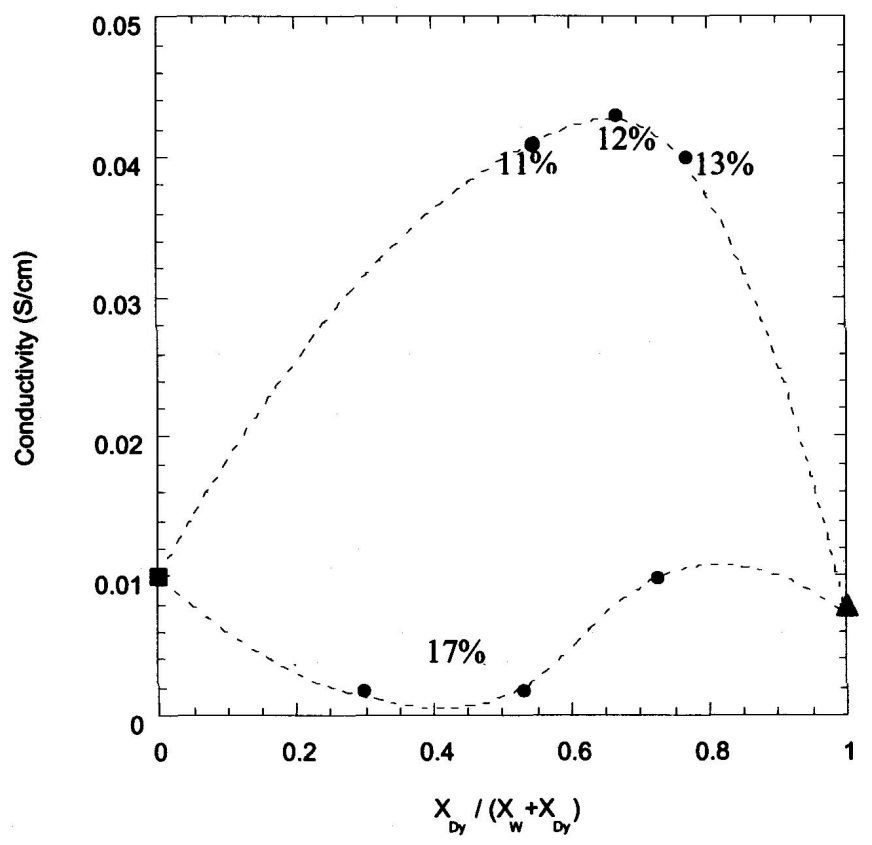

(a)

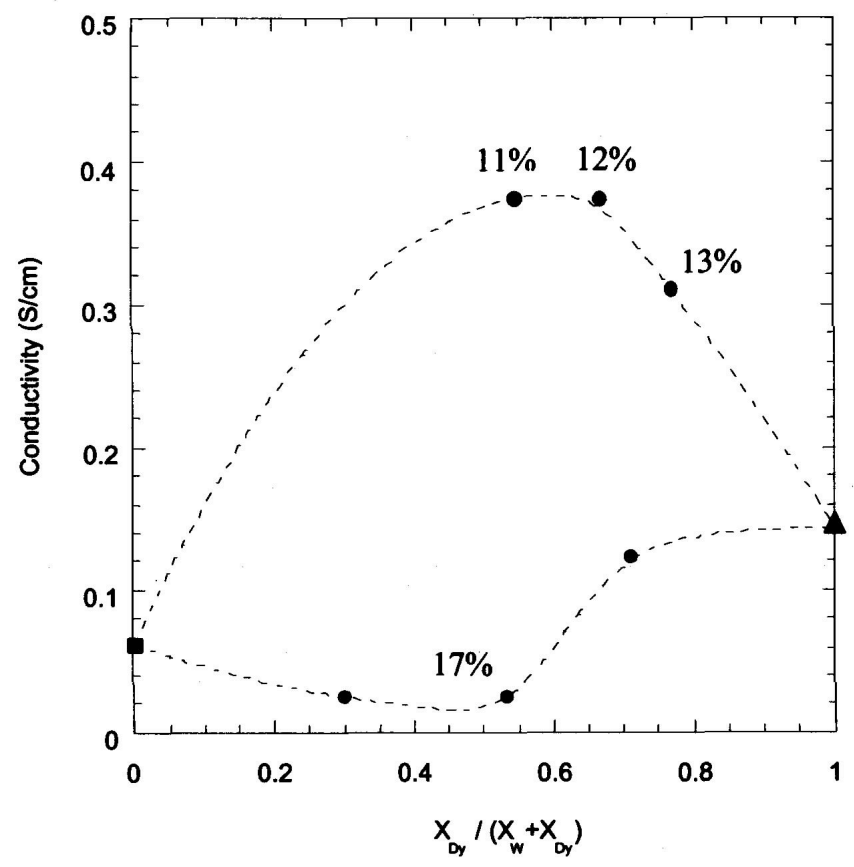

(b)

Figure 4.2.15. Conductivity as a function of dopant composition ratio $\left(\mathrm{X}_{\mathrm{Dy}} /\left(\mathrm{X}_{\mathrm{Dy}}+\mathrm{X}_{\mathrm{w}}\right)\right)$ and total dopant concentration at (a) $500^{\circ} \mathrm{C}$ and (b) $700^{\circ} \mathrm{C}$. The conductivity of $12 \mathrm{WSB}$ (Takahashi et al [63], closed square) and 28.5DySB (Verkerk et al [64], closed triangle) is given as a reference at either end of the range. 
depending on temperature. These results indicate the nature of the dopant (W vs. Dy) is as important as the total dopant concentration and that the effect of double doping on conductivity is "non ideal." The observed differences in conductivity dependence on dopant ratio warrants further investigation. Further discussion of phase and stability in the system is given in [68].

\section{Conclusion}

The conductivity of $\mathrm{Dy}_{2} \mathrm{O}_{3}$ and $\mathrm{WO}_{3}$ stabilized $\mathrm{Bi}_{2} \mathrm{O}_{3}$ was studied. These cations were selected for their high polarizability in order to achieve a stable disordered anion lattice and a high mobility. By double doping we were able to obtain compositions (11 - $17 \%$ dopant) that were cubic, when single dopants would not stabilize the cubic structure at this low of a dopant level. The resultant cubic DyWSB's had high conductivity. In particular, the composition 8Dy4WSB had conductivity significantly greater than 20ESB, rating it the most conductive oxide electrolyte currently known.

\subsubsection{Structural Stability and Conductivity of Cubic $\left(\mathrm{WO}_{3}\right)_{x}-\left(\mathrm{Dy}_{2} \mathrm{O}_{3}\right)_{y^{-}}-\left(\mathrm{Bi}_{2} \mathrm{O}_{3}\right)_{1-x-y}$}

The activation energy values measured for low and high temperatures with different compositions are shown in Tables 4.2-11 and 4.2-12. The $11-13 \%$ dopant compositions exhibited the disparity between high-temperature and low-temperature activation energies, which is a general feature of cubic stabilized bismuth oxides at low to moderate $(<30 \mathrm{~mol} \%)$ dopant concentration [69 82]. In contrast, the compositions containing a $17 \%$ dopant level showed no change in activation energy, even at this relatively low dopant level except for 12Dy5WSB that exhibited a slight change in activation energy. Those compositions with the greatest disparity have the greatest tendency to order at low temperature $\left(\sim 500^{\circ} \mathrm{C}\right)[74]$. 8Dy4WSB has a large discrepancy in activation energy and, as expected, in Fig. 4.2.16 shows a rapid decay in conductivity at $500{ }^{\circ} \mathrm{C}$.

The conductivity as a function of time at $500{ }^{\circ} \mathrm{C}$ for different dopant ratios with $17 \%$ dopant is shown in Fig. 4.2.17. As the ratio of W/Dy increased the initial (at $t=0$ ) conductivity decreased. However, the conductivity as a function of time became more stable. Among these, 12Dy5WSB showed a slight change in activation energy and have the highest conductivity and the highest aging rate. 9Dy8WSB and 5Dy12WSB did not exhibit a change in activation energy (Table 4.2-12) and showed negligible decay in conductivity as a function of time (Fig. 4.2.17). These results are consistent with the general trend of aging in stabilized bismuth oxides. The greater the disparity in activation energy, the greater was the aging rate. 


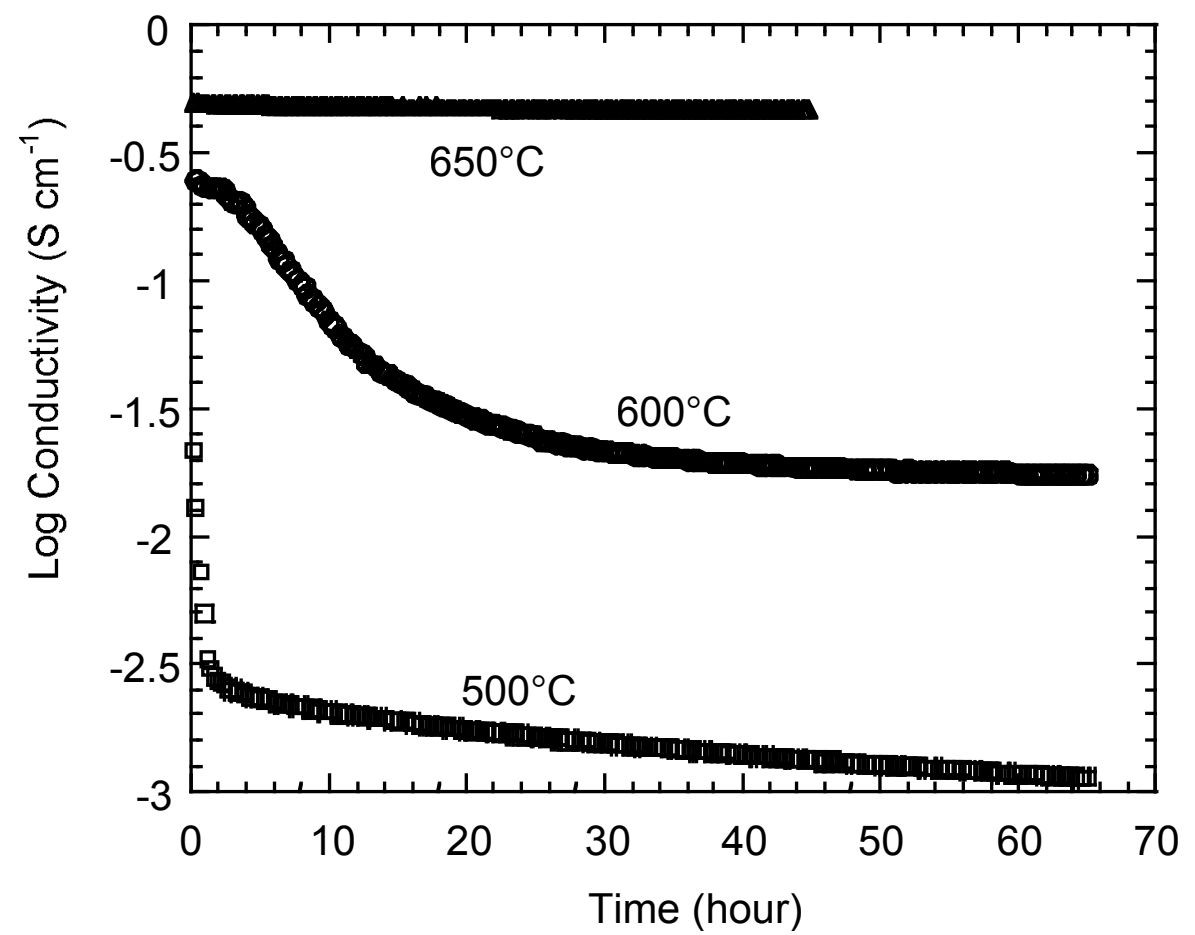

Figure 4.2.16. Conductivity vs. time for $8 \mathrm{Dy} 4 \mathrm{WSB}$ sample annealed at $650^{\circ} \mathrm{C}, 600^{\circ} \mathrm{C}$ and $500^{\circ} \mathrm{C}$

Aging and the resultant structural change at low temperature $\left(\leq 600^{\circ} \mathrm{C}\right)$ are independent of the fcc-rhombohedral phase transformation above $600^{\circ} \mathrm{C}$. Wachsman et al [74] discussed the critical issue involving the distinction between these two phenomena and showed that aging is a thermally reversible process, upon heating above the order-disorder transition temperature $\left(\sim 600^{\circ} \mathrm{C}\right)$. The ordered

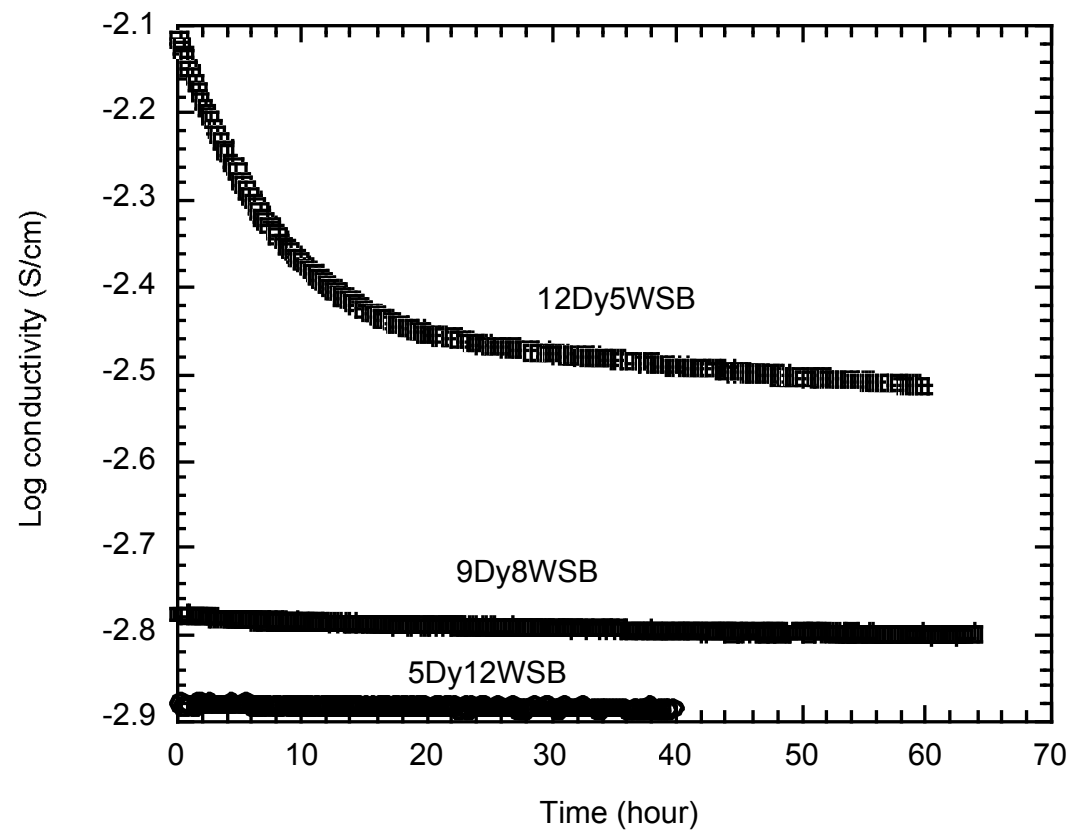

Figure 4.2.17. Conductivity vs. time for $12 \mathrm{Dy} 5 \mathrm{WSB}, 9 \mathrm{Dy} 8 \mathrm{WSB}$ and $5 \mathrm{Dy} 12 \mathrm{WSB}$ annealed at $500{ }^{\circ} \mathrm{C}$ 


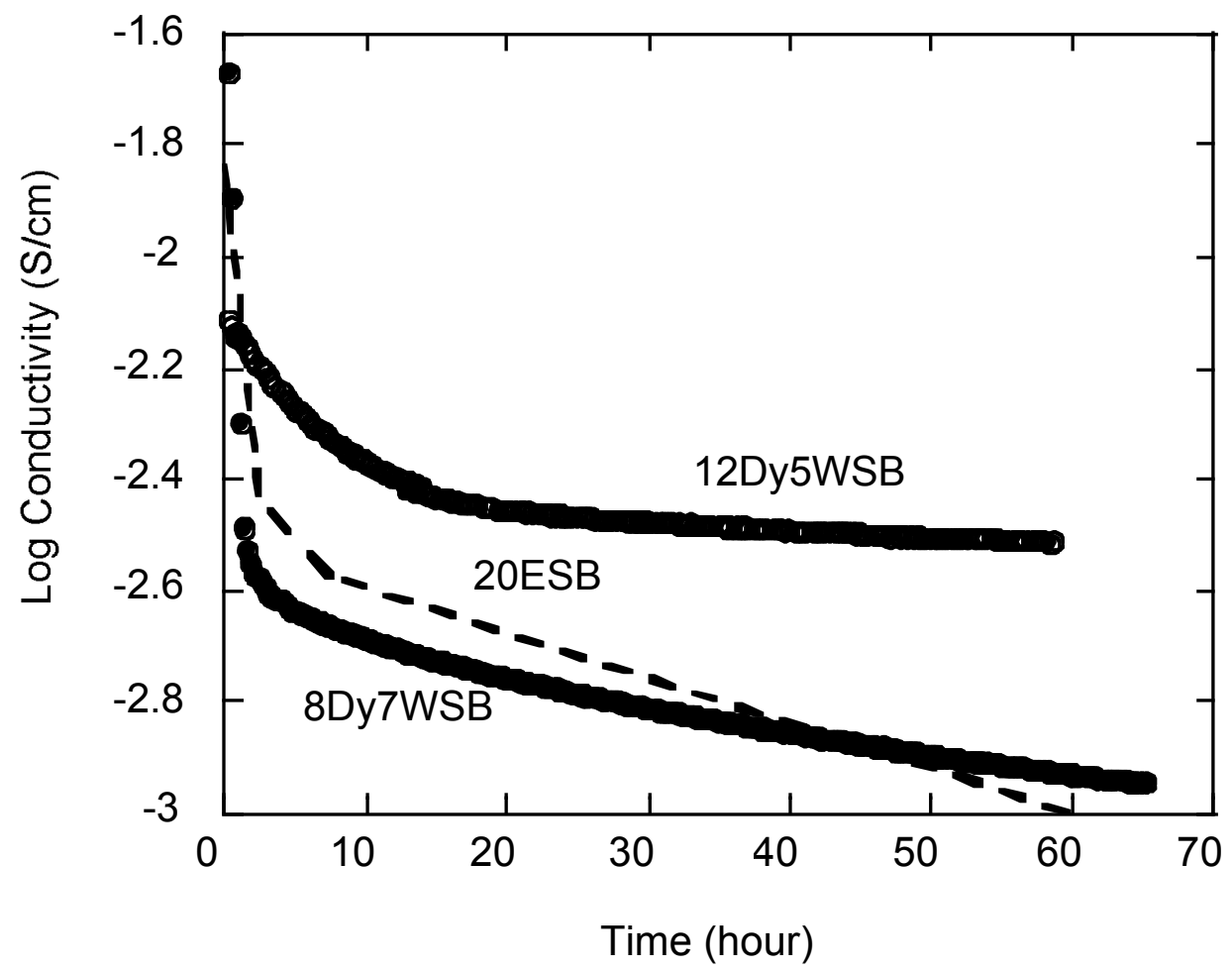

Figure 4.2.18. Conductivity vs. time for $12 \mathrm{Dy} 5 \mathrm{WSB}, 20 \mathrm{ESB}$ and $8 \mathrm{Dy} 4 \mathrm{WSB}$ samples annealed at $500^{\circ} \mathrm{C}$.

structure was relaxed at the temperature at which the fcc-rhombohedral phase transformation is most rapid $\left(\sim 650^{\circ} \mathrm{C}\right)$. As shown in Fig. 4.2.16, the conductivity of 8Dy4WSB aged at $650{ }^{\circ} \mathrm{C}$ did not change, indicating phase stability since the phase transformation occurs above the order-disorder transition temperature (between $600{ }^{\circ} \mathrm{C}$ and $720^{\circ} \mathrm{C}$ ) and results in conductivity degradation. However, the conductivity of the sample decayed at $600{ }^{\circ} \mathrm{C}$, the order-disorder transition temperature, and dropped rapidly at $500{ }^{\circ} \mathrm{C}$. This indicates that the decay is due to the ordering of the oxygen sublattice, rather than a conventional crystallographic transformation.

Figure 4.2.18 shows comparisons of 12Dy5WSB, 8Dy4WSB and 20ESB as a function of time. Both 20ESB and 8Dy4WSB showed a rapid drop in conductivity at $500^{\circ} \mathrm{C}$ within a few hours from their initial values of 0.021 and 0.043 to 0.00095 and $0.0012 \mathrm{~S} / \mathrm{cm}$, respectively, after 60 hours. Although the conductivity of 12Dy5WSB decayed from 0.010 to $0.0031 \mathrm{~S} / \mathrm{cm}$ after 60 hours, the kinetics of the aging for 12Dy5WSB were not as rapid. Comparison of the initial conductivity and the conductivity after 60 hours annealed at $500^{\circ} \mathrm{C}$ for selected bismuth oxide compounds is listed in Table 4.2-13. The conductivity value $(\sigma(t=60))$ of $12 \mathrm{Dy} 5 \mathrm{WSB}$ is greater than that of either ESB or $8 \mathrm{Dy} 4 \mathrm{WSB}$, and the value is greater than the initial values of 9Dy8WSB and 5Dy12WSB as well. 
Table 4.2-13. Comparison of conductivity values in selected stabilized bismuth oxides at $500^{\circ} \mathrm{C}$, where $\sigma(t=0)$ is the initial conductivity and $\sigma(t=60)$ is the conductivity at 60 hours.

\begin{tabular}{lccc}
\hline \multicolumn{1}{c}{ Composition } & $\begin{array}{c}\text { Conductivity }(\mathrm{S} / \mathrm{cm}) \\
t=0\end{array}$ & $\begin{array}{c}\text { Conductivity }(\mathrm{S} / \mathrm{cm}) \\
t=60 \mathrm{hr}\end{array}$ & Reference \\
\hline$*\left(\mathrm{BiO}_{1.5}\right)_{0.8}\left(\mathrm{ErO}_{1.5}\right)_{0.2}$ & 0.021 & 0.00095 & {$[74]$} \\
$\left(\mathrm{BiO}_{1.5}\right)_{0.88}\left(\mathrm{DyO}_{1.5}\right)_{0.08}\left(\mathrm{WO}_{3}\right)_{0.04}$ & 0.043 & 0.0012 & this work \\
$\left(\mathrm{BiO}_{1.5}\right)_{0.83}\left(\mathrm{DyO}_{1.5}\right)_{0.12}\left(\mathrm{WO}_{3}\right)_{0.05}$ & 0.010 & 0.0031 & this work \\
$\left(\mathrm{BiO}_{1.5}\right)_{0.83}\left(\mathrm{DyO}_{1.5}\right)_{0.09}\left(\mathrm{WO}_{3}\right)_{0.08}$ & 0.0017 & 0.0016 & this work \\
$\left(\mathrm{BiO}_{1.5}\right)_{0.83}\left(\mathrm{DyO}_{1.5}\right)_{0.05}\left(\mathrm{WO}_{3}\right)_{0.12}$ & 0.0014 & 0.0013 & this work \\
$*\left(\mathrm{BiO}_{1.5}\right)_{0.715}\left(\mathrm{DyO}_{1.5}\right)_{0.285}$ & 0.0071 & & {$[82]$} \\
$*\left(\mathrm{BiO}_{1.5}\right)_{0.88}\left(\mathrm{WO}_{3}\right)_{0.12}$ & 0.010 & & {$[81]$} \\
\hline
\end{tabular}

* Highest conductivity compositions that exhibit fcc structure were selected.

Values from the literature for the highest conductivity compositions of singly Dy and $\mathrm{W}$ doped $\mathrm{Bi}_{2} \mathrm{O}_{3}$ were used for comparisons, but no aging data of 28.5DySB and 22WSB were available in the literature. Their initial values are comparable to that of 12Dy5WSB. However, the singly doped bismuth oxides exhibit polyphasic regions in the dopant concentration range and are stable only over a narrow composition range. As compared to 20ESB (Fig. 4.2.18) the more stable conductivity of 12Dy5WSB is a major improvement for low temperature $\left(<600{ }^{\circ} \mathrm{C}\right)$ applications. For high temperature $\left(>600{ }^{\circ} \mathrm{C}\right)$ applications, 8 Dy4WSB is phase stable, does not order, and has the highest conductivity (Fig. 4.2.16).

\section{Conclusion}

The electrical conductivity of Dy-W "stabilized" bismuth oxides (DyWSB) was studied. 20ESB has been known to have the highest conductivity among all the oxide electrolytes. However, this oxide exhibits a degradation of conductivity below $600{ }^{\circ} \mathrm{C}$. 8Dy4WSB demonstrates a conductivity greater than that of 20ESB, but it shows rapid aging at $500{ }^{\circ} \mathrm{C}$. Among the compositions of DyWSB studied, 8 Dy4WSB is stable above $600{ }^{\circ} \mathrm{C}$ and has the highest conductivity of any known electrolyte. Below $600{ }^{\circ} \mathrm{C} 12 \mathrm{Dy} 5 \mathrm{WSB}$ shows great possibility to improve stability of the disordered lattice at low temperature, while maintaining high conductivity. However, more experiments on other compositions in the $\mathrm{Dy}-\mathrm{W}-\mathrm{Bi}_{2} \mathrm{O}_{3}$ system may result in obtaining even higher and more stable conductivity.

\subsubsection{Role of Dopants in Other Fluorite Systems}

It is well known that the conductivity of oxides such as $\mathrm{ZrO}_{2}$ stabilized in the fluorite structure by lower valent dopants (e.g., $\mathrm{Y}_{2} \mathrm{O}_{3}$ and $\mathrm{CaO}$ ) increases with increasing dopant concentration in the dilute (low dopant concentration) regime. This increase in conductivity is due to the increase in 
concentration of charge compensating anion vacancies $\left(\mathrm{Vo}^{*}\right)$, the conducting species in these materials. It is also well known that the conductivity reaches a maximum at about 8-10 mole\%, depending on the dopant. The ensuing decrease in conductivity with increasing dopant concentration is due to ordering of the anion sublattice. This ordering occurs in part because the concentration of $\mathrm{Vo}{ }^{\circ}$ is no longer in the dilute limit and $\mathrm{Vo} \cdot{ }^{-}-\mathrm{Vo} \cdot$ interactions have a higher statistical probability. Further, dopant-vacancy interactions (e.g., $\mathrm{Y}_{\mathrm{Zr}}{ }^{\prime}-\mathrm{Vo}^{\circ}$ ) also have a higher probability of occurrence.

For some time, the conventional wisdom was that for yttria stabilized zirconia (YSZ), increasing $\mathrm{Y}_{\mathrm{Zr}}{ }^{\prime}$ concentration resulted in associated defects $\left(\mathrm{Y}_{\mathrm{Zr}}{ }^{\prime}-\mathrm{Vo}{ }^{\circ}\right)^{\bullet}$ at low $\mathrm{Y}_{\mathrm{Zr}}$ ' concentration and $\left(\mathrm{Y}_{\mathrm{Zr}}{ }^{\prime}-\mathrm{Vo}^{\circ} \cdot\right.$ $\left.\mathrm{Y}_{\mathrm{Zr}}{ }^{\prime}\right)^{\mathrm{x}}$ at higher $\mathrm{Y}_{\mathrm{Zr}}$ ' concentration [83 - 85]. These dopant-vacancy associated defects were responsible for the decreasing conductivity with increasing dopant concentration and were due to the Coulombic attraction between the negatively charged dopant and positively charged anion vacancy. However, recent studies with high resolution X-ray and neutron techniques have indicated a more complex situation involving vacancy clustering and a dopant radii dependence on associated defects $[86-88]$.

With respect to the later point, the work of $\mathrm{Li}$ et al. [86] indicates that for $\mathrm{M}^{3+}$ dopant radii less than the host $\mathrm{Zr}^{4+}$ cation radii the oxygen vacancies associate with the dopant, consistent with expectations based on electrostatic considerations. However, for $\mathrm{M}^{3+}$ dopant radii greater than the host $\mathrm{Zr}^{4+}$ cation radii the oxygen vacancies associate with the host cation. For YSZ, since the radii of $\mathrm{Y}^{3+}$ is greater than $\mathrm{Zr}^{4+}$, the vacancy associates that are formed are $\left(\mathrm{Zr}_{\mathrm{Zr}}{ }^{\mathrm{x}}-\mathrm{Vo}^{\cdot}{ }^{*}-\mathrm{Zr}_{\mathrm{Zr}}\right)^{\mathrm{x}}{ }^{\cdot \bullet}$ and the $\mathrm{Y}^{3+}$ are 8 fold coordinated with $\mathrm{O}_{\mathrm{O}}{ }^{\mathrm{x}}$, in direct contrast to the expectation based on electrostatic considerations.

Therefore, there is a competition between electrostatic attraction, due to the difference in cation oxidation states, and structural considerations, due to the difference in cation radii, that determine whether the vacancy associates with the host or dopant cation. First principles calculations by Bogicevic et al. indicate that in this competition structural considerations dominate [89]. If for large radii dopants the vacancies associate with the host cations, then the decrease in conductivity with increasing dopant concentration is not due to dopant-vacancy associates and must be due to some form of ordering of the charge compensating vacancies at high vacancy concentrations.

Bogicevic et al.'s calculations show that vacancies repel each other but that they have a tendency to align as third nearest neighbors along $<111>$ directions [89]. Thus, increasing vacancy concentrations would tend to result in $<111>$ ordered arrays of oxygen vacancies. Similarly, Goff et 
al. has shown that vacancy clusters form and proposed that the decrease in conductivity with increasing vacancy concentration is due to the formation of "static aggregates" [87].

$\mathrm{Bi}_{2} \mathrm{O}_{3}$ stabilized in the fluorite structure by isovalent cations has an extremely high concentration of $\mathrm{Vo}^{*}(25 \%$ of the anion sites) and no electrostatic difference between host and dopant cations. As such, cubic bismuth oxides provide an excellent model of how anion transport in oxides with the fluorite structure is influenced by high vacancy concentration and structural considerations in the absence of Coulombic attraction.

\section{Ordered And Disordered Structures}

From the above studies we showed that the ordered structure consisted of both "occupational ordering" and "positional ordering". The occupational ordering can be described by alignment of vacant $8 \mathrm{c}$ anion sites in $<111>$ directions, resulting in the TEM observed $2 \mathrm{a} \times 2 \mathrm{a} \times 2$ a superstructure [90 - 94]. The alignment of anion vacancies along $<111>$ directions in the ordered structure of cubic $\mathrm{Bi}_{2} \mathrm{O}_{3}$ is consistent with the theoretical results for stabilized zirconia [89].

The positional ordering consisted of displacement of the occupied anion sites in a $<111>$ direction from the $8 \mathrm{c}$ to $32 \mathrm{f}$ sites, as observed by neutron diffraction [95 - 97]. Both of these types of ordering were required to obtain a structure factor consistent with experimental results. The resultant ordered structure is shown in Fig. 4.2.5. The disordered structure was similarly investigated, and can be described by a simple fluorite structure (single unit cell) where the anion sites are statistically occupied by 3/4 oxygen ion (no occupational ordering). The disordered structure does have, however, some positional ordering.

\section{Effect Of Structure On Conductivity}

Through the investigations of the ordered and disordered structures we determined the position and occupancy of each anion site in lanthanide "stabilized" cubic bismuth oxide. From this we calculated the dependence of jump distance $\left(\lambda=\left[0.866 \times 8 \mathrm{c}_{\text {occupancy }}+0.693 \times 32 \mathrm{f}_{\text {occupancy }}\right] \mathrm{a} / 3\right)$ on structure as well as available jump directions $(n=6$ for disordered and 1 for ordered structure), charge $(\mathrm{ze}=1.5 \mathrm{e}$ for disordered structure and $2 \mathrm{e}$ for ordered structure $)$ and concentration of mobile anions in a jump allowed position ( $c_{i}=1$ for disordered and 0.333 for ordered structure) [98 - 99]. With this information we calculated the conductivity pre-exponential factor (A) for the ordered and disordered structures. 
The decrease in jump distance due to positional ordering, and the decrease in available jump directions and concentration of mobile vacancies due to the combination of both types of ordering decreased the pre-exponential factor by an order of magnitude. These calculated results were in close agreement with experimental results. Further, these investigations showed that the decrease in preexponential factor overshadowed the effect of the decrease in activation energy upon ordering.

The influence of dopant radii and polarizability on structure dominated the pre-exponential factor and thus the conductivity, with larger more polarizable dopant cations providing a more stable disordered structure and resultant higher conductivity. To confirm this we synthesized a cubic $\mathrm{Bi}_{2} \mathrm{O}_{3}$ stabilized with two highly polarizable dopants, Dy and W. The resultant DWSB has a conductivity twice that of ESB making it the highest conductivity phase stabilized fluorite oxide [100 - 102].

\section{Conclusion}

There is a strong tendency for anions to order in $<111>$ in highly defective fluorite oxides (anion vacancy concentrations $>\sim 10 \%$ ). This has been reported for zirconia [ $86-89]$, and as discussed here for bismuth oxide, and would appear to be a common feature for all fluorite oxides.

All of the lanthanide $\mathrm{M}^{3+}$ dopants investigated have the same valence as $\mathrm{Bi}^{3+}$ and a radii less than $\mathrm{Bi}^{3+}$. Thus ordering of the anion lattice is due to differences in radii and polarizabilty, but not electrostatic differences between the host and dopant cations. As the radii increased toward that of $\mathrm{Bi}^{3+}$ the disordered structure was more stable. Thus, there was a lower tendency to form an ordered anion lattice and/or vacancy associates.

Conversely, the closer the dopant radii is to the host cation radii, the lower the tendency is to order the anion lattice. Ideally, then the dopant would have the same radii as the host. However, when the difference in radii is too small the fluorite structure cannot be stabilized down to room temperature [92].

Finally, $\mathrm{Bi}^{3+}$ is a highly polarizable cation due to its lone pair of electrons. As the radii of the lanthanide dopants was increased toward that of the $\mathrm{Bi}^{3+}$ host cation the polarizability of these dopants also increased toward that of the $\mathrm{Bi}^{3+}$ host cation. Thus, further investigation into the relative influence of radii vs. polarizability is warranted. 


\subsection{Synthesis and Characterization of GDC $\backslash E S B$ Electrolytes}

In this section, we describe the synthesis of bismuth oxide/ceria bilayer electrolyte - samariumdoped ceria (SDC: $\mathrm{Ce}_{0.8} \mathrm{Sm}_{0.2} \mathrm{O}_{1.9}$ ), gadolinium-doped ceria (GDC: $\mathrm{Ce}_{0.9} \mathrm{Gd}_{0.1} \mathrm{O}_{1.95}$ ), and erbiumstabilized bismuth oxide (ESB: $\mathrm{Bi}_{0.8} \mathrm{Er}_{0.2} \mathrm{O}_{1.9}$ ). The bilayer electrolyte precursors were synthesized by two processes solid-state synthesis and the citrate method. SDC $\ E S B$ electrolytes were characterized through the usual techniques (XRD, SEM, EDS), conductivity measurements and through electrochemical performance tests (OCP and I-V). To investigate the effect of relative thickness of bilayer SDC \ESB electrolytes on OCP and $t_{i}$, five cells were prepared by depositing ESB layers via PLD and dip-coating of varying thickness on identical SDC substrates.

\subsubsection{Thin Film Preparation}

Oxide thin films have been prepared by a variety of methods such as spray RF ion plating [103], laser spraying [104], spray pyrolysis [105], sputtering [106], chemical vapor deposition (CVD) [107], and pulsed laser deposition (PLD) [108]. PLD is suitable for preparing high-quality thin films of multi- component oxides. This technique requires temperature around $500{ }^{\circ} \mathrm{C}$ to $700{ }^{\circ} \mathrm{C}$. The bilayer electrolytes can also be produced from a slurry coating [109, 110]. One of the advantages of slurry coating over PLD is that up-scaling to component size and volume is much more practical. Dipcoating is one example of a slurry coating technique. This procedure is simple, has precise composition control, and is a low processing temperature technique. This study used both PLD and dip-coating techniques to fabricate ESB thin films on either SDC or GDC substrates.

\section{Pulsed-Laser Deposition (PLD)}

Figure 4.3.1 shows a typical PLD system. ESB thin films were deposited on one side of polished SDC substrates. Film thicknesses were measured by profilometry (Alpha-step 500, Tencor) around the outer edge of the samples where two small sample holders were placed to mask these areas from deposition. The frequency of the $\mathrm{KrF}$ excimer laser (Lambda Physik) was set at $10 \mathrm{~Hz}$, and the laser energy was $500 \mathrm{~mJ}$, yielding a substrate temperature of $700{ }^{\circ} \mathrm{C}$.

Figure 4.3.2 shows a linear relationship between film thickness and deposition time for the growth of an ESB film on an SDC substrate. A key feature of the ESB film is that it should be pinhole free to block access of the cathode (or cathodic conditions) to SDC. By so doing, the ESB layer prevents electronic conduction (which occurs in single layer SDC membranes) in the bilayered 


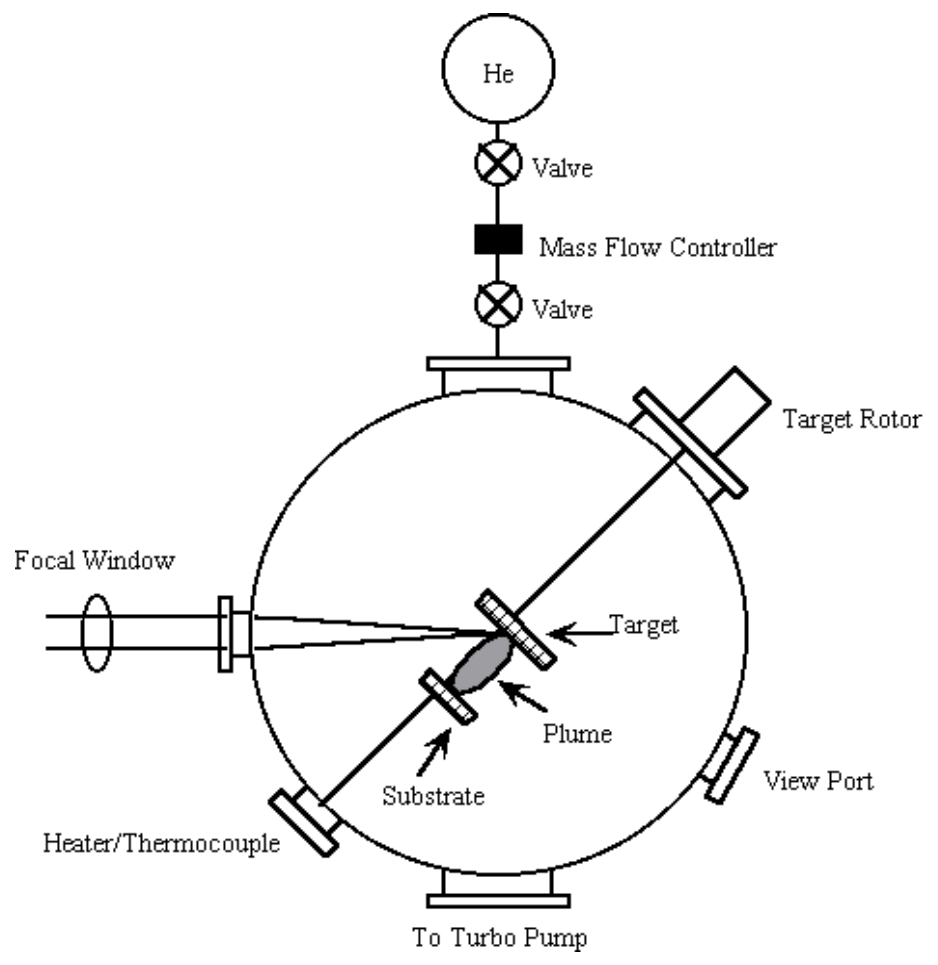

Figure 4.3.1. Experimental system of pulsed laser deposition (PLD) [111].

membrane. The thickness of the ESB film was limited to $2 \mu \mathrm{m}$. This limitation was imposed by the long depositions times required (it took several hours each to evacuate, heat up, deposit and cool down).

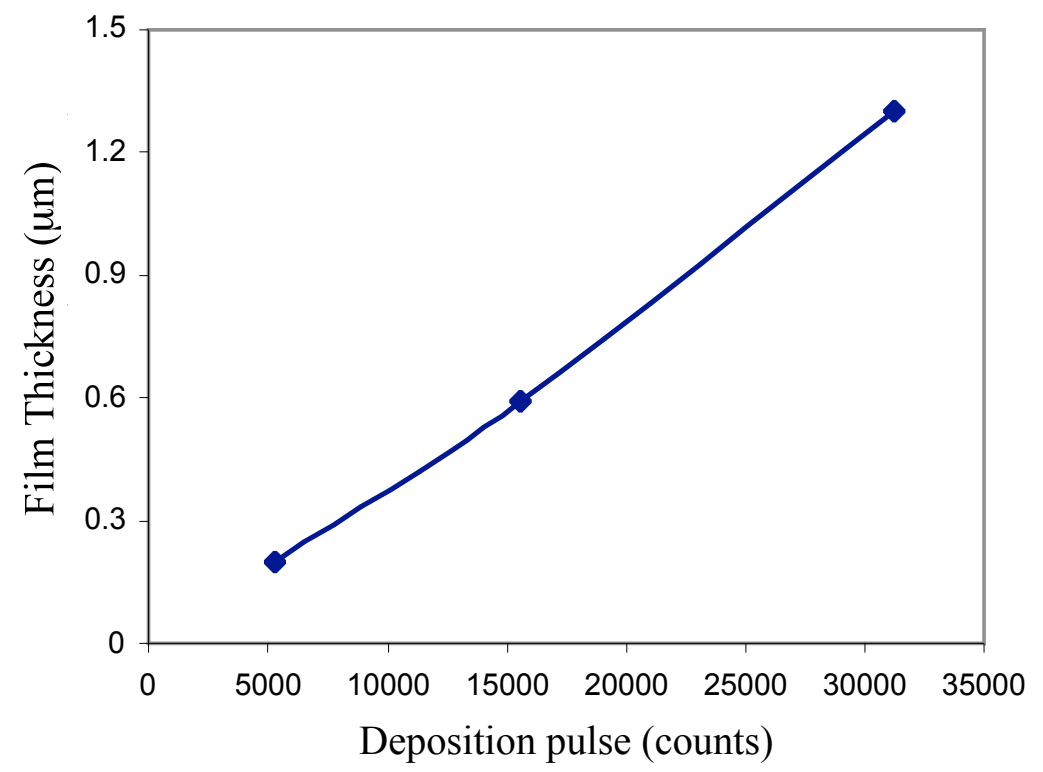

Figure 4.3.2. ESB film thickness on SDC substrate as a function of deposition time (pulses) 


\section{Colloidal Deposition: Dip-Coating.}

Bilayers were also fabricated via dip-coating. For this process, 5 wt. $\%, 7$ wt.\%, and 10 wt. $\%$ ESB slurries were prepared and dip-coated on one side of the substrate. The dipping cycle was repeated to obtain the desired ESB thicknesses. A detailed description of the dip-coating process is provided in section 4.4. The film-coated bilayer electrolytes were then sintered at $890{ }^{\circ} \mathrm{C}$ for $10 \mathrm{hrs}$. XRD, SEM, and EDS were performed to analyze quality of the film and to measure the film thickness.

\subsubsection{Characterization of Bilayer SDC|ESB and GDC|ESB Electrolytes}

\section{Pulse Laser Deposition (PLD).}

The XRD patterns in Fig. 4.3.3 show that a fluorite structure was obtained for SDC, ESB and the bilayered SDC $\backslash E S B$ samples. This result suggests that other phases (arising from reactions between SDC and ESB at their interface) are not present to any large degree and, by extension, that PLD is an acceptable way to deposit ESB on an SDC substrate.

Figure 4.3.4 shows an SEM micrograph of the cross-section of an ESB film, formed by PLD, on a dense SDC substrate, i.e., the SDC\ESB bilayer. Cross-sectional views indicate that the film adhered well to the baseline ceria substrate. The chemical composition of the bilayered SDC\ESB electrolytes was obtained, Fig. 4.3.5, from energy dispersive analysis (EDS). The SEM in Fig. 4.3.4(b) shows the region that was line-scanned and Fig. 4.3.5 shows the compositional results of the line-scan. It is apparent (at this resolution) in Fig. 4.3.5(b), that neither solid-state reaction nor inter-diffusion between

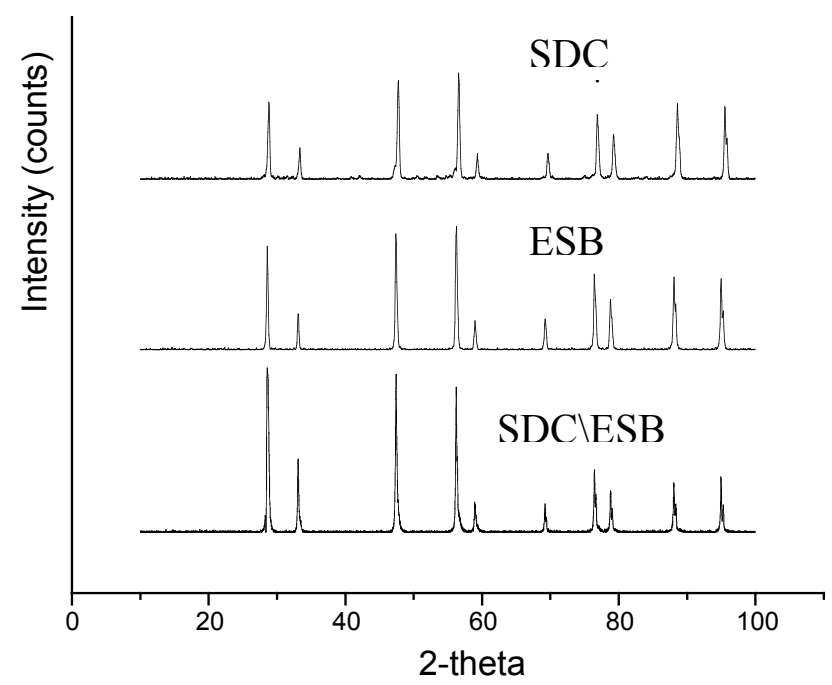

Figure 4.3.3. X-ray diffraction patterns of calcined SDC powder at $1300{ }^{\circ} \mathrm{C}$ for $10 \mathrm{hrs}$, calcined ESB powder at $800^{\circ} \mathrm{C}$ for $16 \mathrm{hrs}$, and SDC coated with ESB (ESB film thickness: $0.2 \mu \mathrm{m}$ ) via PLD. 

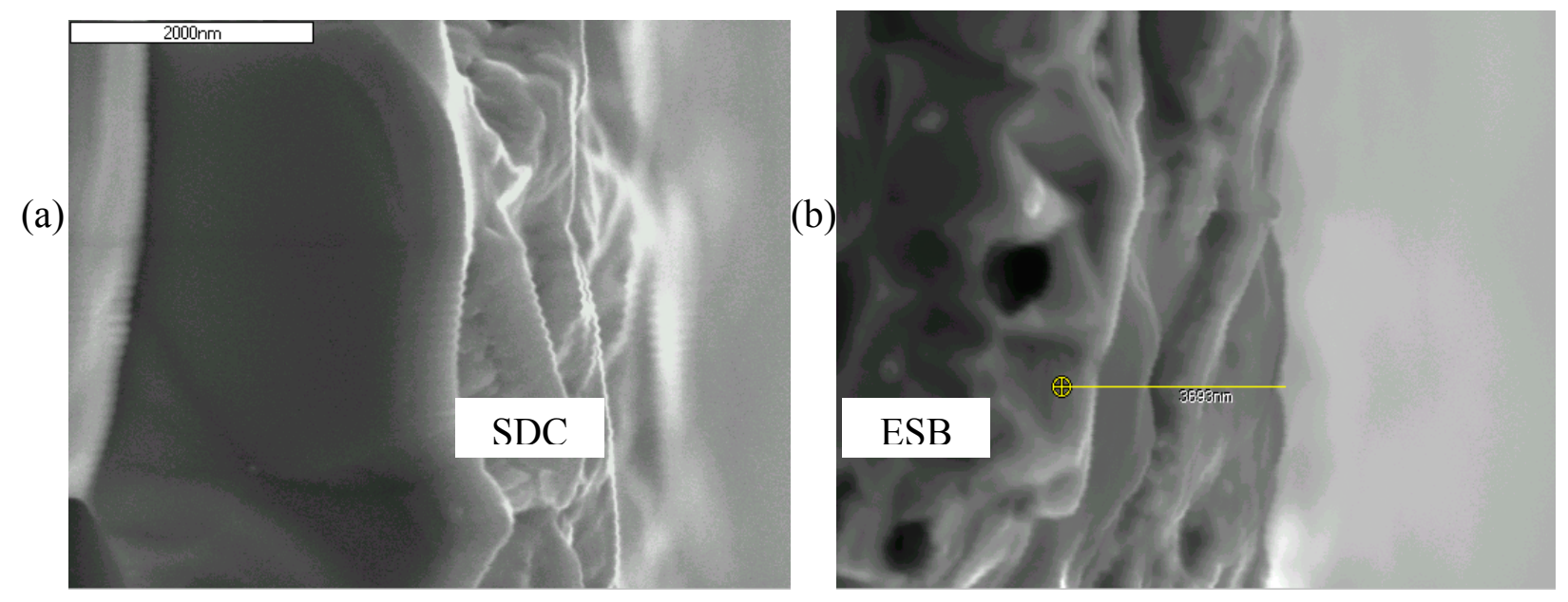

Figure 4.3.4. Cross-section SEM of ESB film on a dense SDC substrate (SDC $\backslash$ ESB bilayer), (b) shows line-scanned region

(b)
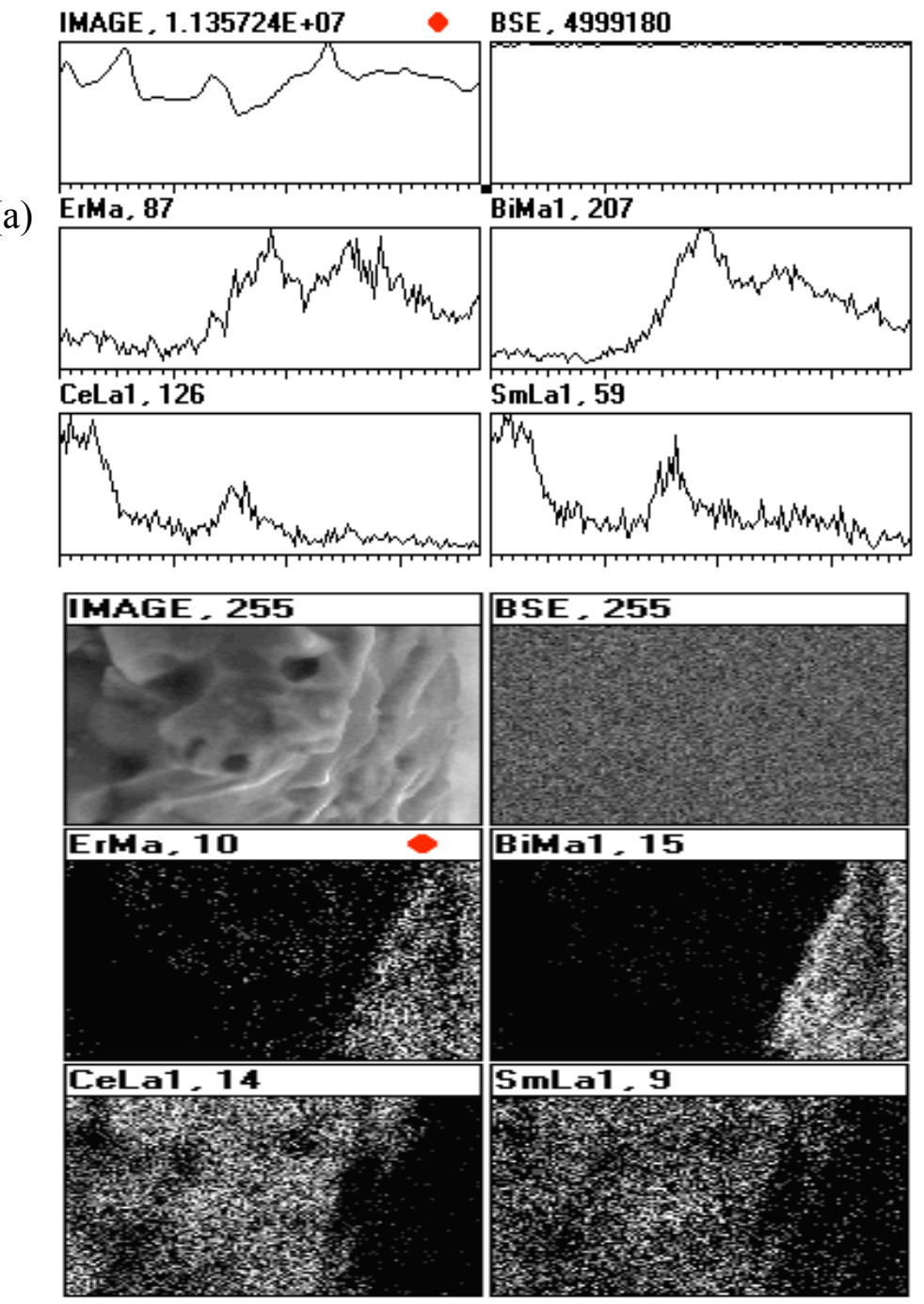

Figure 4.3.5. (a) X-ray line-scan and (b) X-ray map of elements in SDC $\backslash E S B$ sample 


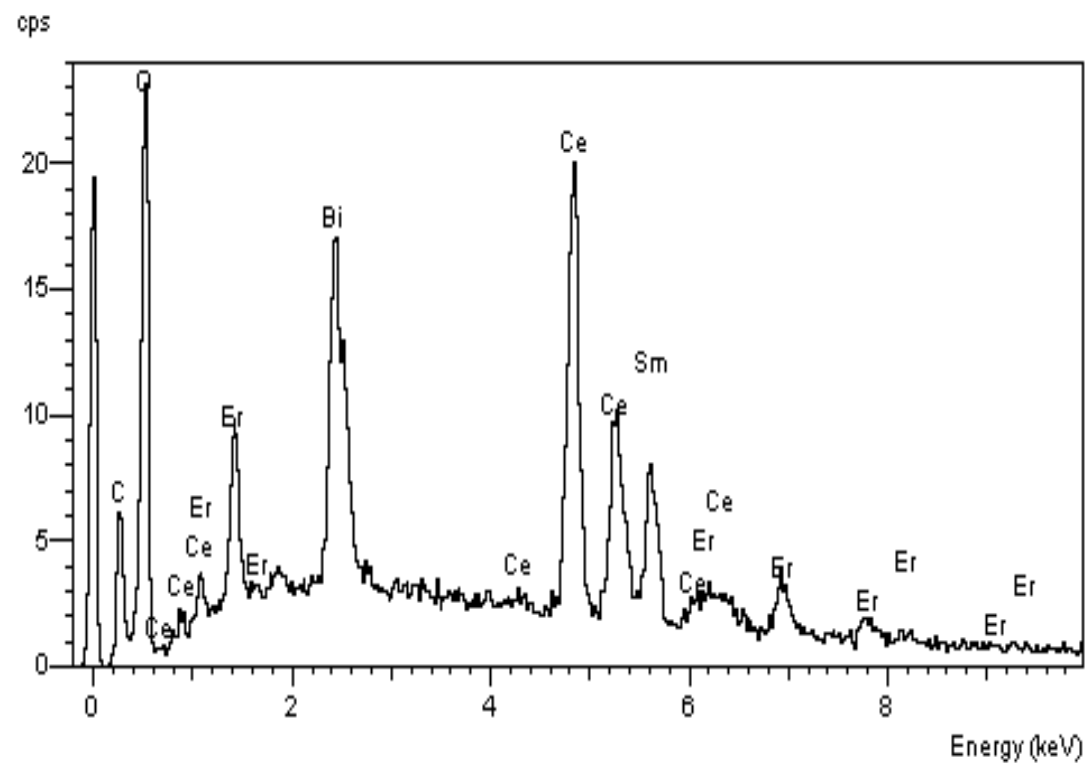

Figure 4.3.6. EDS spectra of the SDC $\backslash E S B$ sample cross-section.

the phases has occurred. Finally, in Fig. 4.3.6, the EDS spectra of the SDCIESB sample cross-section shows that any impurity levels are below detectable limits.

\section{Dip-coating}

Three different concentrations of the ESB gel were used for dip-coating, resulting in a final film thickness ranging from 9 to $30 \mu \mathrm{m}$, by SEM micrograph. Figure 4.3.7 is the cross-section SEM micrograph of the different ESB film on dense SDC substrate via dip-coating. It shows that the film adheres well to the baseline SDC substrate. However, both film thickness and densification increased with the amount of ESB in the sol. The film fabricated by $5 \mathrm{wt} . \%$ ESB gel exhibited a porous film surface, but with the $10 \mathrm{wt} . \%$ ESB gel a dense film surface was obtained. Concentrations of more than 10 wt.\% ESB did not form a stable gel for coating. The process needs further work to improve the stability of the gel (perhaps by introducing a dispersant). Finally, significant inter-diffusion between two phases was not detected from EDS, Fig. 4.3.8, of the samples.

\section{Conductivity}

The effective ${ }^{3}$ conductivities of SDC $\mid E S B$ sample (ESB film thickness: $\sim 0.2 \mu \mathrm{m}$ ) were measured in air by EIS. Figure 4.3.9 shows that the effective conductivity of the SDC $\backslash E S B$ bilayer was higher

\footnotetext{
${ }^{3}$ Conductivity is an intrinsic material property, independent of sample geometry. Hence a bilayer does not have a true conductivity. The term effective conductivity is used here to describe a hypothetical material with a conductivity that is equivalent to the bilayer.
} 
(a)

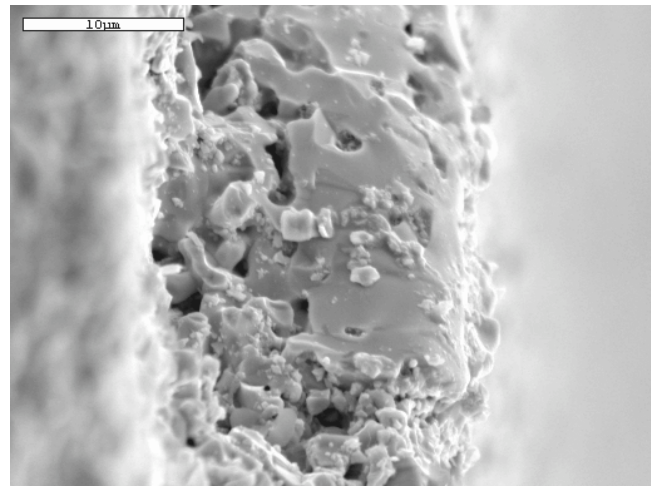

(b)

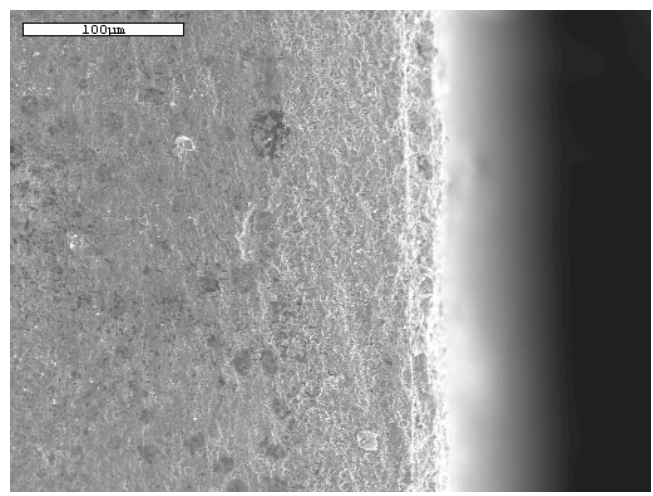

(c)

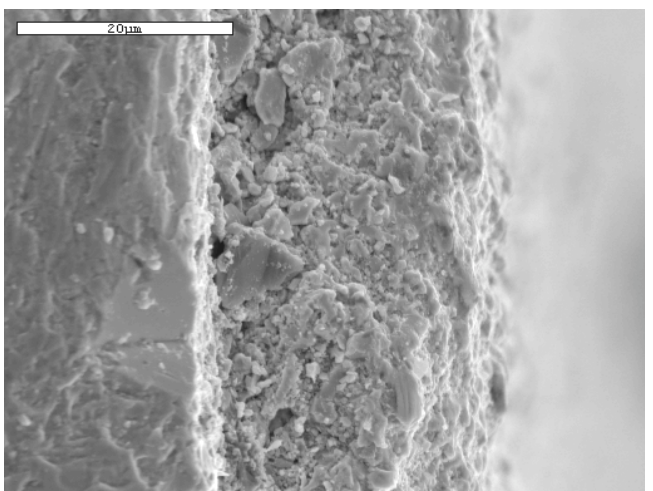

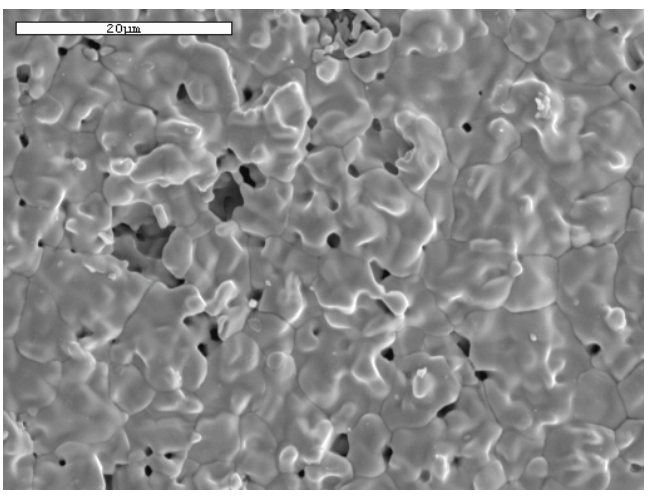
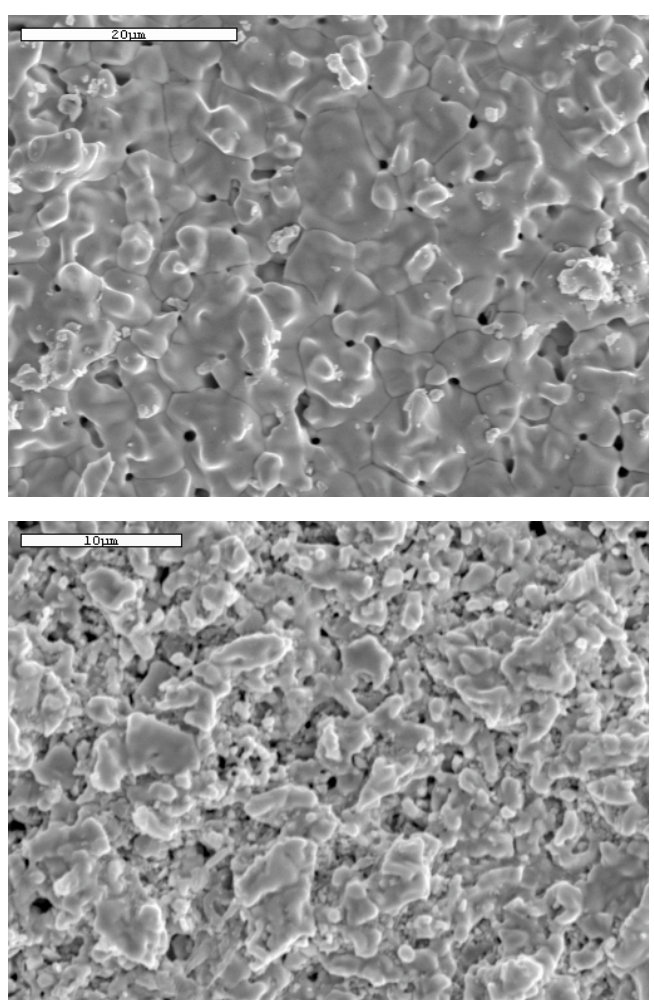

Figure 4.3.7. Cross-section SEM micrograph of the ESB film on dense SDC substrate via dip-coating: (a) 5 wt.\%, (b) 7wt.\%, and (c) 10wt.\% ESB solution
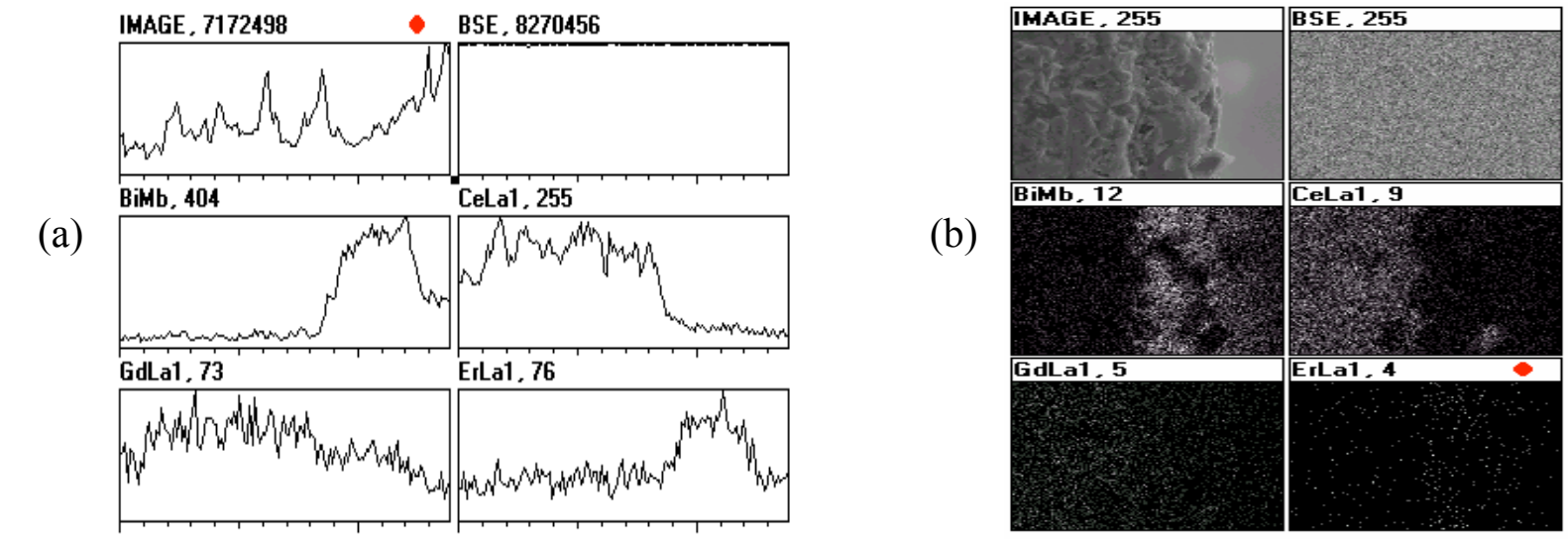

Figure 4.3.8. Chemical composition of GDC $\backslash$ ESB bilayers by X-ray (a) line-scan and (b) mapping. 


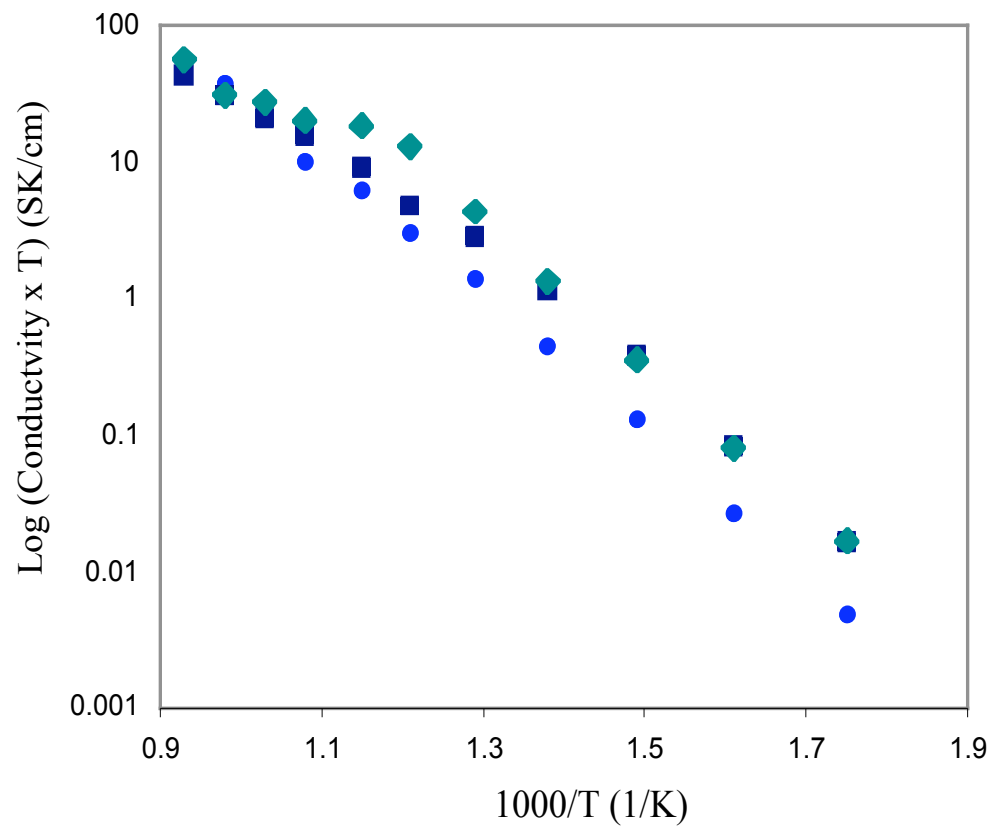

Figure 4.3.9. Total conductivity, in air, of (•) SDC (density: 90\%, uniaxial pressing, sintered at 1550 $\left.{ }^{\circ} \mathrm{C}\right),(\boldsymbol{\square}) \operatorname{SDC}\left(90 \%, \mathrm{CIP}, 1550^{\circ} \mathrm{C}\right)$ and $(\diamond) \operatorname{SDC} \backslash \mathrm{ESB}(90 \%, \mathrm{CIP})$.

than that of single layer SDC (fabricated by CIP or uniaxial pressing) for $600{ }^{\circ} \mathrm{C}<\mathrm{T}<800{ }^{\circ} \mathrm{C}$ despite the relative thinness of the ESB layer-i.e., $\sigma_{\mathrm{SDC} \text { ESB }}>\sigma_{\mathrm{SDC}(\mathrm{CIP})}>\sigma_{\mathrm{SDC}(\text { uniaxial). This result also }}$ suggests that a low conductivity (relative to SDC and ESB) phase is not formed at the SDC-ESB interface. Nevertheless, because of the thinness of the ESB layer, the increase in the effective conductivity of the bilayer over the SDC single layer is somewhat surprising and may allude to the formation of a higher conductivity ESB phase in the grain-boundary area of SDC. This is speculative, however, and requires further research for confirmation.

The change in $E_{A}$ for the conductivity of the SDC $\backslash E S B$ sample, seen at $600{ }^{\circ} \mathrm{C}$ in Fig. 4.3 .9 , may be attributed to the order-disorder transition in the oxygen sublattice of ESB at $600{ }^{\circ} \mathrm{C}$ [103], which was discussed in great detail in section 4.2.

\subsubsection{Performance of SOFCs with SDC and ESB Electrolytes}

Two SOFCs (oxygen concentration cells) of the type

$$
P_{\mathrm{O}_{2}}, \mathrm{Pt} /\left(\mathrm{CeO}_{2}\right)_{0.8}\left(\mathrm{Sm}_{2} \mathrm{O}_{3}\right)_{0.1} / \mathrm{Pt}, P_{\mathrm{H}_{2} / \mathrm{H}_{2} \mathrm{O}}
$$

and

$$
P_{\mathrm{O}_{2}}, \mathrm{Au} /\left(\mathrm{Bi}_{2} \mathrm{O}_{3}\right)_{0.8}\left(\mathrm{Er}_{2} \mathrm{O}_{3}\right)_{0.2} /\left(\mathrm{CeO}_{2}\right)_{0.8}\left(\mathrm{Sm}_{2} \mathrm{O}_{3}\right)_{0.1} / \mathrm{Pt}, P_{\mathrm{H}_{2} / \mathrm{H}_{2} \mathrm{O}}
$$


Table 4.3-1. Thicknesses of constituent layers and relative thicknesses of ESB $\backslash$ SDC electrolytes.

\begin{tabular}{ccccc}
\hline Electrolytes & SDC layer $(\mathrm{mm})$ & ESB layer $(\mu \mathrm{m})$ & Relative thickness $\left(\mathrm{L}_{\mathrm{ESB}} / \mathrm{L}_{\mathrm{SDC}}\right)$ & Cathode \\
\hline $0.8 \mathrm{ESB} \backslash \mathrm{SDC}$ & 1.601 & 0.8 & $5.0 \times 10^{-4}$ & $\mathrm{Au}$ \\
$1.3 \mathrm{ESB} \backslash \mathrm{SDC}$ & 1.756 & 1.3 & $7.4 \times 10^{-4}$ & $\mathrm{Au}$ \\
$9 \mathrm{ESB} \backslash \mathrm{SDC}$ & 1.499 & 9 & $6.0 \times 10^{-3}$ & $\mathrm{Au}$ \\
$22 \mathrm{ESB} \backslash \mathrm{SDC}$ & 1.5 & 22 & 0.015 & $\mathrm{Ag}-\mathrm{ESB}$ \\
$30 \mathrm{ESB} \backslash \mathrm{SDC}{ }^{*}$ & 1.0 & 30 & $0.03^{*}$ & $\mathrm{Au}$ \\
\hline
\end{tabular}

* relative thickness from previous results (from Wachsman et al. [112])

were used to compare the performance of the SDC \ESB bilayer vs. single-layer SDC and to test the effect of thickness ratios of the bilayer electrolyte samples. For the comparison, Au electrodes were deposited onto the ESB layer and Pt paste was coated on the SDC layer of the bilayer SDC $\backslash E S B$ electrolyte for electrodes. Each bilayer was identified as 0.8, 1.3, 9, 25, and $30 \mathrm{ESB} / \mathrm{SDC}$ by thickness of ESB layer, see Table 4.3-1, which was obtained from profilometry and SEM analysis. Oxygen partial pressure was fixed at $0.21 \mathrm{~atm}$. (using air) on the cathode side and all cells were evaluated under identical conditions of $\mathrm{H}_{2} / \mathrm{H}_{2} \mathrm{O}$ on the anode side. The open-circuit potential (OCP) of each gas concentration cell was measured over the temperature range $500{ }^{\circ} \mathrm{C}-800{ }^{\circ} \mathrm{C}$ with a gas flow rate of 60 sccm. The experimental setup is shown in Fig. 4.3.10.

In the experimental setup, a vertical inconel reactor was used to avoid severe oxidation at high temperature. The reactor consists of two mating flanges with gas tubing welded through the center of the face to allow for gas flow. The diameter of the gas tubing was $12 \mathrm{~mm}$ and the thickness of the ceramic annulus gas tubing to provide electrical insulation between the gold lead wires and the metal gas tube. The flow of gases into the reactor was regulated by calibrated mass flow controllers (Edwards 825, series B). A temperature controller was used in conjunction with a K-type thermocouple to hold the temperature in the reaction zone constant during each test. A zirconia oxygen sensor was used to measure the $P_{\mathrm{O}_{2}}$ of the reducing side thereby allowing the calculation of the theoretical (Nernst) OCP and then the ionic transference number, $t_{i}$, by comparison with the measured $\operatorname{SOFC~OCP}\left(t_{i}=\mathrm{OCP}\right.$ measured $\left./ \mathrm{OCP}_{\text {theoretical }}\right)$.

\section{Results and Discussion}

The OCP of the oxygen concentration cells described above were measured as a function of $P_{\mathrm{O}_{2}}$ on the anode side and temperature and used to calculate transference number, $t_{i}$. Figure 4.3.11 compares $t_{i}$, obtained for single layer SDC (Eq. (4.3.1)) and bilayered SDC $\backslash$ ESB (Eq. (4.3.2)) cells. The SDC $\backslash E S B$ cell showed a substantial increase in $t_{i}$, compared to the single layered, SDC-based 
SOFC cell. Also, the $t_{i}$ of the SDC $\backslash$ ESB cell was greater than that of SDC cell for every temperature. This enhancement in $t_{i}$, which is due to the ability of the ESB layer to block electronic flux, confirms the bilayer concept.

(a)

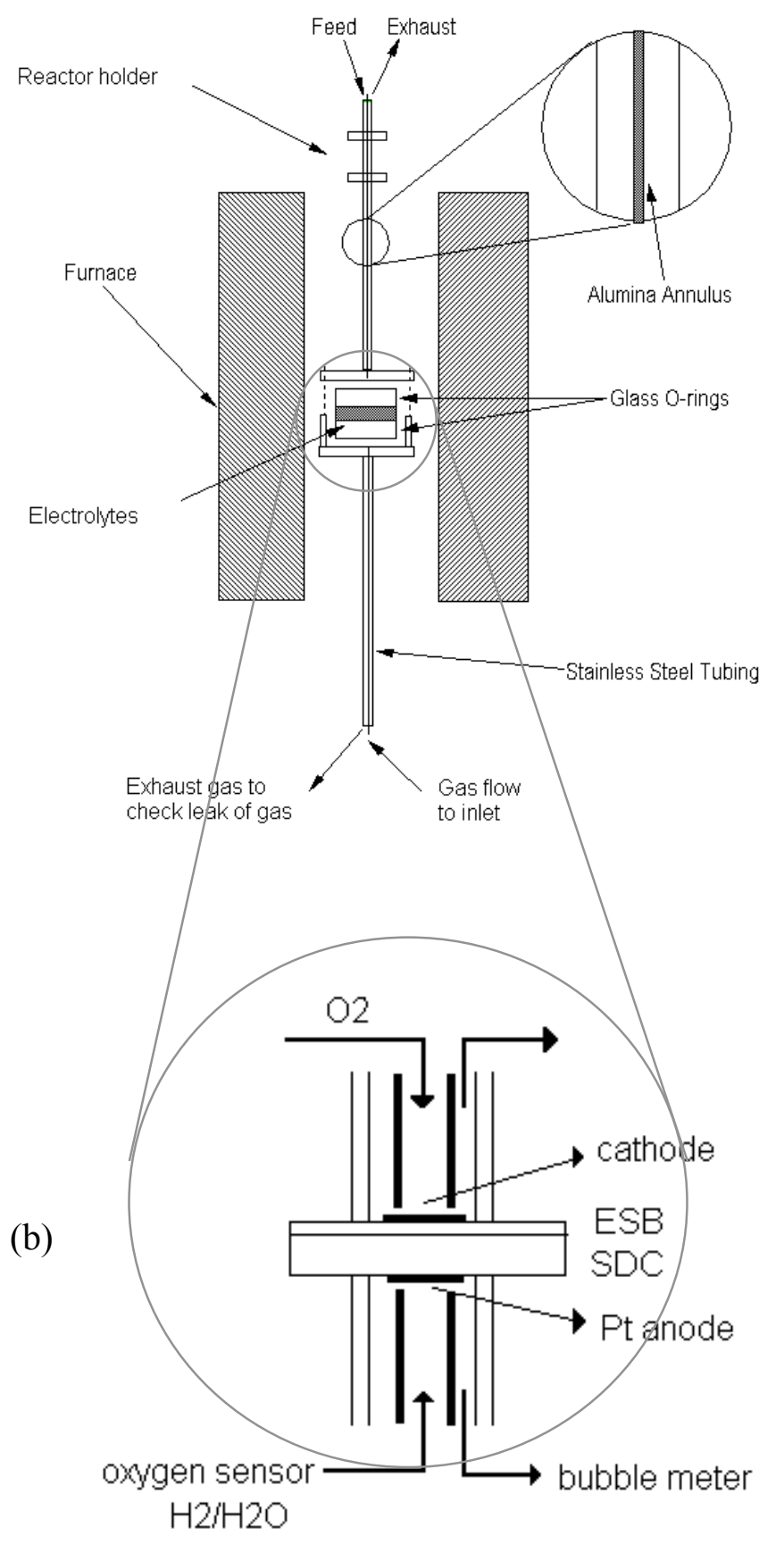

Figure 4.3.10. Experimental setup of fuel cell reactor used glass o-rings [111]. 
(a)

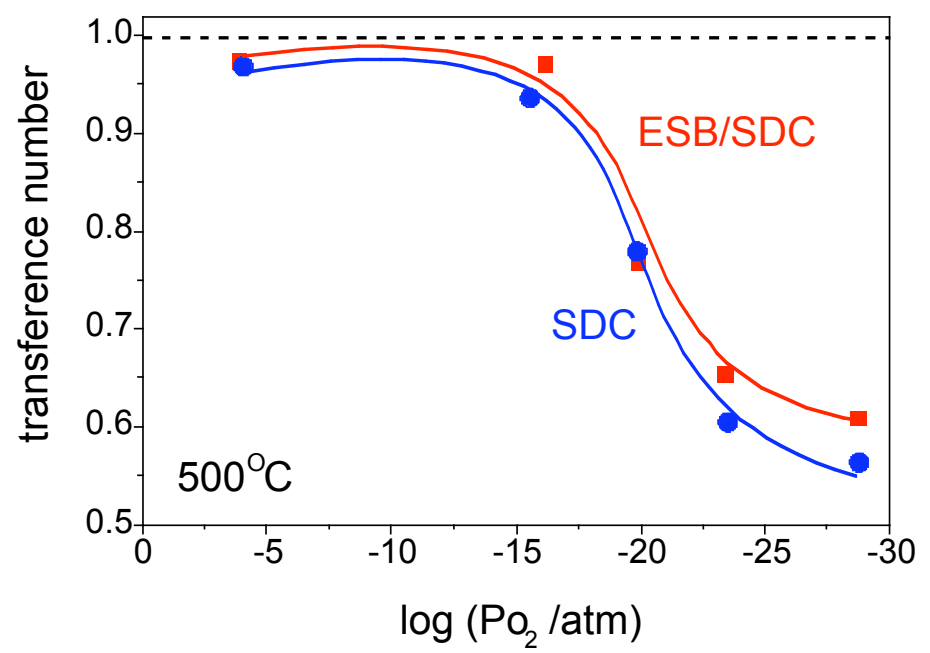

(b)

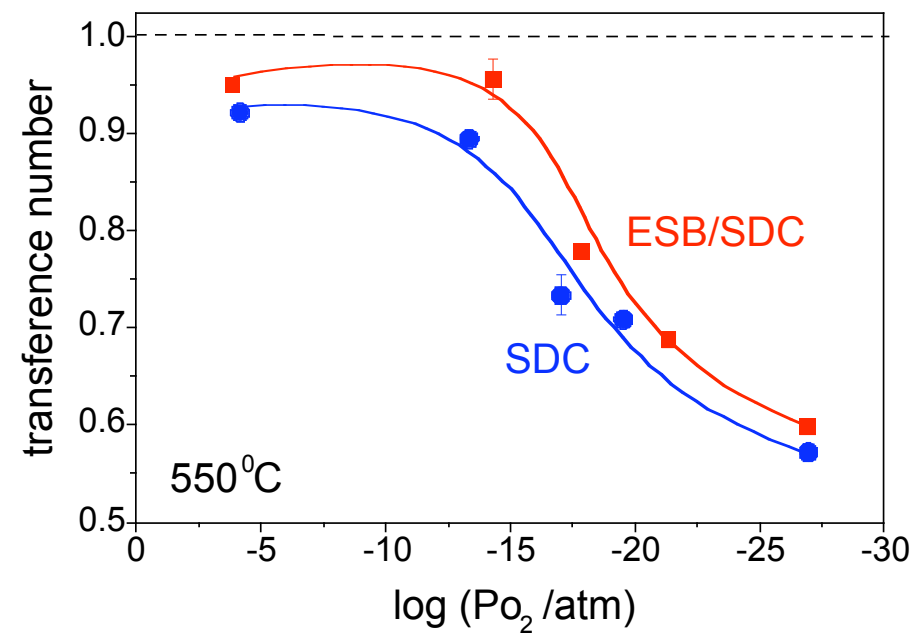

(c)

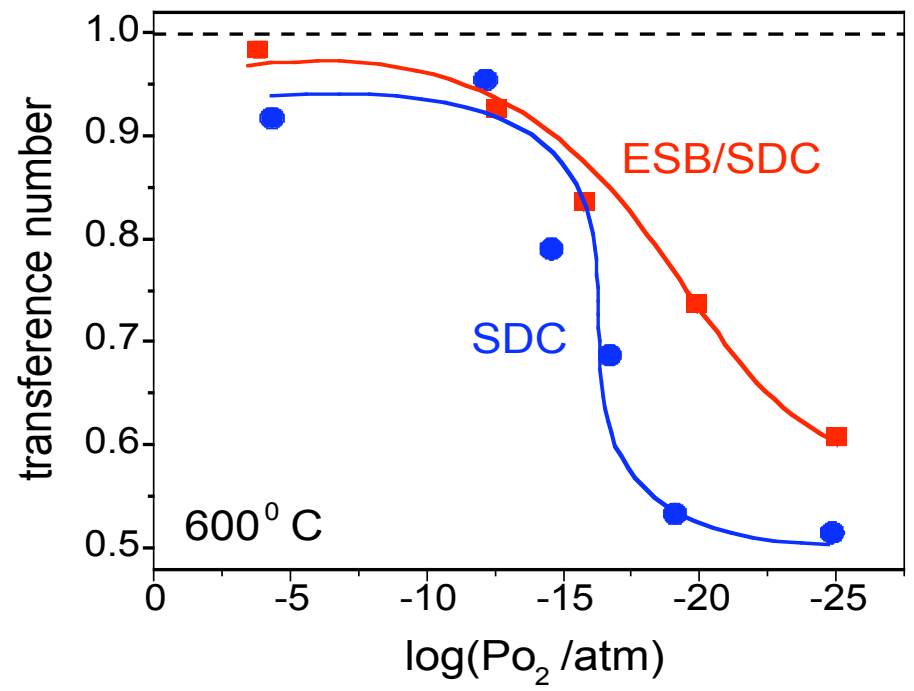


(d)

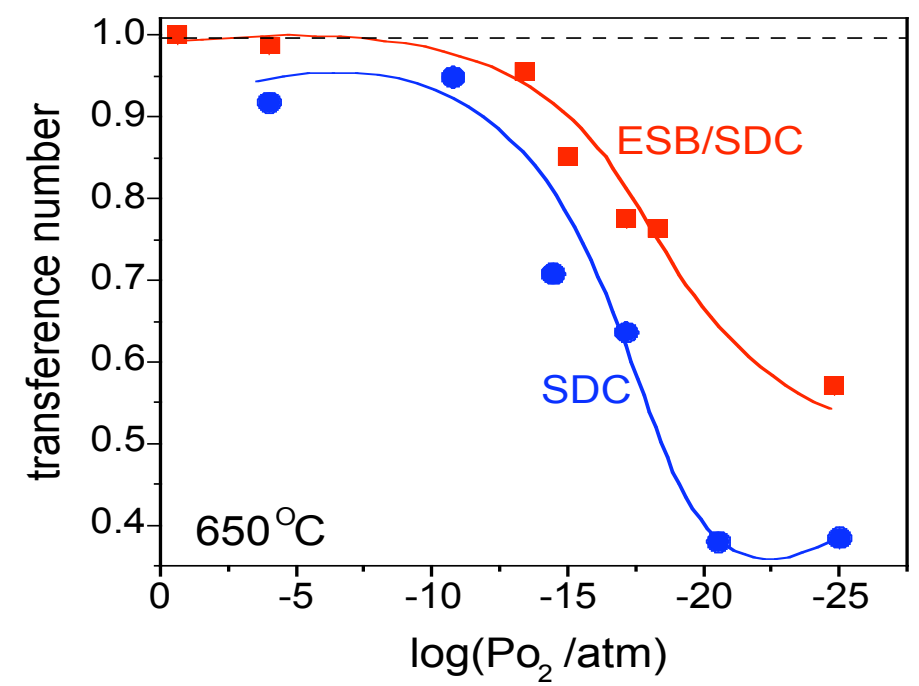

(e)
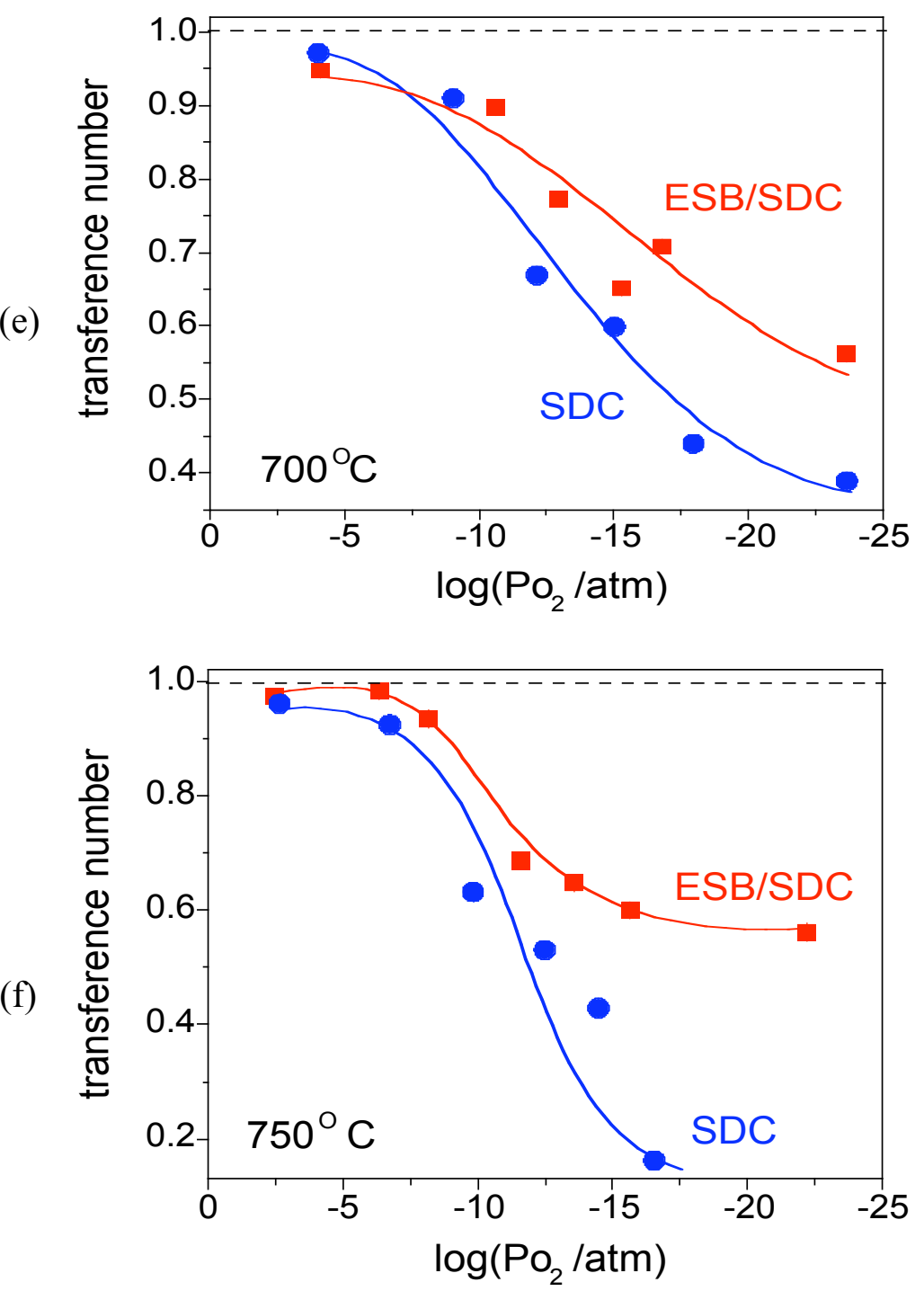


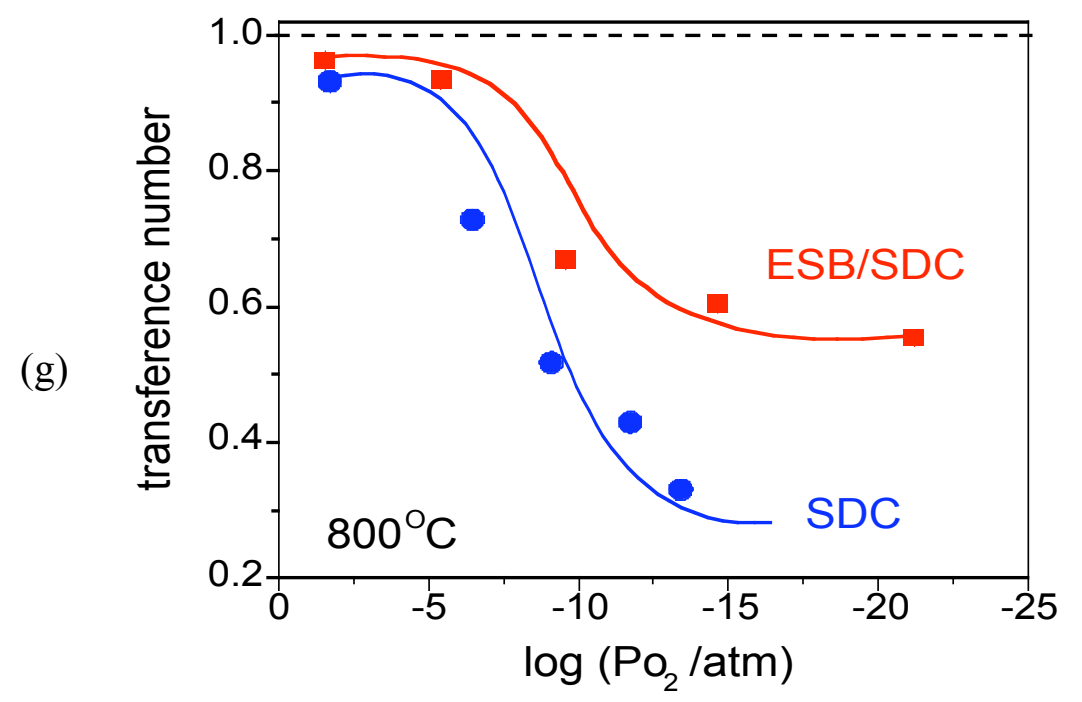

Figure 4.3.11. Comparison of $t_{i}$ of fuel cells in Eqs. (4.3.1) and (4.3.2) as a function of $P_{\mathrm{O}_{2}}$ at (a) 500 ${ }^{\circ} \mathrm{C}$, (b) $550{ }^{\circ} \mathrm{C}$, (c) $600{ }^{\circ} \mathrm{C}$, (d) $650{ }^{\circ} \mathrm{C}$, (e) $700{ }^{\circ} \mathrm{C}$, (f) $750{ }^{\circ} \mathrm{C}$, and (g) $800{ }^{\circ} \mathrm{C}$.

It is important to note that $\mathrm{CO} / \mathrm{CO}_{2}$ gas mixtures were used to control the $P_{\mathrm{O}_{2}}$ at the anode. However, $\mathrm{CO} / \mathrm{CO}_{2}$ is known for not readily attaining equilibrium in these conditions and with the electrodes used. Hence very low OCP and $t_{i}$ values were obtained from the experiment. Nevertheless, the improvement in OCP and $t_{i}$ shown by the bilayer electrolytes is not obviated since all the cells were tested under the same conditions.

Figure 4.3.12 summarizes the comparisons of the $t_{i}$ for single layer SDC and bilayer SDC $\backslash$ ESB electrolytes (the anode $P_{\mathrm{O}_{2}}$ was fixed by an $\mathrm{H}_{2} / \mathrm{H}_{2} \mathrm{O}$ ratio) as a function of temperature. The SDC $\backslash \mathrm{ESB}$ cell showed a substantial increase in OCP and $t_{i}$ compared to the uncoated SDC cell and the OCP of the SDC $\backslash$ ESB cell was greater than that of SDC at any temperature. This enhancement in OCP and $t_{i}$ is due to the ability of the ESB layer to block electronic conduction. The decrease in OCP and $t_{i}$ with increasing temperature is due to the decreasing ionic domain of the ceria electrolyte.

Results for the $t_{i}$ of SDC $\backslash E S B$ cells as a function of relative thickness are summarized in Fig. 4.3.13. From $500{ }^{\circ} \mathrm{C}$ to $800{ }^{\circ} \mathrm{C}$ the $\mathrm{OCP}$ and $t_{i}$, increase considerably with relative thickness of $\mathrm{SDC} \backslash \mathrm{ESB}$. Specifically, as the thickness ratio $\left(\mathrm{L}_{\mathrm{ESB}} / \mathrm{L}_{\mathrm{SDC}}\right)$ increases we observe that the OCP approaches the theoretical (Nernst) value and $t_{i}$ approaches unity. This trend confirms the original postulate outlined in our proposal. 

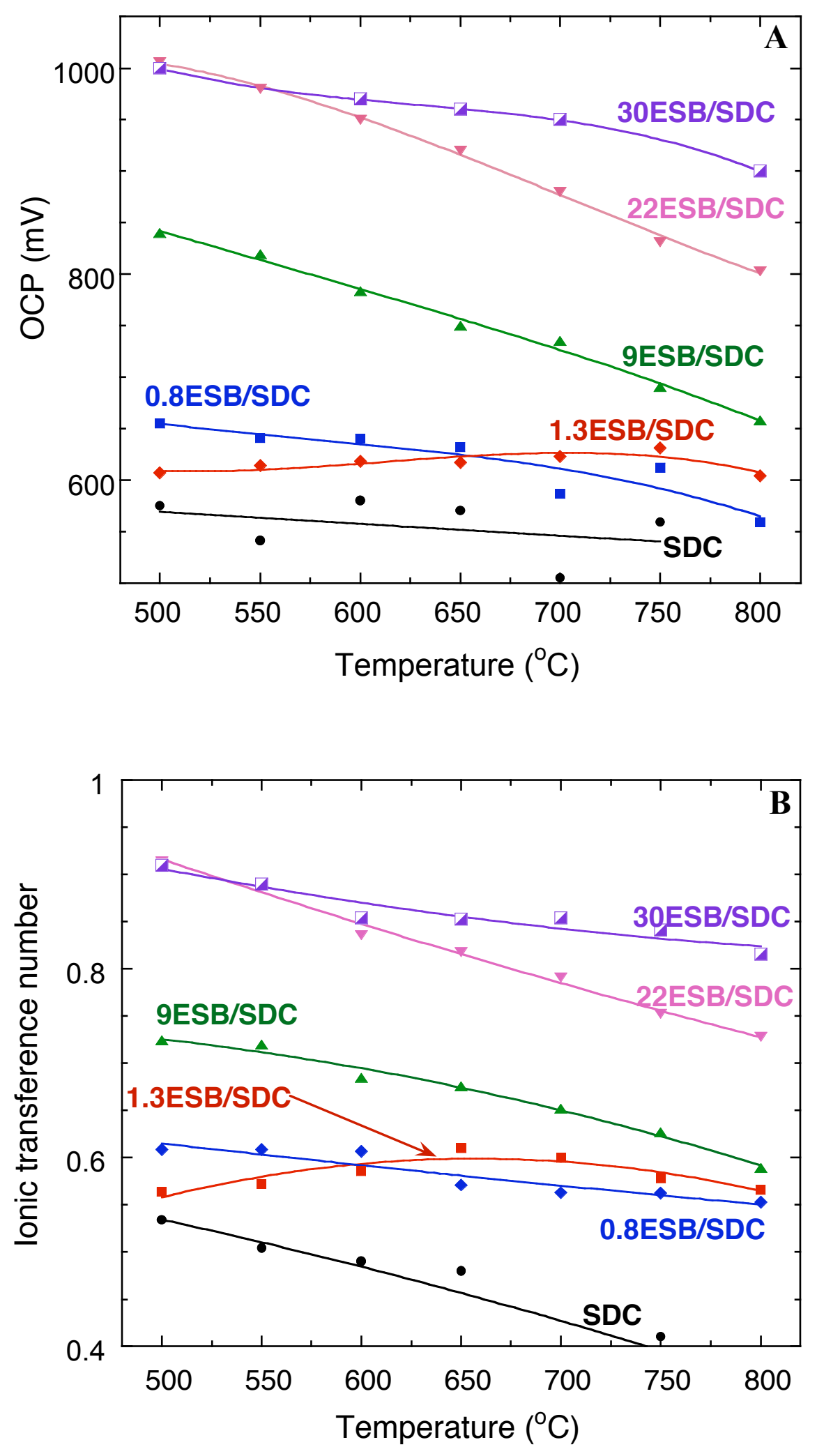

Figure 4.3.12. (a) open-circuit voltage (OCP) and (b) $t_{i}$ as a function of temperature for SOFCs with SDC and ESB/SDC electrolytes. 

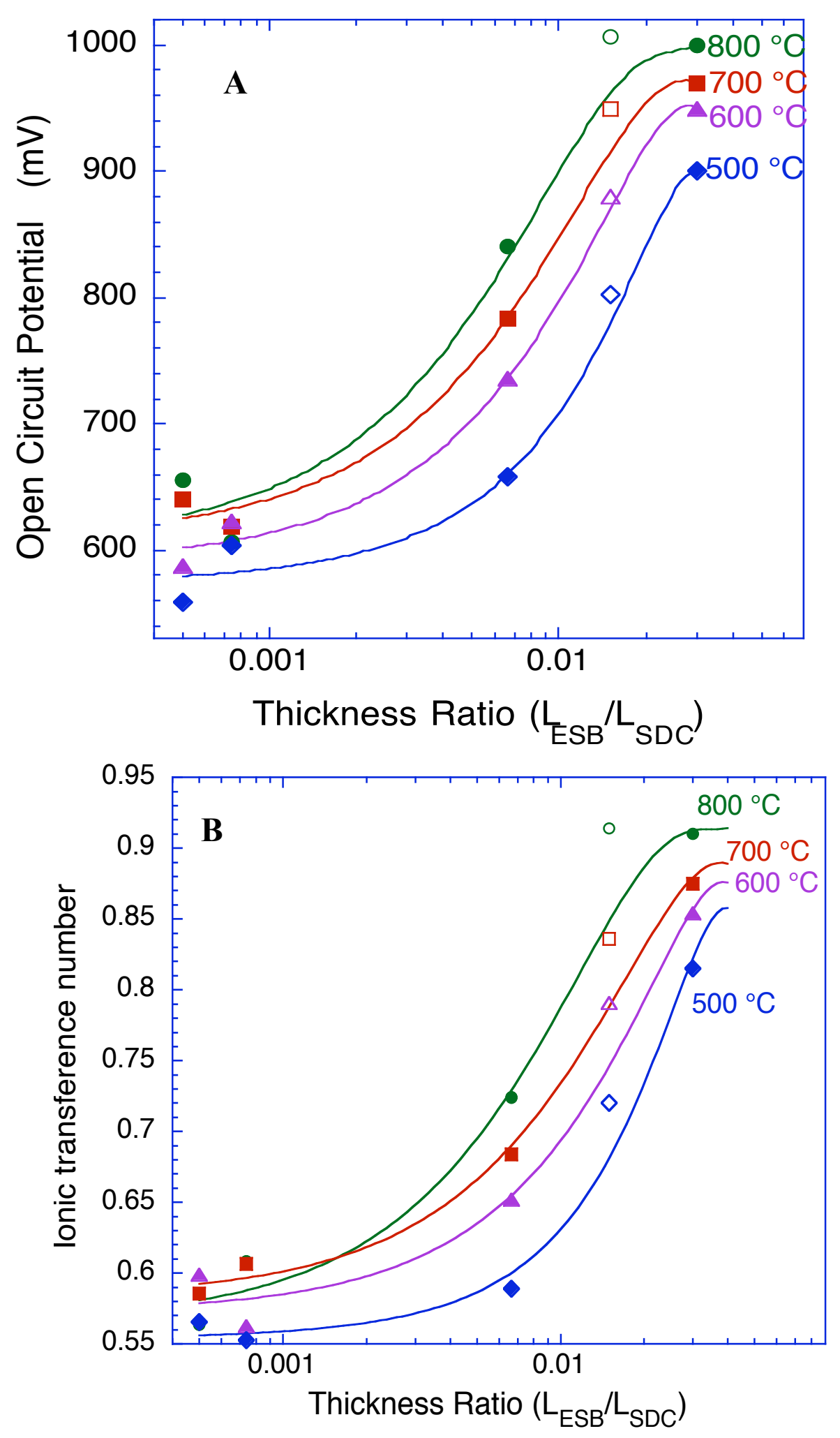

Figure 4.3.13. Effect of ESB/SDC relative thickness on (a) open-circuit voltage (OCP) and (b) ionic transference number, $t_{i}$, for bilayer electrolyte SOFC with air on the cathode side and $\mathrm{H}_{2} / \mathrm{H}_{2} \mathrm{O}$ on the anode side. Closed symbols Au cathode, open symbols Ag-ESB cathode. 


\subsection{Anode-Supported Thin Film Electrolyte SOFCs}

The results in the previous section show that increasing the bilayer thickness ratio $\left(\mathrm{L}_{\mathrm{ESB}} / \mathrm{L}_{\mathrm{SDC}}\right)$ will increase the open-circuit potential (OCP) and correspondingly the ionic transference number, $t_{i}$. However, due to differences in sintering temperature it is necessary to first fabricate an anodesupported samaria- or gadolinia doped ceria layer then add a stabilized bismuth oxide film on top of the SDC or GDC substrate.

An additional benefit of this configuration is that it allows for the fabrication of thin total thickness electrolytes, which leads to higher performance. In addition, anode supported cells show better performance than the cathode supported cells, as in general the anode polarization is lower than the cathode polarization.

As a result of time constraints, we were unable to complete our testing and optimization of the thin film GDC/ESB (bilayer) electrolytes. Hence, this section focuses primarily on the performance of an anode-supported SOFC with GDC thin film electrolytes. This was the precursor in our research plan to the development of anode-supported SOFCs with thin film GDC/ESB electrolytes. Nevertheless, at the end of this section, we briefly report preliminary results for the thin film bilayer electrolytes.

\subsubsection{Thin Film SDC Electrolytes}

Ni-Doped Ceria cermet and $\mathrm{La}_{0.6} \mathrm{Sr}_{0.4} \mathrm{Co}_{0.2} \mathrm{Fe}_{0.8} \mathrm{O}_{3}$ was chosen as the anode material and the cathode material, respectively. Acceptor-doped ceria is a mixed conductor under reducing conditions at the anode, hence the reaction zone will not be restricted to the interface of the anode and the electrolyte, leading to enhanced performance of the anode. Microstructure plays an important role in the performance of the electrodes, as shown in a recent work by Ohara et al [113]. They studied the performance of Ni-Samaria doped Ceria cermet anodes as a function of Ni content and found that at $800{ }^{\circ} \mathrm{C}$, the lowest anodic polarization $(\sim 30 \mathrm{mV})$ was achieved for a cermet with a $\mathrm{Ni}$ content of around 50 vol. \% at a current density of $300 \mathrm{~mA} / \mathrm{cm}^{2}$. Thus, in this work a NiO-Gadolinium doped Ceria (GDC) anode was fabricated with $\mathrm{Ni}$ content of 50 vol.\%. Reduction of $\mathrm{NiO}$ to $\mathrm{Ni}$ was accomplished in situ, generating $\sim 26 \%$ porosity in the structure.

Several techniques have been developed for depositing thick films on substrates from vapor deposition techniques (CVD/EVD) and pulse laser deposition (PLD) to chemical routes (sol-gel) among others [114]. However, colloidal deposition has definite advantages over these techniques in its 
simplicity, cost-effectiveness and flexibility (thickness ranging from $10-100 \mu \mathrm{m}$ can be deposited). Also, exceptional performance has been reported for SOFCs fabricated from colloidal deposition

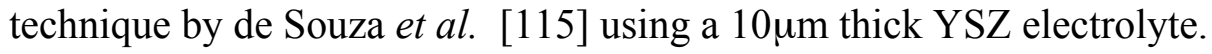

Among the cathode materials, $\mathrm{La}(\mathrm{Sr}) \mathrm{Co}(\mathrm{Fe}) \mathrm{O}_{3-\delta}(\mathrm{LSCF})$ and $\mathrm{La}(\mathrm{Sr}) \mathrm{MnO}_{3-\delta}(\mathrm{LSM})$ have been extensively studied for fuel cells based on YSZ electrolytes operating at $1000{ }^{\circ} \mathrm{C}$. LSCF is a good electronic conductor (e.g. $\mathrm{La}_{0.6} \mathrm{Sr}_{0.4} \mathrm{Co}_{0.2} \mathrm{Fe}_{0.8} \mathrm{O}_{3}-300 \mathrm{~S} / \mathrm{cm}$ at $750{ }^{\circ} \mathrm{C}$ [116]) and also shows fast oxygen surface exchange with high oxygen ion conductivity (e.g. $\mathrm{La}_{0.6} \mathrm{Sr}_{0.4} \mathrm{Co}_{0.2} \mathrm{Fe}_{0.8} \mathrm{O}_{3}-10^{-3} \mathrm{~S} / \mathrm{cm}$ at $750{ }^{\circ} \mathrm{C}$ [117]). However, LSCF reacts with YSZ to form resistive zirconate phases at temperatures as low as $800^{\circ} \mathrm{C}$. LSM is stable with YSZ up to temperatures of $1200^{\circ} \mathrm{C}$, but shows lower oxygen ionic conductivity $\left(10^{-4} \mathrm{~S} / \mathrm{cm}\right.$ at $\left.900{ }^{\circ} \mathrm{C}\right)$ that limit their application for IT-SOFCs. LSCF does not show any reactions with ceria electrolytes and hence, is a promising cathode material for IT-SOFCs based on ceria electrolytes. The Fe rich compositions are attractive as they have lower thermal expansion coefficient and hence, better matched with ceria electrolytes than Co rich compositions [118].

In this section, the fabrication of GDC thick films on NiO-GDC substrates, using a colloidal deposition has been studied. SOFCs were fabricated with $\mathrm{La}_{0.6} \mathrm{Sr}_{0.4} \mathrm{Co}_{0.2} \mathrm{Fe}_{0.8} \mathrm{O}_{3}$ as the cathode material and tested in a fuel cell configuration with air/oxygen at the cathode and moist $\mathrm{H}_{2}$ at the anode under open circuit and closed circuit conditions.

\section{Colloidal Deposition}

$11 \mathrm{~mol} \%$ Gadolinium doped Ceria powder $\left(\mathrm{GDC} \mathrm{d}_{50}=0.64 \mu \mathrm{m}\right)$ from Rhodia and $\mathrm{NiO}$ powder from Alfa-Aesar were mixed and ball milled for 24 hours with Zirconia ball media in Ethanol. The mixed NiO-GDC powder was pressed uniaxially at pressures of $39.3 \mathrm{MPa}$ to form discs of 1.25 inches diameter. The green bodies were then pre-sintered at temperatures between $800{ }^{\circ} \mathrm{C}$ to $1100{ }^{\circ} \mathrm{C}$, to coarsen the microstructure and to provide strength for further processing steps. For the colloidal suspensions, $10 \mathrm{~g}$ of GDC powder was mixed in $100 \mathrm{ml}$ of iso-propanol alcohol (IPA). Poly-vinyl Butyral (PVB) in ethanol was added to the solution as binder, equal to $5-7 \mathrm{wt} . \%$ of the oxide powder. The suspension was sonicated in an ultrasonic bath to disperse the GDC powder, with intermittent

stirring. The GDC electrolyte layer was deposited on the pre-sintered NiO-GDC discs by dip-coating and sintered at $1600{ }^{\circ} \mathrm{C}$ and $1650{ }^{\circ} \mathrm{C}$. Multiple coatings were done to get thicker films.

The pre-sintering and final sintering temperatures were varied to find the optimum combination at which the densification rate of the GDC film matches with the shrinkage rate of the NiO-GDC 
anode substrate. This helps in keeping the film under compression rather than in tension and hence, avoiding the cracking of the film due to stress built up by the 2-D confinement during sintering. The diameters of the anode discs were measured after each processing step to calculate the shrinkage profile. Anode samples were reduced in $\mathrm{H}_{2}$ atmosphere at $800{ }^{\circ} \mathrm{C}$ for 5 hours and their density was measured using the Archimedes's principle.

$\mathrm{La}_{0.6} \mathrm{Sr}_{0.4} \mathrm{Co}_{0.2} \mathrm{Fe}_{0.8} \mathrm{O}_{3}\left(\mathrm{LSCF} \mathrm{d}_{50}=0.7 \mu \mathrm{m}\right)$ from Praxiar ${ }^{\circledR}$ was mixed with $\alpha$-Terpinol, Di-butyl Pthalate, PVB and Ethanol to from the cathode paste. The paste was screen printed on the sintered GDC electrolyte film. The cathode was dried at $100{ }^{\circ} \mathrm{C}$ for $1 \mathrm{hr}$ and finally sintered at $750{ }^{\circ} \mathrm{C}$ for $1 \mathrm{hr}$.

\section{Characterization}

The samples were characterized using XRD (APD-3720) and SEM (JEOL-6400) in surface and cross-sectional view. The percentage porosity of the various GDC films was measured by stereological counting methods using point probes.

The representative microstructures of the ceria film with anode substrates pre-sintered at $800{ }^{\circ} \mathrm{C}$, $850{ }^{\circ} \mathrm{C}, 900{ }^{\circ} \mathrm{C}, 1000{ }^{\circ} \mathrm{C}, 1100{ }^{\circ} \mathrm{C}$ for $4 \mathrm{hr}$, and after coating finally sintered at $1600{ }^{\circ} \mathrm{C}$ for $6 \mathrm{hr}$ and $1650{ }^{\circ} \mathrm{C}$ for $10 \mathrm{hr}$ are shown in Figs. 4.4.1 and 4.4.2, respectively. Figure 4.4.3 shows the shrinkage profile of the NiO-GDC anode. Shrinkage did not occur for $\mathrm{T}<800{ }^{\circ} \mathrm{C}$. Figure 4.4 .4 shows the percentage porosity of the GDC film under different sintering profiles. The volume fraction of the porosity in the microstructure is calculated by taking the average fraction of points on a grid, lying on the pores.

The electrodes need to be porous enough to allow for the gaseous diffusion and the electrolyte needs to be highly dense to act as a barrier for the fuel gases and air. The density of the anode substrate after sintering at $1600{ }^{\circ} \mathrm{C}$ was measured to be $6.72 \mathrm{gm} / \mathrm{cc}(\sim 97 \%$ of the theoretical density). On exposure to $\mathrm{H}_{2}$ atmosphere at $800{ }^{\circ} \mathrm{C}, \mathrm{NiO}$ in the anode reduced to $\mathrm{Ni}$, which lead to an open porosity of $\sim 20 \%$ and a total porosity of $\sim 29 \%$ in the structure. The anode with an open porosity of $\sim 20 \%$ may be sufficiently porous, but it would be better to increase the porosity of the anode by adding pore-formers. Figure 4.4.5 (a), (b) show the microstructure of the LSCF cathode sintered at $750{ }^{\circ} \mathrm{C}$ for 1 hour in the surface and cross-sectional view. Even at this low sintering temperature the cathode looks to have modest porosity, so further work needs to be done to optimize the microstructure. 


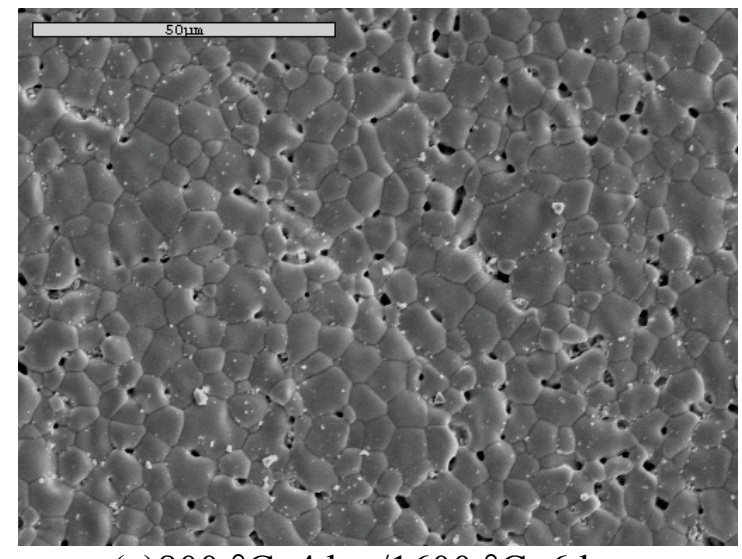

(a) $800{ }^{\circ} \mathrm{C}, 4 \mathrm{hrs} / 1600^{\circ} \mathrm{C}, 6 \mathrm{hrs}$

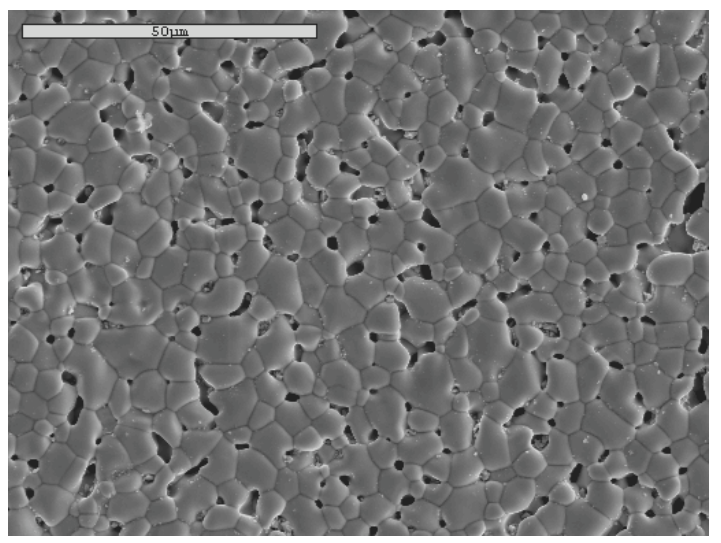

(c) $900{ }^{\circ} \mathrm{C}, 4 \mathrm{hrs} / 1600{ }^{\circ} \mathrm{C}, 6 \mathrm{hrs}$

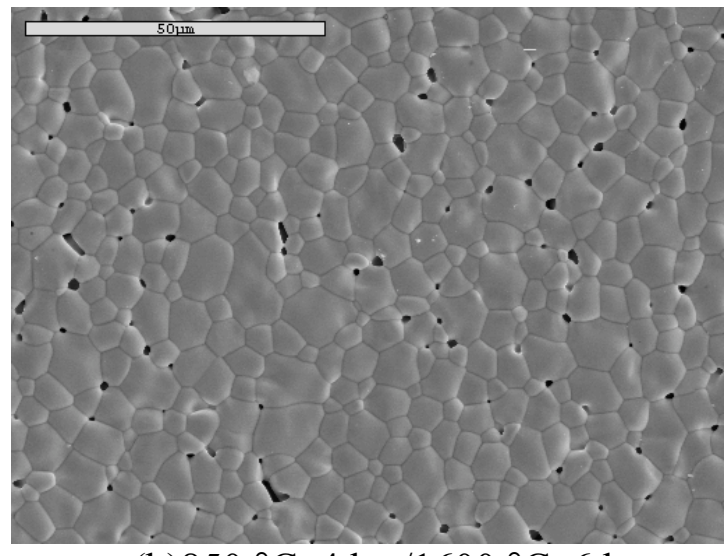

(b) $850{ }^{\circ} \mathrm{C}, 4 \mathrm{hrs} / 1600{ }^{\circ} \mathrm{C}, 6 \mathrm{hrs}$

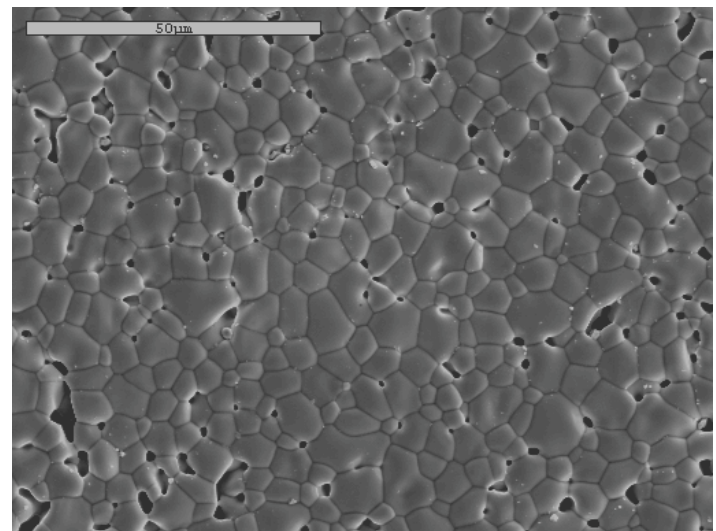

(d) $1000{ }^{\circ} \mathrm{C}, 4 \mathrm{hrs} / 1600{ }^{\circ} \mathrm{C}, 6 \mathrm{hrs}$

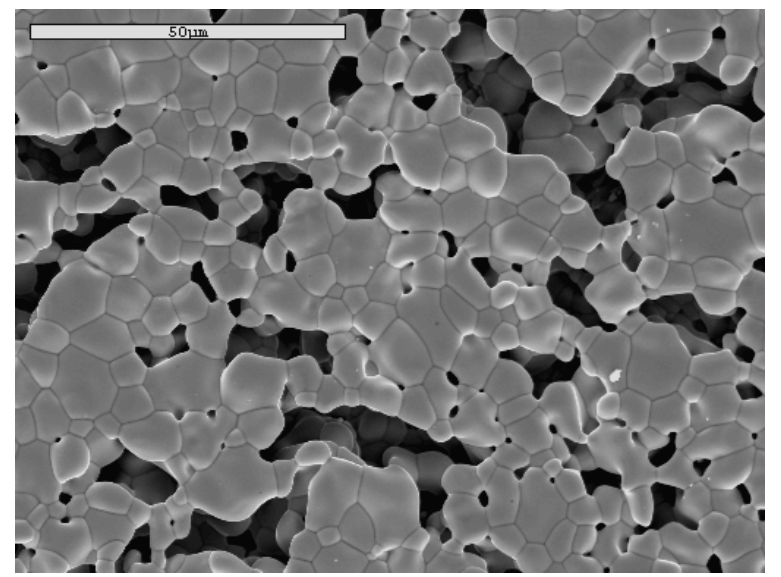

(e) $1100{ }^{\circ} \mathrm{C}, 4 \mathrm{hrs} / 1600{ }^{\circ} \mathrm{C}, 6 \mathrm{hrs}$

Figure 4.4.1. (a)-(e) Representative microstructures of the GDC film under different pre-sintering conditions. Final sintering was at $1600^{\circ} \mathrm{C}$. 


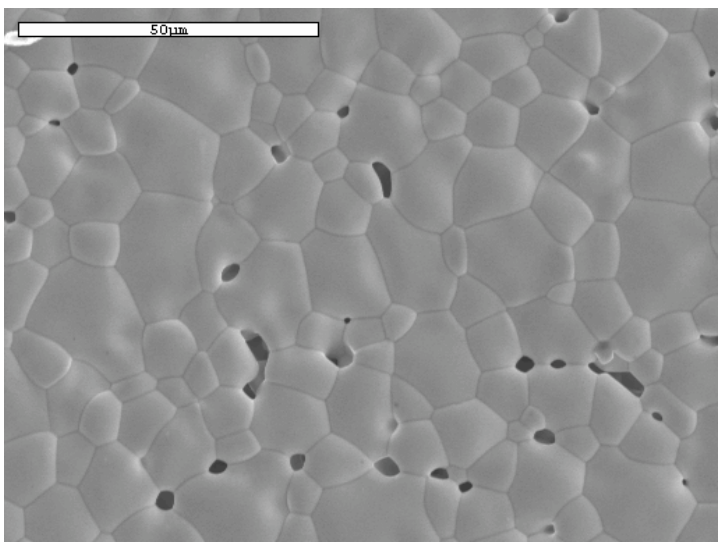

(a) $800{ }^{\circ} \mathrm{C}, 4 \mathrm{hrs} / 1650{ }^{\circ} \mathrm{C}, 10 \mathrm{hrs}$

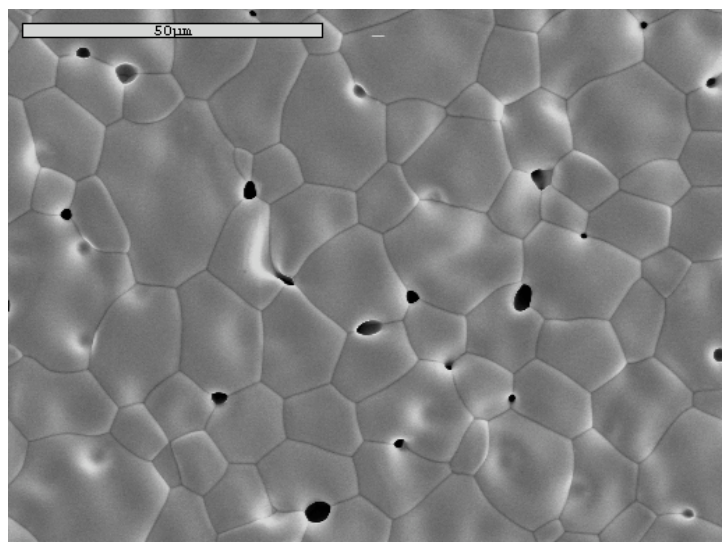

(c) $900{ }^{\circ} \mathrm{C}, 4 \mathrm{hrs} / 1650{ }^{\circ} \mathrm{C}, 10 \mathrm{hrs}$

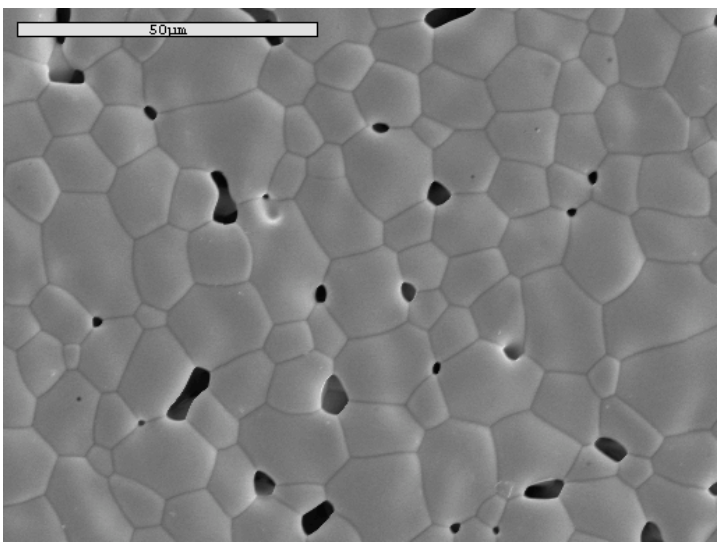

(b) $850{ }^{\circ} \mathrm{C}, 4 \mathrm{hrs} / 1650{ }^{\circ} \mathrm{C}, 10 \mathrm{hrs}$

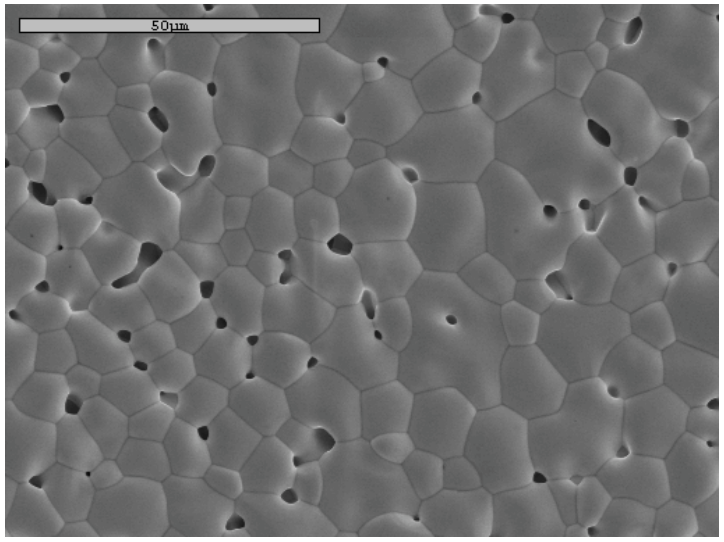

(d) $1000{ }^{\circ} \mathrm{C}, 4 \mathrm{hrs} / 1650{ }^{\circ} \mathrm{C}, 10 \mathrm{hrs}$

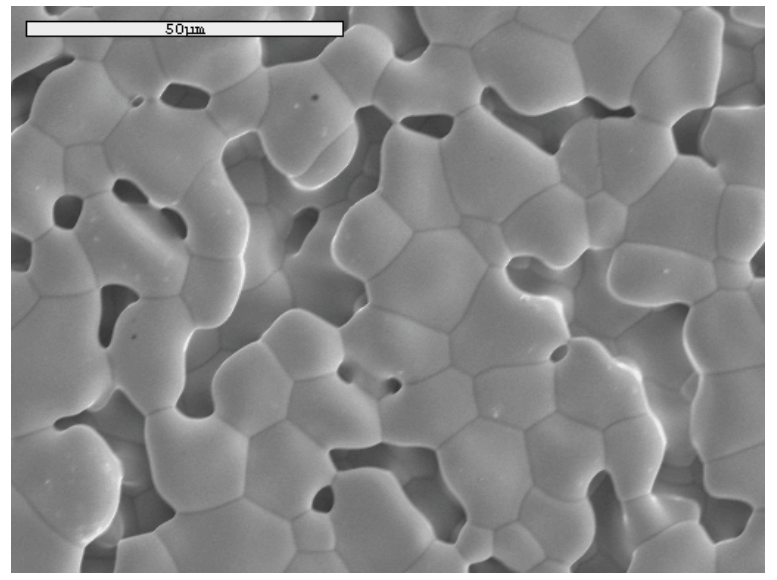

(e) $1100{ }^{\circ} \mathrm{C}, 4 \mathrm{hrs} / 1650{ }^{\circ} \mathrm{C}, 10 \mathrm{hrs}$

Figure 4.4.2. (a)-(e) Representative microstructures of the GDC film under different pre-sintering conditions. Final sintering was at $1650^{\circ} \mathrm{C}$. 


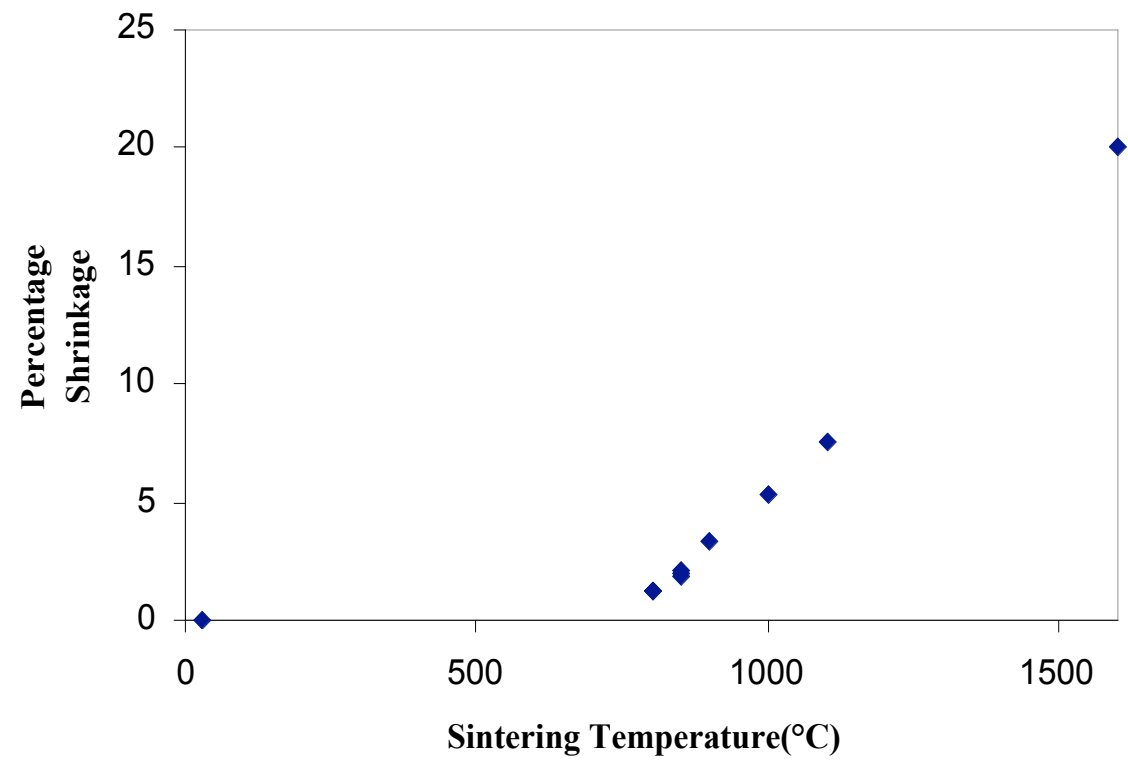

Figure 4.4.3. Shrinkage profile of the NiO-GDC anode with sintering temperature.

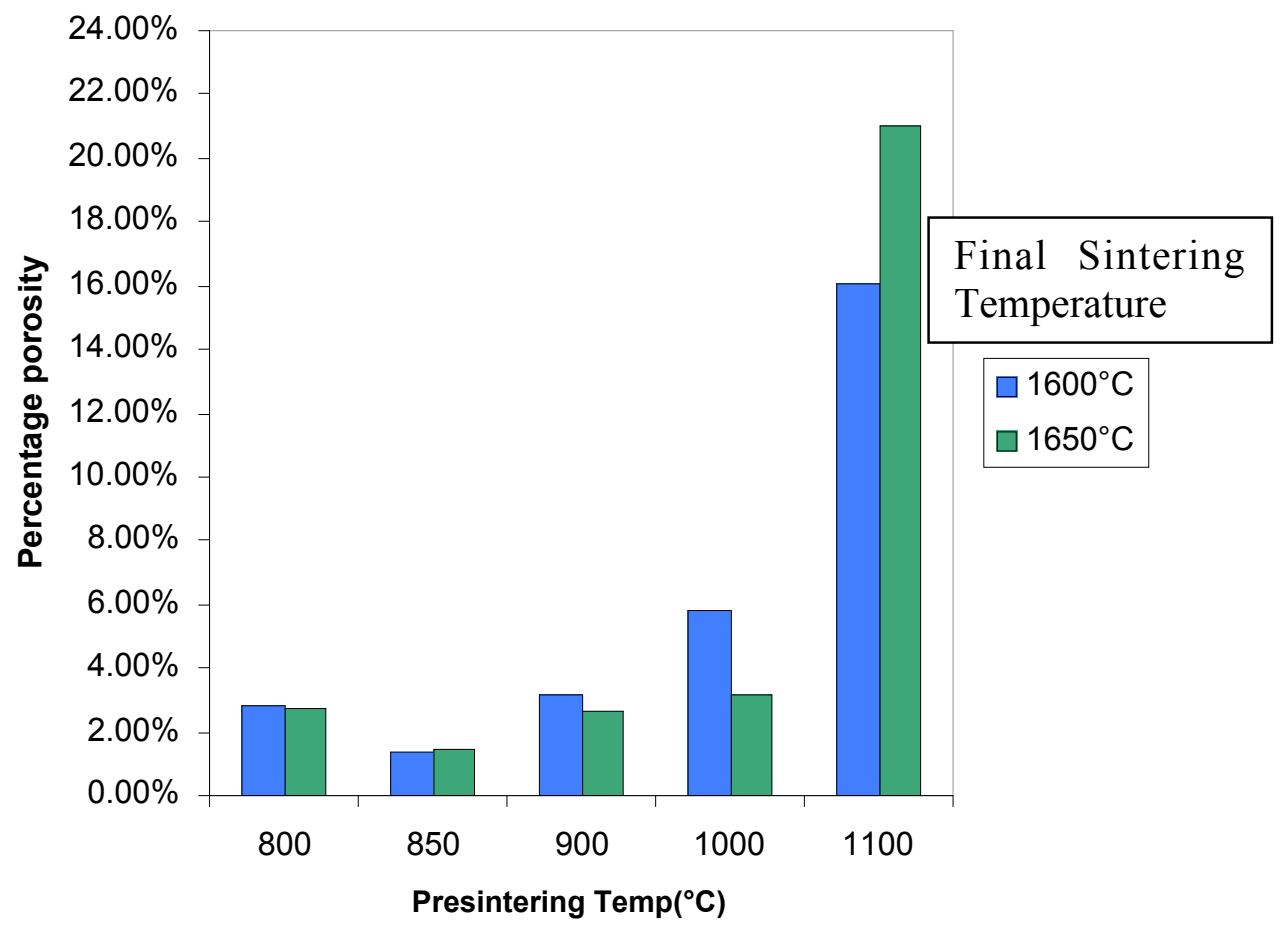

Figure 4.4.4. Porosity in the GDC film as a function of the pre-sintering and final sintering temperature of the anode. 
(a)

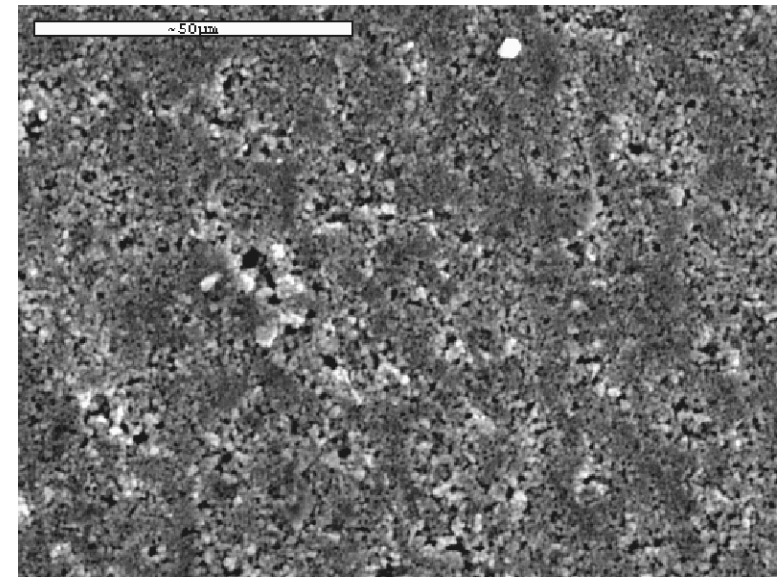

(b)

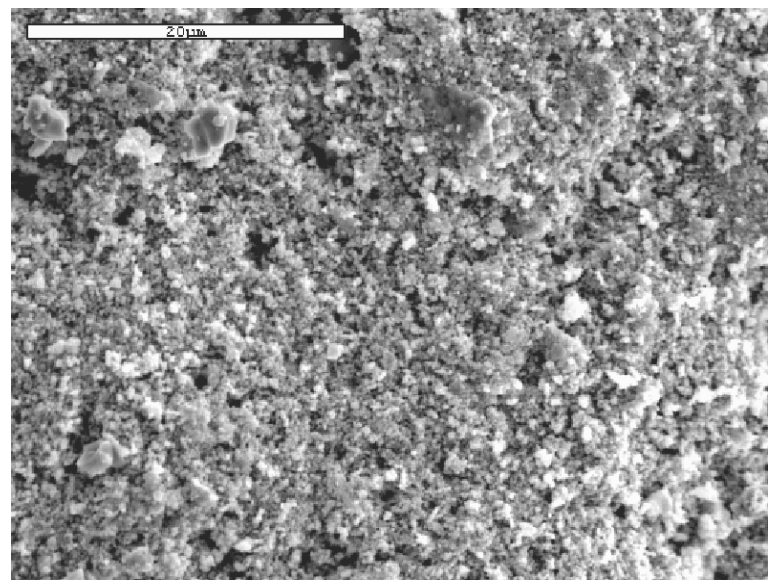

Figure 4.4.5. SEM pictures of the LSCF cathode: (a) Surface (2500X), (b) Cross-section (2000X).

As Fig. 4.4.4 shows, varying the pre-sintering temperature lead to a minima in porosity in the GDC film at $850{ }^{\circ} \mathrm{C}$ for both the final sintering temperatures of $1600{ }^{\circ} \mathrm{C}$ and $1650{ }^{\circ} \mathrm{C}$, with values of $1.38 \%$ and $1.48 \%$ respectively. However, as can be seen in the Fig. 4.4.2, sintering at $1650{ }^{\circ} \mathrm{C}$ leads to coarsening of the microstructure. Both the grain and pore size increase and become almost the double the size to that sintered at $1600^{\circ} \mathrm{C}$. Bigger pore size in the electrolyte microstructure has larger chances of failure in partitioning the fuel and air atmospheres in comparison. Also, thermodynamic studies on film stability have shown that, the film thickness should be larger than the grain size of the film for the film to be stable [116]. Else, the film would lower its energy by breaking into isolated islands and exposing the substrate. Hence, it was decided to work with films pre-sintered at $850{ }^{\circ} \mathrm{C}$ and finally sintered at $1600{ }^{\circ} \mathrm{C}$ for OCP and I-V measurements in a fuel cell configuration.

Density of the GDC film is directly influenced by the stability of the sol, Fig. 4.4.6. Increasing the stability of the sol by adding more PVB (7 wt\%), leads to an increase in the density of the film at the same sintering conditions as seen in Fig. 4.4.6 (a). Figures 4.4.6 (b) and (c) shows the crosssectional SEM of the GDC film with 5 coats $(\sim 10 \mu \mathrm{m})$ and $15(\sim 30 \mu \mathrm{m})$ coats, respectively. The micro-structure has a few closed porosities but no pin holes. Figure 4.4.7 shows the fractured crosssection of a tested unit cell with the reduced Ni-GDC anode, GDC electrolyte $(\sim 15 \mu \mathrm{m})$ and LSCF cathode $(\sim 85 \mu \mathrm{m})$.

\section{Performance}

LSCF/GDC/NiO-GDC samples were tested in a fuel cell configuration with air or oxygen at the cathode side and $\mathrm{H}_{2} / 3 \% \mathrm{H}_{2} \mathrm{O}$ at the anode side with flow rates of $10-30 \mathrm{sccm}$ at temperatures between 


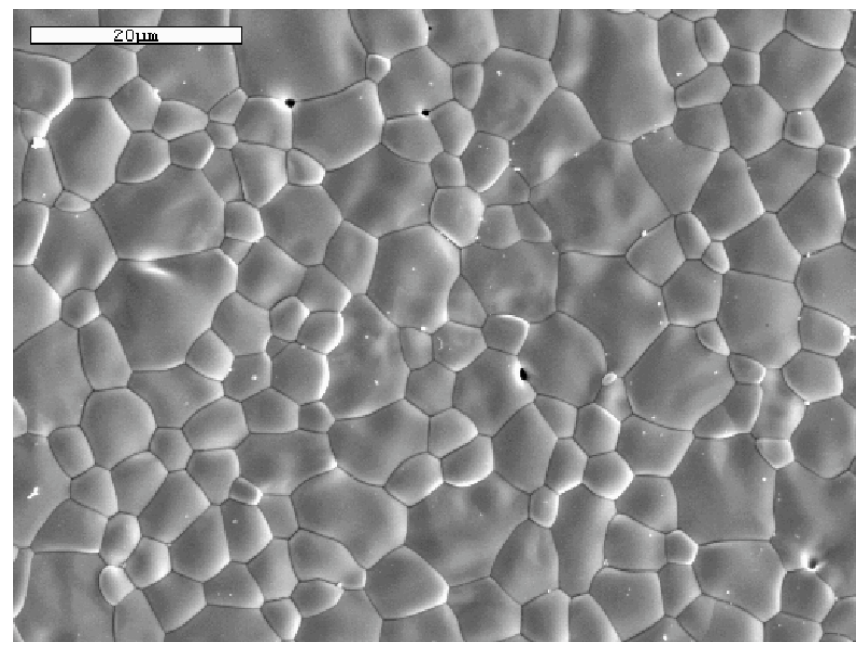

(a) $850{ }^{\circ} \mathrm{C}, 4 \mathrm{hrs} / 1600{ }^{\circ} \mathrm{C}, 6 \mathrm{hrs}$

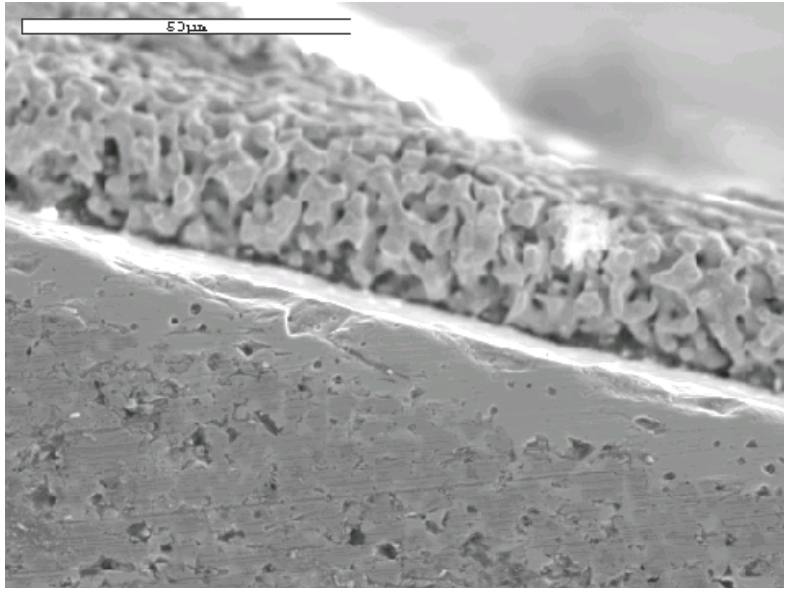

(b) $850{ }^{\circ} \mathrm{C}, 4 \mathrm{hrs} / 1600{ }^{\circ} \mathrm{C}, 6 \mathrm{hrs}$ with 5 coats*

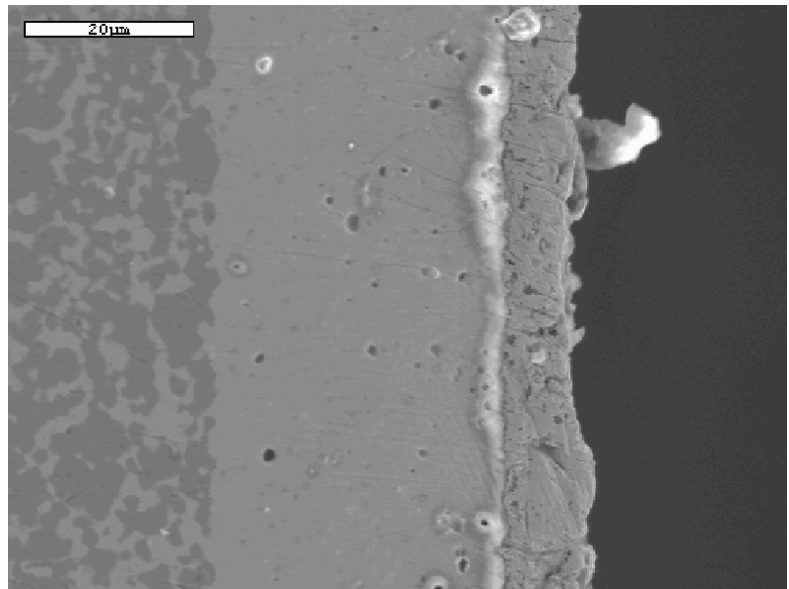

(c) $850{ }^{\circ} \mathrm{C}, 4 \mathrm{hrs} / 1600^{\circ} \mathrm{C}, 6$ hrs with 15 coats

Figure 4.4.6. Increased PVB concentration in the GDC sol (a) Surface microstructure of the film (b),(c) Crosssectional microstructures of the film with 5 and 15 coats, respectively. (* with reduced anode)

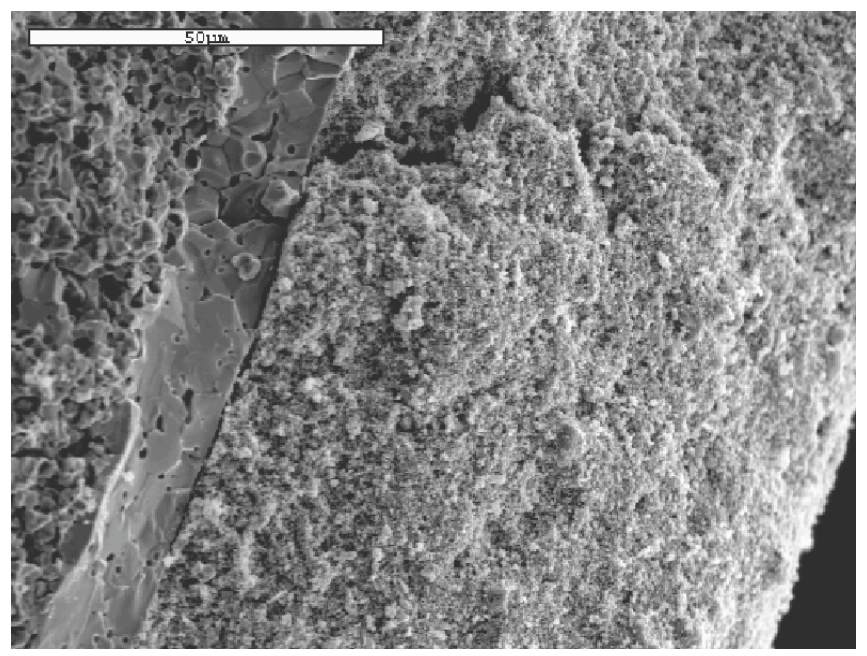

Figure 4.4.7. Fractured cross-section of a tested cell: Ni-GDC/ GDC $(\sim 15 \mu \mathrm{m}) / \mathrm{LSCF}(\sim 85 \mu \mathrm{m})$. 


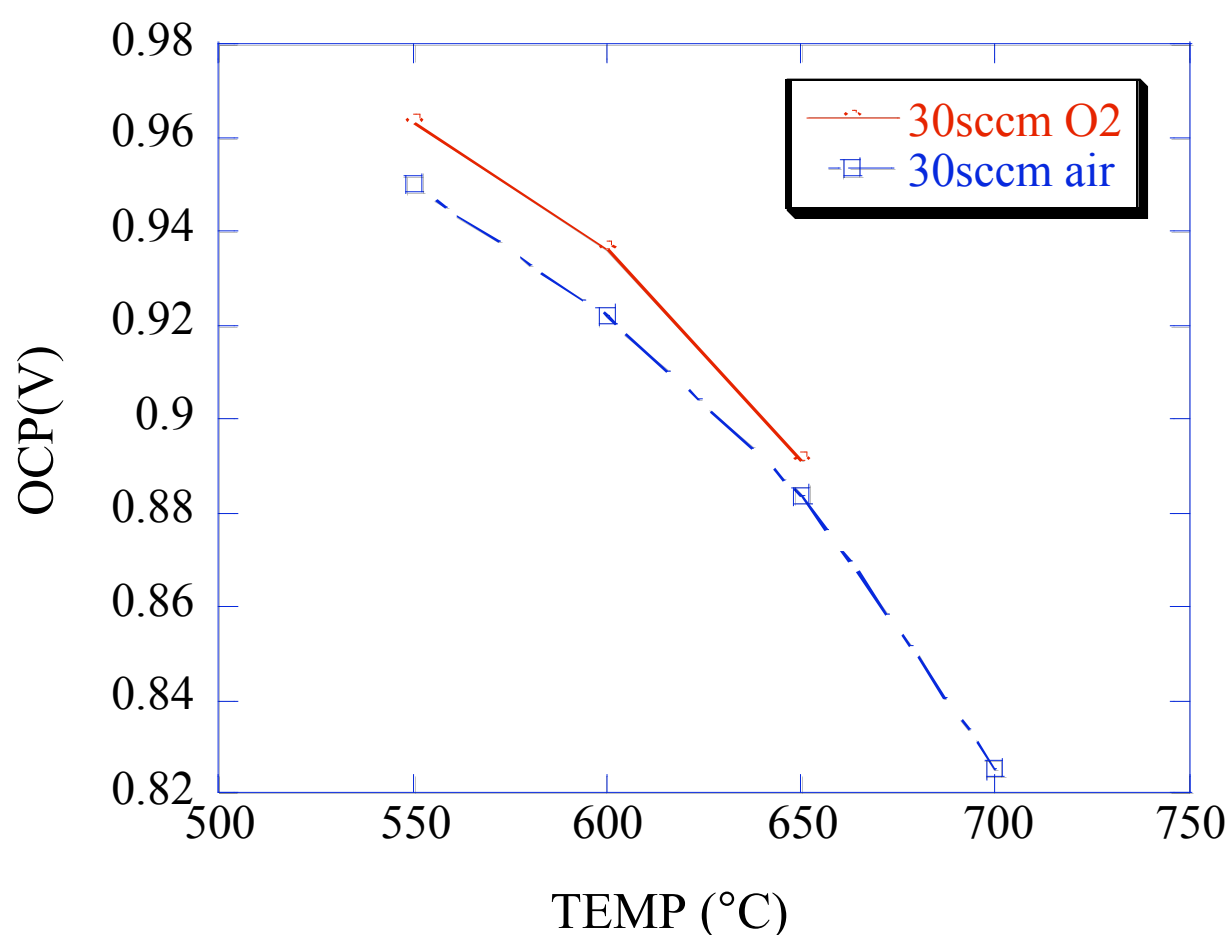

Figure 4.4.8. OCP of the cell as a function of temperature, cathode gas atmosphere and the flow rate.

$500{ }^{\circ} \mathrm{C}$ and $700{ }^{\circ} \mathrm{C}$. The flow rate at the anode and cathode side was kept equal. The open circuit potential (OCP) of the cell was measured using a Keithley multi-meter and the I-V characteristics of the cell were measured using a Solatron Potentiostat/Galvanostat (SI-1287). After an initial transient on exposing the sample to the reducing $\mathrm{H}_{2}$ atmosphere, due to the reduction of $\mathrm{NiO}$ to $\mathrm{Ni}$, the $\mathrm{OCP}$ increased and attained a stable value within a few minutes. For the I-V characteristics each data point was collected after 15 minutes to attain a stable value.

The I-V and power density of the cell were measured as a function of temperature, the cathode gas atmosphere and flow rate. Figure 4.4.8 is a plot of OCP vs. temperature. At higher temperatures, the electrolytic domain of ceria reduces and the ionic transference number of ceria decreases. Hence, the OCP of the cell decreases at higher temperatures. As expected, the cell showed a higher OCP with oxygen at the cathode, in comparison to air. Increasing the anode flow rate lowers the effective $P_{\mathrm{O}_{2}}$ at the anode thereby yielding a higher OCP.

Figure 4.4.9 shows the I-V characteristics and power density of the cell with air at the cathode. In open circuit conditions, the cell potential decreased with increasing temperature (same as shown in Fig. 4.4.8). The cell potential also decreased on drawing a current from the cell. The shape of the I-V curves became more linear with increasing temperature. This may be due to lower activation polarization losses at the electrodes at higher temperature. In cells with ceria electrolytes, the power 

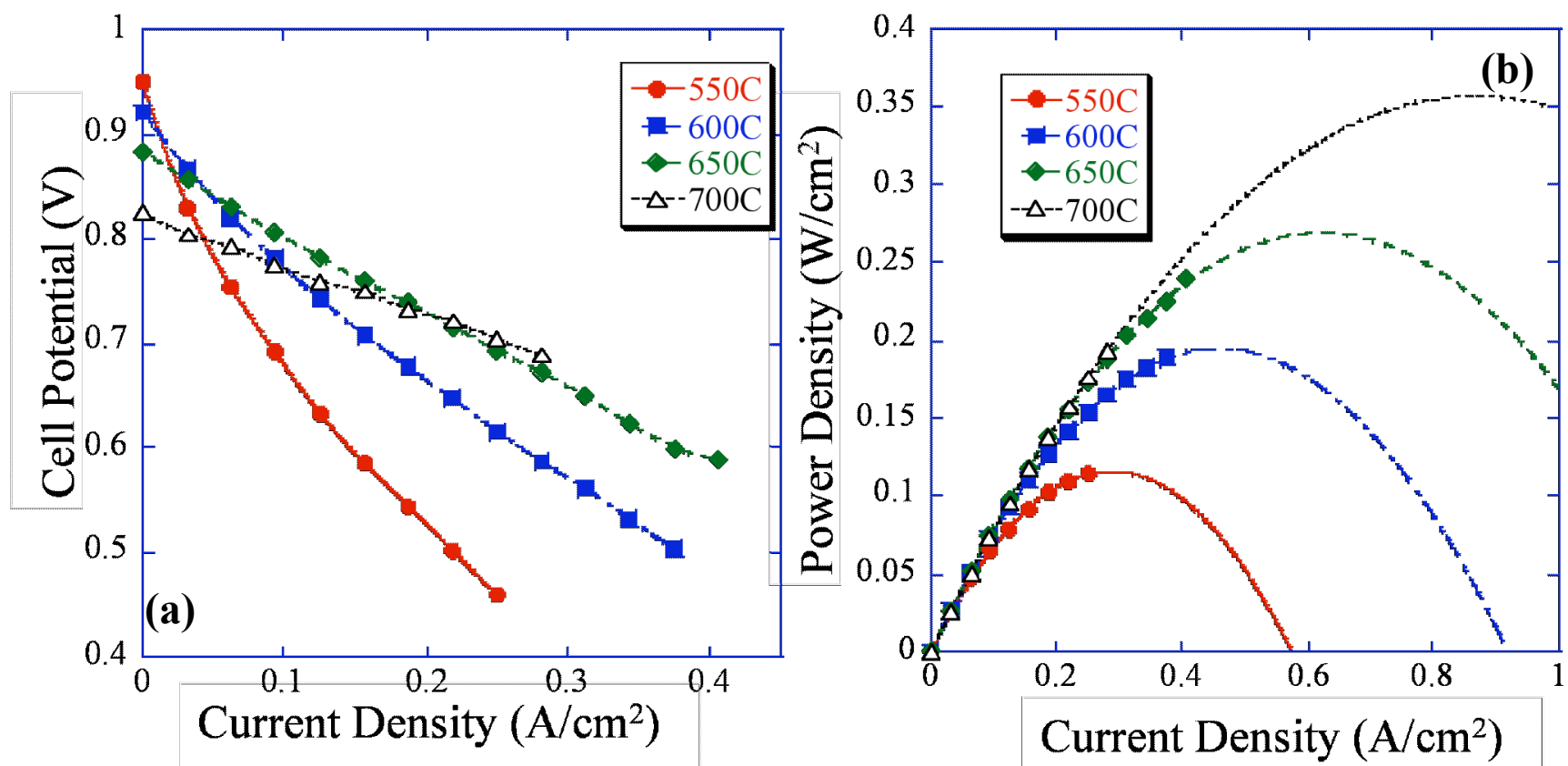

Figure 4.4.9. (a) $\mathrm{I}-\mathrm{V}$ characteristics and (b) power density of fuel cell with thin film ceria electrolyte as a function of current density and temperature.

density depends on the conflicting parameters of cell potential and ASR. On increasing the temperature, the cell potential and the ASR both decrease. Hence, there is an optimal temperature where maximum power density is achieved. At $700{ }^{\circ} \mathrm{C}$, Fig. $4.4 .9 \mathrm{~b}$, with a flow rate of $30 \mathrm{sccm}$, the cell showed an extrapolated power density of $0.37 \mathrm{~W} / \mathrm{cm}^{2}$ at a current density of $0.9 \mathrm{~A} / \mathrm{cm}^{2}$. In general, the obtained power densities were lower than they could be because of the reduced OCP, which is a result of electronic conduction. An ESB layer would raise the OCP, resulting in greater power densities at all temperatures.

The performance of the cell was compared with pure oxygen at the cathode (not shown) instead of air. Similar trends were observed in the I-V characteristics and power density of the cell with oxygen at the cathode. The cell with oxygen showed a higher power density $\left(0.42 \mathrm{~W} / \mathrm{cm}^{2}\right.$ at 0.9 $\mathrm{A} / \mathrm{cm}^{2}, 700{ }^{\circ} \mathrm{C}$ ) compared to the cell with air at cathode. At the same temperature and flow rate, the cell showed a lower ASR with oxygen than with air. Hence, it seems that the performance of the cell was limited by the performance of the cathode. This phenomenon may be due to high density of the cathode or due to decreased p-type conductivity of LSCF at low oxygen partial pressures.

Overall, the cell performance of the SOFC with thin film ceria electrolyte was comparable to those reported in the literature [119]. The cathode performance needs improvement and we are 
developing new cathodes to address this. Further, depositing an electronic blocking layer of ESB on the GDC electrolyte will improve the performance of the cell at intermediate temperatures.

\subsubsection{Thin Film GDC|ESB Bilayer Electrolytes}

The colloidal route was also taken for the fabrication of the ESB films on top of the GDC layer. However, in addition to the difference in sintering temperature, there is a difference between the sintering process of the ESB and the GDC layers. In the case of GDC, the NiO-GDC substrate shrinks as the GDC film sinters while in the case of ESB, the non-shrinking GDC layer constrains the sintering of the ESB layer. However, the low melting point $\left(890^{\circ} \mathrm{C}\right)$ of ESB helps in fabricating a dense ESB film.

$\mathrm{Bi}_{1.6} \mathrm{Er}_{0.4} \mathrm{O}_{3}(\mathrm{ESB} 20)$ powder was made using an amorphous citrate process. Nitrates in stoichiometric ratios were dissolved in DI water with nitric acid. Citric acid was added equal to 1.5 times the moles of metal cations in the solution. The solution was heated at $90-100{ }^{\circ} \mathrm{C}$ on a hot plate to gel and later formed a solid foam, which was then calcined at temperatures between $500-700{ }^{\circ} \mathrm{C}$. For the colloidal suspensions, $7 \mathrm{~g}$ of ESB20 powder (calcined at $500^{\circ} \mathrm{C}$ ) was mixed in $25 \mathrm{ml}$ of IPA. PVB in ethanol was added to the solution as binder, equal to $5 \mathrm{wt} \%$ of the oxide powder. The suspension was sonicated in an ultrasonic bath to disperse the ESB20 powder, with intermittent stirring. The ESB20 electrolyte layer was deposited on top of the co-sintered GDC11(film)/NiO-GDC11(substrate) by dip-coating and finally sintered at $890^{\circ} \mathrm{C}$. Multiple coatings were done to get thicker films.

Initial trials of depositing a dense ESB layer were done as described in section 4.4.1 for ceria coatings. Figure 4.4.10 shows the cross-section of an anode supported bilayer electrolyte. The ESB film appears dense and does not seem to have interconnected porosity. The amorphous citrate route used for processing leads to agglomerated powder, which reduces the stability of the sol and also reduces the sinterability of the film. Fine particle size is critical for thick film processing by colloidal route and hence, other powder processing routes like co-precipitation may improve the density further.

In order to test the bilayer electrolyte compatible cathodes are needed. We have not yet developed such cathodes, hence the potential performance of a SOFC with a bilayer electrolyte was extrapolated from our performance data for the SOFC with thin film ceria electrolyte, as given in section 4.4.1. The extrapolation was based solely on the possibility of attaining near theoretical opencircuit potential for a bilayer electrolyte. The reduction in electrolyte resistance as a result of introducing an ESB layer was ignored. (using the bilayer ASR obtained from our modeling work 


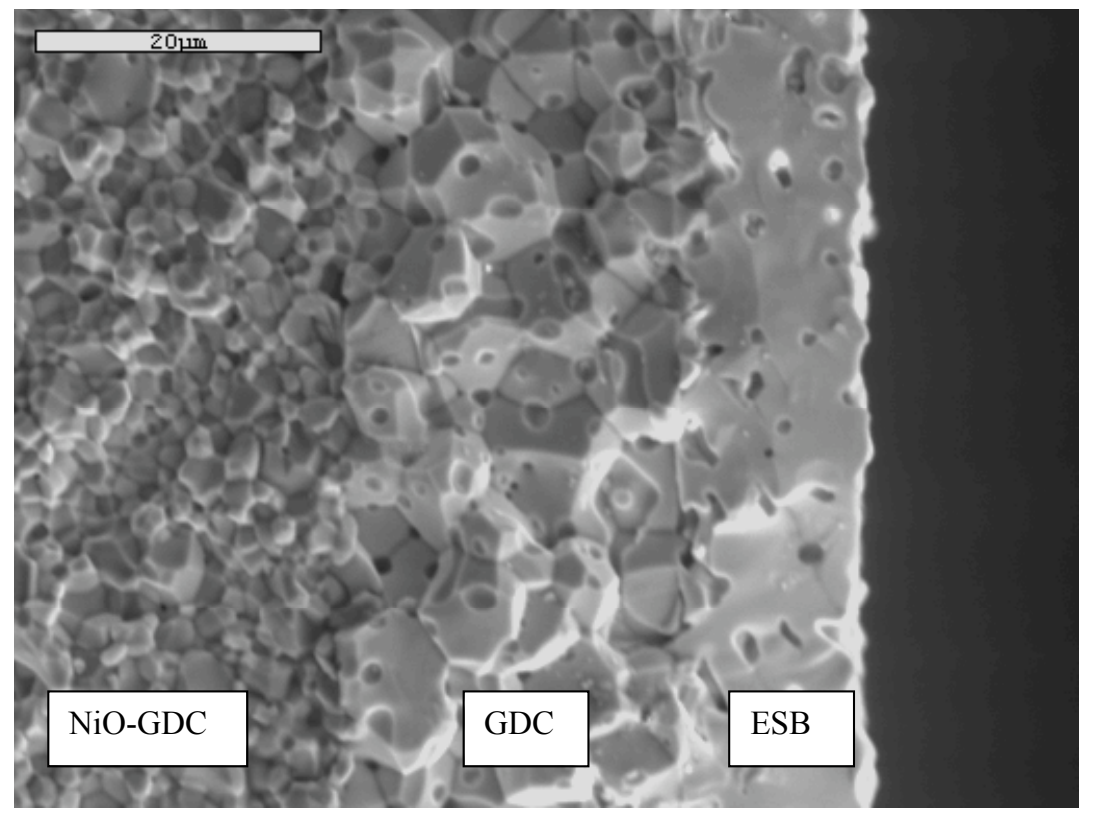

Figure 4.4.10. SEM of Ni-GDC/(GDC $\backslash E S B)$ Anode/(electrolyte) architecture.

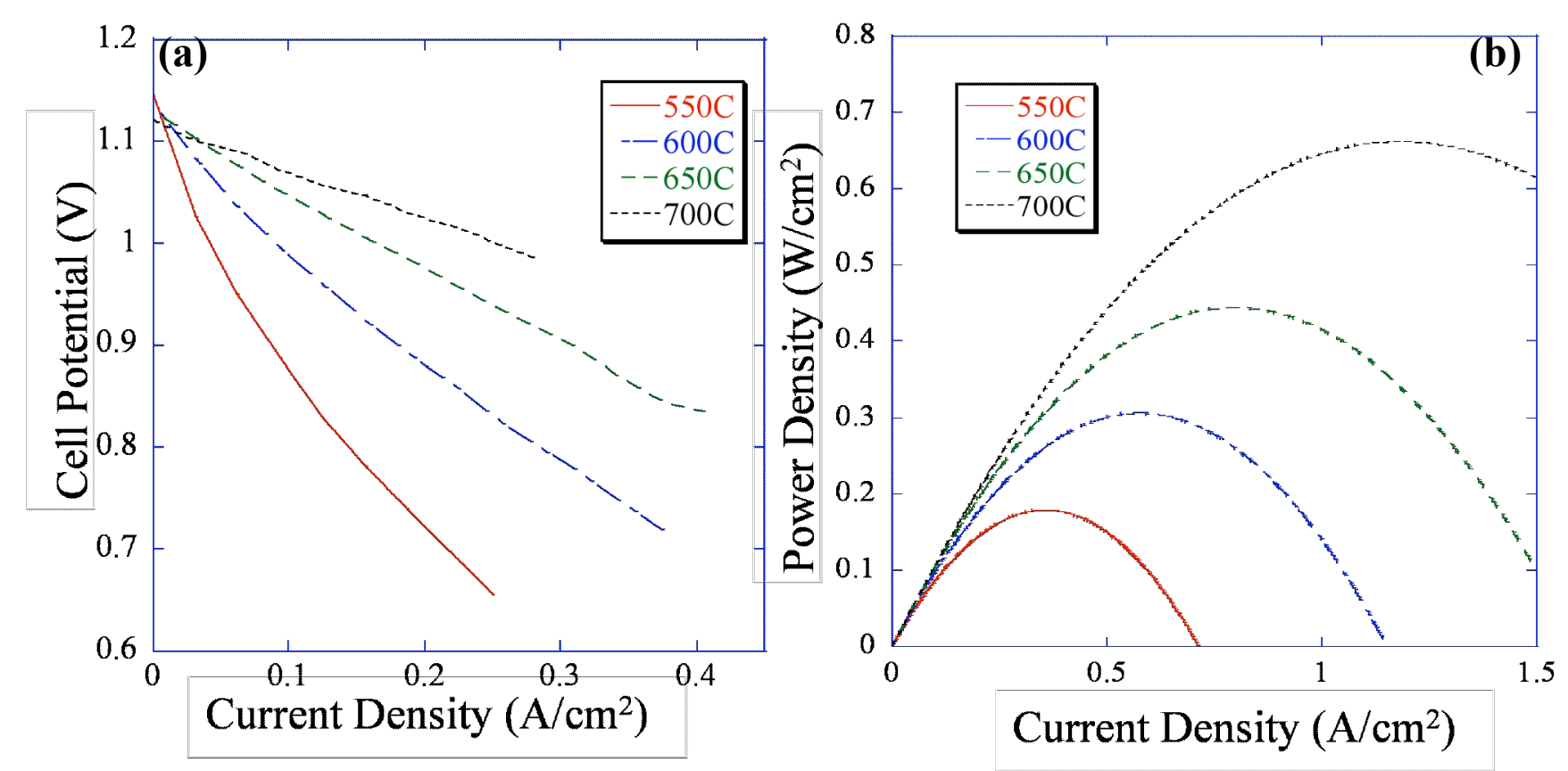

Figure 4.4.11. Projected (a) I-V characteristics and (b) power density of fuel cell with thin film GDC $\backslash E S B$ electrolyte as a function of current density and temperature. 
would have resulted in even higher performance). The results are plotted in Fig. 4.4.11, which shows that just by increasing the OCP, the power density at $700{ }^{\circ} \mathrm{C}$ is now $0.68 \mathrm{~W} / \mathrm{cm}^{2}$, almost double that obtained in Fig. $4.4 .9\left(0.37 \mathrm{~W} / \mathrm{cm}^{2}\right)$. Moreover, Fig. 4.4.11 shows that an acceptable maximum power density of $\sim 0.18 \mathrm{~W} / \mathrm{cm}^{2}$ can be obtained at the relatively low temperature of $550{ }^{\circ} \mathrm{C}$. This is a promising outcome and augurs well for the future of ceria/bismuth oxide bilayers if more research is done to optimize the thickness ratio of the layers, the fabrication of the bilayer and the development of a suitable cathode. Particularly since the ASR in our cells is completely dominated by our unoptimized cathodes.

In the near term, if suitable funding is procured, we plan to study bilayer combinations with a broader range of relative thickness by using anode supported cells under fuel cell conditions to determine optimum value of relative thickness using I-V characteristics as a function of temperature. Cells would be fabricated and characterized as described above. 


\subsection{Cathode Development}

In this section we discuss the development of suitable high performance cathodes for bilayered electrolytes. The suitability of the cathodes is dependent on the stability of the cathode in contact with the ESB layer of the bilayer. The main parameters determining the performance level of the cathode include its catalytic activity, (electronic and ionic) conductivity and structure/morphology all of which contribute to its polarization.

\subsubsection{Fabrication and Characterization}

\section{Ag-ESB Composites}

In order to start with a known cathode that should be stable with the ESB side of our bilayer electrolyte, dual phase composites of Ag and ESB were examined. The polarization resistance for the porous Ag-ESB cathodes as a function of vol.\% Ag was evaluated by Doshi et al [120] and found to exhibit a minimum between 45 and 55 vol.\%. Ag powder (0.6 - 2.0 micron particle size) was obtained from Alfa Aesar®. $\operatorname{Er}_{0.2} \mathrm{Bi}_{0.8} \mathrm{O}_{1.5}$ powder was prepared by a conventional ceramic method by combining appropriate amounts of erbium oxide powder and bismuth oxide powder. The combination was ball milled in ethanol using zirconia milling media for 24 hours and dried in air. The resulting powder was then crushed with a mortar and pestle, fine particles were separated through a 325 mesh screen. Calcining was performed at $850^{\circ} \mathrm{C}$ for 10 hours in order to form the appropriate doped-fluorite structure. XRD was performed on this calcined powder to verify that a stable fluorite structure had formed, see Figs. 3.2, 3.3 and 3.5. Ag and ESB powders were combined on a 1:1 volume percent ratio, mixed in a mortar and pestle using, as organic vehicles, polyvinyl butyral (binder, 3 wt.\%), dibutyl phthalate (plasticizer), and $\alpha$-terpinol (solvent). The slurry was mixed to an appropriate viscosity and screen printed onto each side of an ESB electrolyte substrate. The coated samples were fired at 800 ${ }^{\circ} \mathrm{C}$. Ag mesh and leads were attached using Ag paste to each electrode. The starting materials for powder synthesis and slurry development are summarized in Tables 4.5-1 and 4.5-2.

To test the influence of porosity on electrical performance, 10 vol.\% cellulose powder was added to the slurry during the mixing stage. To test the influence of the ionic conducting phase particle size, ESB powders were also prepared by the citrate process using stoichiometric amounts of hydrated nitrate salts $\mathrm{Bi}\left(\mathrm{NO}_{3}\right)_{3}-\mathrm{X} \cdot \mathrm{H}_{2} \mathrm{O}$ and $\mathrm{Er}\left(\mathrm{NO}_{3}\right)_{3}-5 \cdot \mathrm{H}_{2} \mathrm{O}$. The reactants were dissolved in de-ionized (DI) 
Table 4.5-1. Starting materials used for the synthesis of LSM, GSM, GLSM (GDC+LSM), GGSM $(\mathrm{GDC}+\mathrm{GSM}), \mathrm{LSCF}, \mathrm{ESB}, \mathrm{SDC}$, and GDC powers by citrate process (CP)

\begin{tabular}{ccc}
\hline Starting materials & Purity $(\%)$ & Makers \\
\hline $\mathrm{Ce}\left(\mathrm{NO}_{3}\right)_{3} \cdot 6 \mathrm{H}_{2} \mathrm{O}$ & 99.99 & Kanto Chemical \\
$\mathrm{Sm}\left(\mathrm{NO}_{3}\right)_{3} \cdot 6 \mathrm{H}_{2} \mathrm{O}$ & 99.95 & Kanto Chemical \\
$\mathrm{Gd}\left(\mathrm{NO}_{3}\right)_{3} \cdot 6 \mathrm{H}_{2} \mathrm{O}$ & 99.95 & Kanto Chemical \\
$\mathrm{Er}\left(\mathrm{NO}_{3}\right)_{3} \cdot 5 \mathrm{H}_{2} \mathrm{O}$ & 99.99 & Alfa Aesar \\
$\mathrm{Bi}\left(\mathrm{NO}_{3}\right)_{3} \cdot \mathrm{XH}_{2} \mathrm{O}$ & 99.999 & Alfa Aesar \\
$\mathrm{La}\left(\mathrm{NO}_{3}\right)_{3} \cdot 6 \mathrm{H}_{2} \mathrm{O}$ & 99.99 & Adrich \\
$\mathrm{Sr}\left(\mathrm{NO}_{3}\right)_{2}$ & 99.5 & Kanto Chemical \\
$\mathrm{Mn}\left(\mathrm{NO}_{3}\right)_{3} \cdot \mathrm{XH}_{2} \mathrm{O}$ & 99.99 & Alfa Aesar \\
$\mathrm{Co}\left(\mathrm{NO}_{3}\right)_{2} \cdot 6 \mathrm{H}_{2} \mathrm{O}$ & 98.5 & Alfa Aesar \\
$\mathrm{Fe}\left(\mathrm{NO}_{3}\right)_{3} \cdot \mathrm{XH}_{2} \mathrm{O}$ & 99.999 & Alfa Aesar \\
\hline
\end{tabular}

water in a Pyrex container. Ethylene glycol and citric acid were added as the solvent and the metal chelating agent, respectively. The solution was slowly heated on a hot plate and carefully monitored until the point where the DI water evaporated. At this point the thermo-chemical reaction between nitrate salts occurred, and a precursor foam was produced. The product was calcined at $700{ }^{\circ} \mathrm{C}$ for 2 hrs. A fluorite structure was verified by XRD. SEM revealed that ESB particles prepared by the citrate process (CP) were significantly smaller than those prepared by conventional solid-state synthesis (SSS), see Fig. 3.4. Ag-ESB cathodes were sintered at $800{ }^{\circ} \mathrm{C}$ for 1 hour due to the low melting temperature of silver.

Figure 4.5.1 shows typical surface and cross-sectional images of an Ag-ESB cathode, with the ESB electrolyte. The Ag and ESB particles appear to have sintered well with each other and bonded well at the Ag-ESB/ESB interface. The particles have rounded edges and are smaller in size compared to those of Ag powder.

To test the effect of cathode thickness, Ag-ESB-no cellulose paste (using ESB prepared by the citrate process) was screen-printed to each side of three different ESB pellets using 1 coat on the first pellet, 2 coats on the second pellet, and 4 coats on the third pellet. Analysis of the 1-coat sample showed that the cathode layer was $8 \mu \mathrm{m}$ thick. The cathode thickness (of the multi-coated samples)

Table 4.5-2. Materials used for development of slurry used for screen-printed cathodes.

\begin{tabular}{ccc}
\hline Materials & Function & Amount per gram of powder \\
\hline$\alpha$-terpineol & Solvent & $0.5 \mathrm{cc}$ \\
Dibutyl phtalate & Plastcizer & $0.05 \mathrm{cc}$ \\
Polyvinyl butyral & Binder & $3 \mathrm{wt} . \%$ \\
\hline
\end{tabular}




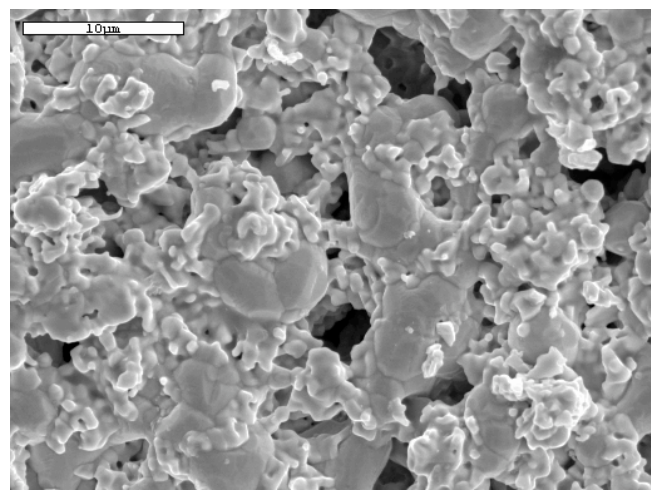

(a) surface images of Ag-ESB

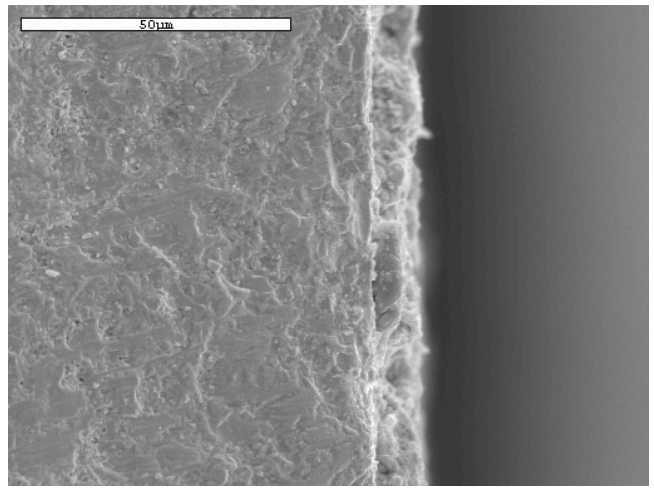

(b) cross-section of Ag-ESB on ESB substrate

Figure 4.5.1. SEM images of Ag-ESB composite cathode.

should be directly proportional to the number of coats, but this has not yet been confirmed. Electrical characterization of these Ag-ESB composite cathodes was performed by impedance spectroscopy. Impedance measurements were monitored with a Solatron SI 1260 impedance analyzer using frequencies ranging from $0.1 \mathrm{~Hz}$ to $10 \mathrm{MHz}$ at temperatures ranging from $400{ }^{\circ} \mathrm{C}$ to $700{ }^{\circ} \mathrm{C}$ in air (30ccm flow rate).

\section{LSM, LSCF and GSM}

Other cathodes were also investigated. $\mathrm{La}_{0.85} \mathrm{Sr}_{0.15} \mathrm{MnO}_{3}$ (LSM), $\mathrm{La}_{0.8} \mathrm{Sr}_{0.2} \mathrm{Co}_{0.8} \mathrm{Fe}_{0.2} \mathrm{O}_{3}$ (LSCF), $\mathrm{Gd}_{0.8} \mathrm{Sr}_{0.2} \mathrm{MnO}(\mathrm{GSM})$, were prepared using the citrate method described above and calcined at $700{ }^{\circ} \mathrm{C}$ for $4 \mathrm{hrs}$. All these powders were milled for $24 \mathrm{hrs}$ in ethanol, using zirconia media, to form cathode inks. The slurries were mixed in a mortar and pestle until an appropriate viscosity was reached then screen-printed onto the bilayer GDC $\backslash$ ESB and SDC $\backslash$ ESB electrolytes, and fired at $900{ }^{\circ} \mathrm{C}$ for 1 hour. The starting materials for powder synthesis and slurry development are summarized in Tables 4.5-1 and $4.5-2$.

The XRD patterns of the LSM and LSCF powders in Fig. 4.5.2 show they have a perovskite single-phase structure. Figure 4.5.2 further shows that the composites GDC+LSM and GDC+GSM have both fluorite and perovskite structures present.

Figure 4.5.3 shows SEM images of LSM, GSM, and composite GDC+LSM cathodes. The particle size and porosity of LSM particles appear smaller than that of the GDC and GDC+LSM, but the shapes of the particles are more spherical in the GSM and GDC+LSM. This observation provides support for the idea that the irregularly shaped particles in the GSM and GDC+LSM cathodes were GDC. The 


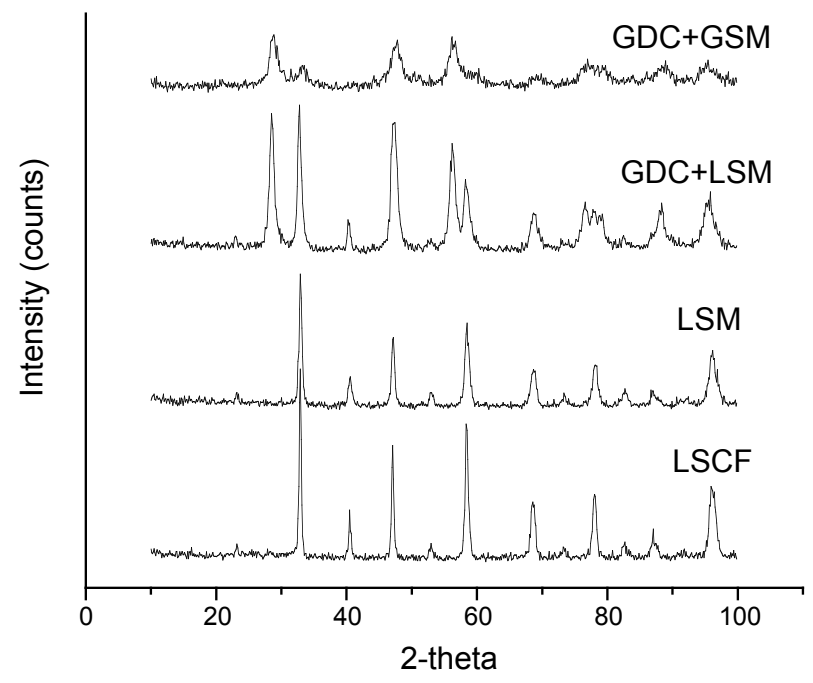

Figure 4.5.2. XRD patterns of calcined LSM, GDC+LSM, GDC+GSM, and LSCF powders fabricated by $\mathrm{CP}$.

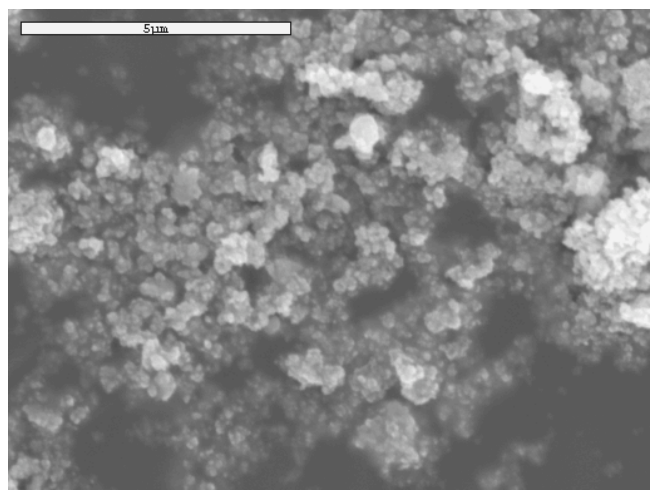

(a) LSM

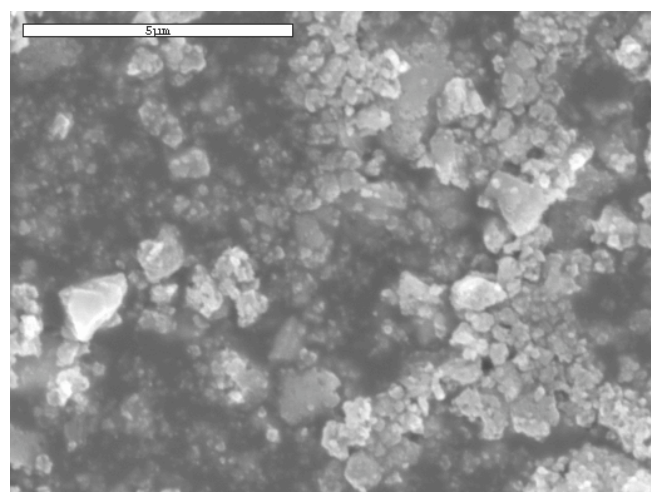

(b) GSM

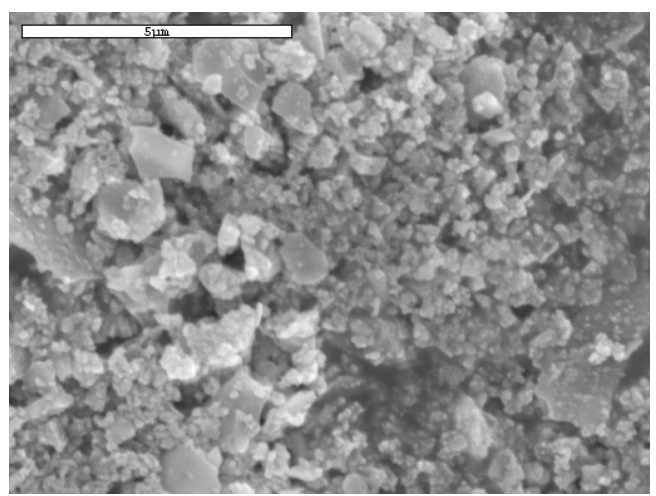

(c) GDC+LSM

Figure 4.5.3. SEM micrograph of LSM, GSM, GDC+LSM cathode materials 
Table 4.5-3. Specific surface area and pore volume and diameter of cathode precursors

\begin{tabular}{ccccccc}
\hline Materials & \multicolumn{3}{c}{ BET $\left(\mathbf{m}^{\mathbf{2} / \mathbf{g})}\right.$} & \multicolumn{3}{c}{$\begin{array}{c}\text { Pore Volume } \\
(\mathbf{c c} / \mathbf{g})\end{array}$} \\
& $\begin{array}{c}\text { Multipoint } \\
\text { BET }\end{array}$ & $\begin{array}{c}\text { Langmuir } \\
\text { method }\end{array}$ & $\begin{array}{c}\text { BJH } \\
\text { method }\end{array}$ & Total pore volume & $\begin{array}{c}\text { Average } \\
\text { pore dia. }\end{array}$ & $\begin{array}{c}\text { BJH } \\
\text { method }\end{array}$ \\
\hline LSM (CP) & 19.0 & 33.0 & 19.1 & 0.258 & 543.3 & 18.9 \\
GDC+LSM & 37.5 & 65.4 & 37.9 & 0.187 & 199.3 & 38.2 \\
GSM (CP) & 47.8 & 84.3 & 52.1 & 0.324 & 270.6 & 95.8 \\
\hline
\end{tabular}

average particle size of the powders, measured by TEM micrographs and with a particle size analyzer, was $20-50 \mathrm{~nm}$ as shown in Table 4.5-3.

\section{Bismuth Ruthenate}

Bismuth ruthenate $\left(\mathrm{Bi}_{2} \mathrm{Ru}_{2} \mathrm{O}_{7}\right)$ was also investigated as a cathodic material for SOFCs because of its catalytic activity and potential compatibility with bismuth oxide, the electrolyte material to which it would be applied in the ceria/bismuth oxide bilayer.

Bismuth ruthenate was produced through conventional solid-state synthesis. Bismuth oxide powder was mixed with ruthenium oxide powder in an amount such that there are equal numbers of each cation. Since only small quantities were made, a mortar and pestle was used to mix the powders. The as mixed powder was then calcined at $975{ }^{\circ} \mathrm{C}$ for two hours. A single-phase pyrochlore structure was obtained from XRD analysis, with no peaks for the original bismuth or ruthenium oxides detected.

Bismuth ruthenate samples were prepared through conventional solid-state synthesis as described in section 4.2 and sintered at $900{ }^{\circ} \mathrm{C}$. In addition, bismuth ruthenate powder was used to prepare a slurry, as described above (for other cathode materials) that was screen-printed onto an ESB substrate. After sintering at $900{ }^{\circ} \mathrm{C}$, this combination was analyzed through AC-impedance spectroscopy.

\subsubsection{Results and Discussion}

$A g-E S B$ and $L S C F$

After sintering of cathode materials, on ESB disks of diameter $11 \mathrm{~mm}, \mathrm{Ag}$ wires were attached to the electrodes using Ag paint, dried and sintered at $800^{\circ} \mathrm{C}$ to ensure good contact between cathodes and current collectors.

Plots of cathode resistance as a function of temperature are shown in Figs. 4.5.4 - 4.5.6. Note that in these figures, the subscripts "SSS" and "CP" refer to ESB powders prepared by the solid-state 


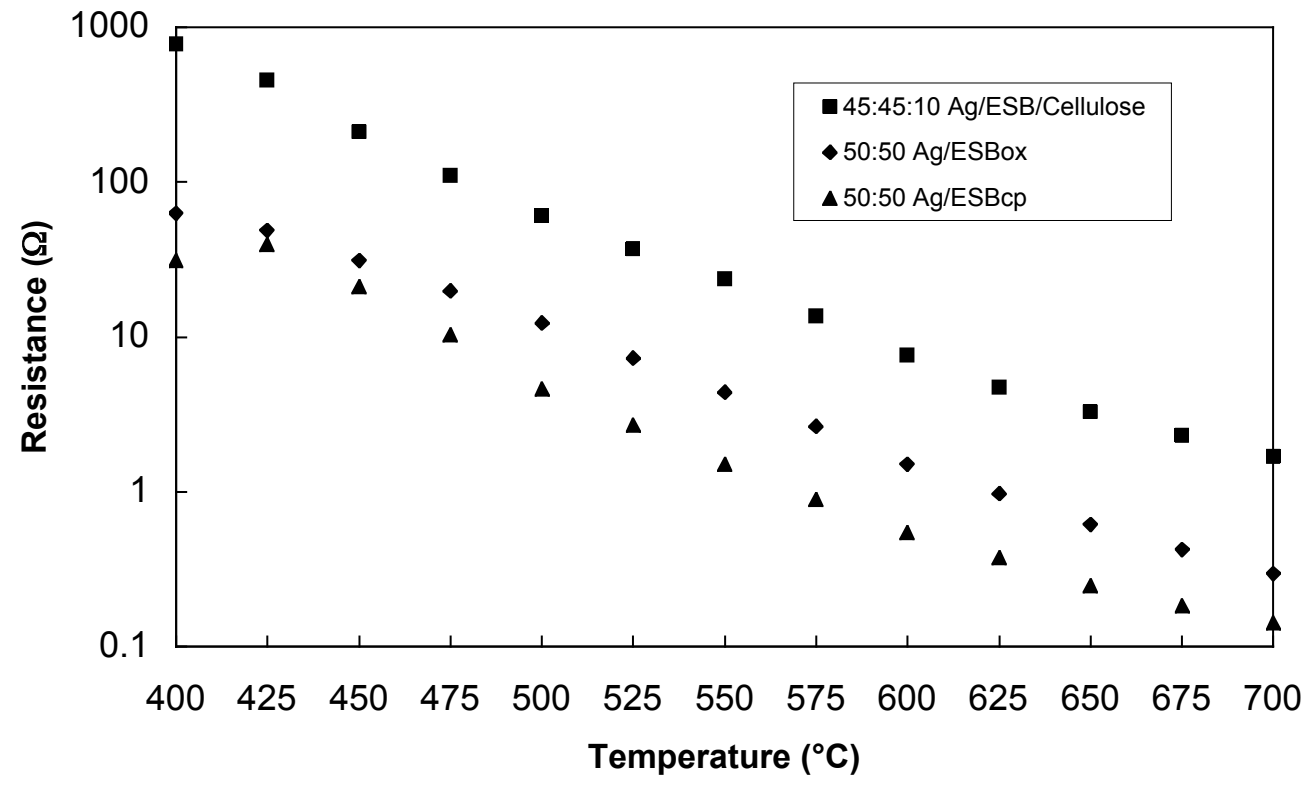

Figure 4.5.4. Comparison of electrical resistance as a function of temperature for 45:45:10 $\mathrm{Ag} / \mathrm{ESB}_{\mathrm{CP}} /$ Cellulose, 50:50 Ag/ESB $\mathrm{ESS}_{\mathrm{SS}}$ and 50:50 Ag/ESB $\mathrm{EP}_{\mathrm{CP}}$ cathodes.

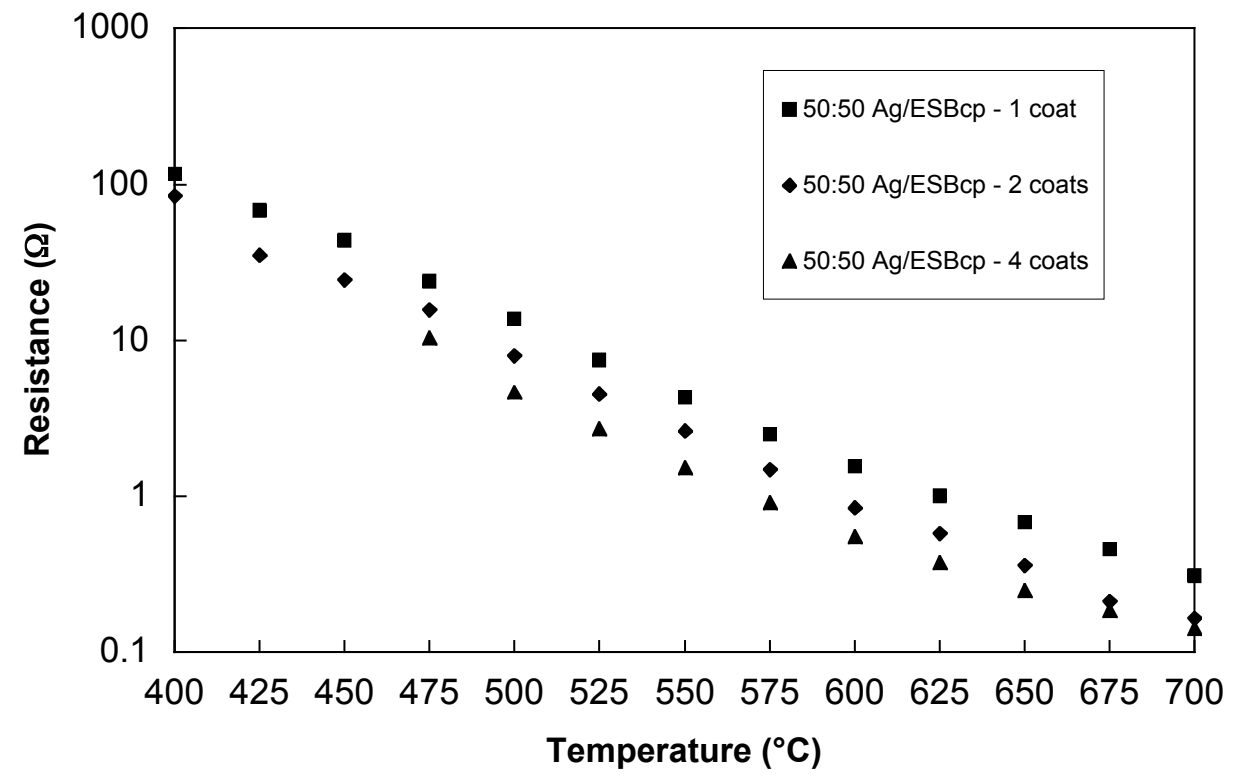

Figure 4.5.5. Electrical resistance as a function of temperature and coating layers for $50: 50 \mathrm{Ag} / \mathrm{ESB}_{\mathrm{CP}}$ cathodes. 


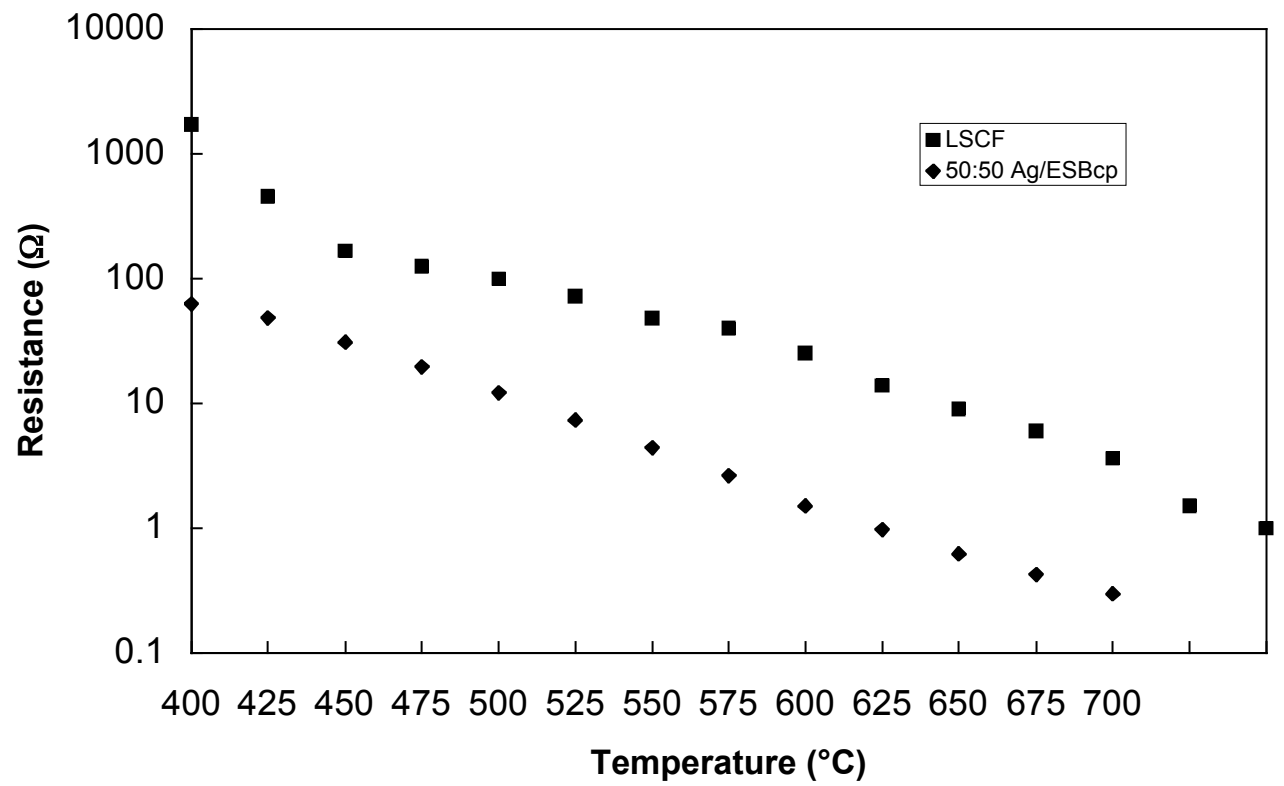

Figure 4.5.6. Comparison of electrical resistance as a function of temperature for $50: 50 \mathrm{Ag} / \mathrm{ESB}_{\mathrm{CP}}$ and LSCF cathodes.

conventional method and from the citrate process, respectively. As expected, reduced particle size of the ESB phase led to a reduction in total electrical resistance (Fig. 4.5.4). Resistance values were also reduced by an increase in cathode thickness (Fig. 4.5.5). The cathodes containing $10 \%$ cellulose pore former showed resistance that was an order of magnitude larger than those containing no pore former, Fig. 4.5.4. This result could be attributed to the inherent porosity of the Ag-ESB-no cellulose cathode slurry. The additional 10 vol.\% of porosity could reduce the connectivity of both Ag and ESB phases, resulting in the poor performance observed for thin cathodes. The porosities of these cathodes will be examined by SEM in the near future to verify this point. Obviously we have not reached the optimum thickness for the cathodes as Fig. 4.5.5 indicates that better performance should be attained with a thicker cathode.

Finally, the LSCF cathode did not perform as well as the Ag-ESB cathodes, however, its porosity was not optimized and we anticipate improved performance from both, as more work is done in this area. Figure 4.5.6 shows that the LSCF cathode is consistently outperformed by the $\mathrm{Ag} / \mathrm{ESB}_{\mathrm{CP}}$ cathode.

\section{Bismuth Ruthenate}

Thus far it has proven difficult to obtain conductivity data for bismuth ruthenate due to it's extremely high conductivity. The impedance of all of the samples prepared were too low to measure 
with the Solartron impedance analyzer. Similarly, bismuth ruthenate cathodes on ESB disks had too low of an impedance to accurately measure. Four-point DC conductivity tests will be made on a bar of bismuth ruthenate in the near future. 


\subsection{Defect Transport in a Single Layer Electrolyte}

In order to model the performance and stability of a bilayer electrolyte we first must have an accurate description of defect transport in a single solid oxide layer. Isobaric transport properties have been extensively studied and are well defined. However, a widely accepted, and accurate, formalism for the transport properties of mixed ionic-electronic conductors (MIECs) in oxygen partial pressure, $P_{\mathrm{O}_{2}}$, gradients has yet to emerge. This arises from the difficulty in obtaining assumption-free solutions to the transport laws describing the systems, due primarily to the presence of gradients in the defect concentrations and their dependence (in the bulk and at the boundaries) on the operating conditions of the devices or components $[121,125]$.

Previous researchers [122 - 124] used fixed boundary values for the defect concentrations and linear potential distributions. Fixed boundary values are independent of an external (output or applied) potential, $\Phi_{\text {ext }}$. This implies that $\Phi_{\text {ext }}$ only affects the spatial distribution of defects inside the MIEC. In principle, this is not possible, since the activities of all the reacting chemical species cannot be held constant while changing the potential at the interface $[125,126]$. Still, in some limiting conditions it may be a reasonable approximation, so the nature of these conditions and the impact made by the misuse of this assumption is discussed. Similarly, the assumption of a linear potential distribution inside an MIEC, in a $P_{\mathrm{O}_{2}}$ gradient, bears careful analysis.

In this work, we derive expressions for defect transport in $P_{\mathrm{O}_{2}}$ gradients that include potentialdependent boundary conditions and allow non-linear potential distributions. Our discussion is restricted to $n$-type, acceptor-doped, oxide MIECs where oxygen vacancies and electrons are the only mobile defects (e.g., gadolinia- or samaria-doped ceria). In typical $P_{\mathrm{O}_{2}}$ 's $\left(10^{-25}\right.$ atm to $\left.1 \mathrm{~atm}\right)$, the concentrations of other defects are negligible [127 - 129] for these materials.

\subsubsection{General Equations}

Definitions, laws and basic relations used in the derivation of the models are summarized in this section. Broadly speaking, these relations fall into three categories: fundamental equations of general applicability to all MIECs, equations arising from the solid oxide fuel cell (SOFC) or electrocatalytic reactor (ECR) operation and equations specific to the material used as the case study application for the models, acceptor-doped ceria in this case. 


\section{Laws and Definitions}

In oxide MIECs, defect transport of the $i$ th defect is described by the Nernst-Planck flux equation

$$
j_{i}=-D_{i} \nabla c_{i}-u_{i} c_{i} \nabla \phi=-z_{i}^{-2} q^{-2} \sigma_{i} \nabla \tilde{\mu}_{i}
$$

and the current density equation:

$$
J=\sum_{i} J_{i}=q \sum_{i} z_{i} j_{i}
$$

where

$$
\sigma_{i}=z_{i} q u_{i} c_{i}=z_{i}^{2} q^{2} D_{i} k_{B}^{-1} T^{-1} c_{i}
$$

and

$$
\tilde{\mu}_{i}=\mu_{i}+z_{i} q \phi=\mu_{i}^{\circ}+k_{B} T \ln c_{i}+z_{i} q \phi
$$

$j$ is flux density, $J$ is current density, $D$ is diffusivity, $c$ is concentration, $u$ is electrical mobility, $\phi$ is the Galvani potential, $z$ is charge equivalence, $q$ is the elementary electronic charge, $\sigma$ is conductivity, $\tilde{\mu}$ is the electrochemical potential, $\mu_{i}^{\circ}$ is the chemical potential of the $i$ th defect in the standard state, $\mu_{i}$ is the chemical potential of the $i$ th defect, $k_{B}$ is Boltzman's constant, $T$ is temperature and the subscript " $i$ " refers to the $i$ th defect species. If all chemical reactions take place at the electrode surfaces, then, in steady state conditions, $\nabla j_{i}=0-$ i.e., $j_{i}$ and $J$ are independent of position.

Two additional transport properties of interest are the total conductivity, $\sigma_{t o t}$, and the transference number, $t_{i}$, which are defined as

$$
\sigma_{t o t}=\sum_{i} \sigma_{i}
$$

and

$$
t_{i}=\sigma_{i} / \sigma_{t o t}
$$

respectively. Additionally, if concentration gradients exist in the MIEC, then the $i$ th defect resistance, $R_{i}$, by definition an average property, is given by [122 - 124]

$$
R_{i}^{-1}=\bar{\sigma}_{i} S L^{-1}
$$


where $\bar{\sigma}_{i}$ is the average conductivity, $S$ is the cross-sectional area and $L$ the thickness of the MIEC. Accordingly, $\bar{\sigma}_{i}$ is given by

$$
\bar{\sigma}_{i}^{-1}=L^{-1} \int_{0}^{L}\left[\sigma_{i}(x)\right]^{-1} \cdot \mathrm{d} x
$$

which, when combined with Eqs. (4.6.1), (4.6.3) and (4.6.4), yields

$$
\bar{\sigma}_{i}^{-1}=-\frac{k_{B} T}{z_{i}^{2} q^{2} j_{i} L}\left(\int_{c_{i_{0}}}^{c_{i_{L}}} c_{i}^{-1} \cdot \mathrm{d} c_{i}+\frac{z_{i} q}{k_{B} T} \int_{\phi_{0}}^{\phi_{L}} \mathrm{~d} \phi\right)=-\frac{k_{B} T}{z_{i}^{2} q^{2} j_{i} L}\left(\ln \frac{c_{i_{L}}}{c_{i_{0}}}+\frac{z_{i} q}{k_{B} T} \Delta \phi\right)
$$

where $x$ is the positional variable $(0 \leq x \leq L)$. Accordingly, the total average conductivity, $\bar{\sigma}_{t o t}$, and the average transference number, $\bar{t}_{i}$, are given by

$$
\bar{\sigma}_{t o t}=\sum_{i} \bar{\sigma}_{i}
$$

and

$$
\bar{t}_{i}=\bar{\sigma}_{i} / \bar{\sigma}_{t o t}
$$

respectively.

\section{Charge Neutrality}

We now introduce the local charge neutrality approximation,

$$
\sum_{i} z_{i} c_{i} \approx 0
$$

which is used because it simplifies the mathematics and electrochemical systems generally show little deviation from it [125]. We realize that the potential distribution inside an MIEC is best described by Poisson's equation, which is given by

$$
\nabla^{2} \phi=\frac{q}{\varepsilon} \sum_{i} z_{i} c_{i}
$$

where $\varepsilon$ is permittivity. However, as Newman points out [125], the local charge neutrality approximation remains valid and does not require the potential distribution to be Laplacian (i.e., $\nabla^{2} \phi=$ $0)$ since the term $q / \varepsilon$ is usually very large.

For typical $n$-type MIECs, the charge neutrality approximation is written as

$$
z_{V} c_{V}+z_{A} c_{A}+z_{e} c_{e} \approx 0
$$


where the subscripts " $V$ ", " $e$ " and " $A$ " refer to oxygen vacancies, electrons and a singly charged acceptor dopant, respectively; $c_{A}$ is a constant. Also, $z_{A}=-1, z_{e}=-1$ and $z_{V}=2$.

\section{Current-Voltage Relationships}

The equivalent circuit of an SOFC/ECR is shown in Fig. 4.6.1. Electrodes are excluded and $P_{\mathrm{O}_{2}, 0}$ and $P_{\mathrm{O}_{2}, L}$ are the effective $P_{\mathrm{O}_{2}}$ 's on either side of the MIEC. Analysis of the equivalent circuit yields

$$
J=J_{V}+J_{e}=\left[\left(\Phi_{t h}-\Phi_{e x t}\right) R_{V}^{-1}-\Phi_{e x t} R_{e}^{-1}\right] S^{-1}
$$

where $\Phi_{\text {ext }}$ is the potential difference across a load ( $\mathrm{s}_{1}$ open and $\mathrm{s}_{2}$ closed) or applied by an external source ( $\mathrm{s}_{1}$ closed and $\mathrm{s}_{2}$ open); $\Phi_{t h}$ is the theoretical (Nernst) voltage and the subscripts have their usual meaning. Combining Eqs. (4.6.7), (4.6.10) and (4.6.13), yields

$$
J=\left(\bar{t}_{i o n} \Phi_{t h}-\Phi_{e x t}\right) \bar{\sigma}_{t o t} L^{-1}=\eta \bar{\sigma}_{t o t} L^{-1}
$$

where $\eta$ is the overpotential [125] and

$$
\eta=\bar{t}_{i o n} \Phi_{t h}-\Phi_{e x t}
$$

In open-circuit conditions (both $\mathrm{s}_{1}$ and $\mathrm{s}_{2}$ open, see Fig. 4.6.1),

$$
J=0, \eta=0 \text { and } \Phi_{e x t}=\Phi_{o c}
$$

where $\Phi_{o c}$ is the open-circuit voltage. Therefore, from Eq. (4.6.15),

$$
\bar{t}_{i o n}=\Phi_{o c} / \Phi_{t h}
$$

In short-circuit conditions ( $\mathrm{s}_{1}$ open and $\mathrm{s}_{2}$ closed, see Fig. 4.6.1)

$$
R_{L}=0, \Phi_{e x t} \approx 0 \text { and } \eta \approx \Phi_{t h}
$$

where $R_{L}$ is the load resistance. In short-circuit conditions $J_{e}$ is at its minimum since the external circuit is at it most conductive $\left(R_{L}=0\right)$.

In forward and reverse bias ( $\mathrm{s}_{1}$ closed and $\mathrm{s}_{2}$ open, see Fig. 4.6.1),

$$
\Phi_{e x t}<0 \text { and } \eta>\bar{t}_{i o n} \Phi_{t h}
$$

and

$$
\Phi_{e x t}>\bar{t}_{i o n} \Phi_{t h} \text { and } \eta<0
$$




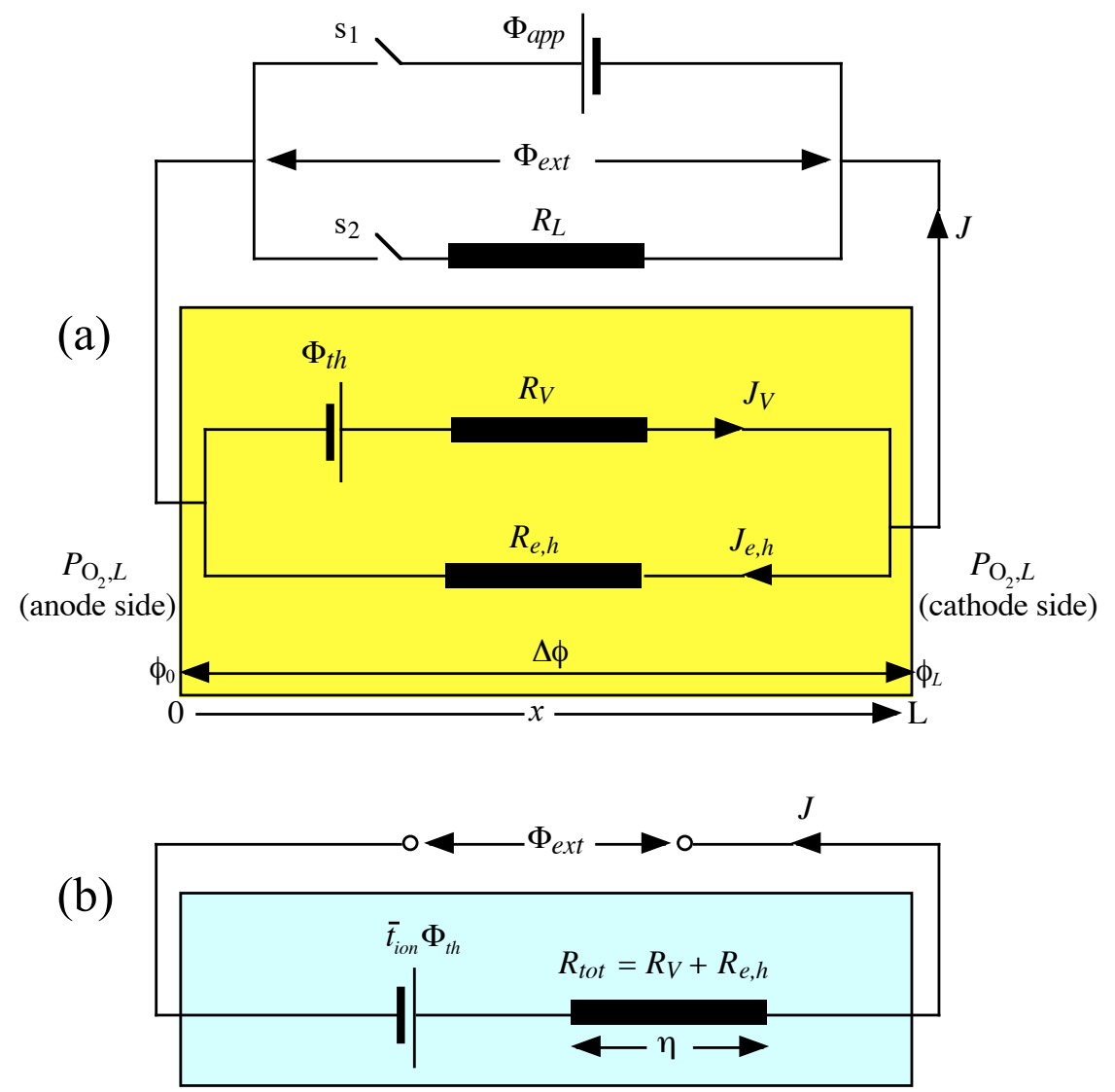

Figure 4.6.1. Equivalent circuit diagrams of an SOFC ( $\mathrm{s}_{1}$ open, $\mathrm{s}_{2}$ closed) or an ECR ( $\mathrm{s}_{1}$ closed, $\mathrm{s}_{2}$ open) showing relationships between (a) the currents and (b) the voltages.

respectively. In both forward and reverse bias, $\Phi_{\text {ext }}$ is the voltage from a power supply (i.e., $\Phi_{\text {ext }}=$ $\Phi_{a p p}$, see Fig. 4.6.1) rather than across a load.

Finally, in fuel cell conditions ( $\mathrm{s}_{1}$ open and $\mathrm{s}_{2}$ closed, see Fig. 4.6.1)

$$
0<\Phi_{e x t}<\bar{t}_{i o n} \Phi_{t h} \text { and } 0<\eta<\Phi_{t h}
$$

\section{Local Equilibrium Approximation}

The equilibrium oxygen exchange reaction between the MIEC and the gas phase, in Kröger-Vink notation, is given by

$$
\mathrm{O}_{\mathrm{O}}^{\times} \Leftrightarrow \mathrm{V}_{\mathrm{O}}^{\ddot{*}}+2 e^{\prime}+\frac{1}{2} \mathrm{O}_{2} \quad K_{r}=c_{V} c_{e}^{2} P_{\mathrm{O}_{2}}^{\frac{1}{2}}
$$

where $K_{r}$ is the equilibrium (mass action) constant for the reaction. Therefore if local equilibrium is attained in the system [122 - 125], then

$$
-\frac{1}{2}\left(\mu_{\mathrm{O}_{2}, L}-\mu_{\mathrm{O}_{2}, 0}\right)=\tilde{\mu}_{V_{L}}-\tilde{\mu}_{V_{0}}+z_{V}\left(\tilde{\mu}_{e_{L}}-\tilde{\mu}_{e_{0}}\right)
$$


However, by definition,

$$
\begin{gathered}
\tilde{\mu}_{e_{L}}-\tilde{\mu}_{e_{0}}=-q \Phi_{e x t} \\
\mu_{\mathrm{O}_{2}, L}-\mu_{\mathrm{O}_{2}, 0}=k_{B} T \ln \frac{P_{\mathrm{O}_{2}, L}}{P_{\mathrm{O}_{2}, 0}}=2 z_{V} \Phi_{t h} \quad \text { (the Nernst Equation) }
\end{gathered}
$$

and

$$
\tilde{\mu}_{V_{L}}-\tilde{\mu}_{V_{0}}=k_{B} T \ln \left(c_{V_{L}} / c_{V_{0}}\right)+z_{V} q\left(\phi_{L}-\phi_{0}\right)
$$

Therefore, by substituting the definitions in Eqs. (4.6.18) - (4.6.20) into Eq. (4.6.17), it may be rewritten as:

$$
\phi_{L}-\phi_{0}=\Delta \phi=\Phi_{e x t}-\Phi_{t h}-\frac{k_{B} T}{z_{V} q} \ln \frac{c_{V_{L}}}{c_{V_{0}}}
$$

\section{Boundary Conditions}

For MIECs, the defect concentration boundary values are set by a combination of the charge balance, Eq. (4.6.12), and defect equilibria [127 - 129]. Relations for the functional dependence of $c_{V}$ and $c_{e}$ on $P_{\mathrm{O}_{2}}$, obtained from a recent model $[127,128]$, are given by

$$
c_{V}\left(P_{\mathrm{O}_{2}}\right)=\left[\frac{3}{4} K_{r}^{\frac{1}{2}} c_{O}^{\frac{1}{2}} c_{M} P_{\mathrm{O}_{2}}^{-\frac{1}{4}}+\left(\frac{1}{2} c_{A}\right)^{\frac{3}{2}}\right]^{\frac{2}{3}}
$$

and

$$
c_{e}\left(P_{\mathrm{O}_{2}}\right)=K_{r}^{\frac{1}{2}} c_{O}^{\frac{1}{2}} c_{M} P_{\mathrm{O}_{2}}^{-\frac{1}{4}}\left[\frac{3}{4} K_{r}^{\frac{1}{2}} c_{O}^{\frac{1}{2}} c_{M} P_{\mathrm{O}_{2}}^{-\frac{1}{4}}+\left(\frac{1}{2} c_{A}\right)^{\frac{3}{2}}\right]^{-\frac{1}{3}}
$$

respectively, where $c_{M}$ and $c_{O}$ are cation and oxygen-ion concentrations, respectively; $K_{r}$ is the equilibrium constant for the oxygen exchange reaction between MIEC and gaseous $\mathrm{O}_{2}$, Eq. (4.6.16). In the dilute case, $c_{M} \gg c_{e}$ and $c_{O}>c_{V}$, so $c_{M}$ and $c_{O}$ are approximately constant. The boundary values, $c_{i_{0}}$, and $c_{i_{L}}$, are had by substituting $P_{\mathrm{O}_{2}, 0}$ and $P_{\mathrm{O}_{2}, L}$ into Eqs. (4.6.22) and (4.6.23).

The total free energy of an electrochemical reaction has a chemical (open-circuit) and an electrical (due to $\eta$ ) part $[125,126]$. Therefore, the free energy for the external equilibrium reaction, $\Delta G_{r}-K_{r}=\exp \left(-\Delta G_{r} / k_{B} T\right)-$ is given by 
Anode: $\quad \Delta G_{r}=\Delta G_{r}^{o c}-q \eta \quad$ Cathode: $\quad \Delta G_{r}=\Delta G_{r}^{o c}+q \eta$

Eq. (4.6.24) is substituted into Eqs. (4.6.22) and (4.6.23) to obtain potential-dependent boundary conditions for the MIEC.

\subsubsection{Linear Potential Model}

In this section, expressions for the spatial distribution and transport of defects in acceptor-doped oxide MIECs are derived by assuming that the potential is Laplacian-i.e., $\nabla^{2} \phi=0$ and the electric field, $\nabla \phi$, is a constant such that $\nabla \phi=\Delta \phi / L=\left(\phi_{L}-\phi_{0}\right) / L$.

Eqs. (4.6.1), (4.6.2) and (4.6.12) are combined, with the result:

$$
-z_{e}\left(D_{e}-D_{V}\right) \nabla c_{e}=z_{e}\left(u_{e}-u_{V}\right) c_{e} \nabla \phi+u_{V} c_{A} \nabla \phi+J q^{-1}
$$

For boundary conditions of $c_{i}=c_{i_{0}}$ at $x=0$ and $c_{i}=c_{i_{L}}$ at $x=L(i=V, e$, or $A)$, Eq. (4.6.25) has a solution that, combined with Eq. (4.6.12), yields:

$$
c_{i}(x)=\frac{c_{i_{L}}-c_{i_{0}} \exp (\beta \Delta \phi)+\left(c_{i_{0}}-c_{i_{L}}\right) \exp (\beta \Delta \phi x / L)}{1-\exp (\beta \Delta \phi)}
$$

where $\beta=-\left(u_{e}-u_{V}\right) /\left(D_{e}-D_{V}\right)$ and $\Delta \phi$ is given by Eq. (4.6.21).

Having obtained, $c_{i}(x)$, all properties that depend on it may now be derived. Combining Eqs. (4.6.1) and (4.6.26), yields for the flux density of electronic defects:

$$
j_{i}(\Delta \phi, x)=-u_{i} \frac{\Delta \phi}{L} \frac{\left(1+k_{B} T \beta / z_{i} q\right)\left(c_{i_{0}}-c_{i_{L}}\right) \exp (\beta \Delta \phi x / L)+c_{i_{L}}-c_{i_{0}} \exp (\beta \Delta \phi)}{1-\exp (\beta \Delta \phi)}
$$

This result shows that $\nabla j_{V} \neq 0$ (i.e., steady state is not achieved), unless $c_{V_{0}} \approx c_{V_{L}}$ (i.e., $\nabla c_{V} \approx 0$ ). Likewise, $\nabla j_{e} \neq 0$ unless $k_{B} T \beta \approx-z_{e} q$ (i.e., $D_{e}>D_{V}$ ). In other words, for $\nabla j_{i}=0$, the linear potential model is applicable only to good electrolytes and/or good electronic conductors. (Other possibilities, namely $c_{e_{0}} \approx c_{e_{L}}$ and $D_{V}>>D_{e}$, are excluded because they are unrealistic for the present context.) This is a consequence of assuming that $\nabla \phi$ is constant throughout the MIEC, i.e., not allowing the electric field to compensate for changes in the defect concentration gradients by changing its value. Nevertheless, if we apply these constraints, Eq. (4.6.27) becomes 


$$
j_{i}(\Delta \phi)=-u_{i} \frac{\Delta \phi}{L} \cdot \frac{c_{i_{L}}-c_{i_{0}} \exp (\beta \Delta \phi)}{1-\exp (\beta \Delta \phi)}
$$

To obtain an expression for the average ionic transference number, $\bar{t}_{i o n}$ (i.e., $\left.\bar{\sigma}_{V} / \bar{\sigma}_{t o t}\right)$, Eq. (4.6.26) (or (4.6.28)) is combined with Eqs. (4.6.3), (4.6.4) and (4.6.8) - (4.6.11) with the result:

$$
\bar{t}_{\text {ion }}(\Delta \phi)=\left[1+\Theta \frac{c_{e_{L}}-c_{e_{0}} \exp (\beta \Delta \phi)}{c_{V_{L}}-c_{V_{0}} \exp (\beta \Delta \phi)} \cdot \frac{\ln \left(c_{V_{0}} / c_{V_{L}}\right)+\beta \Delta \phi}{\ln \left(c_{e_{0}} / c_{e_{L}}\right)+\beta \Delta \phi}\right]^{-1}
$$

where $\Theta=z_{e} u_{e} /\left(z_{V} u_{V}\right)$. Similarly, expressions current efficiency, $\zeta_{J}$, and power efficiency, $\zeta_{P}$, are obtained by combining Eqs. (4.6.2), (4.6.12) and (4.6.28), with the result:

$$
\zeta_{J}(\Delta \phi)=\frac{J}{J_{V}}=1+\Theta \frac{c_{e_{L}}-c_{e_{0}} \exp (\beta \Delta \phi)}{c_{V_{L}}-c_{V_{0}} \exp (\beta \Delta \phi)}
$$

and

$$
\zeta_{P}(\Delta \phi)=\frac{J \Phi_{e x t}}{J_{V} \Phi_{t h}}=\frac{\Phi_{e x t}}{\Phi_{t h}}\left[1+\Theta \frac{c_{e_{L}}-c_{e_{0}} \exp (\beta \Delta \phi)}{c_{V_{L}}-c_{V_{0}} \exp (\beta \Delta \phi)}\right]
$$

\section{Results and Discussion}

We now apply the results of the Linear Potential Model to samaria-doped ceria (SDC), which is a typical $n$-type oxide MIEC. The relevant constants for SDC were obtained from the literature and our previous work $[127,130-132]$. They are, $D_{V} \approx 4.3 \times 10^{-12} \mathrm{~m}^{2} \mathrm{~V}^{-1} \mathrm{~s}^{-1} ; D_{e} \approx 2 \times 10^{-10} \mathrm{~m}^{2} \mathrm{~V}^{-1} \mathrm{~s}^{-1} ; K_{r}(\eta$ $=0) \approx 9.97 \times 10^{71} \mathrm{~m}^{-9} \mathrm{~atm}^{1 / 2}$ and $c_{A}=5 \times 10^{27} \mathrm{~m}^{-3}$. The environmental conditions are $T=1073 \mathrm{~K}, P_{\mathrm{O}_{2}, 0}$ $=10^{-20}$ atm. and $P_{\mathrm{O}_{2}, L}=0.21 \mathrm{~atm}$. Also, in order to use potential-dependent boundary conditions, the system of equations consisting of Eqs. (4.6.15), (4.6.21), (4.6.22), (4.6.23), (4.6.24) and (4.6.29) must be solved first to obtain consistent values for $\eta, \Phi_{\text {ext }}$ and $\bar{t}_{i o n}$, a few of these are listed in Table 4.6-1.

Table 4.6-1. Selected values of $\Phi_{\text {ext }}, \eta, \Delta \phi$, and $\bar{t}_{\text {ion }}$ obtained from numerical solution of the system of equations: Eqs. (4.6.9), (4.6.11), (4.6.12), (4.6.13), (4.6.14) and (4.6.19).

\begin{tabular}{ccccc}
\hline & $\Phi_{e x t} / \Phi_{t h}$ & $\eta / \Phi_{t h}$ & $\Delta \phi / \Phi_{t h}$ & $\bar{t}_{i o n}$ \\
\hline short-circuit $\left(\nabla c_{i}<0\right)$ & 0.0 & 1.0 & -0.9 & 0.99 \\
open-circuit $\left(\nabla c_{i}<0\right)$ & 0.8 & 0.0 & -0.2 & 0.80 \\
reverse bias $\left(\nabla c_{i}=0\right)$ & 2.0 & -1.0 & 1.0 & 0.96 \\
reverse bias $\left(\nabla c_{i}>0\right)$ & 3.0 & -2.0 & 2.0 & 1.00 \\
\hline
\end{tabular}



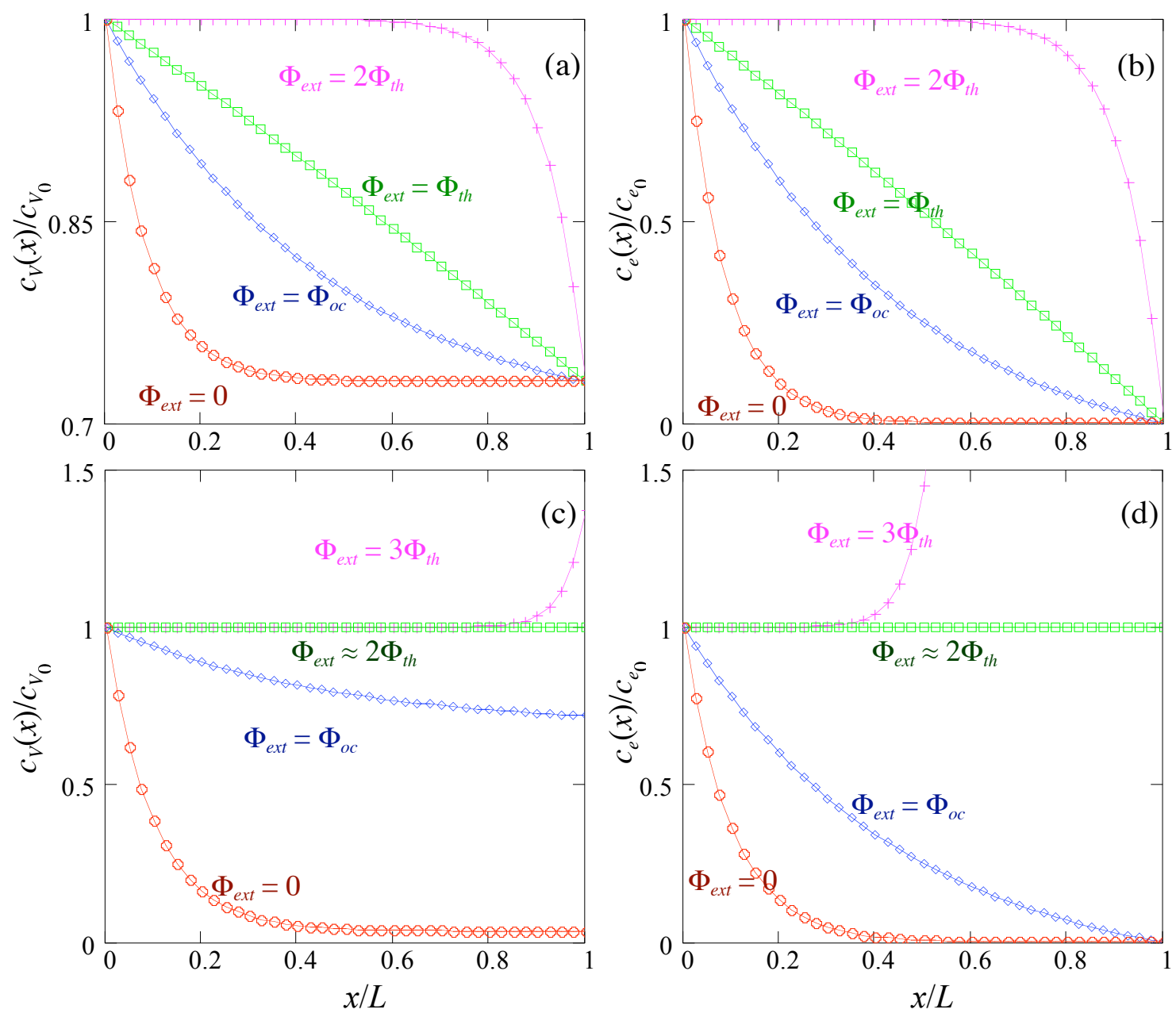

Figure 4.6.2. Distribution of (a) vacancies (fixed boundary values) (b) electrons (fixed) (c) vacancies (potential-dependent) and (d) electrons (potential-dependent).

Figure 4.6.2 shows normalized defect concentration profiles with fixed (Figs. 4.6.2a and 4.6.2b) and with potential-dependent (Figs. 4.6.2c and 4.6.2d) boundary conditions. Figs. 4.6.2a and 4.6.2b are similar to those produced by Riess [122]. It is evident that the electron (i.e., the minority defect) concentration profiles are strongly influenced by the operating conditions, less so for the oxygen vacancies. The influence of $\Phi_{\text {ext }}$ on the boundary values, as well as the internal defect distribution, is seen in Figs. 4.6.2c and 4.6.2d when potential-dependent boundary conditions are used. Notably, one observes that the slopes of the concentration profiles change sign as $\Phi_{e x t}$ passes through the value $2 \Phi_{t h}$. When $\Phi_{e x t}=2 \Phi_{t h}$, the external power source eliminates the chemical potential gradients of the defect species and a flat defect concentration profile observed. In other words, at this point $\Phi_{\text {ext }}$ is large enough to overcome the Nernst potential and has reversed the concentration gradients originally 

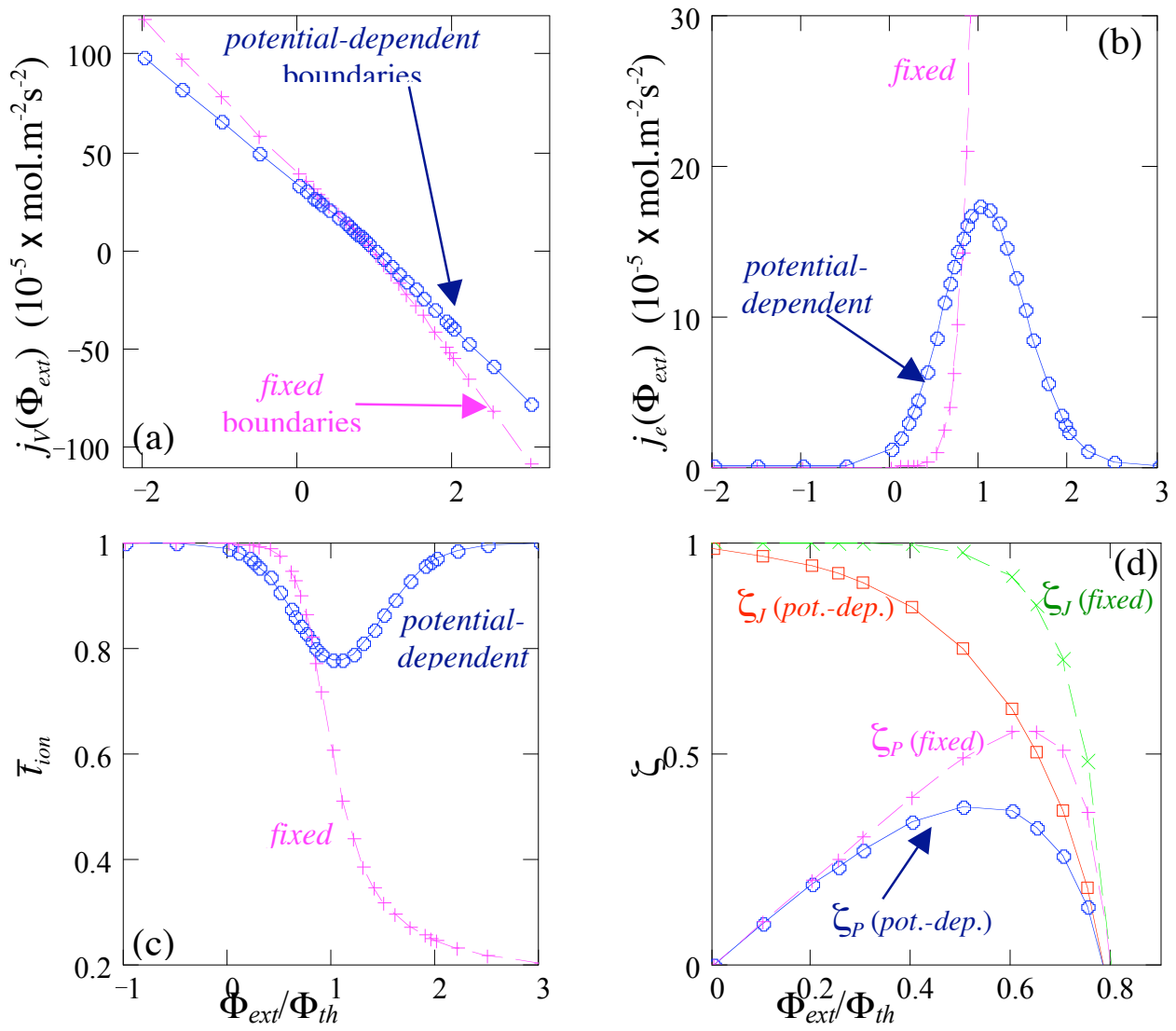

Figure 4.6.3. Effect of using potential-dependent vs. fixed boundary conditions on (a) $j_{V}(\mathrm{~b}) j_{e}$ (c) $\bar{t}_{\text {ion }}$ and (d) $\zeta_{J}$ and $\zeta_{P}$, for $1 \mathrm{~mm}$ thick SDC electrolyte.

imposed by $P_{\mathrm{O}_{2}, 0}$ and $P_{\mathrm{O}_{2}, L}$. This feature is not observed with fixed boundary conditions, which force the concentration gradients to maintain the same sign irrespective of the $\Phi_{\text {ext }}$.

Figures 4.6.3a and 4.6.3b show the dependence of $j_{i}$ on $\Phi_{\text {ext }}$ and compare the effects of using fixed vs. potential-dependent boundary values. Figure 4.6.3a shows an almost linear dependence of $j_{V}$ on $\Phi_{e x t}$ that agrees with the low overpotential approximation of the Butler-Volmer equation (i.e., for $j_{V}$ $>>j_{e}$ ) and is required by a linear (Ohmic) potential [125]. When fixed boundary values are used, $j_{V}$ is overestimated for both $\Phi_{e x t}>\Phi_{o c}$ and $\Phi_{e x t}<\Phi_{o c}$. This is a result of ignoring the influence of $\Phi_{e x t}$ on the boundary values.

Figure 4.6.3b shows that with potential-dependent boundary values, $j_{e}$ approaches a maximum at $\Phi_{e x t}=\Phi_{t h}$, and goes to a minimum as the external circuit grows less resistive. With fixed boundary values, $j_{e}$ shows a similar dependence for $\Phi_{e x t}<\Phi_{t h}$, but differs greatly when $\Phi_{e x t}>\Phi_{t h}$, where $j_{e} \propto \Phi_{e x t}$ is predicted. However, if electrons became the dominant means of charge conduction when the MIEC 
is reverse biased, then $\bar{t}_{i o n}$ will approach zero. This is not observed experimentally. In addition, ECRs, which operate in reverse bias, would be useless (or very inefficient) because the external power supply would drive electrons through the MIEC, at the expense of oxygen vacancies.

Figure 4.6.3c shows the dependence of $\bar{t}_{\text {ion }}$ on $\Phi_{\text {ext }}$ and further amplifies the inaccuracies incurred by the use of fixed boundary values. In line with our earlier discussion, it shows that, for potentialdependent boundary conditions, $\bar{t}_{i o n}$ approaches a minimum as $\Phi_{e x t} \rightarrow \Phi_{o c}$. As $\Phi_{e x t} \rightarrow \Phi_{o c}$, the external circuit grows increasingly resistive, forcing more electrons through the MIEC. Conversely, as the external circuit becomes less resistive (i.e., as $\Phi_{\text {ext }} \rightarrow \pm \infty$ ), fewer electrons flow through the MIEC and $\bar{t}_{i o n}$ approaches unity, since the fraction of current transported by electrons, in the MIEC, is decreasing. When fixed boundary values are used, no minimum is observed and $\bar{t}_{i o n}$ decreases monotonically in reverse bias, implying that electronic defects become the dominant means of charge conduction when the MIEC is reverse biased. That is not observed in practice, however, because the external circuit remains more electrically conductive than the MIEC.

Figure 4.6.3d is a plot of the current efficiency, $\zeta_{J}$, and power efficiency, $\zeta_{P}$, as a function of $\Phi_{\text {ext }}$ (for $0 \leq \Phi_{e x t} \leq \Phi_{o c}$ ). The plots show that $\zeta_{J}$ and $\zeta_{P}$ fall rapidly as $\Phi_{e x t} \rightarrow \Phi_{o c}$, due to the increase in $J_{e}$. These results are typical, however, it is observed that the use of fixed boundary values results in an overestimation of $\zeta_{J}$ and $\zeta_{P}$. Currently, extensive research is being conducted on acceptor-doped ceria because of its potential as an intermediate temperature $\left(500{ }^{\circ} \mathrm{C}\right.$ to $\left.700{ }^{\circ} \mathrm{C}\right) \mathrm{SOFC}$ electrolyte [133]. Much of this research focuses on finding better electrode materials for ceria-based electrolytes, because theoretical predictions suggest higher efficiencies are possible than are being obtained experimentally $[123,134]$. It is true that better electrodes are needed, but the targeted SOFC efficiencies may have been set too high because of inaccuracies incurred by the use of fixed boundary concentrations in the theoretical models.

\subsubsection{Non-Linear Potential Model}

Above, the impact of potential-dependent, versus fixed, boundary conditions on defect distribution and transport in oxide MIECs was emphasized. We now examine the effects of not assuming a linear potential (using only potential-dependent boundary conditions).

\section{Derivation}


To obtain an expression relating $c_{V}(x), \phi(x)$ and $x$ we take the gradient of Eq. (4.6.25), replacing $c_{e}$ with $c_{V}$ through Eq. (4.6.12), yielding:

$$
z_{V}\left(u_{e}-u_{V}\right) \nabla\left(c_{V} \nabla \phi\right)=u_{e} c_{A} \nabla^{2} \phi-z_{V}\left(D_{e}-D_{V}\right) \nabla^{2} c_{V}
$$

Differentiating, Eq. (4.6.1) and combining it with Eq. (4.6.32) yields for the steady state $\left(\nabla j_{i}=0\right)$

$$
\nabla^{2} \phi=\left(z_{V}-z_{e}\right)\left(z_{e} q c_{A}\right)^{-1} k_{B} T \nabla^{2} c_{V}=\lambda \nabla^{2} c_{V}
$$

where $\lambda=\left(z_{V}-z_{e}\right) k_{B} T /\left(z_{e} q c_{A}\right)$. Given the usual boundary conditions $\left(c_{i}=c_{i_{0}}\right.$ at $x=0$ and $c_{i}=c_{i_{L}}$ at $x=$ $L)$, Eq. (4.6.33) has solution:

$$
c_{V}(x)-c_{V_{0}}-\left(\phi(x)-\phi_{0}\right) \lambda^{-1}=-\left[\left(\phi_{L}-\phi_{0}\right) \lambda^{-1}-\left(c_{V_{L}}-c_{V_{0}}\right)\right] x / L=-\gamma x
$$

where $\gamma=\left(\phi_{L}-\phi_{0}\right) \lambda^{-1} L^{-1}-\left(c_{V_{L}}-c_{V_{0}}\right) L^{-1}$

Eqs. (4.6.34) provides an explicit relationship between $c_{V}(x), \phi(x)$ and $x$ which shows that $\nabla^{2} \phi \rightarrow$ 0 as $c_{A} \rightarrow \infty(\lambda \rightarrow 0)$ or $\nabla c_{V} \rightarrow 0$. In other words, a Laplacian potential distribution is applicable only in the absence of concentration gradients in the ionic species. Nevertheless, Eq. (4.6.34) is not useful, by itself, for obtaining defect concentration profiles and transport properties of the MIEC. Therefore, to obtain expressions for $c_{V}(x)$, Eqs. (4.6.1), (4.6.2) and (4.6.12) are combined to yield:

$$
\left(z_{V} u_{e} j_{V}-u_{V} j_{e}\right) c_{V}-j_{V} u_{e} c_{A}=q D_{e} D_{V} k_{B}^{-1} T^{-1}\left[z_{V}\left(z_{V}-z_{e}\right) c_{V}-c_{A}\right] \nabla c_{V}
$$

by eliminating $\nabla \phi$. Given the boundary conditions $\left(c_{i}=c_{i_{0}}\right.$ at $\left.x=0\right)$, Eq. (4.6.35) has solution

$$
c_{V}(x)-c_{V_{0}}+\frac{z_{V}^{2} u_{e} j_{V}+u_{V} j_{e}}{z_{V} u_{e} j_{V}-u_{V} j_{e}} \cdot \frac{c_{A}}{z_{V}\left(z_{V}-z_{e}\right)} \ln \frac{c_{V}(x)-\frac{j_{V} u_{e} c_{A}}{z_{V} u_{e} j_{V}-u_{V} j_{e}}}{c_{V_{0}}-\frac{j_{V} u_{e} c_{A}}{z_{V} u_{e} j_{V}-u_{V} j_{e}}}=k_{B} T \frac{z_{V} u_{e} j_{V}-u_{V} j_{e}}{z_{V}\left(z_{V}-z_{e}\right) q D_{e} D_{V}} x
$$

which, through comparison with Eq. (4.6.34) and boundary conditions $\left(c_{i}=c_{i_{L}}\right.$ at $\left.x=L\right)$ yields

$$
\begin{gathered}
c_{V}(x)-c_{V_{0}}-\frac{\left(D_{V} \gamma-j_{V}\right) c_{A}}{z_{V}\left(z_{V}-z_{e}\right) D_{V} \gamma} \cdot \ln \frac{z_{V}\left(z_{V}-z_{e}\right) D_{V} \gamma c_{V}(x)-j_{V} c_{A}}{z_{V}\left(z_{V}-z_{e}\right) D_{V} \gamma c_{V_{0}}-j_{V} c_{A}}=-\gamma x \\
\phi(x)=\phi_{0}-\frac{\left(D_{V} \gamma-j_{V}\right) k_{B} T}{z_{V} q D_{V} \gamma} \cdot \ln \frac{z_{V}\left(z_{V}-z_{e}\right) D_{V} \gamma c_{V}(x)-j_{V} c_{A}}{z_{V}\left(z_{V}-z_{e}\right) D_{V} \gamma c_{V_{0}}-j_{V} c_{A}}
\end{gathered}
$$




$$
\phi_{L}-\phi_{0}=\Delta \phi=-\frac{\left(D_{V} \gamma-j_{V}\right) k_{B} T}{z_{V} q D_{V} \gamma} \cdot \ln \frac{z_{V}\left(z_{V}-z_{e}\right) D_{V} \gamma c_{V_{L}}-j_{V} c_{A}}{z_{V}\left(z_{V}-z_{e}\right) D_{V} \gamma c_{V_{0}}-j_{V} c_{A}}
$$

and

$$
\gamma=\frac{\Delta \phi}{\lambda L}-\frac{c_{V_{L}}-c_{V_{0}}}{L}=-k_{B} T \frac{z_{V} u_{e} j_{V}-u_{V} j_{e}}{z_{V}\left(z_{V}-z_{e}\right) q D_{e} D_{V}}
$$

The form of Eqs. (4.6.37) and (4.6.38) are similar to those derived by Liu [123], unlike Liu's equations, however, the constants are readily quantifiable-i.e., $\lambda, \gamma, c_{V_{0}}$ and $c_{V_{\mathrm{L}}}$ all relate directly to the operating conditions imposed on the MIEC. In addition, as expected, Eqs. (4.6.37) - (4.6.39) reduce to corresponding expressions from the linear potential model when $c_{A}$ is large (i.e., for $\nabla c_{V} \approx 0$ ). From Eqs. (4.6.9) through (4.6.12) we have

$$
\frac{1}{\bar{t}_{\text {ion }}}=1+\frac{\bar{\sigma}_{e}}{{\overline{\sigma_{V}}}_{V}}=1+\frac{z_{e}^{2} q^{2} j_{e}}{z_{V}^{2} q^{2} j_{V}} \cdot \frac{k_{B} T \ln \left(c_{V_{L}} / c_{V_{0}}\right)+z_{V} q \Delta \phi}{k_{B} T \ln \left(c_{e_{L}} / c_{e_{0}}\right)+z_{e} q \Delta \phi}
$$

which may be rewritten as

$$
j_{e}=j_{V} \frac{z_{V}^{2}}{z_{e}^{2}}\left(\frac{1}{\bar{t}_{\text {ion }}}-1\right)\left(\frac{k_{B} T \ln \left(c_{e_{L}} / c_{e_{0}}\right)+z_{e} q \Delta \phi}{k_{B} T \ln \left(c_{V_{L}} / c_{V_{0}}\right)+z_{V} q \Delta \phi}\right)
$$

Therefore, combining Eqs. (4.6.39) through (4.6.42) yields

$$
\begin{gathered}
\gamma=-\frac{j_{V} k_{B} T}{\left(z_{V}-z_{e}\right) q D_{e} D_{V}}\left[u_{e}-u_{V} \frac{z_{V}}{z_{e}^{2}}\left(\frac{1}{\bar{t}_{\text {ion }}}-1\right)\left(\frac{k_{B} T \ln \left(c_{e_{L}} / c_{e_{0}}\right)+z_{e} q \Delta \phi}{k_{B} T \ln \left(c_{V_{L}} / c_{V_{0}}\right)+z_{V} q \Delta \phi}\right)\right] \\
j_{V}=-\frac{\left(z_{V}-z_{e}\right) q D_{e} D_{V} \gamma}{k_{B} T}\left[u_{e}-u_{V} \frac{z_{V}}{z_{e}^{2}}\left(\frac{1}{\bar{t}_{\text {ion }}}-1\right)\left(\frac{k_{B} T \ln \left(c_{e_{L}} / c_{e_{0}}\right)+z_{e} q \Delta \phi}{k_{B} T \ln \left(c_{V_{L}} / c_{V_{0}}\right)+z_{V} q \Delta \phi}\right)\right]^{-1}
\end{gathered}
$$

and

$$
\Delta \phi=-\left[\frac{k_{B} T}{z_{V} q}+\frac{\left(z_{V}-z_{e}\right) D_{e}}{z_{V}}\left[u_{e}-u_{V} \frac{z_{V}}{z_{e}^{2}}\left(\frac{1}{\bar{t}_{i o n}}-1\right)\left(\frac{\left.\ln \frac{c_{e_{L}}}{c_{e_{0}}}+\frac{z_{e} q}{k_{B} T} \Delta \phi\right)}{\left.\ln \frac{c_{V_{L}}}{c_{V_{0}}}+\frac{z_{V} q}{k_{B} T} \Delta \phi\right)}\right]\right]^{-1}\right] \cdot \ln \frac{c_{V_{L}}-\frac{j_{V} c_{A}}{z_{V}\left(z_{V}-z_{e}\right) D_{V} \gamma}}{c_{V_{0}}-\frac{j_{V} c_{A}}{z_{V}\left(z_{V}-z_{e}\right) D_{V} \gamma}}
$$

Finally by combining Eqs. (4.6.15), (4.6.21) and (4.6.45) we obtain 


$$
\begin{aligned}
& \Phi_{e x t}-\Phi_{t h}-\frac{k_{B} T}{z_{V} q} \ln \frac{c_{V_{L}}}{c_{V_{0}}} \\
& =-\left[\frac{k_{B} T}{z_{V} q}+\frac{\left(z_{V}-z_{e}\right) D_{e}}{z_{V}}\left[u_{e}-u_{V} \frac{z_{V}}{z_{e}^{2}}\left(\frac{\Phi_{t h}}{\eta+\Phi_{e x t}}-1\right)\left(\frac{\ln \frac{c_{e_{L}}}{c_{e_{0}}}-\frac{z_{e}}{z_{V}} \ln \frac{c_{V_{L}}}{c_{V_{0}}}+\frac{z_{e} q}{k_{B} T}\left(\Phi_{e x t}-\Phi_{t h}\right)}{\frac{z_{V} q}{k_{B} T}\left(\Phi_{e x t}-\Phi_{t h}\right)}\right)\right]^{-1}\right]
\end{aligned}
$$

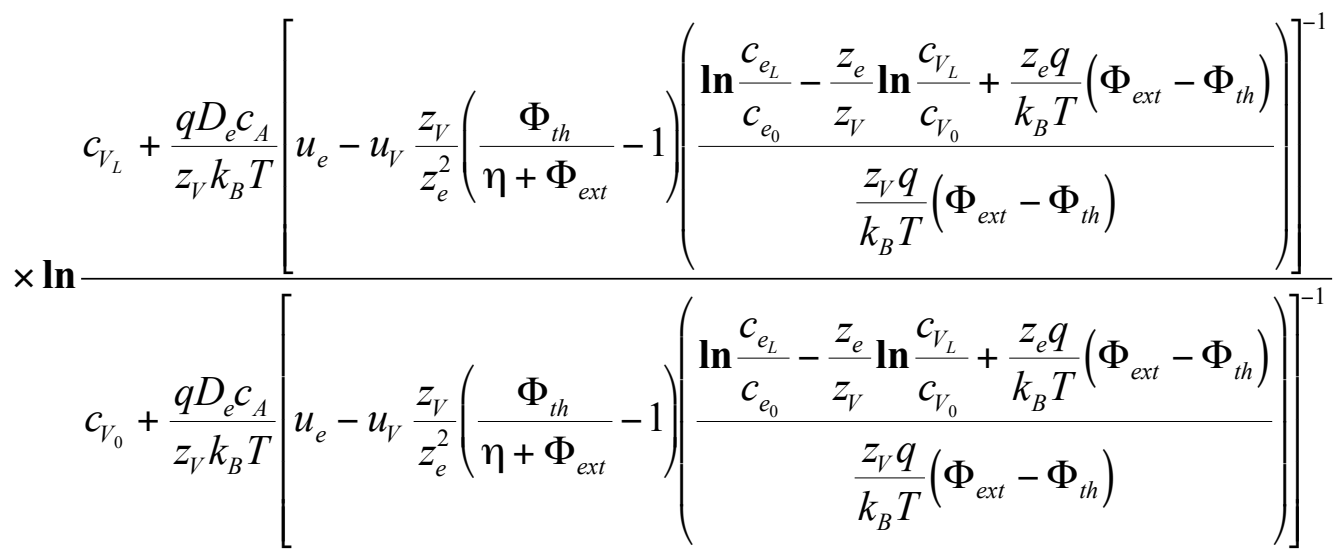

by which (along with the boundary values obtained from Eq. (4.6.22) through (4.6.24)) we may obtain unique values of $\eta$ for each input value of $\Phi_{\text {ext }}$ (i.e., for any given operating condition).

The transport properties, concentration and potential distribution for any MIEC may now be obtained through numerical solution of Eqs. (4.6.46) and the substitution of those results into Eqs. (4.6.37), (which must also be solved numerically) (4.6.38), (4.6.41), (4.6.42) and (4.6.44).

\section{Open-Circuit Voltage}

From thermodynamics, the open-circuit voltage, $\Phi_{\text {oc }}$, of an SOFC is defined as follows

$$
\Phi_{o c}=\frac{1}{2 z_{V} q} \int_{\mu_{\mathrm{O}_{2}, 0}}^{\mu_{\mathrm{O}_{2}, L}} t_{i o n} \cdot \mathrm{d} \mu_{\mathrm{O}_{2}}
$$

where $\mu_{\mathrm{O}_{2}, 0}$ and $\mu_{\mathrm{O}_{2}, 0}$ refer to the chemical potentials at $x=0$ and $x=L$, respectively, i.e., on either side of the SOFC. Substituting Eqs. (4.6.3), (4.6.4), (4.6.6), (4.6.12), (4.6.16) and (4.6.19) into Eq. (4.6.47) and integrating yields

$$
\Phi_{o c}=-\frac{k_{B} T}{z_{V} q}\left\{\frac{1-2 \Theta z_{V}}{1+\Theta z_{V}} \ln \frac{\left(1+\Theta z_{V}\right) c_{V_{L}}-\Theta c_{A}}{\left(1+\Theta z_{V}\right) c_{V_{0}}-\Theta c_{A}}+2 \ln \frac{z_{V} c_{V_{L}}-c_{A}}{z_{V} c_{V_{0}}-c_{A}}\right\}
$$


However, this result, Eq. (4.6.48), which is derived independently from the non-linear potential model, is identical to Eq. (4.6.39) in open-circuit conditions. This is easily verified by substituting the appropriate terms (e.g., using Eq. (4.6.16) (4.6.21), (4.6.40) and the Nernst equation: $2 z_{V} q \Phi_{\text {th }}=$ $\left.k_{B} T \ln \left(P_{\mathrm{O}_{2}, \mathrm{~L}} / P_{\mathrm{O}_{2}, 0}\right)\right)$ into either expression to obtain the other. This is significant because it thereby confirms the veracity of the non-linear potential model.

\section{Results and Discussion}

Figure 4.6.4 shows the spatial distribution of point defects in the MIEC, comparing the results of the Linear Potential Model and the Non-Linear Potential Model. Figure 4.6.4a shows that, in opencircuit conditions $\left(\Phi_{\text {ext }}=\Phi_{o c}\right)$, there is little difference between the predictions of either model. Moreover, although not shown in the figure, as $\Delta \phi \rightarrow 0\left(\Phi_{\text {ext }} \rightarrow \Phi_{t h}\right)$ the difference between both models disappears completely. This is directly attributable to the relation between $\phi$ and $c_{V}$ in Eqs. (4.6.33) and (4.6.34), which show that since $\nabla^{2} \phi \propto \nabla\left(\nabla c_{V}\right)$, then as $\nabla c_{V} \rightarrow 0$, $\phi$ becomes a linear function of $x$. The converse of this argument is demonstrated in Fig. 4.6.4b which shows a significant difference between both models in short circuit conditions $\left(\Phi_{\text {ext }}=0\right)$ where $\left|\nabla c_{V}\right|>>0$. Figure 4.6.4b also shows that, in short circuit conditions, the defect concentration gradients are not confined to the "near- boundary" areas. Instead, the concentration gradients spread across much of the MIEC. Overall, the results in Fig. 4.6.4 suggest that, the assumption of a linear electric field is more suitable for near open-circuit conditions.
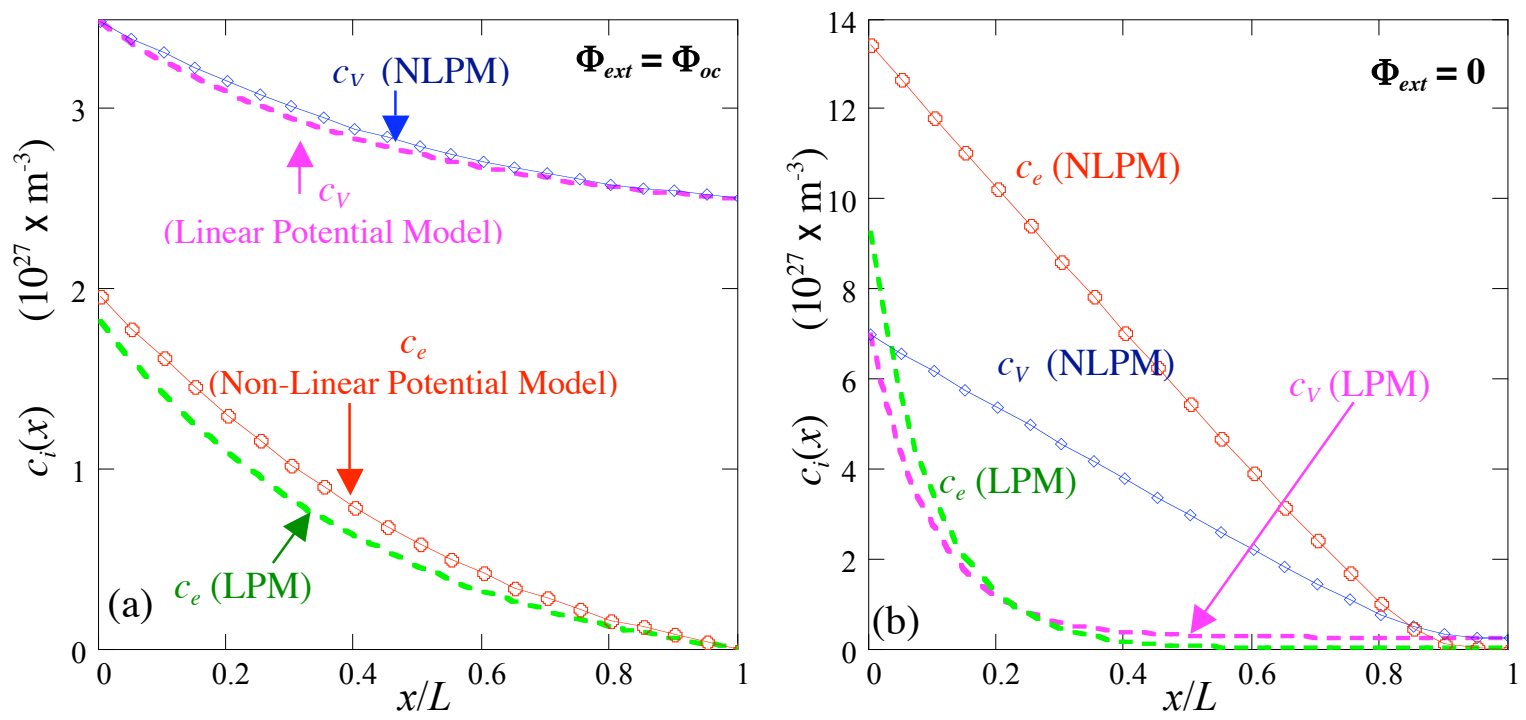

Figure 4.6.4. Comparison of concentration profiles from the linear potential model (LPM) and nonlinear potential model (NLPM) for (a) open-circuit $\left(\Phi_{\text {ext }}=\Phi_{o c}\right)$ and (b) short-circuit $\left(\Phi_{\text {ext }}=0\right)$ conditions. 


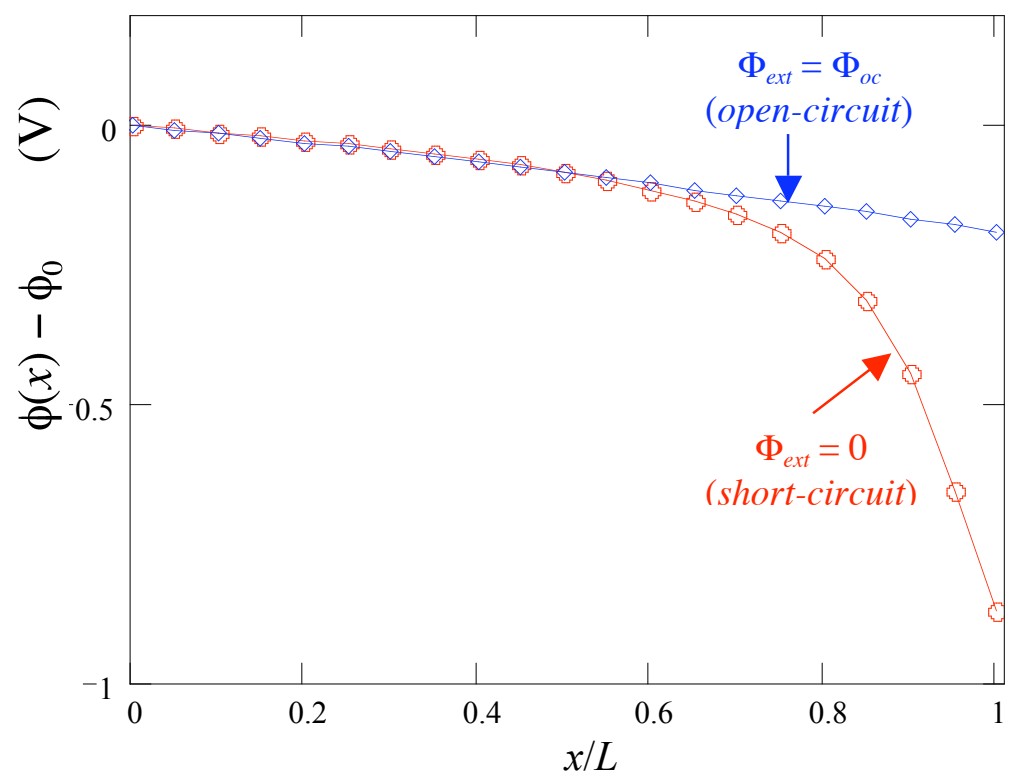

Figure 4.6.5. Spatial (Galvani) potential distribution in an (n-type) SDC electrolyte.

Figure 4.6.5 shows the dependence of the Galvani potential on $x$. Again, we see that, Eq. (4.6.24), the non-linearity of $\phi(x)$ increases as $\Phi_{e x t}$ moves away from $\Phi_{o c}$.

Many features of Fig. 4.6.6 discussed earlier for Fig. 4.6.3 are still applicable here and so are not reiterated. Instead, we highlight the differences between the results of the Linear Potential Model and the Non-Linear Potential Model. Firstly, in Fig. 4.6.6a, is the non-linear behavior of $j_{V}$ and $J$ (not shown) as $\Phi_{e x t} \rightarrow-\infty$, obtained from the Non-Linear Potential Model. In other words, by not forcing the potential to be linear, a non-Ohmic current-voltage response of the MIEC is observed. This behavior is observed experimentally in several MIEC systems [135]. Secondly, the Non-Linear Potential Model does not "force" $\nabla c_{V} \approx 0$ (see Eqs. (4.6.17) and (4.6.18), and related discussion, above), thereby (falsely) suppressing the electron concentration. Thus, in Figs. 4.6.6b, we observe a higher electronic flux for $-\Phi_{t h}<\Phi_{e x t}<\Phi_{t h}$. And higher electronic flux corresponds to lower $\bar{t}_{i o n}$, Fig. 4.6.6c, and reduced efficiency, Fig. 4.6.6d. Finally, in accordance with our earlier discussion for the two preceding figures, the results of both models coincide as $\Phi_{e x t} \rightarrow \Phi_{t h}$ in each case.

Figure 4.6.7 shows plots of the open-circuit voltage, $\Phi_{\mathrm{oc}}$, versus the $P_{\mathrm{O}_{2}}$ on the fuel side of an SDC concentration cell (Fig. 4.3.10) with air on the oxidizing side. The experimental data was collected at temperatures ranging from $500{ }^{\circ} \mathrm{C}$ to $750{ }^{\circ} \mathrm{C}$ and the voltage response of a zirconia oxygen sensor is included in the plots as a reference. The Non-Linear Potential model-specifically Eq. (4.6.39) or Eq. (4.6.48) — was fitted to the experimental data, through the usual least-squares fitting 

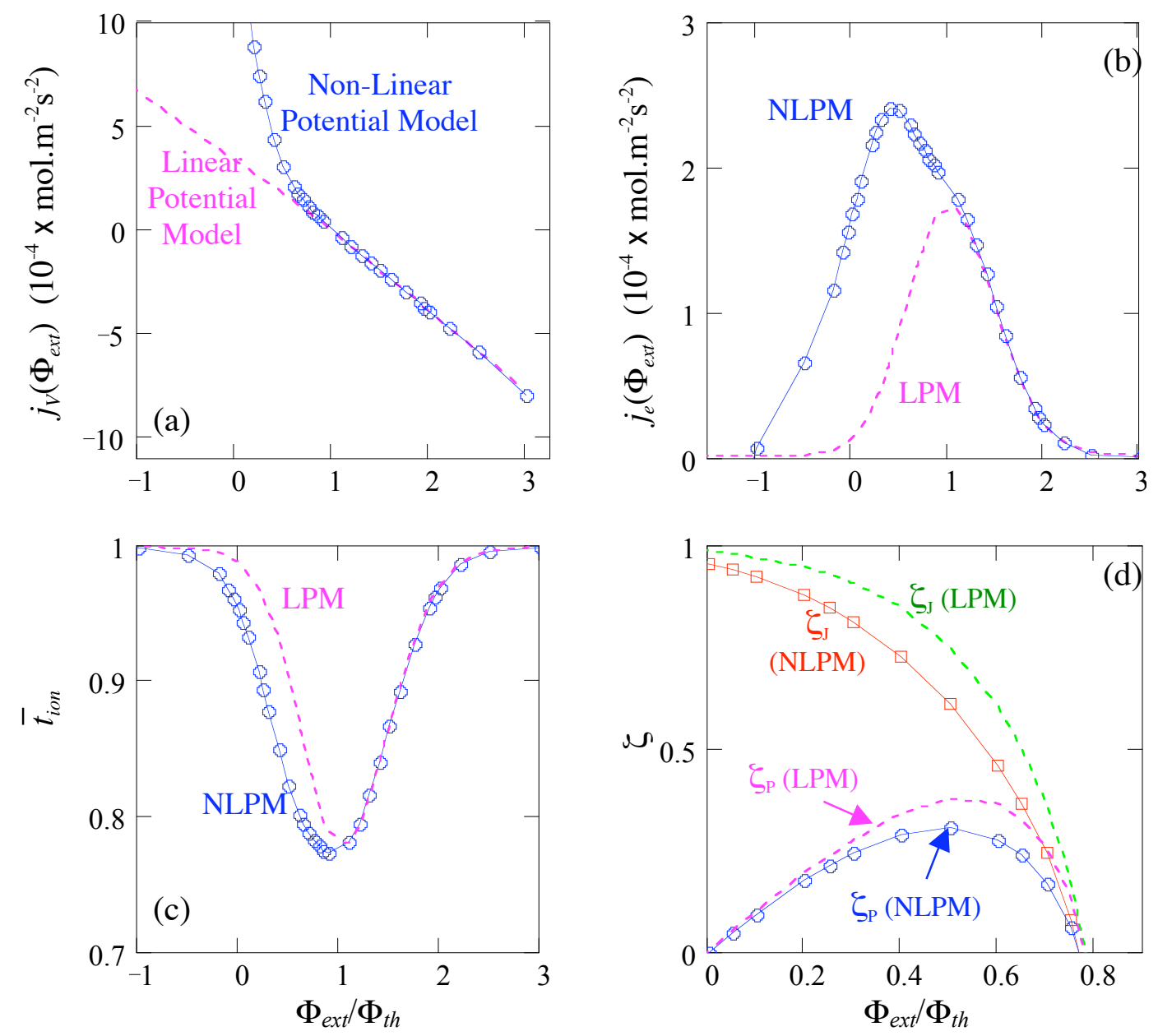

Figure 4.6.6. Comparing results of the Non-Linear Potential Model (NLPM) and the Linear Potential model (LPM) for (a) $j_{V}$, (b) $j_{e}$, (c) $\bar{t}_{i o n}$ and (d) $\zeta_{J}$ and $\zeta_{P}$, for $1 \mathrm{~mm}$ thick SDC electrolyte.

procedure, by adjusting $\Theta\left(D_{e} / 4 D_{V}\right)$ to maximize the resulting correlation coefficient. (The correlation coefficient was corrected for the fitting parameter, $\Theta$.) For $500{ }^{\circ} \mathrm{C}, 550{ }^{\circ} \mathrm{C}$ and $600{ }^{\circ} \mathrm{C}$ the square of the resulting correlation coefficients is 1.0 , which is indicative of an excellent fit. However, for $\mathrm{T} \geq$ $650{ }^{\circ} \mathrm{C}$, we experienced problems heating the sensor and so experimental errors in determining the $P_{\mathrm{O}_{2}}$ of the fuel increased. Nevertheless, the squares of the correlation coefficients obtained at these temperatures were $>0.95$, which is still very good.

Figure 4.6.7 also shows a decrease in $\Phi_{\mathrm{oc}}$ at the onset of electronic conduction in SDC, as expected, the same was not observed for the zirconia oxygen sensor. The plots also show that soon after the onset of electronic conduction, the $\Phi_{\mathrm{oc}}$ of the cell plateaus, i.e. becomes independent of $P_{\mathrm{O}_{2}}$. This phenomenon is readily explained by considering that since $2 c_{V_{0}} \approx c_{e_{0}}$ in highly reducing $P_{\mathrm{O}_{2}}$ (at $x$ $=0$, i.e., on the reducing side of the cell) when electronic conduction is significant, then for $2 \Theta>>1$ 

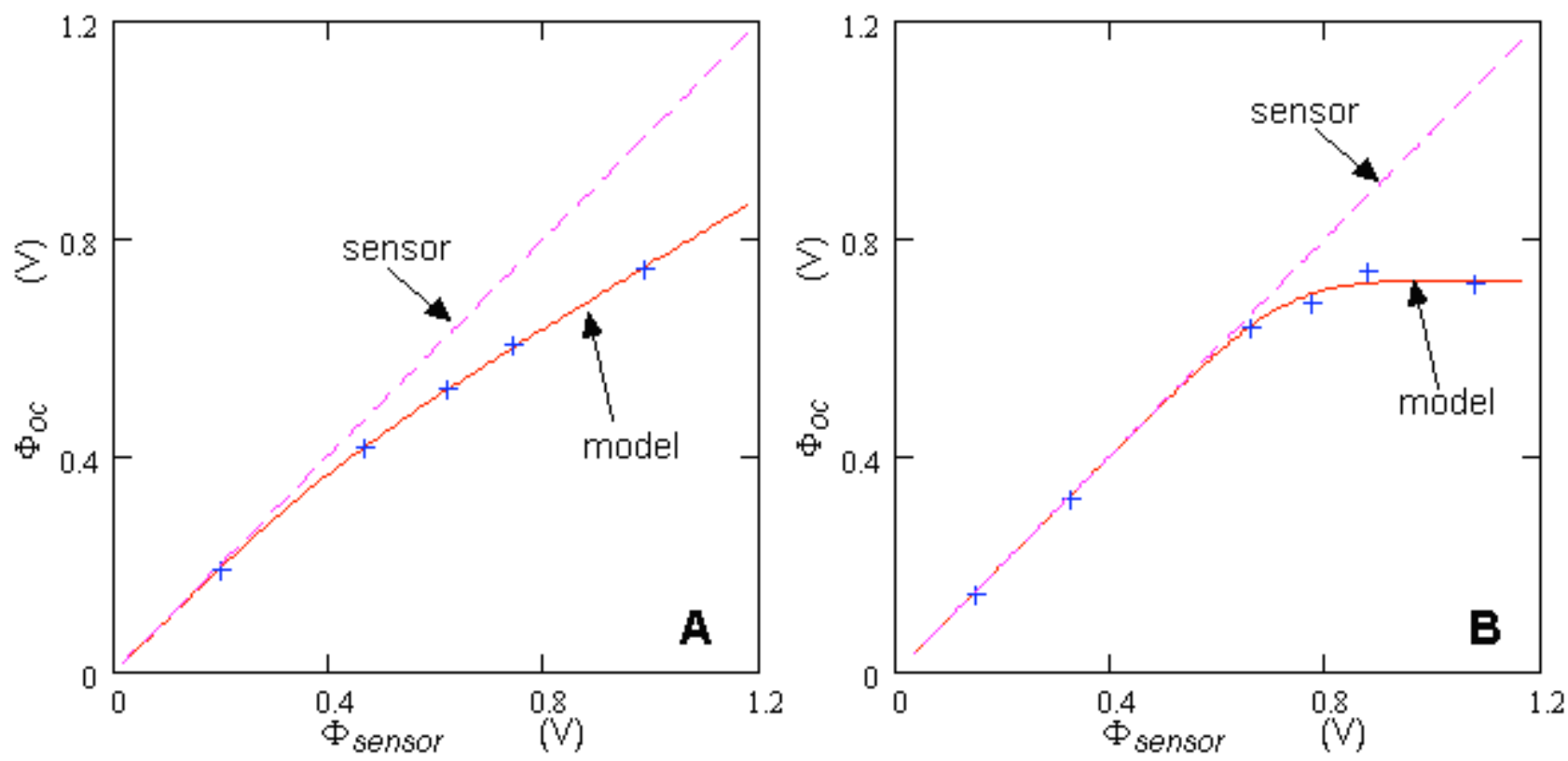

Figure 4.6.7. $\Phi_{\mathrm{oc}}$ vs. $P_{\mathrm{O}_{2}}$ on fuel side of SDC concentration cell (with air on the oxidizing side) for (a) $800{ }^{\circ} \mathrm{C}$ and (b) $500{ }^{\circ} \mathrm{C}$, showing the Non-Linear Potential Model (-) fitted to experimental data (•) and compared to the voltage response of a zirconia $\mathrm{O}_{2}$ sensor (---) used in the experiment.

(i.e., $D_{e} / D_{V}>>2$ ) Eq. (4.6.49)—combined with Eq. (4.6.12)—reduces to

$$
\Phi_{o c} \approx \frac{k_{B} T}{q} \ln \frac{c_{V_{L}}+\Theta c_{e_{L}}}{\Theta c_{e_{L}}}
$$

which is a constant since $c_{V_{L}}$ and $c_{e_{L}}$ are fixed by the $P_{\mathrm{O}_{2}}$ of air on the oxidizing side of the cell (i.e., $\left.P_{\mathrm{O}_{2} L}\right)$ which was fixed.

\subsubsection{Evaluating the Magnitude of the Equilibrium Constant, $\mathrm{K}_{\mathrm{r}}$}

In the equations developed in the preceding section, the equilibrium constant $K_{r}$ plays an important role in the performance of the MIEC. It is seen that the degree of mixed conduction increases with the magnitude of $K_{r}$. $K_{r}$, however, must be deduced from values obtained from experiment. In this section, four possible ways of evaluating $K_{r}$ are examined.

\section{Estimation of $K_{r}$ From Reduction of Ceria to Cerium (III) Oxide}

Some sense of the approximate value of $K_{r}$ may be deduced by considering the reduction of ceria to cerium (III) oxide, which may be written, using Kröger-Vink notation, as 


$$
2 \mathrm{Ce}_{\mathrm{Ce}}^{\times}+4 \mathrm{O}_{\mathrm{O}}^{\times} \leftrightarrow 2 \mathrm{Ce}_{\mathrm{Ce}}^{\times}+3 \mathrm{O}_{\mathrm{O}}^{\times}+\frac{1}{2} \mathrm{O}_{2} \quad K_{t}=c_{O}^{-1} P_{\mathrm{O}_{2}}^{\frac{1}{2}} / P_{\mathrm{O}_{2}, r e f}^{\frac{1}{2}}=K_{t}^{*} e^{-\frac{\Delta C_{t}}{k_{B} T}}
$$

where $K_{t}$ is the equilibrium constant and $\Delta G_{t}$ is the free energy for the reaction, $c_{O}$ is the concentration of oxygen atoms in oxygen sites, $K_{t}^{*}$ is a unit bearing pre-exponential and $P_{\mathrm{O}_{2} \text {,ref }}^{\frac{1}{2}}$ is the reference $P_{\mathrm{O}_{2}}$. However, as a first approximation, let Eq. (3.1) be rewritten as:

$$
2 \mathrm{Ce}_{\mathrm{Ce}}^{\times}+4 \mathrm{O}_{\mathrm{O}}^{\times} \leftrightarrow 2 \mathrm{Ce}_{\mathrm{Ce}}^{\prime}+3 \mathrm{O}_{\mathrm{O}}^{\times}+\mathrm{V}_{\mathrm{O}}^{\cdot \bullet}+\frac{1}{2} \mathrm{O}_{2} \quad \tilde{K}_{t}=\frac{c_{s p}^{2} c_{V} P^{\frac{1}{2}}}{c_{O} c_{M^{*}} P_{r e f}^{\frac{1}{2}}}=\tilde{K}_{t}^{*} e^{-\frac{\Delta \bar{\Delta}_{i t}}{\tilde{F}_{B}}}
$$

where $\tilde{K}_{t}$ is the equilibrium constant and $\Delta \tilde{G}_{t}$ is the Gibbs' free energy for the reaction and $\quad \tilde{K}_{t}^{*}$ is a unit bearing pre-exponential; $c_{s p}$ is the concentration of small polarons; and $c_{M}$ is the concentration of cations.

$\Delta \tilde{G}_{t}$ includes the enthalpy of vacancy formation as well as the entropy change in the ceria lattice hence it is unlikely that the reactions in Eqs. (4.6.50) and (4.6.51) are equivalent. Thus, one may write:

$$
\Delta \tilde{G}_{t}=\Delta G_{t}-k_{B} T \ln \theta \quad \tilde{K}_{t} \theta=K_{t}
$$

where $\theta(\theta>0)$ is a dimensionless correction term to account for the free energy difference between the transformation of $\mathrm{CeO}_{2}$ to $\mathrm{CeO}_{2-\delta}$ and $\mathrm{CeO}_{2}$ to $\mathrm{Ce}_{2} \mathrm{O}_{3}$. Hence, combining Eqs. (4.6.16), (4.6.51) and (4.6.52) (for $c_{e}<<c_{M}$ such that $c_{e}=c_{s p}$ and $c_{M^{*}} \approx c_{M}$ ) yields:

$$
K_{r} \theta=K_{t} c_{O} c_{M^{*}} P_{r e f}^{\frac{1}{2}}
$$

$K_{r} \theta$ may now be deduced from Eq. (4.6.53) by substituting $P_{r e f}=1 \mathrm{~atm} ., c_{M} \approx 2 \times 10^{28}$ atoms $/ \mathrm{m}^{3}, c_{A}=$ $0.5 \times 10^{28}$ atoms $/ \mathrm{m}^{3}$ - SDC lattice constant $=0.544 \mathrm{~nm}[133]-c_{O} \approx 4.75 \times 10^{28}$ atoms $/ \mathrm{m}^{3}$ and $\log \left(K_{t}\right)$ $=-11.595$ (obtained from thermodynamic tables) at $1073 \mathrm{~K}$, which yields

$$
K_{r} \theta=10^{73.7} \mathrm{~m}^{-9} \mathrm{~atm} \cdot{ }^{1 / 2}
$$

If $\theta$ can be quantified, or is close to unity, then this approach provides a good estimate of $K_{r}$. Without more rigorous thermodynamic analysis, it is difficult to ascertain the true magnitude and origin of $\theta$. Nevertheless, this calculation should give some idea of the magnitude of $K_{r}$.

\section{Estimation of $K_{r}$ from Thermogravimetry}

In principle, the best way to determine $K_{r}$ is through thermogravimetric experiments, which are designed to give the weight change of an MIEC sample (and hence the concentration of defects) as a 
function of $P_{\mathrm{O}_{2}}$. However, it is usually very difficult to get accurate and reproducible data from thermogravimetric experiments because of the high sensitivity required to detect very small changes in the mass of the specimen. Nevertheless, Kobayashi et al. [131] recently determined the oxygen/metal atom ratios, $c_{\mathrm{O}} /\left(c_{M}+c_{A}\right)$, for $\mathrm{SDC}$ for various temperatures and pressures. Oxygen vacancy concentration is related to the $c_{\mathrm{O}} /\left(c_{M}+c_{A}\right)$ ratio as follows:

$$
c_{V}=2\left(c_{M}+c_{A}\right)-c_{\mathrm{O}}=\left(c_{M}+c_{A}\right)\left[2-c_{\mathrm{O}} /\left(c_{M}+c_{A}\right)\right]
$$

Substituting Eq. (4.6.54) into Eq. (4.6.22) and rearranging it yields for $K_{r}$ :

$$
K_{r}^{\frac{1}{2}}=\frac{4}{3} P^{\frac{1}{4}} c_{V}^{\frac{3}{2}}-\frac{4}{3} P^{\frac{1}{4}}\left(\frac{1}{2} c_{A}\right)^{\frac{3}{2}}=\frac{4}{3} P^{\frac{1}{4}}\left\{\left(c_{M}+c_{A}\right)\left[2-c_{\mathrm{O}} /\left(c_{M}+c_{A}\right)\right]\right\}^{\frac{3}{2}}-\frac{4}{3} P^{\frac{1}{4}}\left(\frac{1}{2} c_{A}\right)^{\frac{3}{2}}
$$

$K_{r}$ is calculated from Eq. (3.6), for $c_{\mathrm{O}} /\left(c_{M}+c_{A}\right)$ ratios in the range 0.186 to 0.189 at $800{ }^{\circ} \mathrm{C}$ where $c_{M}=$ $2 \times 10^{28} \mathrm{~m}^{-3}-\mathrm{SDC}$ lattice constant $=0.544 \mathrm{~nm}[133]-$ and $c_{A}=5 \times 10^{27} \mathrm{~m}^{-3}$; the results are shown in Table 4.6-2. The values calculated for $K_{r}$ are narrowly distributed and so the standard deviation of the data set for $\ln K_{r}$ is $0.263^{4}\left[\ln \left(\mathrm{m}^{-9} \mathrm{~atm}^{1 / 2}\right)\right]$, which is $<0.2 \%$ in the worst case. The average value of $K_{r}$ in Table 4.6-2 is $9.97 \times 10^{71} \mathrm{~m}^{-9} \mathrm{~atm}^{1 / 2}$.

\section{Estimation of $K_{r}$ from Conductivity Measurements}

$K_{r}$ may also be determined from conductivity measurements. This method is attractive because in principle electrode effects (overpotentials, electrode resistance) can be separated from the bulk properties. Combining Eqs. (4.6.22) and (4.6.23) with the usual definitions of the total conductivity, $\sigma_{t o t}$, Eq. (4.6.5) and ionic transference number, $t_{i o n}$, Eq. (4.6.6), of an $n$-type MIEC yields:

$$
\sigma_{t o t}=\frac{z_{V}^{2} q^{2} D_{V}}{k_{B} T}\left(c_{V}+\Theta c_{e}\right)=\frac{z_{V}^{2} q^{2} D_{V}}{k_{B} T}\left\{\left[\frac{3}{4} K_{r}^{\frac{1}{2}} P^{-\frac{1}{4}}+\left(\frac{c_{A}}{2}\right)^{\frac{3}{2}}\right]^{\frac{2}{3}}+\Theta K_{r}^{\frac{1}{2}} P^{-\frac{1}{4}}\left[\frac{3}{4} K_{r}^{\frac{1}{2}} P^{-\frac{1}{4}}+\left(\frac{c_{A}}{2}\right)^{\frac{3}{2}}\right]^{-\frac{1}{3}}\right\}
$$

Table 4.6-2. Calculated values of $K_{r}$ for SDC from thermogravimetry data of Kobayashi et al. [131].

\begin{tabular}{cccc}
\hline$c_{O} /\left(c_{M}+c_{A}\right)$ & $\log P_{\mathrm{O}_{2}}[\log (\mathrm{atm})]$. & $K_{r}\left(\mathrm{~m}^{-9} \mathrm{~atm}^{1 / 2}\right)$ & $\ln K_{r}\left(\mathrm{~m}^{-9} \mathrm{~atm}^{1 / 2}\right)$ \\
\hline 1.86 & -20.5 & $6.732 \times 10^{71}$ & 165.390 \\
1.87 & -19.5 & $1.149 \times 10^{72}$ & 165.925 \\
1.88 & -18.75 & $1.159 \times 10^{72}$ & 165.934 \\
1.89 & -17.55 & $1.102 \times 10^{72}$ & 165.883 \\
\hline
\end{tabular}

\footnotetext{
4 The geometric mean and variance were required to calculate the standard deviation in the $K_{r}$ values.
} 
and

$$
t_{i o n}=\sigma_{V}\left(\sigma_{V}+\sigma_{e}\right)^{-1}=\left[1+\Theta K_{r}^{\frac{1}{2}} P^{-\frac{1}{4}}\left(\frac{3}{4} K_{r}^{\frac{1}{2}} P^{-\frac{1}{4}}+\frac{1}{2 \sqrt{2}} c_{A}^{\frac{3}{2}}\right)^{-1}\right]^{-1}
$$

respectively, where $q$ is the elementary electronic charge, $D$ is diffusivity and $\Theta$ is the ratio of electronic and ionic diffusivities as follows:

$$
\Theta=z_{e}^{2} D_{e} / z_{V}^{2} D_{V}
$$

In addition, if $\frac{3}{4} K_{r}^{\frac{1}{2}} P^{-\frac{1}{4}} \ll\left(\frac{1}{2} c_{A}\right)^{\frac{3}{2}}$ (i.e., the electrolytic region) then Eqs. (4.6.56) and (4.6.57) reduce to

$$
\sigma_{\text {tot }} \approx \frac{q^{2} z_{V}^{2} D_{V} c_{A}}{2 k_{B} T}\left[1+\Theta K_{r}^{\frac{1}{2}}\left(\frac{1}{2} c_{A}\right)^{-\frac{3}{2}} P^{-\frac{1}{4}}\right]=\frac{q^{2} z_{V}^{2} D_{V} c_{A}}{2 k_{B} T}\left\{1+\beta^{\frac{1}{2}} P^{-\frac{1}{4}}\right\}
$$

and

$$
t_{i o n} \approx\left[1+\Theta K_{r}^{\frac{1}{2}} P^{-\frac{1}{4}}\left(\frac{1}{2 \sqrt{2}} c_{A}^{\frac{3}{2}}\right)^{-1}\right]^{-1}=\left(1+\beta^{\frac{1}{2}} P^{-\frac{1}{4}}\right)^{-1}
$$

respectively, where

$$
\beta=K_{r} \Theta^{2}\left(\frac{1}{2} c_{A}\right)^{-3}
$$

Figure 4.6.8 shows a comparison of $\sigma$ versus $P_{\mathrm{O}_{2}}$ as predicted by Eq. (4.6.56) with experimental data from Eguchi et al. [130]. The value of $D_{V}$ used for the plot was taken from the data of Eguchi et al. [26]. $K_{r}$ and $D_{e}$ (i.e., $\Theta$ ) were then adjusted to get the best fit of Eq. (4.6.56). It was found that the best fit was achieved for $\beta=1.6 \times 10^{-7} \mathrm{~atm}^{1 / 2}$ but, as shown in Table 4.6-3, that value was obtainable with various combinations of $K_{r}$ and $D_{e}$. In other words, by fitting Eq. (4.6.56) to the conductivity data, a unique value of $\Theta$ ( or $D_{e}$ ) can be found for any $K_{r}$ or vice versa. It should also be noted that, as a review by Steele [137] shows, there is considerable spread in the values of $D_{e}$ reported for acceptordoped ceria in the literature. The transference number, as given by Eq. (4.6.57), is also plotted in Figure 4.6.8 as a function of pressure using the same values used to fit Eq. (3.7) to the $\sigma$ versus $P_{\mathrm{O}_{2}}$ data of Eguchi et al. [130]. 


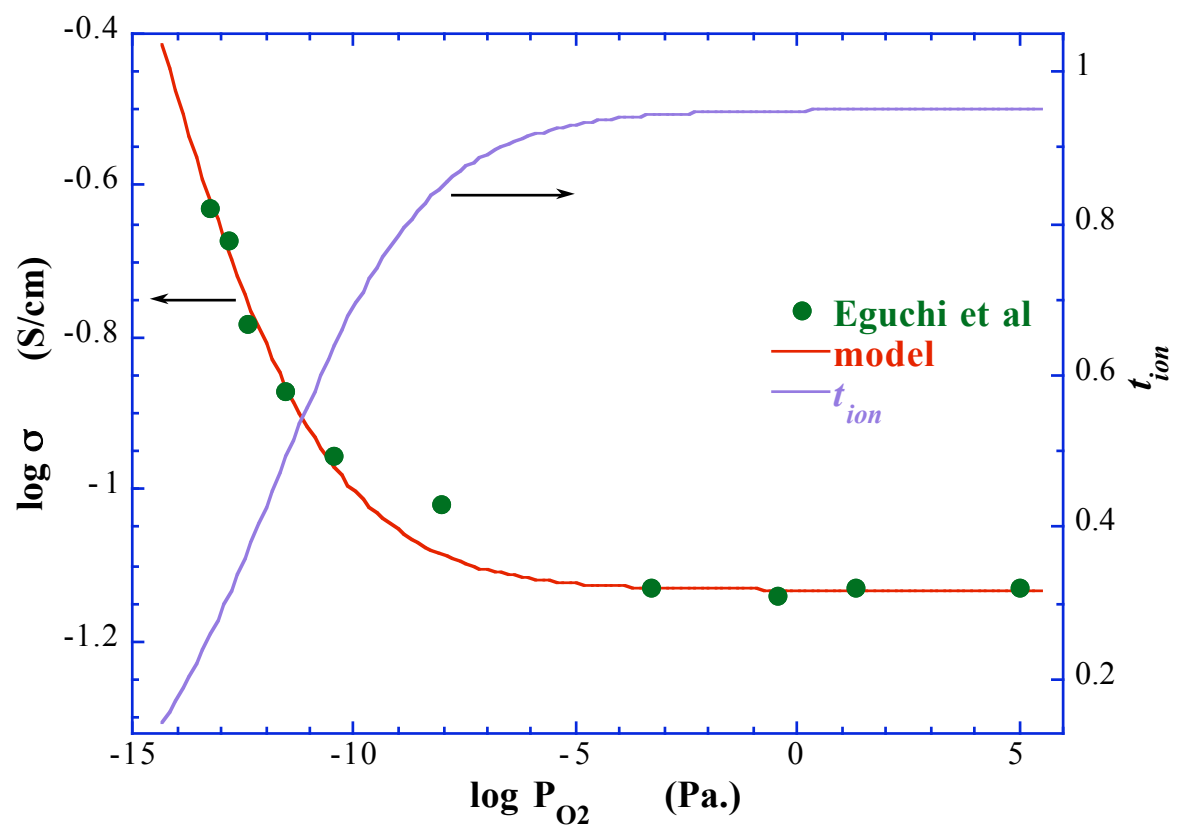

Figure 3. Modeled $\sigma\left(P_{\mathrm{O}_{2}}\right)$ fitted to experimental data from Eguchi et al. [26] for SDC.

Estimation of $K_{r}$ from open-circuit voltage

In this section, a method is developed for calculating $K_{r}$ from measurement of the open-circuit voltage, $\Phi_{o c}$, of a concentration cell with an MIEC membrane of thickness L. $\Phi_{o c}$ is defined as follows:

$$
\Phi_{o c}=\int_{\mu_{O_{2}}\left(P_{0}\right)}^{\mu_{O_{2}}\left(P_{L}\right)} \frac{t_{i o n}}{4 q} \cdot \mathrm{d} \mu_{\mathrm{O}_{2}}
$$

where $P_{0}$ and $P_{\mathrm{L}}$ are the oxygen partial pressures on either side of the cell (i.e., at $x=0$ and $x=\mathrm{L}$ respectively) and $\mu_{\mathrm{O}_{2}}$ is the chemical potential of oxygen. Substituting Eq. (4.6.57) into Eq. (4.6.62) and integrating yields:

Table 4.6-3. $\Theta, K_{r}$ and $\beta$ obtained by fitting Eq. (4.6.54) to SDC conductivity data of Eguchi et al. [130].

\begin{tabular}{cccc}
\hline$\Theta=D_{e} / 4 D_{V}$ & $K_{r}\left(\mathrm{~m}^{-9} \mathrm{~atm}^{1 / 2}\right)$ & $\ln K_{r}\left(\mathrm{~m}^{-9} \mathrm{~atm}^{1 / 2}\right)$ & $\beta\left(\mathrm{atm}^{1 / 2}\right)$ \\
\hline 0.1 & $1.0 \times 10^{77}$ & 177 & $8.0 \times 10^{-9}$ \\
1 & $1.3 \times 10^{76}$ & 175 & $1.0 \times 10^{-7}$ \\
10 & $2.0 \times 10^{74}$ & 171 & $1.6 \times 10^{-7}$ \\
100 & $2.0 \times 10^{72}$ & 166 & $1.6 \times 10^{-7}$ \\
140 & $1.0 \times 10^{72 \dagger}$ & 166 & $1.6 \times 10^{-7}$ \\
1000 & $2.0 \times 10^{70}$ & 162 & $1.6 \times 10^{-7}$ \\
\hline
\end{tabular}

$\dagger K_{r}$ calculated from thermogravimetric data of Kobayashi et al. [131]. 


$$
\Phi_{o c}=\frac{k T}{4 q} \ln \frac{P_{L}}{P_{0}}-\frac{k T}{q} \ln \frac{\left(\frac{3}{4}+\Theta\right) K_{r}^{\frac{1}{2}} P_{0}^{-\frac{1}{4}}+\left(\frac{1}{2} c_{A}\right)^{\frac{3}{2}}}{\left(\frac{3}{4}+\Theta\right) K_{r}^{\frac{1}{2}} P_{L}^{-\frac{1}{4}}+\left(\frac{1}{2} c_{A}\right)^{\frac{3}{2}}}=\Phi_{t h}-\frac{k T}{q} \ln \frac{\left(\frac{3}{4}+\Theta\right) K_{r}^{\frac{1}{2}} P_{0}^{-\frac{1}{4}}+\left(\frac{1}{2} c_{A}\right)^{\frac{3}{2}}}{\left(\frac{3}{4}+\Theta\right) K_{r}^{\frac{1}{2}} P_{L}^{-\frac{1}{4}}+\left(\frac{1}{2} c_{A}\right)^{\frac{3}{2}}}
$$

where $\Phi_{t h}$ is the theoretical (Nernst) potential. Eq. (4.6.63a) may be rearranged to give

$$
K_{r}^{\frac{1}{2}}=\frac{\left(\frac{1}{2} c_{A}\right)^{\frac{3}{2}}}{\left(\frac{3}{4}+\Theta\right)} \cdot \frac{1-\left(P_{L} / P_{0}\right)^{-\frac{1}{4}\left(1-\Phi_{o c} \mid \Phi_{t h}\right)}}{P_{0}^{-\frac{1}{4}}\left(P_{L} / P_{0}\right)^{-\frac{1}{4}\left(1-\Phi_{o c} \mid \Phi_{t h}\right)}-P_{L}^{-\frac{1}{4}}}
$$

Moreover for $\Theta>>3 / 4$, Eq. (4.6.63b) becomes

$$
\beta^{\frac{1}{2}} \approx \frac{1-\left(P_{L} / P_{0}\right)^{-\frac{1}{4}\left(1-\Phi_{o c} \Phi_{i l}\right)}}{P_{0}^{-\frac{1}{4}}\left(P_{L} / P_{0}\right)^{-\frac{1}{4}\left(1-\Phi_{o c} \mid \Phi_{l h}\right)}-P_{L}^{-\frac{1}{4}}}
$$

In principle, Eq. (4.6.63) allows $K_{r}$ to be determined from measurements of $\Theta$ and $\Phi_{o c}$ for a given $P_{0}$ and $P_{\mathrm{L}}$. However, $\Phi_{o c}$ may need to be corrected for electrode effects which alter true oxygen activity seen by the electrolyte at each interface. Furthermore, as Steele [137] shows, values for $D_{e}$ and hence $\Theta$, are difficult to obtain from experiment. And values available in the literature are inconsistent. Gödickemeier et al. $[150,151]$ modeled and evaluated the overpotentials for various electrodes on a SDC electrolyte. From their data for a $\mathrm{La}_{0.84} \mathrm{Sr}_{0.16} \mathrm{CoO}_{3}$ cathode and a $\mathrm{Ni}-\mathrm{Ce}_{0.9} \mathrm{Ca}_{0.1} \mathrm{O}_{1.9}$ cermet anode on an SDC electrolyte, values of $\Phi_{o c} \approx 800 \mathrm{mV}\left(\Phi_{o c} \approx 740 \mathrm{mV}\right.$ when corrected for electrode overpotentials $[150,151])$ and $\Phi_{t h} \approx 941 \mathrm{mV}$ for $P_{\mathrm{L}}=0.21 \mathrm{~atm}$. and $P_{0} \approx 4 \times 10^{-19} \mathrm{~atm}$. at $800{ }^{\circ} \mathrm{C}$ were obtained. $K_{r}$ is calculated for various values of $\Theta$, from Eq. (4.6.63a), using uncorrected and corrected (for electrode overpotential) data from Gödickemeier et al. [150,151] and the results are shown in Table 4.6-4.

As earlier, Eq. (4.6.63) shows that a unique value of $\Theta$ (or $D_{e}$ ) can be found for any $K_{r}$ or vice versa. Eq. (4.6.63) also shows that as $\Phi_{o c} / \Phi_{t h} \rightarrow 1$ (i.e., for good electrolytes) $\beta \rightarrow 0$. Conversely, as

Table 4.6-4. Calculated values of $\Theta$ and $K_{r}$ for SDC from $\Phi_{o c}$ data of Gödickemeier et al. [150, 151].

\begin{tabular}{cccccccc}
\hline$\Theta$ & $\begin{array}{c}K_{r} \\
\left(\mathrm{~m}^{-9} \mathrm{~atm} .{ }^{1 / 2}\right)\end{array}$ & $\ln K_{r}$ & $\beta \times 10^{-10}\left(\mathrm{~atm}^{1 / 2}\right)$ & & $\begin{array}{c}K_{r} \\
\left(\mathrm{~m}^{-9} \mathrm{~atm} .{ }^{1 / 2}\right)\end{array}$ & $\ln K_{r}$ & $\beta \times 10^{-10}\left(\mathrm{~atm}^{1 / 2}\right)$ \\
\cline { 2 - 3 } \cline { 6 - 7 } & uncorrected & & & & corrected \\
\hline 0.1 & $1.9 \times 10^{74}$ & 171 & 1.2 & & $2.9 \times 10^{73}$ & 169 & 0.19 \\
1 & $4.4 \times 10^{73}$ & 170 & 28 & & $6.8 \times 10^{72}$ & 168 & 4.4 \\
10 & $1.2 \times 10^{72}$ & 166 & 77 & & $1.8 \times 10^{71}$ & 164 & 12 \\
100 & $1.3 \times 10^{70}$ & 161 & 83 & & $2.1 \times 10^{69}$ & 160 & 13 \\
1000 & $1.3 \times 10^{68}$ & 157 & 83 & & $2.1 \times 10^{67}$ & 155 & 13 \\
\hline
\end{tabular}


$\Phi_{o c} / \Phi_{t h} \rightarrow 0$ (i.e., for poor electrolytes) $\beta \rightarrow \infty$. That is, the suitability of an $n$-type oxide MIEC as an electrolyte can be determined from a knowledge of $\beta$ which combines information on the reducibility of the MIEC, the relative diffusivity of the electronic and ionic defects, and the doping level.

\section{Summary}

There is good agreement between all of the experimentally based strategies used to calculate/estimate the magnitude of $K_{r}$ (especially for $\sim 1<\Theta<\sim 10$ ). Specifically, the results obtained from each method (using $\Theta=10)$ are $<5 \%$ apart for $\ln K_{r}\left(\propto \Delta G_{r}\right)$, which is well within experimental error. Such unanimity alludes to the correctness of the approaches and, therefore, the model-i.e., Eqs. (2.14). Admittedly, however, no error analysis was reported for any of the experimental data. In their absence confidence in the actual data, and any results educed from it, diminishes. Nevertheless, obtaining such similar results from such disparate sources and methods is, at the very least, reassuring. $K_{r}$ is a critical constant and it is worth the effort to find reliable ways of determining its value accurately.

In comparison to Table 4.6-4, Table 4.6-3 shows higher $K_{r}$ values for each $\Theta$ (or higher $\Theta$ for a given $K_{r}$ ) and, accordingly, higher $\beta$ values. The values of $\Theta$ and $K_{r}$ obtained from conductivity measurements (Table 4.6-3) may be considered to be "more correct" than those from $\Phi_{\text {oc }}$ measurements (Table 4.6-4) because of the absence of electrode effects and since conductivity measurements are made at (true versus local) equilibrium. Additionally, the lower $\beta$ values in Table 4.6-4 may be due to the calculations being made for a single $\left(P_{\mathrm{L}}, P_{0}, T\right)$ coordinate rather than a range, as is the case for the conductivity data, in Table 4.6-3. Hence, in principle, statistical errors are minimized for the latter.

Eqs. (4.6.56) and (4.6.63) show that it is difficult to separate $K_{r}$ and $D_{e}$ in experiments which measure properties that are dependent on both defect concentration $\left(K_{r}\right.$ and $\left.c_{A}\right)$ and diffusivity $(\Theta$ or $D_{e}$ ) - e.g., $\Phi_{o c}, \sigma$ or $t_{i o n}$ measurements. This may explain, somewhat, the variability found in the literature [137] for $D_{e}$ and in general suggests that caution should be applied whenever such experiments are used to determine these values. Moreover, alternative experiments, such as thermogravimetry (if acceptable repeatability and accuracy can be achieved), may prove to be more reliable in obtaining these constants.

Finally, by comparing the formalism used for the thermodynamic estimation of $K_{r}$, Eq. (4.6.53), with that used in the estimation from $\Phi_{o c}$ measurements, Eq. (4.6.63), one may surmise that $\theta$ is related 
to $\Theta$. Through (at this point, tenuous) extrapolation, one may further deduce that as $\theta \rightarrow 1$, Eq. (4.6.50) becomes more favorable than Eq. (4.6.51), i.e., cerium (IV) oxide will decompose to cerium (III) oxide rather than become non-stoichiometric. Conversely, as $\theta \rightarrow 0$, Eq. (4.6.50) is less favorable and departures from stoichiometry (within thermodynamic limits) are sustainable in cerium (IV) oxide.

\subsubsection{Conclusion}

Previous models for the transport properties and performance of MIECs in SOFC applications [121 - 124] excluded the effects of (a) the external potential (on the boundary values of the defect concentrations) and/or (b) a non-linear potential. We have included these effects in this work. Significantly, it was shown that excluding the effect of $\Phi_{\text {ext }}$ on the boundary conditions leads to results that contradict those obtained from experiment. Moreover, the exclusion leads to an overestimation of SOFC current and power efficiencies. We were further able to show that imposing a linear potential

distribution forces the MIEC to behave ohmically, for $\left|\Phi_{\text {ext }}\right|>0$, and causes overestimation of SOFC current and power efficiencies.

Beyond the pursuit of scientific knowledge, the importance of these results is their direct bearing on the engineering of SOFCs. Especially, re the determination of attainable SOFC efficiencies and the characterization of MIECs for said applications.

Finally, experimental and theoretical paths were explored for the determination of a crucial material property, the equilibrium constant, $K_{r}$. Significantly, good consistency was found between all the approaches evaluated. 


\subsection{Defect Transport in Bilayer Electrolytes}

In this section, a model for the performance and stability of cerialbismuth oxide bilayer electrolytes is developed. The purpose of this model is to determine the optimum relative thickness of the constituent layers of the bilayer electrolyte. This is an extension of earlier work, presented in section 4.6, on the transport properties of mixed ionic-electronic conductors by solution of the NernstPlanck equation with potential-dependent boundary conditions and without assuming a linear galvanic potential [136]. The results of the model demonstrate the feasibility and potential performance of the bilayer electrolyte.

\subsubsection{Bilayer Model}

We now apply earlier models (section 4.6) for defect transport in an SOFC electrolyte [136] to obtain the relationship between the thickness ratio of the constituent layers of the SDCIESB bilayer and the potential drop across either layer. An equivalent circuit of the bilayer electrolyte is shown in Fig. 4.7.1, which also comprises the equivalent circuit for a single layer for comparison.

\section{Derivation}

For the bilayer electrolyte, the ionic and electronic currents are the same in both layers, in steady state conditions. Therefore, for a bilayer consisting of an $\varepsilon$ layer (SDC), of thickness $L^{\varepsilon}$, and a $\delta$ layer (ESB), of thickness $L^{\delta}$, we have:

$$
J_{V}^{\varepsilon}=J_{V}^{\delta} \quad \text { or } \quad J_{e}^{\varepsilon}+J_{h}^{\varepsilon}=J_{e}^{\delta}+J_{h}^{\delta} \quad \text { or } \quad J^{\varepsilon}=J^{\delta}
$$

where $J_{V}, J_{e}, J_{h}$ and $J$ are the oxygen vacancy, electron, hole and total currents, respectively. So, to obtain an expression for the thickness ratio, $\tau=L^{\varepsilon} / L^{\delta}$, let us consider the following relationships for defect distribution and transport in a single-layer mixed ionic-electronic conductor (MIEC), which was derived in our earlier work [136] and presented in section 4.6:

$$
\Delta \phi=\phi_{L}-\phi_{0}=-\frac{D_{e, h} J_{V}+D_{V} J_{e, h}}{z_{e, h} D_{e, h} J_{V}+z_{V} D_{V} J_{e, h}} \cdot \frac{k_{B} T}{q} \ln \frac{z_{V} c_{V_{L}}-\frac{z_{e, h} D_{e, h} c_{A} J_{V}}{z_{e, h} D_{e, h} J_{V}+z_{V} D_{V} J_{e, h}}}{z_{V} c_{V_{0}}-\frac{z_{e, h} D_{e, h} c_{A} J_{V}}{z_{e, h} D_{e, h} J_{V}+z_{V} D_{V} J_{e, h}}}
$$

and 


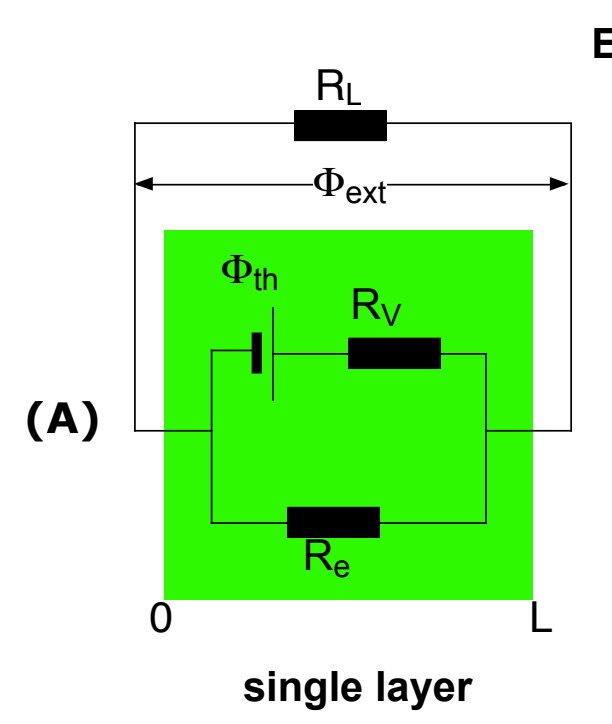

\section{Equivalent Circuits}

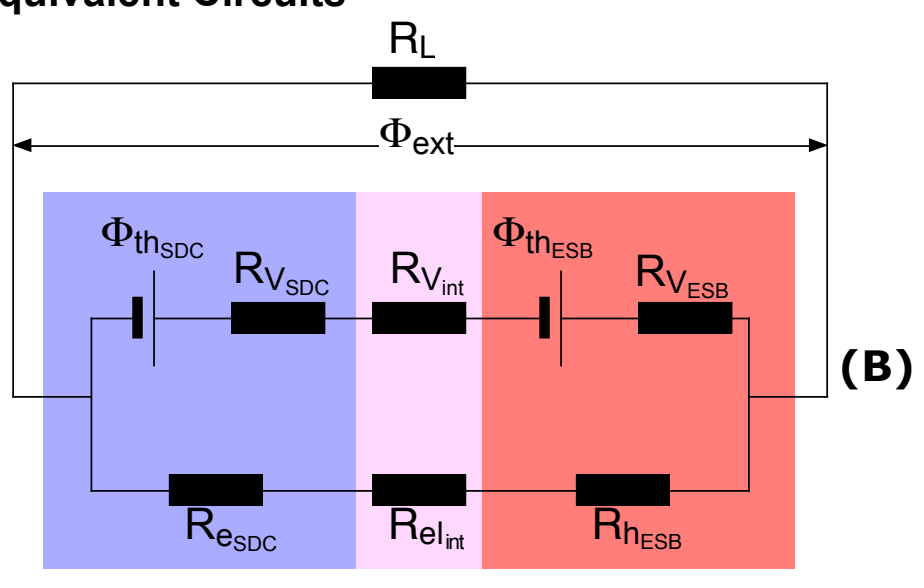

bilayer

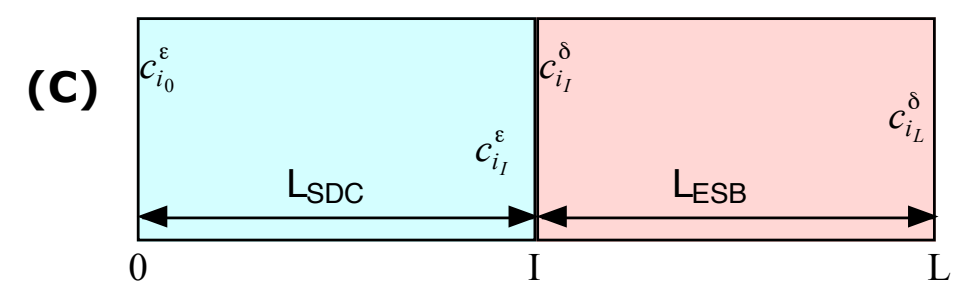

Figure 4.7.1. (a) Equivalent circuit for a single layer electrolyte, (b) equivalent circuit for a bilayer electrolyte and (c) schematic showing the boundary concentration values relative to the thickness of the bilayer and the thicknesses of the constituent layers.

$$
c_{V_{L}}-c_{V_{0}}+\frac{\frac{z_{e, h}}{z_{V}-z_{e, h}}\left(D_{e, h}+D_{V} \frac{J_{e, h}}{J_{V}}\right) c_{A}}{z_{e, h} D_{e, h}+z_{V} D_{V} \frac{J_{e, h}}{J_{V}}} \ln \frac{z_{V} c_{V_{L}}-\frac{z_{e, h} D_{e, h} c_{A} J_{V}}{z_{e, h} D_{e, h} J_{V}+z_{V} D_{V} J_{e, h}}}{z_{V} c_{V_{0}}-\frac{z_{e, h} D_{e, h} c_{A} J_{V}}{z_{e, h} D_{e, h} J_{V}+z_{V} D_{V} J_{e, h}}}=\frac{\frac{z_{e, h} J_{V}}{D_{V}}+\frac{z_{V} J_{e, h}}{D_{e, h}}}{z_{V}\left(z_{V}-z_{e, h}\right) q} L
$$

where $\phi$ is the Galvani potential, $D$ is diffusivity, $z$ is charge number, $q$ is elementary electron charge, $c$ is concentration, $k_{B}$ is Boltzmann's constant, $T$ is temperature and $L$ is electrolyte thickness. The subscripts 0 and $L$ refer to (boundary) conditions on either side of an electrolyte; $V$, $e$, and $h$ refer to vacancies, electron and holes respectively; $e, h$ refers to electrons or holes. Also, $c_{A}$ is the concentration of a trivalent acceptor-dopant. In ESB all the cations are trivalent and function as "acceptor-dopants on the fluorite lattice" [138]. That is, cubic bismuth oxide may be conceived as having a fluorite lattice in which the tetravalent host cation sites are filled with trivalent bismuth cations.

The ionic and electronic currents are constant in steady state and we define, the electronic-ionic current ratio, $r=-J_{e, h} / J_{V}$. Therefore, Eqs. (4.7.2) reduces (for either layer) to: 


$$
\Delta \phi=\phi_{L}-\phi_{0}=-\frac{D_{e, h}-D_{V} r}{z_{e, h} D_{e, h}-z_{V} D_{V} r} \cdot \frac{k_{B} T}{q} \ln \frac{z_{V}\left(z_{e, h} D_{e, h}-z_{V} D_{V} r\right) c_{V_{L}}-z_{e, h} D_{e, h} c_{A}}{z_{V}\left(z_{e, h} D_{e, h}-z_{V} D_{V} r\right) c_{V_{0}}-z_{e, h} D_{e, h} c_{A}}
$$

Since Eq. (4.7.3) is applicable to any single layer of the bilayer, we combine Eqs. (4.7.1) and (4.7.3) with the result (in typical atmospheres, SDC is $n$-type and ESB is $p$-type),

$$
J_{V}=\frac{z_{V}\left(z_{V}-z_{e, h}\right) q D_{e, h} D_{V}}{\left(z_{e, h} D_{e, h}-z_{V} D_{V} r\right) L}\left\{c_{V_{L}}-c_{V_{0}}+\frac{\frac{z_{e, h}}{z_{V}-z_{e, h}}\left(D_{e, h}-D_{V} r\right) c_{A}}{z_{e, h} D_{e, h}-z_{V} D_{V} r} \ln \frac{z_{V} c_{V_{L}}-\frac{z_{e, h} D_{e, h} c_{A}}{z_{e, h} D_{e, h}-z_{V} D_{V} r}}{z_{V} c_{V_{0}}-\frac{z_{e, h} D_{e, h} c_{A}}{z_{e, h} D_{e, h}-z_{V} D_{V} r}}\right\}
$$

and

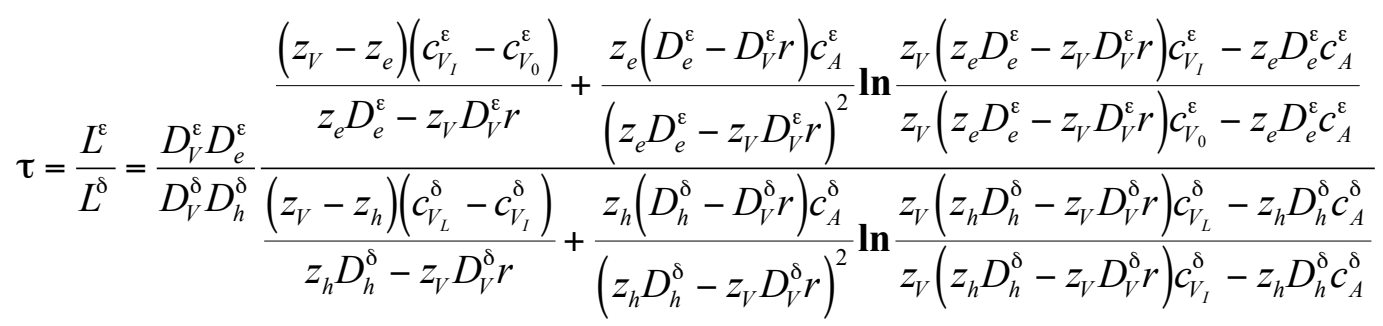

where the subscript " $\Gamma$ " refers to the interface, see Fig. 4.7.1.

Potential Differences. The Galvani potential drop across the bilayer, $\Delta \phi^{\varepsilon+\delta}$, may be obtained from the local equilibrium approximation $[136,139]$, as shown in Eqs. (4.6.16) through (4.6.21)

$$
\Delta \phi^{\varepsilon+\delta}=\Delta \phi^{\varepsilon}+\Delta \phi^{\delta}=\Phi_{e x t}-\left(\Phi_{t h}^{\varepsilon}+\Phi_{t h}^{\delta}-\Delta \phi_{i n t}\right)-\frac{k_{B} T}{z_{V} q} \ln \frac{c_{V_{L}}^{\delta}}{c_{V_{0}}^{\varepsilon}}=\Phi_{e x t}-\Phi_{t h}-\frac{k_{B} T}{z_{V} q} \ln \frac{c_{V_{L}}^{\delta}}{c_{V_{0}}^{\varepsilon}}
$$

where $\Phi_{\text {ext }}$ is the external voltage (i.e., the voltage drop across a load), $\Delta \phi_{\text {int }}$ is the voltage drop across the interface between the layers of the bilayer, and $\Phi_{t h}$ is the theoretical (Nernst) voltage. By considering the steady state flux across the interface between the constituent layers in the absence of external electrochemical gradients, it may be shown that:

$$
\Delta \phi_{\text {int }}=z_{V}^{-1} q^{-1} k_{B} T \ln \left(c_{V_{I}}^{\delta} / c_{V_{I}}^{\varepsilon}\right)
$$

Finally, to acquire expressions for the oxygen vacancy concentrations of the constituent layers at their interface, Eqs. (4.7.7) and (4.7.8) are substituted into Eq. (4.7.5) with the result:

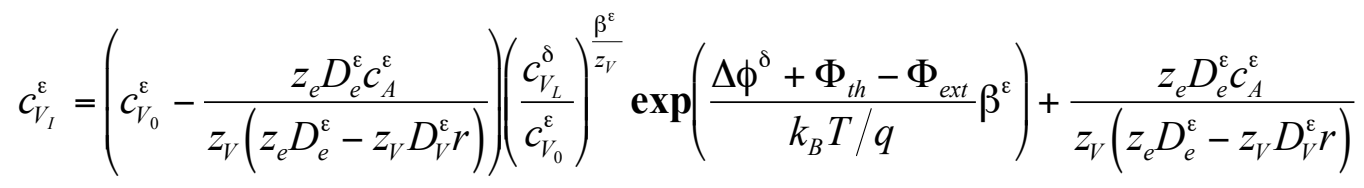


and

$$
c_{V_{I}}^{\delta}=\left(c_{V_{L}}^{\delta}-\frac{z_{h} D_{h}^{\delta} c_{A}^{\delta}}{z_{V}\left(z_{h} D_{h}^{\delta}-z_{V} D_{V}^{\delta} r\right)}\right) \exp \left(\frac{q}{k_{B} T} \cdot \frac{z_{h} D_{h}^{\delta}-z_{V} D_{V}^{\delta} r}{D_{h}^{\delta}-D_{V}^{\delta} r} \cdot \Delta \phi^{\delta}\right)+\frac{z_{h} D_{h}^{\delta} c_{A}^{\delta}}{z_{V}\left(z_{h} D_{h}^{\delta}-z_{V} D_{V}^{\delta} r\right)}
$$

where $\beta^{\varepsilon}=\left(z_{e} D_{e}^{\varepsilon}-z_{V} D_{V}^{\varepsilon} r\right) /\left(D_{e}^{\varepsilon}-D_{V}^{\varepsilon} r\right)$. Eqs. (4.7.9) and/or (4.7.10) may be combined with Eqs. (4.7.5) and (4.7.6) to obtain $J$ and $\tau$ as a function of $\Delta \phi^{\delta}$ and $r$.

Electronic-Ionic Current Ratio. In open-circuit conditions $r=1$, however, to quantify the functionality of $r$ in closed-circuit conditions, we need to derive an expression for $r$ in terms of the properties of the bilayer and said operating conditions. From Eqs. (4.6.15), (4.6.18) through (4.6.20) and (4.6.41) we have [136, 139]:

$$
r=\left(\frac{\Phi_{t h}}{\eta+\Phi_{e x t}}-1\right)\left(\frac{\Phi_{e x t}}{\Phi_{t h}-\Phi_{e x t}}\right) \quad \text { or } \quad \eta=\frac{\Phi_{t h}-\Phi_{e x t}}{r \Phi_{t h}+(1-r) \Phi_{e x t}}(1-r) \Phi_{e x t}
$$

\section{Boundary Conditions}

For MIECs, the defect concentration boundary values are set by the defect equilibria. From our previous work, we obtained relations for the functional dependence of $c_{V}$ and $c_{e}$ on $P_{\mathrm{O}_{2}}$ [140]. These are given below for $n$-type SDC, Eqs. (4.7.12) and (4.7.13), and $p$-type ESB, Eqs. (4.7.14) and (4.7.15), respectively,:

$$
\begin{gathered}
c_{V}\left(P_{\mathrm{O}_{2}}\right)=\left[\frac{3}{4} K_{r}^{\frac{1}{2}} P_{\mathrm{O}_{2}}^{-\frac{1}{4}}+\left(\frac{1}{2} c_{A}\right)^{\frac{3}{2}}\right]^{\frac{2}{3}} \\
c_{e}\left(P_{\mathrm{O}_{2}}\right)=K_{r}^{\frac{1}{2}} P_{\mathrm{O}_{2}}^{-\frac{1}{4}}\left[\frac{3}{4} K_{r}^{\frac{1}{2}} P_{\mathrm{O}_{2}}^{-\frac{1}{4}}+\left(\frac{1}{2} c_{A}\right)^{\frac{3}{2}}\right]^{-\frac{1}{3}} \\
c_{V}\left(P_{\mathrm{O}_{2}}\right)=\frac{1}{16} K_{m}\left(\sqrt{8 K_{m}^{-1} c_{A}+P_{\mathrm{O}_{2}}^{\frac{1}{2}}}-P_{\mathrm{O}_{2}}^{\frac{1}{4}}\right)^{2} \\
c_{h}\left(P_{\mathrm{O}_{2}}\right)=\frac{1}{4} K_{m} P_{\mathrm{O}_{2}}^{\frac{1}{4}}\left(\sqrt{8 K_{m}^{-1} c_{A}+P_{\mathrm{O}_{2}}^{\frac{1}{2}}}-P_{\mathrm{O}_{2}}^{\frac{1}{4}}\right)
\end{gathered}
$$


where $K_{r}$ and $K_{m}$ are equilibrium constants for the oxygen exchange between the oxide and gaseous $\mathrm{O}_{2}$ when written as follows:

$$
\begin{array}{lr}
\mathrm{O}_{\mathrm{O}}^{\times} \Leftrightarrow \mathrm{V}_{\mathrm{O}}^{\bullet \bullet}+2 e^{\prime}+\frac{1}{2} \mathrm{O}_{2} & K_{r}=c_{V} c_{e}^{2} P_{\mathrm{O}_{2}}^{\frac{1}{2}} \\
\mathrm{~V}_{\mathrm{O}}^{\bullet \bullet}+\frac{1}{2} \mathrm{O}_{2} \Leftrightarrow \mathrm{O}_{\mathrm{O}}^{\times}+2 h & K_{m}=c_{V}^{-1} c_{h}^{2} P_{\mathrm{O}_{2}}^{-\frac{1}{2}}
\end{array}
$$

Thus $K_{m}=\left(K_{i}\right)^{2} / K_{r}\left(K_{r}\right.$ is defined before and $K_{i}$ is the equilibrium constant for thermal generation of electron-hole pairs). The boundary values, $c_{i_{0}}$ (at $x=0$, at the anode) and $c_{i_{L}}$ (at $x=\mathrm{L}$, at the cathode) are had by appropriately substituting $P_{\mathrm{O}_{2}, 0}$ and $P_{\mathrm{O}_{2}, \mathrm{~L}}$ into Eqs. (4.7.13) through (4.7.16).

The total free energy of an electrochemical reaction has a chemical and an electrical part [141, 142]. Hence, the free energy for the external equilibrium reaction, $\Delta G_{r}-K_{r}=\exp \left(-\Delta G_{r} / k_{B} T\right)$-is given by

Anode: $\Delta G_{r}^{\varepsilon}=\Delta G_{r}^{o c, \varepsilon}-q \eta$

$$
\text { Cathode: } \quad \Delta G_{r}^{\delta}=\Delta G_{r}^{o c, \delta}+q \eta
$$

where $\alpha$ is the transfer coefficient $(0 \leq \alpha \leq 1)$, "oc" refers to open-circuit conditions, $\eta$ is the cell overpotential. If the interfacial potential drops are small compared to $\bar{t}_{i o n} \Phi_{t h}$ then

$$
\eta=\bar{t}_{i o n} \Phi_{t h}-\Phi_{e x t}
$$

where $\bar{t}_{i o n}$ is the average ionic transference number. Eq. (4.7.19) is substituted into Eqs. (4.7.14) through (4.7.17) to obtain potential-dependent boundary conditions for the bilayer.

\subsubsection{Results and Discussion}

\section{Open-Circuit Conditions}

We have obtained [140] expressions for $j_{V}$ based on Wagner's equation (the "thermodynamic" model) and the transport equations listed earlier (the "transport" model). Both approaches are used and the results compared below. However, we expect that the expression for $j_{V}$ derived from the transport equations [140] to be more accurate since fewer assumptions were made in its development. Nevertheless, the accuracy of both models (transport and thermodynamic) may, indirectly, be validated by comparing the results of both approaches.

“Thermodynamic” Approach. Combining Eqs. (4.7.1) with Wagner's equation (since $j_{\mathrm{O}_{2}}^{\varepsilon}=j_{\mathrm{O}_{2}}^{\delta}$ ) yields; 


$$
-j_{O_{2}}=\frac{k_{B} T}{4^{2} q^{2} L^{\varepsilon}} \cdot \int_{P_{0}}^{P_{\mathrm{O}_{2}, t}} \frac{\sigma_{V}^{\varepsilon}\left(P_{\mathrm{O}_{2}}\right) \sigma_{e}^{\varepsilon}\left(P_{\mathrm{O}_{2}}\right)}{\sigma_{V}^{\varepsilon}\left(P_{\mathrm{O}_{2}}\right)+\sigma_{e}^{\varepsilon}\left(P_{\mathrm{O}_{2}}\right)} \cdot \frac{d P}{P}=\frac{k_{B} T}{4^{2} q^{2} L^{\delta}} \cdot \int_{P_{t}}^{P_{\mathrm{O}_{2}, t}} \frac{\sigma_{V}^{\delta}\left(P_{\mathrm{O}_{2}}\right) \sigma_{h}^{\delta}\left(P_{\mathrm{O}_{2}}\right)}{\sigma_{V}^{\delta}\left(P_{\mathrm{O}_{2}}\right)+\sigma_{h}^{\delta}\left(P_{\mathrm{O}_{2}}\right)} \cdot \frac{d P_{\mathrm{O}_{2}}}{P_{\mathrm{O}_{2}}}
$$

and

$$
\tau\left(P_{\mathrm{O}_{2}, I}\right)=\frac{L^{\varepsilon} j_{\mathrm{O}_{2}}^{\varepsilon}}{L^{\delta} j_{\mathrm{O}_{2}}^{\delta}}=\frac{L^{\varepsilon}}{L^{\delta}}=\frac{\int_{P_{\mathrm{O}_{2},}}^{P_{\mathrm{O}_{2}}} \frac{\sigma_{V}^{\varepsilon}\left(P_{\mathrm{O}_{2}}\right) \sigma_{e}^{\varepsilon}\left(P_{\mathrm{O}_{2}}\right)}{\sigma_{V}^{\varepsilon}\left(P_{\mathrm{O}_{2}}\right)+\sigma_{e}^{\varepsilon}\left(P_{\mathrm{O}_{2}}\right)} \cdot \frac{d P_{\mathrm{O}_{2}}}{P_{\mathrm{O}_{2}}}}{\int_{P_{\mathrm{O}_{2}, t}}^{P_{\mathrm{O}_{2}}} \frac{\sigma_{V}^{\delta}\left(P_{\mathrm{O}_{2}}\right) \sigma_{h}^{\delta}\left(P_{\mathrm{O}_{2}}\right)}{\sigma_{V}^{\delta}\left(P_{\mathrm{O}_{2}}\right)+\sigma_{h}^{\delta}(P)} \cdot \frac{d P_{\mathrm{O}_{2}}}{P_{\mathrm{O}_{2}}}}
$$

where the conductivities, $\sigma\left(P_{\mathrm{O}_{2}}\right)$, are obtained by combining Eqs. (4.6.5) and (4.7.12) - (4.7.15).

The integral in the denominator in Eq. (4.7.23b) exists, but it is very unwieldy. For ESB, $K_{m}>$ $10^{19} \mathrm{~m}^{-3} \mathrm{~atm} .{ }^{-1 / 2}$ and $c_{A}=2.2 \times 10^{28} \mathrm{~m}^{-3}$ [140], consequently, in the range of $P_{\mathrm{O}_{2}}\left(10^{-20}\right.$ atm. to 0.21 atm. ) and $T\left(500{ }^{\circ} \mathrm{C}\right.$ to $\left.800{ }^{\circ} \mathrm{C}\right)$ under investigation

$$
8 c_{A} \gg>K_{m} P^{1 / 2}
$$

Therefore the expressions for the partial conductivities reduce to

$$
\sigma_{V}^{\delta}\left(P_{\mathrm{O}_{2}}\right)=\frac{z_{V}^{2} q^{2} D_{V}^{\delta} c_{A}^{\delta}}{2 k_{B} T}
$$

and

$$
\sigma_{h}^{\delta}\left(P_{\mathrm{O}_{2}}\right)=\frac{z_{h}^{2} q^{2} D_{h}^{\delta}}{k_{B} T} K_{m}^{\frac{1}{2}} P^{\frac{1}{4}}\left(\frac{1}{2} c_{A}^{\delta}\right)^{\frac{1}{2}}
$$

With this simplification the denominator in Eq. (4.7.20b) is easily integrated, with the result

$$
\tau\left(P_{I}\right)=\frac{\frac{z_{e}^{2} D_{e}^{\varepsilon}}{1+\frac{4}{3} \Theta^{\varepsilon}}\left\{\frac{c_{V}^{\varepsilon}(P)}{2}-\frac{\Omega^{2}}{6} \ln \frac{c_{V}^{\varepsilon}(P)-\Omega \sqrt{c_{V}^{\varepsilon}(P)}+\Omega^{2}}{\left(\sqrt{c_{V}^{\varepsilon}(P)}+\Omega\right)^{2}}-\left.\frac{\Omega^{2}}{\sqrt{3}} \tan ^{-1} \frac{2 \sqrt{c_{V}^{\varepsilon}(P)}-\Omega}{\Omega \sqrt{3}}\right|_{P_{0}} ^{P_{I}}\right\}}{-\frac{z_{h}^{2} D_{h}^{\delta} c_{A}^{\delta}}{8 \Theta^{\delta}} \cdot\left\{\ln \left(1+\Theta^{\delta}\left(\frac{1}{2} c_{A}^{\delta}\right)^{-\frac{1}{2}} K_{m}^{\frac{1}{2}} P^{\frac{1}{4}}\right)_{P_{I}}^{P_{L}}\right\}}
$$

where

$$
\Omega=-\frac{4}{3} \Theta^{\frac{1}{3}}\left(\frac{1}{2} c_{A}^{\varepsilon}\right)^{\frac{1}{2}}\left(1+\frac{4}{3} \Theta\right)^{-\frac{1}{3}}
$$




$$
\Theta^{\varepsilon, \delta}=z_{e, h}^{2} D_{e, h} /\left(z_{V}^{2} D_{V}\right)
$$

"Transport” Approach. Since $r=1$ in open-circuit conditions, then from Eq. (4.7.6) we have

$$
\tau=\frac{L^{\varepsilon}}{L^{\delta}}=\frac{D_{V}^{\varepsilon} D_{e}^{\varepsilon}}{D_{V}^{\delta} D_{h}^{\delta}} \frac{\frac{\left(z_{V}-z_{e}\right)\left(c_{V_{I}}^{\varepsilon}-c_{V_{0}}^{\varepsilon}\right)}{z_{e} D_{e}^{\varepsilon}-z_{V} D_{V}^{\varepsilon}}+\frac{z_{e}\left(D_{e}^{\varepsilon}-D_{V}^{\varepsilon}\right) c_{A}^{\varepsilon}}{\left(z_{V}-z_{h}\right)\left(c_{V_{L}}^{\delta}-z_{V}^{\delta} D_{V}^{\varepsilon}\right)^{2}} \ln \frac{z_{V}\left(z_{e} D_{e}^{\varepsilon}-z_{V} D_{V}^{\varepsilon}\right) c_{V_{I}}^{\varepsilon}-z_{e} D_{e}^{\varepsilon} c_{A}^{\varepsilon}}{z_{V}\left(z_{e} D_{e}^{\varepsilon}-z_{V} D_{V}^{\varepsilon}\right) c_{V_{0}}^{\varepsilon}-z_{e} D_{e}^{\varepsilon} c_{A}^{\varepsilon}}+\frac{z_{h}\left(D_{h}^{\delta}-D_{V}^{\delta}\right) c_{A}^{\delta}}{z_{h} D_{h}^{\delta} z_{V} D_{V}^{\delta}} \ln \frac{z_{V}\left(z_{h} D_{h}^{\delta}-z_{V} D_{V}^{\delta}\right) c_{V_{L}}^{\delta}-z_{h} D_{h}^{\delta} c_{A}^{\delta}}{z_{V}\left(z_{h} D_{h}^{\delta}-z_{V} D_{V}^{\delta}\right) c_{V_{I}}^{\delta}-z_{h} D_{h}^{\delta} c_{A}^{\delta}}}{\left.z_{V}^{\delta}\right)^{2}}
$$

\section{Results and Discussion}

The results of the model for open-circuit conditions are summarized in Table 4.7-1. Table 4.7-1 shows that there is very close agreement (enough, perhaps, to be indistinct experimentally) between the "thermodynamic" model—Eq. (4.7.23) — and the "transport" model—Eq. (4.7.26)—giving credence to the propriety of both approaches.

In the Table 4.7-1, the values for $K_{r}$ are calculated from the thermogravimetric data of Kobayashi et al. [131] and from fitting Eq. (4.6.48) to our experimental data (section 4.6). At $500{ }^{\circ} \mathrm{C}$ the value of $K_{r}$ is adjusted slightly to reflect $\bar{t}_{i o n}=0.9$ for SDC, as obtained by other researchers [145]. We expect that value wasn't achieved in our experiments due to poor electrode performance [140].

Similarly, the values for $K_{m}$ are calculated from Eq. (4.6.48) using literature values [143, 144] for $\bar{t}_{\text {ion }}\left(\Phi_{\mathrm{oc}} / \Phi_{\mathrm{th}}\right)$ and assuming $D_{e}(\mathrm{SDC}) \approx D_{h}(\mathrm{ESB})=D_{e, h}$ (therefore $\left.\Theta^{\varepsilon}=0.5 \Theta^{\delta}\right)$. In addition, $c_{A}^{\varepsilon}=5 \mathrm{x}$ $10^{27} \mathrm{~m}^{-3}, c_{A}^{\delta}=22 \times 10^{27} \mathrm{~m}^{-3}, P_{\mathrm{L}}=0.21 \mathrm{~atm}$. and $P_{0}=10^{-20}$ atm. in all calculations made in this section. Understandably, there exists some variability in the literature [143 - 145] for the value of $\bar{t}_{\text {ion }}$ and although this variability is small $(<3 \%)$ it does have a significant impact on the predicted values of $\tau$.

The ASR of the bilayered electrolyte is included in Table 4.7-1 to evaluate how low of an ASR is possible in open-circuit conditions. The total ASR of the bilayer is given by

$$
A S R_{\text {bilayer }}=\left(L^{\varepsilon} / \sigma^{\varepsilon}+L^{\delta} / \sigma^{\delta}\right)=L\left(\tau / \sigma^{\varepsilon}+1 / \sigma^{\delta}\right)(\tau+1)^{-1}
$$

where $L$ is the thickness of the bilayer $\left(L=L^{\varepsilon}+L^{\delta}\right)$ and $\sigma$ is the average conductivity. For large $\tau$ (i.e., $\tau>\sim 2$ at $800{ }^{\circ} \mathrm{C}$ and $\tau>\sim 3$ at $500{ }^{\circ} \mathrm{C}$ ) Eq. (4.7.27) reduces to $L / \sigma^{\varepsilon}$-i.e., the ASR depends solely on the conductivity of SDC. For small $\tau$ (i.e., $\tau<\sim 0.02$ at $800{ }^{\circ} \mathrm{C}$ and $\tau<\sim 0.03$ at $500{ }^{\circ} \mathrm{C}$ ) Eq. (4.7.27) reduces to $L / \sigma^{\delta}$-i.e., the ASR depends solely on the conductivity of ESB. 
Table 4.7-1. $\tau^{*}$ and ASR $(\mathrm{L}=10 \mu \mathrm{m})$ at $800^{\circ} \mathrm{C}$ and $500^{\circ} \mathrm{C}$ in open-circuit conditions.

\begin{tabular}{|c|c|c|c|c|c|c|c|c|}
\hline $\begin{array}{c}T \\
\left({ }^{\circ} \mathrm{C}\right)\end{array}$ & $\begin{array}{c}K_{r} \\
\left(\mathrm{~m}^{-}\right. \\
\left.{ }_{9} \mathrm{~atm}^{1 / 2}\right)\end{array}$ & $\begin{array}{c}K_{m} \\
\left(\mathrm{~m}^{-3} \mathrm{~atm} .^{-}\right. \\
1 / 2)\end{array}$ & $\Theta_{\mathrm{SDC}}\left(\Theta_{\mathrm{ESB}}\right)$ & $\begin{array}{c}\bar{t}_{\text {ion }} \\
(\mathrm{SDC})\end{array}$ & $\begin{array}{c}\bar{t}_{\text {ion }} \\
(\mathrm{ESB})\end{array}$ & $\tau^{*}$ thermo & $\tau_{\text {transpor }}^{*}$ & $\begin{array}{c}\mathrm{ASR}^{\dagger \dagger} \\
\left(\Omega \mathrm{cm}^{2}\right)\end{array}$ \\
\hline 800 & $1.0 \times 10^{72}$ & $2.0 \times 10^{23}$ & $\$ 11.5(5.75)$ & 0.80 & 0.98 & 17.2 & 17.8 & 0.010 \\
\hline 800 & $1.0 \times 10^{72}$ & $8.4 \times 10^{23}$ & $\$ 11.5(5.75)$ & 0.80 & 0.96 & 8.5 & 8.8 & 0.010 \\
\hline 800 & ${ }^{*} 1.4 \times 10^{79}$ & $2.0 \times 10^{23}$ & $\ddagger 0.35(5.75)$ & 0.75 & 0.98 & 18000 & 24000 & 0.011 \\
\hline 800 & $\$ 1.4 \times 10^{79}$ & $8.4 \times 10^{23}$ & $\star_{0.35(5.75)}$ & 0.75 & 0.96 & 9100 & 12000 & 0.011 \\
\hline 500 & $\$ 4.0 \times 10^{65}$ & $1.0 \times 10^{18}$ & $\$ 2570(1285)$ & 0.84 & 0.99 & 9.8 & 9.8 & 0.12 \\
\hline 500 & $\$ 4.0 \times 10^{65}$ & $9.3 \times 10^{18}$ & $\$ 2570(1285)$ & 0.84 & 0.97 & 3.2 & 3.2 & 0.11 \\
\hline 500 & $5.1 \times 10^{64}$ & $1.0 \times 10^{18}$ & $\$ 2570(1285)$ & 0.90 & 0.99 & 4.6 & 4.6 & 0.12 \\
\hline 500 & $5.1 \times 10^{64}$ & $9.3 \times 10^{18}$ & $\$ 2570(1285)$ & 0.90 & 0.97 & 1.53 & 1.53 & 0.10 \\
\hline $\begin{array}{l}\text { Calcul } \\
\text { Obtain } \\
\text { ASR, }\end{array}$ & from thermo & vimetric data & Kobayashi et al & 31] sec & $4.6, \mathrm{~T}$ & $\begin{array}{l}4.6-2 . \\
4.6-4 . \\
C ; 0.04\end{array}$ & & \\
\hline
\end{tabular}

Using $K_{r}$ calculated from the data of Kobayashi et al. [131], the optimal (minimum) thickness ratio, $\tau^{*}$, to prevent decomposition of the ESB layer-ESB decomposes for $P_{\mathrm{O}_{2}, I} \leq \sim 10^{-9}$ atm. [143, $144]$ at $800{ }^{\circ} \mathrm{C}$ - at the SDC-ESB interface, is $\sim 9$ or $\sim 18$ depending on whether $\bar{t}_{\text {ion }}$ is 0.98 or 0.96 for ESB.

Samaria-doped ceria is more electronically conductive than ESB and so it permeates oxygen more easily. If the layers had the same thickness, the chemical potential drop would be greater across the more resistive layer (analogous to an electrical circuit). Consequently, to increase the chemical potential drop across the SDC layer and offer protection to the ESB layer requires a thickening of the SDC layer relative to the ESB layer. Accordingly, if the ESB layer is more permeable (i.e., smaller $\left.\bar{t}_{i o n}\right)$ then the chemical potential drop across it is smaller and a thinner layer of SDC (smaller $\tau^{*}$ ) can provide the same protection. By extension, this suggests that there is a trade-off between having a bilayer that produces near theoretical open-circuit potential and one that has the highest conductivity (due to the thinner SDC layer).

At $800{ }^{\circ} \mathrm{C}$ the ASR (for $\mathrm{L}=10 \mu \mathrm{m}$ ) of the bilayered electrolyte is approximately equal to that of a single-layer SDC electrolyte (i.e., the ESB layer has a negligible effect on the ASR of the bilayer). (At $800{ }^{\circ} \mathrm{C}$, the ASR is $0.011 \Omega \mathrm{cm}^{2}$ and $0.0024 \Omega \mathrm{cm}^{2}$ for SDC and ESB respectively.) This result is a consequence of the high $\tau^{*}$ values necessitated by the oxygen permeability of SDC and though this result is not ideal, it is still has promise because the ESB layer blocks conduction of electrons through 
the bilayer and, thereby should increase the efficiency of power delivery to an external load compared to SDC alone.

The results obtained using $K_{r}$ and $\Theta$ calculated from our experimental data for SDC at $800{ }^{\circ} \mathrm{C}$, section 4.6, reflects the difficulties we experienced with the Pt-paste electrodes and emphasizes the need to separate electrode effects from the measured value of $\Phi_{\mathrm{oc}}$ in order to accurately determine the performance of the electrolyte. Although more reasonable, the $K_{r}$ and $\Theta$ values calculated from our experimental data at $500{ }^{\circ} \mathrm{C}$ likewise suffer from electrode effects, in this case evidenced in the lower than expected $\bar{t}_{i o n}$.

At $500{ }^{\circ} \mathrm{C}$, ESB decomposes at $P_{I} \leq \sim 10^{-16}$ atm. $[143,144]$ and $\tau^{*}$ ranges from $\sim 1.5$ to $\sim 10$ depending on whether $\bar{t}_{i o n}$ is 0.84 or 0.90 for SDC and 0.98 or 0.96 for ESB. Again this value is approximately equal to that of a single-layer SDC electrolyte alone. (At $500{ }^{\circ} \mathrm{C}$, the ASR is $0.13 \Omega \mathrm{cm}^{2}$ and $0.048 \Omega \mathrm{cm}^{2}$ for SDC and ESB respectively.)

Calculating $\tau^{*}$ from the most conservative estimates of $K_{r}$ and $K_{m}$ (or $\bar{t}_{\text {ion }}$ ) will result in a very low ASR of $\sim 0.01$ at $800{ }^{\circ} \mathrm{C}$ and an acceptably low ASR of $\sim 0.1$ at $500{ }^{\circ} \mathrm{C}$. Either ASR would enable a high power density SOFC if electrodes with suitable performance (i.e., low overpotential) were found.

\section{Closed-Circuit Conditions}

At $800{ }^{\circ} \mathrm{C}$ and $500{ }^{\circ} \mathrm{C}$, ESB decomposes in a $\sim 0.44 \mathrm{~V}$ and $\sim 0.62 \mathrm{~V}$ potential difference $\left(\Phi_{\text {ext }}\right)$ relative to air, respectively $[143,144]$. So, if the potential difference across the ESB layer is held below $0.4 \mathrm{~V}$ at $800{ }^{\circ} \mathrm{C}$ and $0.6 \mathrm{~V}$ at $500{ }^{\circ} \mathrm{C}$, then the ESB layer remains stable at those temperatures. Additionally, it can be shown that the decomposition potential and the Galvani potential, $\Delta \phi$, are identical. The operating conditions for the bilayer electrolyte SOFC are listed in Table 4.7-2 $\left(P_{\mathrm{O}_{2}, \mathrm{~L}}=\right.$

Table 4.7-2. Constants used in generating results from the bilayer model.

\begin{tabular}{ccccccc}
\hline $\begin{array}{c}\text { Temperature } \\
\left({ }^{\circ} \mathrm{C}\right)\end{array}$ & $\begin{array}{c}P_{\mathrm{O}_{2}, 0} \\
(\mathrm{~atm} .)\end{array}$ & $\begin{array}{c}K_{r} \\
\left(\mathrm{~m}^{-9} \mathrm{~atm} .^{1 / 2}\right)\end{array}$ & $\begin{array}{c}K_{m} \\
\left(\mathrm{~m}^{-3} \mathrm{~atm}^{-1 / 2}\right)\end{array}$ & $\begin{array}{c}D_{V}^{\varepsilon} \\
\left(\mathrm{cm}^{2} / \mathrm{s}\right)^{\dagger}\end{array}$ & $\begin{array}{c}D_{V}^{\delta} \\
\left(\mathrm{cm}^{2} / \mathrm{s}\right)^{\S}\end{array}$ & $\begin{array}{c}D_{e}^{\varepsilon}=D_{h}^{\delta} \\
\left(\mathrm{cm}^{2} / \mathrm{s}\right)^{\dagger}\end{array}$ \\
\hline 800 & $10^{-20}$ & $10^{72}$ & $10^{23}$ & $5.5 \times 10^{-6}$ & $5.4 \times 10^{-6}$ & $2.2 \times 10^{-4}$ \\
500 & $10^{-24}$ & $7 \times 10^{66}$ & $4 \times 10^{20}$ & $2.2 \times 10^{-6}$ & $2.0 \times 10^{-6}$ & $7.3 \times 10^{-5}$ \\
\hline
\end{tabular}

${ }^{\dagger}$ Steele [145]; ${ }^{\S}$ Shuk et. al. [146] 

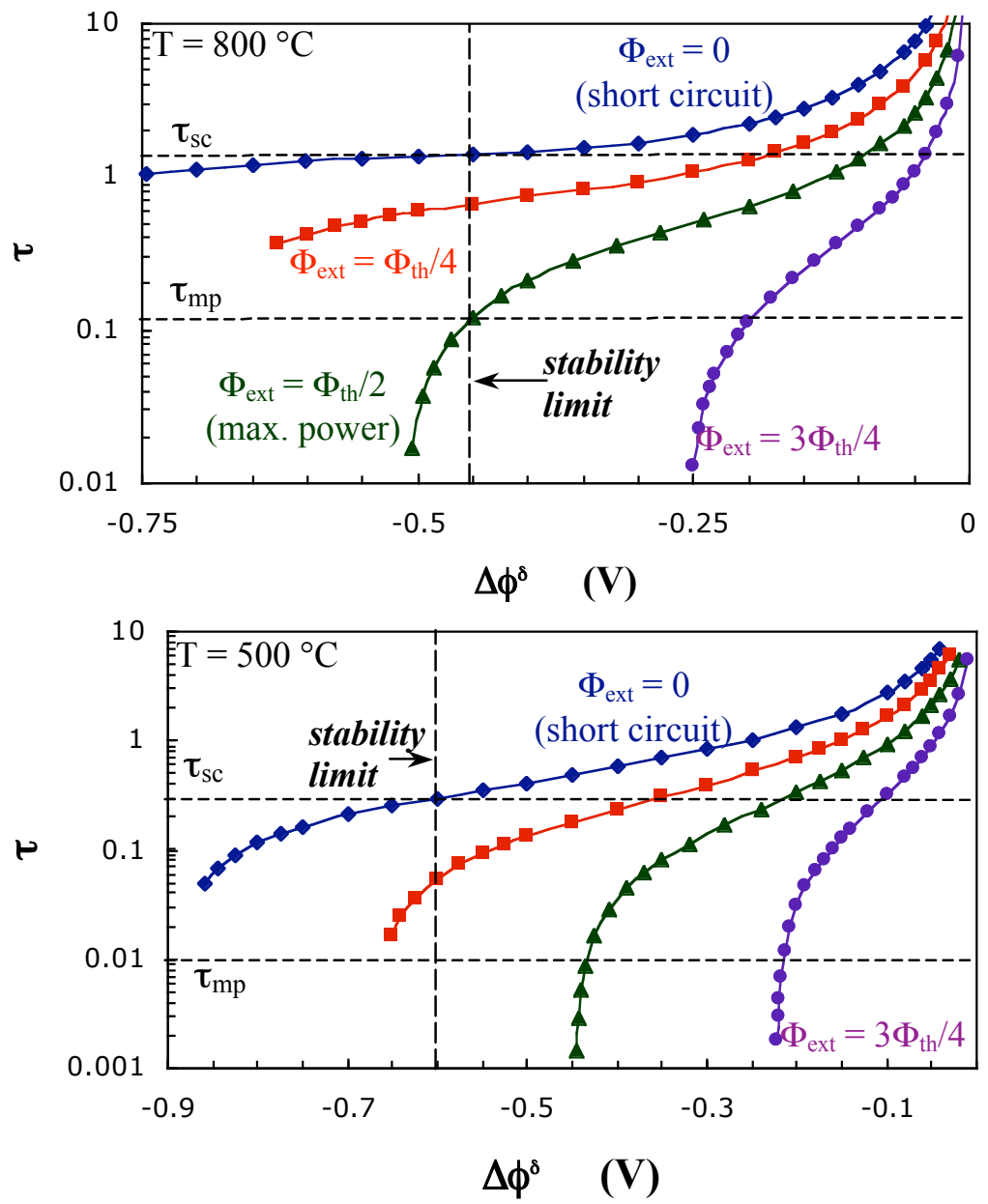

Figure 4.7.2. Thickness ratio $\left(\tau=L^{\varepsilon} / L^{\delta}\right)$ required to obtain desired Galvani potential drops across the ESB layer of the bilayer at (a) $800{ }^{\circ} \mathrm{C}$ and (b) $500{ }^{\circ} \mathrm{C}$ in various operating conditions.

$0.21 \mathrm{~atm}$ for both temperatures). The values for the equilibrium constants, $K_{r}$ and $K_{m}$, were obtained from fitting open-circuit voltage data and thermogravimetric data [140]. Values for $D_{V}^{\varepsilon}$ and $D_{V}^{\delta}$ were obtained from reviews by Steele [145] and Shuk et al [146] respectively. Finally, in the absence of reliable data for $D_{h}^{\delta}$, we assumed that $D_{e}^{\varepsilon}=D_{h}^{\delta}$ (where $D_{e}^{\varepsilon}$ was obtained from Steele [145]). This assumption should not incur significant error since ESB and SDC are both fluorite-structured crystals.

The results of modeling the SDC $\backslash E S B$ bilayer are shown in Figs. 4.7.2 through 4.7.5. They were obtained from numerical solutions of Eqs. (4.7.9) and (4.7.10) the results of which were put into Eqs. (4.7.5), (4.7.6) and (4.7.13) to generate the plots. In Fig. 4.7.2 are plots, at $800{ }^{\circ} \mathrm{C}$ and $500^{\circ} \mathrm{C}$, of the thickness ratio, $\tau$, against the Galvani potential drop across the ESB layer, $\Delta \phi^{\delta}$, for four SOFC operating conditions: $\Phi_{\text {ext }}=0$ (short-circuit), $\Phi_{\text {ext }}=\Phi_{t h} / 4, \Phi_{\text {ext }}=\Phi_{t h} / 2$ (maximum power) and $\Phi_{\text {ext }}=$ $3 \Phi_{t h} / 4$. The graphs show that the value of $\tau$ chosen determines the range of operating conditions 

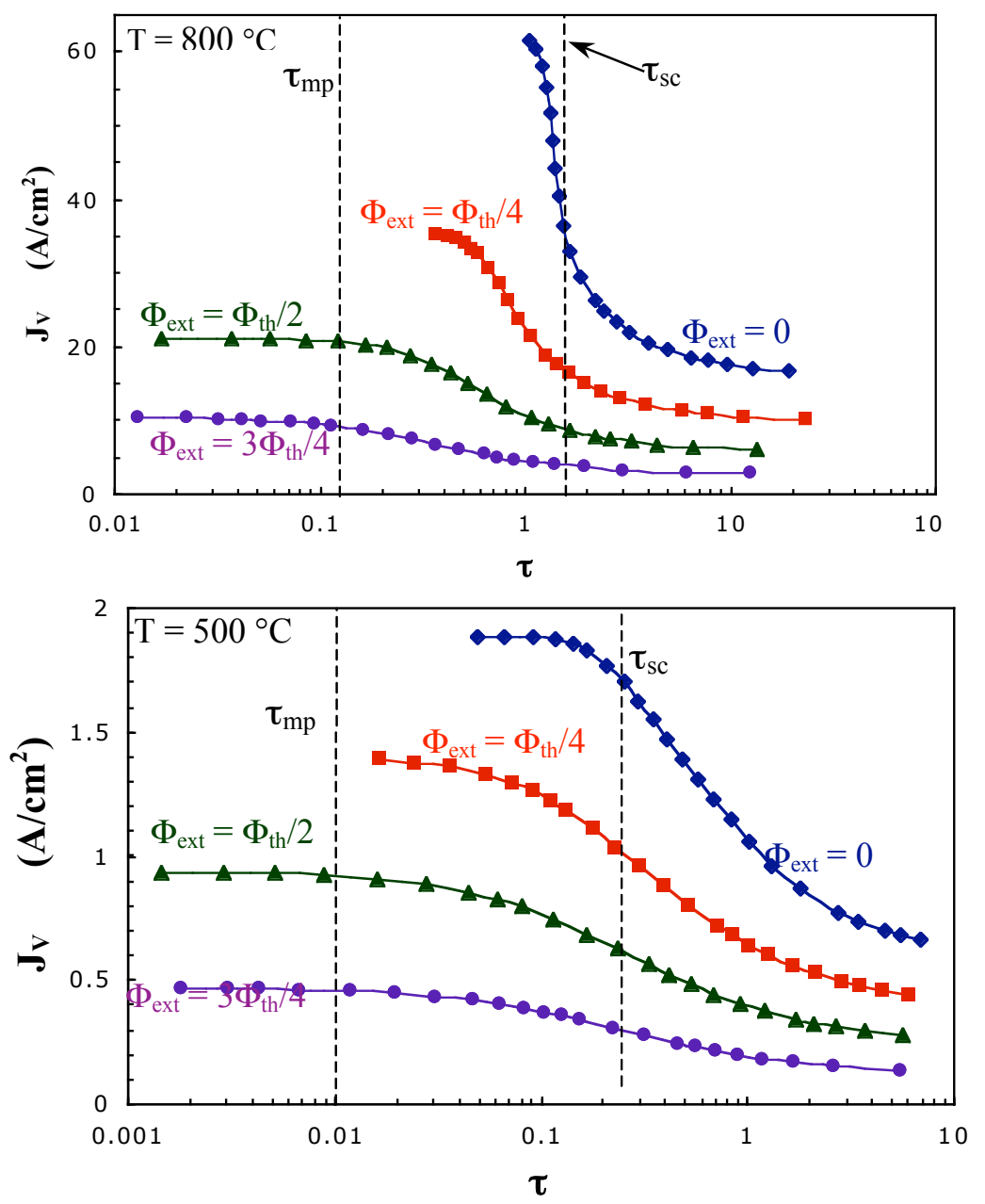

Figure 4.7.3. Dependence of ionic current density on thickness ratio $\left(\tau=L^{\varepsilon} / L^{\delta}\right)$ for a $100 \mu \mathrm{m}$ thick bilayer electrolyte at (a) $800{ }^{\circ} \mathrm{C}$ and (b) $500{ }^{\circ} \mathrm{C}$ in various operating conditions.

acceptable to maintain stability of the bilayer electrolyte. Thus, at $800{ }^{\circ} \mathrm{C}$, Fig. $4.7 .2 \mathrm{a}$, the bilayer electrolyte is stable in the range $\Phi_{\mathrm{oc}}<\Phi_{\text {ext }} \leq 0$ (short-circuit) for $\tau \geq 1.4\left(\tau_{\mathrm{sc}}\right)$. However, a more favorable (thicker ESB/thinner SDC) value of $\tau=0.12\left(\tau_{\mathrm{mp}}\right)$ becomes acceptable if the SOFC operates in the range: $\Phi_{\mathrm{oc}}<\Phi_{\mathrm{ext}} \leq \Phi_{\mathrm{th}} / 2$ (maximum power).

Even better results are predicted for $500{ }^{\circ} \mathrm{C}$, Fig. 4.7.2b, where the bilayer electrolyte should be stable up to short-circuit conditions for $\tau \geq 0.3\left(\tau_{\mathrm{sc}}\right)$. Figure 4.7.2b further shows that $\Delta \phi^{\delta}$ never gets as high as $0.6 \mathrm{~V}$ (the ESB decomposition potential at $500^{\circ} \mathrm{C}$ ) if the SOFC operates in the range: $\Phi_{\text {oc }}<$ $\Phi_{\mathrm{ext}} \leq \Phi_{\mathrm{th}} / 2$ (maximum power). This occurs because $\Phi_{\mathrm{th}} / 2<0.6 \mathrm{~V}$. Therefore, in principle, we can choose $\tau$ to be vanishingly small for those conditions. However, for the sake of prudence, a conservative value of $\tau=0.01\left(\tau_{\mathrm{mp}}\right)$ is used for the remainder of the discussion. 

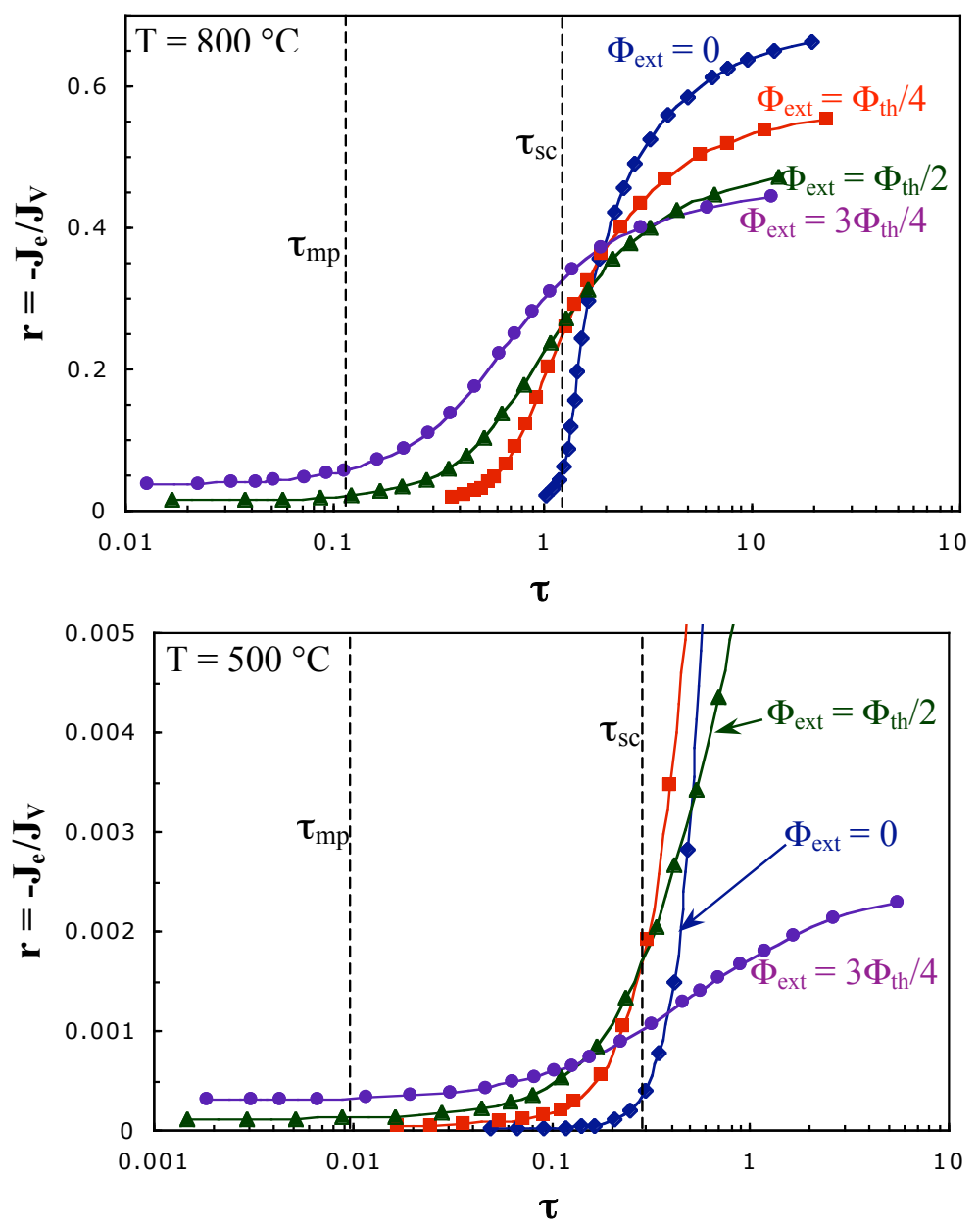

Figure 4.7.4. Dependence of the electronic-ionic current ratio on thickness ratio $\left(\tau=L^{\varepsilon} / L^{\delta}\right)$ for a 100 $\mu \mathrm{m}$ thick bilayer electrolyte at (a) $800^{\circ} \mathrm{C}$ and (b) $500^{\circ} \mathrm{C}$ in various operating conditions.

Figure 4.7.3 shows how the ionic current density varies with thickness ratio and operating conditions for a total bilayer thickness of $100 \mu \mathrm{m}$. As expected, $J_{V}$ increases as $\tau$ decreases (more ESB or less SDC). Also, the effect of the ESB layer (higher conductivity) on $J_{V}$ becomes more pronounced as $\Phi_{\text {ext }}$ approaches zero (i.e., short-circuit) and as $T$ increases. However, perhaps the most salient feature of the graphs, is the appreciable increase in $J_{V}$, even at the larger $\left(\tau_{\mathrm{sc}}\right)$ of the two $\tau$ values considered at each temperature. Finally, the predicted current density values far exceed current goals for SOFCs.

Figure 4.7.4 shows how the electronic-ionic current ratio, $r$, which is a measure of the quality of the electrolyte, varies with thickness ratio and operating conditions. As expected, $r$ decreases (i.e., the bilayer becomes a better electrolyte) with decreasing $\tau$. Like $J_{V}$ in Fig. 4.7.3, this trend becomes sharper 


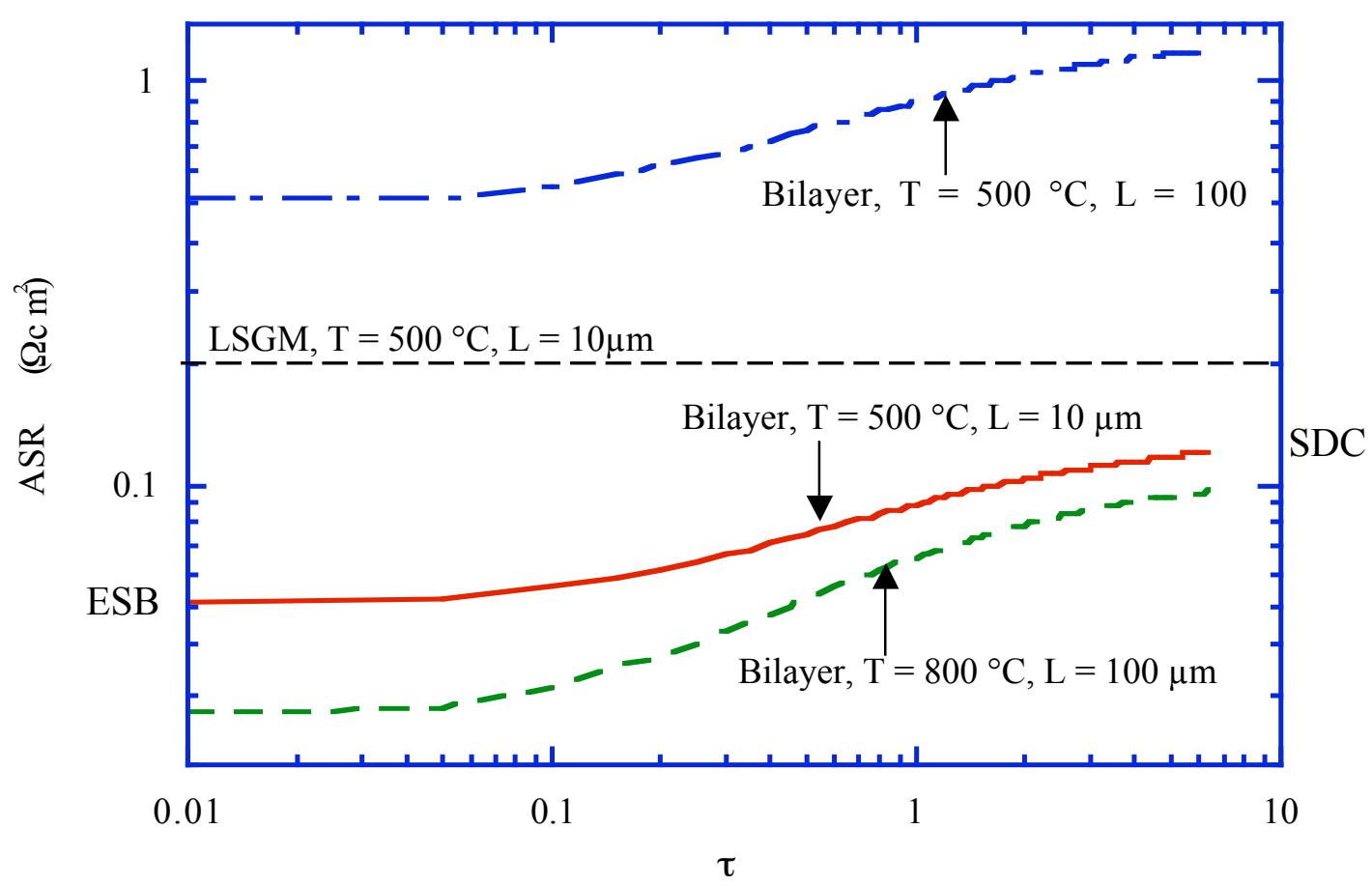

Figure 4.7.5. ASR as a function of relative thickness, total thickness and temperature.

as $\Phi_{\text {ext }}$ approaches zero and $\mathrm{T}$ increases. More significant is the marked improvement (i.e., decrease) in $r$ that can be achieved even when the SDC layer is thicker than the ESB layer $(\tau>1)$.

Figure 4.7.5 shows a plot of the projected ASR for an SDC-ESB bilayer at different temperatures and total thickness. The SDC and ESB conductivity values used were determined in air $[145,146]$ so the effect of an oxygen potential gradient and electronic conductivity in SDC are neglected, this is a significant simplification at high temperature $\left(800{ }^{\circ} \mathrm{C}\right)$ [145]. However, the significance of these factors should diminish with decreasing temperature. At small $\tau$ the bilayer ASR approaches that of ESB, at large $\tau$ it approaches that of SDC. Figure 4.7.5 shows that, in principle, an SDC-ESB bilayer should be able to approach the target ASR value of $0.1-0.2 \Omega \mathrm{cm}^{2}$. Moreover, at $500{ }^{\circ} \mathrm{C}$, the bilayer ASR is notably less than the ASR for any alternative electrolyte, SDC or LSGM, especially as $\tau$ decreases (i.e., more ESB less SDC).

Figure 4.7.6 shows the dependence of the area-specific resistance, ASR, on thickness ratio and operating conditions for the bilayer and the ESB layer alone. The ASR was calculated from the formula below

$$
A S R_{\text {bilayer }}=\left(L^{\varepsilon} / \bar{\sigma}^{\varepsilon}+L^{\delta} / \bar{\sigma}^{\delta}\right)=L\left(\tau / \bar{\sigma}^{\varepsilon}+1 / \bar{\sigma}^{\delta}\right)(\tau+1)^{-1}
$$



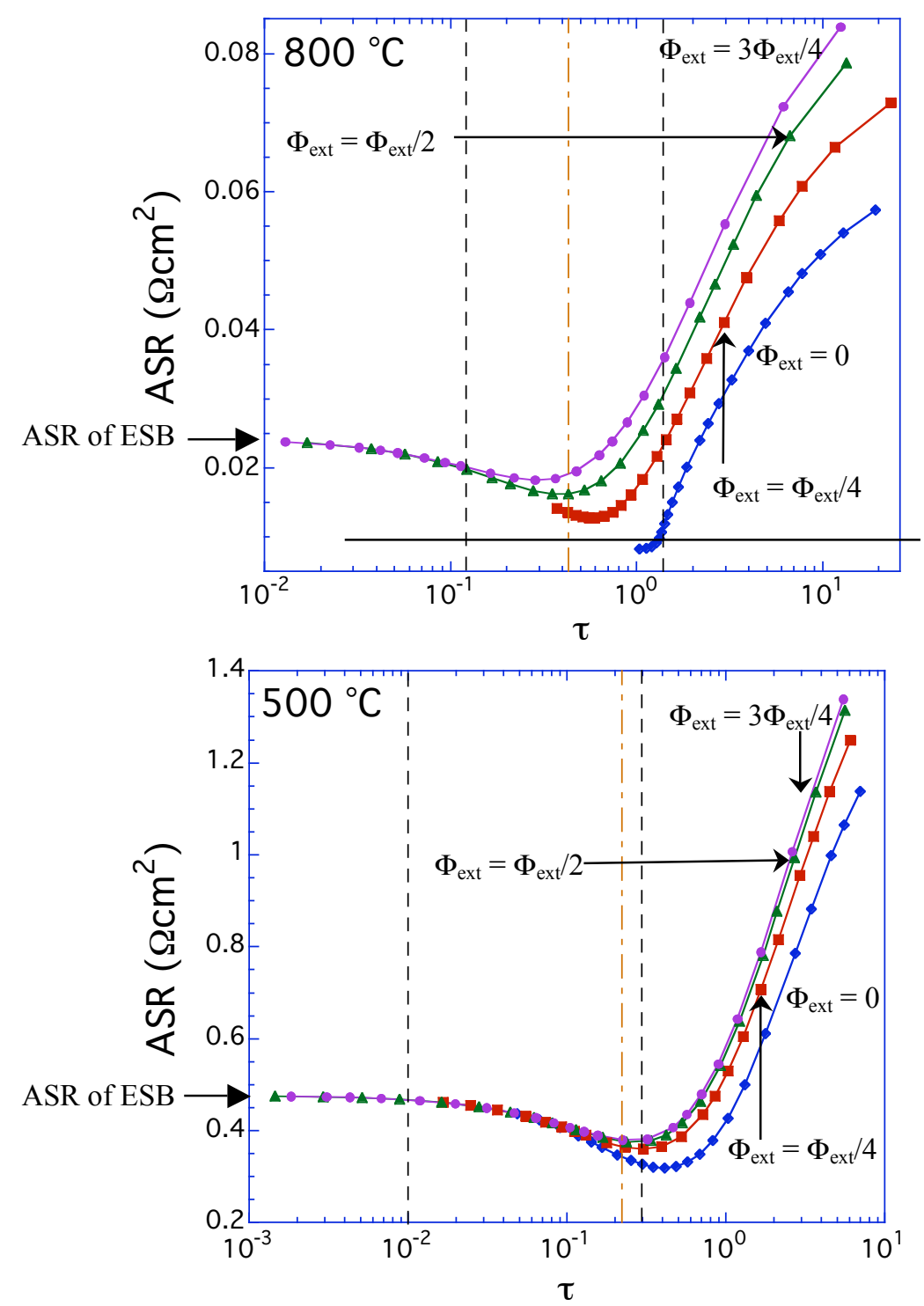

Figure 4.7.6. Dependence of the area-specific resistance on thickness ratio $\left(\tau=L^{\varepsilon} / L^{\delta}\right)$ for a $100 \mu \mathrm{m}$ thick bilayer electrolyte at (a) $800{ }^{\circ} \mathrm{C}$ and (b) $500{ }^{\circ} \mathrm{C}$ in various operating conditions.

where $L$ is the thickness of the bilayer $\left(L=L^{\varepsilon}+L^{\delta}\right)$ and $\bar{\sigma}$ is the average conductivity (average values are used in the presence of concentration gradients to take into consideration the change in $\sigma$ with $P_{\mathrm{O}_{2}}$ ) such that $[136,139,140]$ :

$$
\bar{\sigma}_{i}^{-1}=\left(-z_{i} q J_{i} L\right)^{-1}\left[k_{B} T \int_{c_{i_{0}}}^{c_{i_{L}}} c_{i}^{-1} \cdot \mathrm{d} c_{i}+z_{i} q \int_{\phi_{0}}^{\phi_{L}} \mathrm{~d} \phi\right]=\left(-z_{i} q J_{i} L\right)^{-1}\left[k_{B} T \ln \left(c_{i_{L}} / c_{i_{0}}\right)+z_{i} q \Delta \phi\right]
$$

Without concentration gradients, Eq. (4.7.29) reduces to the usual definition for conductivity.

The ASR of a mixed conductor, like SDC, is influenced appreciably by the operating conditions as a result of their effect on the boundary values of the MIEC. Figure 4.7.6 shows that this effect is 
also seen in the bilayer because of the presence of SDC. In other words, since the ASR of mixed conducting SDC depends on the applied potential, section 4.6, so does the ASR of the bilayer, especially for $\tau>1$. As $\tau$ approaches zero, this effect is reduced because the bilayer is dominated by ESB. Figure 4.7.6b shows that the effect becomes less pronounced at $500{ }^{\circ} \mathrm{C}$ where SDC displays less mixed conducting behavior.

In Fig. 4.7.6, the bilayer ASR approaches the ESB ASR as $\tau$ decreases and becomes equal to it at low values of $\tau$. In doing so, however, the bilayer ASR goes through a local minimum. This is readily explained by considering the conductance of each layer. As the relative thickness of the SDC layer decreases, eventually it will have a higher conductance than the ESB layer because the higher conductivity of the ESB layer is mitigated by its greater relative thickness. Thus, there exists an optimal $\tau$ at which the total conductance of the bilayer is a maximum, and the ASR is a minimum (for a fixed total thickness).

The most noteworthy consequence of this result is that the bilayer ASR minima occur at values larger than the lowest feasible values of $\tau$. In fact they all fall between $\tau_{\mathrm{sc}}$ and $\tau_{\mathrm{mp}}$. Since SOFC performance in maximum power delivery is the most vital criteria, we selected thickness ratio that corresponds to the minimum ASR value for $\Phi_{\text {ext }}=\Phi_{\mathrm{th}} / 2$ as the optimal thickness ratio, $\tau_{\mathrm{opt}}$. At $800{ }^{\circ} \mathrm{C}$, $\tau_{\mathrm{opt}} \approx 0.43$ and at $500{ }^{\circ} \mathrm{C}, \tau_{\mathrm{opt}} \approx 0.24$. In addition, Figs. 4.7.3 and 4.7.4 show that not much improvement in $J_{V}$ or $r$ is obtained for $\tau<\tau_{\text {opt, }}$, while Fig. 4.7.2 shows that the range of stable operation for the bilayer is extended appreciably for $\tau=\tau_{\mathrm{opt}}$ compared to $\tau=\tau_{\mathrm{mp}}$.

The most pertinent results of those presented in this section are summarized in Table 4.7-3, which shows that very low ASR values of $0.002 \Omega \mathrm{cm}^{2}$ at $800{ }^{\circ} \mathrm{C}\left(\tau_{\mathrm{mp}}=0.12\right)$ and $0.045 \Omega \mathrm{cm}^{2}$ at 500 ${ }^{\circ} \mathrm{C}\left(\tau_{\mathrm{mp}}=0.01\right)$ are attainable for bilayer electrolytes of total thickness $10 \mu \mathrm{m}$ when optimized to deliver maximum power.

Moreover, as Table 4.7-4 shows, the ASR of the bilayer compares favorably to YSZ-ASR $\sim 2.2$ $\Omega \mathrm{cm}^{2}$ — and to $\mathrm{La}_{0.8} \mathrm{Sr}_{0.2} \mathrm{Ga}_{0.8} \mathrm{Mg}_{0.2} \mathrm{O}_{3-\delta}$ (LSGM) - ASR $\sim 0.2 \Omega \mathrm{cm}^{2}$ — at $500{ }^{\circ} \mathrm{C}$ for a $10 \mu \mathrm{m}$

Table. 4.7-3. Summary of results for bilayer.

\begin{tabular}{ccccc}
\hline & $\tau$ & $\mathrm{ASR}\left(\Omega \mathrm{cm}^{2}\right)$ for $\mathrm{L}=100 \mu \mathrm{m}$ & $\mathrm{ASR}\left(\Omega \mathrm{cm}^{2}\right)$ for $\mathrm{L}=10 \mu \mathrm{m}$ & $r$ \\
\hline \multirow{2}{*}{$800^{\circ} \mathrm{C}$} & $\tau_{\mathrm{mp}}=0.12$ & 0.02 & 0.002 & 0.02 \\
& $\tau_{\mathrm{sc}}=1.4$ & 0.01 & 0.001 & 0.05 \\
\hline \multirow{2}{*}{$500^{\circ} \mathrm{C}$} & $\tau_{\mathrm{mp}}=0.01$ & 0.45 & 0.045 & 0.0002 \\
& $\tau_{\mathrm{sc}}=0.3$ & 0.30 & 0.030 & 0.0005 \\
\hline
\end{tabular}


Table. 4.7-4. Comparison of ASR for candidate SOFC electrolytes at $500{ }^{\circ} \mathrm{C}$.

\begin{tabular}{ccc}
\hline Electrolyte & $\operatorname{ASR}\left(\Omega \mathrm{cm}^{2}\right) ; \mathrm{L}=100 \mu \mathrm{m}$ & $\mathrm{ASR}\left(\Omega \mathrm{cm}^{2}\right) ; \mathrm{L}=10 \mu \mathrm{m}$ \\
\hline YSZ & 21.74 & 2.17 \\
LSGM & 1.96 & 0.20 \\
GDC & 1.05 & 0.11 \\
SDC\ESB $\left(\tau=\tau_{\mathrm{mp}}=0.01\right)$ & 0.45 & 0.05 \\
ESB & 0.48 & 0.05 \\
DWSB & 0.23 & 0.02 \\
\hline
\end{tabular}

electrolyte thickness. Using our newly discovered DWSB (section 4.2) would reduce the ASR to 0.02 $\Omega \mathrm{cm}^{2}$. With an ASR that is one order of magnitude smaller than LSGM and two orders of magnitude smaller than YSZ, the bilayer electrolyte is the only viable candidate for low temperature operation.

\subsubsection{Conclusion}

The feasibility of a bilayer electrolyte consisting of a layer of SDC on the fuel (reducing side) side and ESB on the air (oxidizing) side of an SOFC has been studied. Expressly, the feasibility of the bilayer electrolyte depends on the optimization of the thickness ratio of the layers so that the ESB layer is protected from decomposition while the ionic conductance of the combination is maximized. We have shown that this is possible and results in an electrolyte with high current density, low leakage current (small $r$ ) and low ASR even at $500{ }^{\circ} \mathrm{C}$. Accordingly, if a bilayer based fuel cell is to be operated between open-circuit and maximum power conditions (which is typical), then, for operation at $800{ }^{\circ} \mathrm{C}$, the optimal value of the thickness ratio, $\tau\left(\mathrm{L}_{\mathrm{SDC}} / \mathrm{L}_{\mathrm{ESB}}\right)$, is $\sim 0.1$ with an ASR of $0.002 \Omega \mathrm{cm}^{2}$ for a $10 \mu \mathrm{m}$ thick bilayer. Likewise, at $500{ }^{\circ} \mathrm{C}$ the optimal $\tau$ is $\sim 0.01$ with an ASR of $0.05 \Omega \mathrm{cm}^{2}$. This is an excellent result because it means there will be more bismuth oxide (higher conductivity) in the bilayer than ceria.

Finally, a bismuth oxide/ceria bilayer electrolyte was tested and it was shown to provide near theoretical open-circuit potential and remained stable for 1400 hours of fuel cell operation under both open-circuit and maximum power conditions [147]. 


\section{PROJECT SUMMARY AND FORECAST}

In this section, the accomplishments of the project on bilayered electrolytes are summarized and a forecast the potential of SOFCs based on bilayered electrolytes is detailed.

\subsection{Experimental}

\section{Fabrication}

A Colloidal deposition technique was developed to fabricate anode-supported SOFCs with thin film GDC single-layer electrolytes and GDC $\backslash$ ESB bilayer electrolytes. The single-layer cells produced power densities and I-V characteristics comparable with those reported (for ceria-based electrolytes) in the literature.

A new bismuth based electrolyte, Dysprosium- and Tungsten stabilized Bismuth oxide (DWSB) was developed. This family of stabilized bismuth oxides has the highest reported oxide-ion conductivity among known (phase stable) solid electrolytes, approximately twice that of ESB (the former holder of that title). They have not been employed in a ceria/bismuth oxide bilayer configuration yet, but they have the potential to reduce the conductance of the bilayer even further, leading to even higher power densities for a given temperature.

\section{Characterization}

Ceria/bismuth oxide bilayers were characterized in a number of ways to explore their properties. From our SEM studies we found no visual evidence of the formation of secondary phases at the ceriabismuth oxide interface. This was further corroborated when X-ray line scans and XPS readings were made of the bilayer samples. Moreover, XRD patterns of the bilayer showed that only the fluorite phase (native to both ceria and $\delta$-phase stabilized bismuth oxide) was present.

While it is possible to perform more sensitive analysis through other characterization techniques, it was deemed unnecessary for two reasons. First, solid solutions of ceria in bismuth oxide or bismuth oxide in ceria have conductivities comparable to the host/solvent. Second, our results (section 4.3) showed that the addition of a bismuth oxide layer increased the effective conductivity compared to a single layer of samaria-doped ceria alone. 
In tests of the open-circuit voltage, it was shown that increasing the thickness of the bismuth oxide layer relative to the ceria layer significantly improved the open-circuit voltage (up to near theoretical values) of the bilayer electrolytes in a temperature range from $500{ }^{\circ} \mathrm{C}$ to $800{ }^{\circ} \mathrm{C}$.

The current-voltage (I-V) characteristics and power density curves of an SOFC with a bilayer electrolyte were not determined before the project came to an end. However, extrapolations based on our thin film ceria based SOFC show that because the electron/hole blocking bismuth oxide layer raises the open-circuit potential (compared to ceria alone), large improvements in the power density curves should be accrued. Moreover, if low polarization cathodes such as the $\mathrm{Ag} / \mathrm{Bi}_{2} \mathrm{O}_{3}$ composite cathode developed by Xia et. al. [148], are used in the bilayer SOFC even higher performance will be attained.

Table 5-1 summarizes the projected performance of (Ni/GDC) anode-supported SOFC with thin film GDC $\backslash E S B$ electrolyte and Ag/YSB cathode [148] at various temperatures. Anode polarization would be a minor contribution for an anode supported SOFC (at least with a $\mathrm{H}_{2}$ fuel) and was ignored. The projected maximum power densities are considerably higher than obtained with known electrolytes. The projected maximum power density at $800{ }^{\circ} \mathrm{C}$ is an order of magnitude greater than possible with the best YSZ electrolytes. At $500{ }^{\circ} \mathrm{C}$ the maximum power density is comparable to that attained with YSZ SOFCs, using state of the art cathodes, at $600^{\circ} \mathrm{C}$ [149]. The significance of this result increases when the exponential drop in electrode performance, as temperature decreases, is taken into consideration for our projected performance.

As can be seen from the table, with our electrolyte the cell polarization is completely dominated by the cathode ASR (it is one to two orders of magnitude greater than that of the electrolyte). The $\mathrm{Ag} / \mathrm{YSB}$ cathodes are presently among the best available [148], but further improvement of cathode polarization combined with the bilayer electrolyte could push SOFC operating temperatures below $500^{\circ} \mathrm{C}$.

Table 5-1. Projected performance of (Ni/GDC) anode-supported SOFC with a $10 \mu \mathrm{m}$ thick GDC $\backslash \mathrm{ESB}$ electrolyte and $\mathrm{Ag} / \mathrm{YSB}$ cathode [148] at various temperatures.

\begin{tabular}{cccccc}
\hline $\begin{array}{c}\text { Temperature } \\
\left({ }^{\circ} \mathrm{C}\right)\end{array}$ & $\begin{array}{c}\text { ESB Decomposition } \\
\text { Voltage }(\mathrm{V})\end{array}$ & $\begin{array}{c}\tau_{\mathrm{sc}} \\
\begin{array}{c}\text { Cathode ASR } \\
\left(\Omega \mathrm{cm}^{2}\right)\end{array}\end{array}$ & $\begin{array}{c}\text { Bilayer ASR } \\
\left(\Omega \mathrm{cm}^{2}\right)\end{array}$ & $\begin{array}{c}\text { Maximum Power } \\
\text { Density }\left(\mathrm{W} / \mathrm{m}^{2}\right)\end{array}$ \\
\hline 800 & 0.44 & 1.40 & $0.03^{\S}$ & 0.001 & 9 \\
700 & 0.52 & 0.65 & 0.08 & 0.003 & 4 \\
600 & 0.56 & 0.25 & 0.30 & 0.01 & 1 \\
550 & 0.60 & 0.10 & 0.75 & 0.02 & 0.4 \\
500 & 0.62 & 0.30 & 3.50 & 0.03 & 0.09 \\
\hline
\end{tabular}

$\S$ Extrapolated value. 


\subsection{Theoretical}

\section{Defect Thermodynamics}

A model was developed that provides an analytical expression for the dependence of defect concentration on oxygen partial pressure. Unlike the traditional Brouwer approach, the expressions obtained are continuous across the entire defect equilibria. Consequently, it was possible to incorporate the effect of an electric potential on defect concentration at the gas-solid interface of SOFC electrolytes.

\section{Defect Transport}

A continuum level electrochemical model for defect transport in solid electrolytes was developed through solution of the well-known Nernst-Planck equation for flux. It has two major advances over previous models. First, it includes potential-dependent defect concentrations at the gas-solid interface rather than fixed defect concentrations. This feature allows the effect of variable loads on an SOFC to be accounted for.

Second, our model does not assume a linear potential distribution. Instead an equation for the electric potential is produced. It shows that the potential distribution approaches linearity in opencircuit conditions and for good electrolytes (negligible electronic conductivity and relatively high ionic conductivity).

Both features predict lower SOFC power and current efficiencies for mixed conducting electrolytes such as ceria. Which, by extrapolation, suggests that too much blame have been placed on the electrodes for shortfalls in the predicted performance of ceria-based SOFCs.

Finally, the model was tested, in open-circuit conditions, and it provided excellent fits to the experimental data.

\section{Bilayer Stability and Performance}

The models for defect thermodynamic and transport enabled the development of a model for the stability and performance of bilayer electrolytes and other bilayer combinations-e.g., anodeelectrolyte and electrolyte-cathode. The bilayer model shows how the stability of the bilayer (i.e., protecting the bismuth oxide layer from reduction) depends on the thickness ratio of the constituent layers. It further predicts how the bilayer's performance (ASR, defect current, etc.) is dependent on 
the thickness ratio. The model awaits experimental results for verification. This will be done in the future if funding can be secured.

\subsection{Forecast: Predictions and Projections}

The project has come to an end before we could complete all the desired tests on the bilayer. However, all our results thus far resonate the success of the bilayer concept as applied to ceria/bismuth oxide bilayers. At the beginning the project the primary questions to be answered were, "Can the high conductivity ceria/bismuth oxide bilayers be made?", "Will they be stable?", "Will secondary phases at the ceria-bismuth oxide interface degrade their performance?".

To answer these questions, we have shown that ceria/bismuth oxide bilayers can be made with an effective conductivity large enough to make them viable down to $500{ }^{\circ} \mathrm{C}$. We have also shown that stable bilayers can be made and that we can achieve near theoretical open-circuit potentials if the relative thickness of the bismuth oxide layer is large enough. Finally, we have also been able to show that there are no secondary interfacial phases present to degrade the performance of the bilayer.

With this in mind the obvious question to ask at the end of the project is whether the story is complete. The answer to that question is no, there is still much work to do. To fully establish the ceria/bismuth oxide bilayer as a viable candidate for future SOFCs, three main tasks remain to be completed. First, a suitable cathode must be found for the bilayer electrolyte. This is critical because this was one of the primary hurdles which prevented us from completing more performance tests on the bilayer. In fact, all further performance tests hinge on finding a suitable cathode. Second, the thickness ratio for the bilayer needs to be optimized experimentally and compared to the predictions of the bilayer model. Finally, the fabrication of the bilayers needs to be optimized for commercial application.

We see these final steps as crucial and challenging, but definitely doable. So the question is not whether they can be done, but if there is enough vision and support to get them done. 


\section{PRESENTATIONS AND PUBLICATIONS}

The following presentations and publications disseminated the research results supported under this contract.

\section{PUBLICATIONS:}

"Effect of Potential-Dependent Boundary Conditions and Non-Linear Potentials on Defect Distribution and Transport in n-Type Oxide Mixed Ionic-Electronic Conductors," K. L. Duncan and E. D. Wachsman, Journal of the Electrochemical Society, submitted.

"General Model for the Functional Dependence of Defect Concentration on Oxygen Potential in Mixed Conducting Oxides," K. L. Duncan and E. D. Wachsman, Solid State Ionics, submitted.

"Modeling Defect Generation, Ambipolar Transport and Association in n-Type Mixed Ionic-Electronic Conducting Oxides," K. L. Duncan and E. D. Wachsman, Journal of the Electrochemical Society, submitted.

"Effect of Oxygen Sublattice Order on Conductivity in Highly Defective Fluorite Oxides," E. D. Wachsman, Journal of the European Ceramic Society, accepted.

"Modeling the Performance and Stability of Bilayer Electrolytes for Low-Temperature Solid Oxide Fuel Cells," K.L. Duncan and E.D. Wachsman, Solid State Ionic Devices III, Electrochem. Soc., E.D. Wachsman, K.S. Lyons, M. Carolyn, F. Garzon, M. Liu, and J. Stetter, Ed., accepted.

"Stable High Conductivity Bilayered Electrolytes for Low Temperature Solid Oxide Fuel Cells," J.Y. Park and E.D. Wachsman, Solid Oxide Fuel Cells VIII, Electrochem. Soc., S.C. Singhal and M. Dokiya, Ed, 2003-07, 289-298 (2003).

"Designing for Thermochemical Applications," E.D. Wachsman, in An Introduction to Ceramic Engineering Design, D.E. Clark, D.C. Folz, and T.D. McGee, Ed., American Ceramic Society, 329346, ISBN 1-57498-131-5, 2002.

"Functionally Gradient Bilayer Oxide Membranes and Electrolytes," E. D. Wachsman, Solid State Ionics, 152-153, 657-662 (2002).

"A Higher Conductivity $\mathrm{Bi}_{2} \mathrm{O}_{3}$-Based Electrolyte," N. Jiang, E. D. Wachsman, and S. H. Jung, Solid State Ionics, 150, 347-353 (2002).

"Structural Stability and Conductivity of $\left(\mathrm{WO}_{3}\right)_{\mathrm{x}}\left(\mathrm{Dy}_{2} \mathrm{O}_{3}\right)_{\mathrm{y}}\left(\mathrm{Bi}_{2} \mathrm{O}_{3}\right)_{1-\mathrm{x}-\mathrm{y}}, " \mathrm{~S}$. H. Jung, E. D. Wachsman, and N. Jiang, Ionics, 8, 210-214 (2002).

"Effect of Dopant Polarizability on Oxygen Sublattice Order in Phase-Stabilized Cubic Bismuth Oxides," E. D. Wachsman, S. Boyapati, and N. Jiang, Ionics, 7, 1-6 (2001). 
"Effect of Oxygen Sublattice Ordering on Interstitial Transport Mechanism and Conductivity Activation Energies in Phase-Stabilized Cubic Bismuth Oxide," S. Boyapati, E. D. Wachsman, and N. Jiang, Solid State Ionics, 140, 149-160 (2001).

"Neutron Diffraction Study of Occupancy and Positional Order of Oxygen Ions in Phase-Stabilized Cubic Bismuth Oxides," S. Boyapati, E. D. Wachsman, and B. C. Chakoumakos, Solid State Ionics, 138, 293-304 (2001).

"A Model for the Spatial Distribution and Transport Properties of Defects in Mixed Ionic-Electronic Conductors: Part I Defect Concentration - Pressure Relationship," K. L. Duncan and E. D. Wachsman, Ionics, 6, 145-155 (2000).

"Modeling of Ordered Structures in Phase-Stabilized Cubic Bismuth Oxide," E. D. Wachsman, S. Boyapati, M. J. Kaufman, and N. Jiang, Journal of the American Ceramic Society, 83 [8], 1964-68 (2000).

\section{PRESENTATIONS}

"Stable Low Temperature Bilayer Solid Oxide Electrolytes Based on Unstable Materials," Materials Research Society, December 2-5, 2002, Boston, MA.

"Effect of Oxygen Sublattice Ordering on Conductivity in Highly Defective Fluorite Oxides," Plenary, $8^{\text {th }}$ International Conference on Electroceramics, August 25-28, 2002, Rome, Italy.

"Stable Low Temperature Bilayer Solid Oxide Electrolytes Based on Unstable Materials," University of Rome Tor-Vergata, August 23, 2002, Rome, Italy.

"Modeling the Electrochemical Performance and Thermo-Mechanical-Chemical Stability of Mixed Ionic-Electronic Conductors for Applications in SOFCs," National Energy Technology Laboratory, June 20, 2002, Morgantown, WV.

"Stable High Conductivity Bilayered Electrolytes for Low Temperature Solid Oxide Fuel Cells," US Department of Energy Workshop on Solid Oxide Fuel Cells, June 18-19, 2002, Pittsburgh, PA.

"Functionally Gradient Bilayer Oxide Membranes and Electrolytes," International Conference on Solid State Ionics, July 8-13, 2001, Cairns, Australia.

"Thermochemical Considerations in Engineering Design; Ceramic Fuel Cells, Membranes and Sensors," The 25th International Conference on Advanced Ceramics \& Composites, American Ceramic Society, January 21-26, 2001, Cocoa Beach, FL.

"Effect of Oxygen Sublattice Order on Conductivity in Phase-Stabilized Cubic Bismuth Oxides," 7th Euroconference on Ionics, October 1-7, 2000, Corsica, France.

"Effect of Oxygen Sublattice Ordering on Conductivity in Phase-Stabilized Cubic Bismuth Oxides," University of Rome Tor-Vergata, May 26, 2000, Rome, Italy.

"Effect of Oxygen Sublattice Ordering on Conductivity in Phase-Stabilized Cubic Bismuth Oxides," University of Missouri, May 4, 2000, Rolla, MO. 


\section{REFERENCES}

1. K. Mehta, S. J. Hong, J. -F. Jue and A. V. Virkar, in Solid Oxide Fuel Cells III, S. C. Singhal and H. Iwahara, ed., PV 93-4, p. 92, Electrochem. Soc. Proc. Series.

2. P. Soral, U. Pal and W. Worrell, J. Electrochem. Soc. 145 (1998) 99.

3. P. Shuk, H. -D. Wiemhöfer, U. Guth, W. Gopel and M. Greenblatt, Solid State Ionics 89 (1996) 179.

4. A. M. Azad, S. Larose and S. A. Akbar, J. Mat. Sci. 29 (1994) 4135.

5. E.D.Wachsman, P. Jayaweera, N. Jiang, D.M. Lowe, B.G. Pound, Journal of the Electrochemical Society,144 (1997) 233.

6. J. Van herle, T. Horita, T. Kawada, N. Sakai, H. Yokoko and M. Dokiya, Solid State Ionics, 86-88 (1996) 1255.

7. M. N. Rahaman, Ceramic Processing and Sintering, Marcel Decker, Inc., New York 1995.

8. B.C.H. Steele, Solid State Ionics, 129 (2000) 95.

9. A. V. Virkar, G. R. Miller, and R. S. Gordon, J. Am. Ceram. Soc., 61 (1978) 250.

10. S. J. Hong, K. Mehta, and A.V. Virkar, J. Electrochem. Soc., 145 (1998) 638.

11. C. Tian and S. Chan, Solid State Ionics, 134 (2000) 89.

12. R. Gerhardt and A. S. Nowick, J. Am. Ceram. Soc., 69 (1986) 641.

13. R. Gerhardt, A. S. Nowick, M. E. Mochel, and I. Dumler, J. Am. Ceram. Soc., 69 (1986) 647.

14. E. Schouler, G. Girond, M. Kleitz, J. Chem. Phys., 70 (1973).

15. G. M. Christie and F. P. F. van Berkel, Solid State Ionics, 83 (1996) 17.

16. P. L Chen and I. W. Chen, J. Am. Ceram. Soc., 79 (1996) 1793.

17. D. Y. Wang, D. S. Park, J. Griffith, and A. S. Nowick, Solid State Ionics, 2 (1981) 95.

18. T. Takahashi, H. Iwahara and Y. Nagai, J. Appl. Electrochem., 297 (1972)

19. T. Takahashi, and H. Iwahara, Mater. Res. Bull., 131447 (1978)

20. A. M. Azad, S. Larose, and S. A. Akbar, J. Mat. Sci., 294135 (1994)

21. P. Shuk, H. D. Wiemhofer, U. Guth, W. Gopel, and M. Greenblatt, Solid State Ionics, 89179 (1996)

22. L. G. Sillen, Ark. Kemi. Mineral. Geol. 12A 1 (1937).

23. V. G. Gattow and H. Schroder, Z. Anorg. Allg. Chem., 318197 (1962).

24. B. T. M. Willis, Acta Crystallogr. 1875 (1965).

25. P. D. Battle, C. R. A. Catlow, J. W. Heap, and L. M. Moroney, J. Solid State Chem., 638 (1986)

26. P. D. Battle, C. R. A. Catlow, J. W. Heap, and L. M. Moroney, J. Solid State Chem., 6742 (1987)

27. M. J. Verkerk, A. J. Burggraaf, Solid State Ionics, 3/4 463 (1981) 
28. M. J. Verkerk, G. M. H. Van De Velde, A. J. Burggraaf, and R. B. Helmholdt, J. Phys. Chem. Solids, 431129 (1982)

29. E. D. Wachsman, N. Jiang, D. M. Mason, and D. A. Stevenson, Proc. Electrochem. Soc., 89-11 15 (1989)

30. N Jiang, R. M. Buchanan, F. E. G. Henn, A. F. Marshall, D. A. Stevenson, and E. D. Wachsman, Mater. Res. Bull., 29, 247 (1994)

31. K. Z. Fung, J. Chen, and A. V. Virkar, J. Am. Ceram. Soc., 76, 2403 (1993)

32. E. D. Wachsman, S. Boyapati, M. J. Kaufman, and N. Jiang, Solid State Ionic Devices : Proc. Electrochem. Soc., 42 (1999)

33. E. D. Wachsman, S.Boyapati, M. J. Kaufman, and N. Jiang, J. Amer. Cer. Soc., 83 [8] 1964 (2000)

34. N. Jiang and E. D. Wachsman, J. Am. Ceram. Soc., 82, 3057 (1999)

35. S. Boyapati, E. D. Wachsman, and N. Jiang, Submitted to Solid State Ionics

36. A. C. Larson and R. B. Von Dreele, GSAS, General Structural and Analysis System, Report LAUR-86-748. Los Alamos National Laboratory, Los Alamos, NM

37. N. Jiang, R. M. Buchanan, D. A. Stevenson, W. D. Nix, Li Ji-Zhou, and Yang Ji-Lian, Materials Letters 22, 215 (1995)

38. N. Jiang, R. M. Buchanan, D. A. Stevenson, W. D. Nix, Li Ji-Zhou, and Yang Ji-Lian, Materials Letters 22, 215-219 (1995)

39. N. Jiang and E. D. Wachsman, J. Am. Ceram. Soc. 82, 3057-3064 (1999)

40. E. D. Wachsman, S. Boyapati, M. J. Kaufman, and N. Jiang, Solid State Ionic Devices: Proc. Electrochem. Soc. 99-13, 42-51 (1999)

41. V. G. Gattow and H. Schroder, Z. Anorg. Allg. Chem., 318197 (1962)

42. P. D. Battle, C. R. A. Catlow, J. W. Heap, and L. M. Moroney, J. Solid State Chem., 638 (1986)

43. P. D. Battle, C. R. A. Catlow, J. W. Heap, and L. M. Moroney, J. Solid State Chem., 6742 (1987)

44. L. G. Sillen, Ark. Kemi. Mineral. Geol. 12A 18 (1937)

45. B. T. M. Willis, Acta Crystallogr. 1875 (1965)

46. S. Boyapati, E. D. Wachsman, and B. C. Chakoumakos, Solid State Ionics, 138/3-4 293-304 (2001)

47. K. Koto, H. Schultz, and R. A. Huggins, Solid State Ionics, 5 355-365 (1980)

48. P. W. M. Jacobs and D. A. Mac Donaill, Solid State Ionics, 23 279-293 (1987)

49. P. W. M. Jacobs and D. A. Mac Donaill, Solid State Ionics, 23 295-305 (1987)

50. P. W. M. Jacobs and D. A. Mac Donaill, Solid State Ionics, 23 307-318 (1987)

51. E. D. Wachsman, S.Boyapati, M. J. Kaufman, and N. Jiang, J. Amer. Cer. Soc., 83 1964-68(2000).

52. K. Z. Fung, J. Chen, and A. V. Virkar, J. Am. Ceram. Soc 76, 2403-2418, (1993)

53. N.Jiang, Thesis, Stanford University

54. E. D. Wachsman, S.Boyapati, and N. Jiang, Ionics 7 (2001) 1. 
55. A. S. Nowick, W-K. Lee, and H. Jain, Solid State Ionics 28-30, 89-94 (1988)

56. P. D. Battle, C. R. A. Catlow, J. W. Heap and L. M. Moroney, J. Solid State Chem. 63 (1986) 8.

57. N. Jiang and E.D. Wachsman, J. Am. Ceram. Soc., 82 [11] (1999) 3057.

58. E. D. Wachsman, S. Boyapati, M. J. Kaufman, N. Jiang, J. Am. Ceram. Soc. 83 (2000) 1964.

59. E. D. Wachsman, N. Jiang, D. M. Mason and D. A. Stevenson, Proc. Electrochem., Soc., 89-11 (1989) 15.

60. S. Boyapati, E. D. Wachsman and B. C. Chakoumakos, Solid State Ionics 138 (2001) 293.

61. S. Boyapati, E. D. Wachsman and N. Jiang, Solid State Ionics, 140 (2001) 149.

62. E. D. Wachsman, S. Boyapati and N. Jiang, Ionics, 7 (2001) 1.

63. T. Takahashi and H. Iwahara, J. of Appl. Electrochem. 3 (1973) 65.

64. M. J. Verkerk and A. J. Burggraaf, J. Electrochem., 128 (1981) 75.

65. G. Meng, C. Chen, X. Han, P. Yang and D. Peng, J. Mat. Sci. 28-30 (1988) 533.

66. K. Z. Fung and A. V. Virkar, J. Am. Ceram. Soc., 74 [8] (1991) 1970.

67. L. Jizhou, Y. Jilian, L. Yuntao, N. Jiang, and H. Luo, Atomic Energy Science and Technology, Vol. 34, Suppl. Sep. (2000) 5.

68. S. Jung, E. D. Wachsman and N. Jiang, Ionics, accepted.

69. T. Takahashi, H. Iwahara and Y. Nagai, J. Appl. Electrochem., 2 97-104 (1972).

70. T. Takahashi, H. Iwahara, T. Arao, J. Appl. Electrochem., 5 187-195 (1975).

71. V. G. Gattow and H. Schroder, Z. Anorg. Allg. Chem., 318197 (1962).

72. L. G. Sillen, Ark. Kemi. Mineral. Geol. 12A 1 (1937).

73. B. T. M. Willis, Acta Crystallogr. 1875 (1965).

74. E. D. Wachsman, N. Jiang, D. M. Mason, and D. A. Stevenson, Proc. Electrochem. Soc., 89-11, 15 (1989).

75. M. J. Verkerk, A. J. Burggraaf, Solid State Ionics, 3/4 463-467 (1981).

76. E.D. Wachsman, G.R. Ball, N. Jiang, and D.A. Stevenson, Solid State Ionics, 52 213-218 (1992).

77. N. Jiang and E. D. Wachsman, J. Am. Ceram. Soc. 82, 3057-3064 (1999)

78. N Jiang, R. M. Buchanan, F. E. G. Henn, A. F. Marshall, D. A. Stevenson, and E. D. Wachsman, Mater. Res. Bull., 29, 247-254 (1994).

79. N. Jiang, R. M. Buchanan, D. A. Stevenson, W. D. Nix, Li Ji-Zhou, and Yang Ji-Lian, Materials Letters 22, 215-219 (1995).

80. E. D. Wachsman, S.Boyapati, M. J. Kaufman, and N. Jiang, J. Amer. Cer. Soc., 83, 1964-68 (2000).

81. E. D. Wachsman, S. Boyapati, M. J. Kaufman, and N. Jiang, "Solid State Ionic Devices", Proc. Electrochem. Soc. 99-13, 42-51 (1999).

82. S. Boyapati, E. D. Wachsman, and B. C. Chakoumakos, Solid State Ionics, 138/3-4, 293 (2001).

83. D. Steele and B.E.F. Fender, The structure of cubic $\mathrm{ZrO}_{2}: \mathrm{YO}_{1.5}$, J. Phys. C, 1974, 7, 1-11. 
84. Nakamura and J.B. Wagner, J. Electrochem. Soc., 1986, 133, 1542-1548.

85. W. L. Roth, R. Wong, A. I. Goldman, E. Canova, Y. H. Kao, and B. Dunn, Solid State Ionics, 1986, 18-19, 1115-1119.

86. P. Li, I-W. Chen, and J. E. Penner-Hahn, J. Amer. Ceram. Soc., 1994, 77, 118-28.

87. J.P. Goff, W. Hayes, S. Hull, M.T. Hutchings, and K.N. Clausen, Phys. Rev. B, 1999, 59, 14202.

88. F. Shimojo, T. Okabe, F. Tachibana, M. Kobayashi, and H. Okazaki, J. Phys. Soc. Jpn., 1992, 61, 2848.

89. A. Bogicevic, C. Wolverton, G.M. Crosbie, and E.B. Stechel, Phys. Rev. B, 2001, 64, 0141061.

90. E. D. Wachsman, N. Jiang, D. M. Mason, and D. A. Stevenson, Proc. Electrochem. Soc., 1989, 89-11, 15.

91. E. D. Wachsman, G.R. Ball, N. Jiang, and D.A. Stevenson, Solid State Ionics, 1992, 52213.

92. N. Jiang and E. D. Wachsman, J. Am. Ceram. Soc., 1999, 82, 3057-3064.

93. N Jiang, R. M. Buchanan, F. E. G. Henn, A. F. Marshall, D. A. Stevenson, and E. D. Wachsman, Mater. Res. Bull., 1994, 29, 247-254.

94. E. D. Wachsman, S. Boyapati, M. J. Kaufman, and N. Jiang, J. Amer. Cer. Soc., 2000, 83, 1964.

95. E. D. Wachsman, S. Boyapati, M. J. Kaufman, and N. Jiang, Proc. Electrochem. Soc., 1999, 99 13, 42-51.

96. N. Jiang, R. M. Buchanan, D. A. Stevenson, W. D. Nix, Li Ji-Zhou, and Yang Ji-Lian, Mater. Lett., 1995, 22, 215-219.

97. S. Boyapati, E. D. Wachsman, and B. C. Chakoumakos, Solid State Ionics, 2001, 138/3-4, 293

98. E. D. Wachsman, S. Boyapati, and N. Jiang, Ionics, 2001, 7, 1-6.

99. S. Boyapati, E. D. Wachsman, and N. Jiang, Solid State Ionics, 2001, 140, 149-160.

100.N. Jiang, E. D. Wachsman, and S.H. Jung, Solid State Ionics, 2002, 150, 347-353.

101.S. H. Jung, E. D. Wachsman, and N. Jiang, Ionics, 2002, 8, 210-214.

102. Y. Iwadate, K. Shirao, and K. Fukushima, J. Alloys and Compounds, 1999, 284 89-91.

103.H. Arai, in Proceedings of the International Syposium on SOFCs, Nov. 13-14, 1989, Nagoya, Japan, eds. O. Yamamoto, M. Dokiya, and H. Tagawa, Science House, Tokyo, Japan (1989) 12.

104.K. Tsukamoto, F. Uchiyama, Y. Kaga, Y. Ohno, T. Yanagisawa, A. Monma, Y. Takahagi, M. J. Lain, and T. Nakajima, Solid State Ionics, 40-41 (1990) 1003.

105. T. Setoguchi, M. Sawano, E. Eguchi, and H. Arai, Solid State Ionics, 40-41 (1990) 502.

106.H. Arai, in Proceedings of the International Syposium on SOFCs, Nov. 13-14, 1989, Nagoya, Japan, eds. O. Yamamoto, M. Dokiya, and H. Tagawa, Science House, Tokyo, Japan (1989) 58.

107.M. F. Carolan and J. N. Michaels, Solid State Ionics, 25 (1987) 207

108. N. Nicoloso, B. Leibold, and H. U. Habermeier, in Laser Ablation of Electronic Materials, eds. E. Fogarassy and S. Lazare, Elsevier, Amsterdam, The Netherlands (1992) 385

109.S. Souza, S. J. Visco, and L. C. De Jonghe, J. Electrochem. Soc., 144 (1997) L35 
110.J. Will, A. Mitterdorfer, C. Kleinlogel, D. Perednis, and L. J. Gauckler, Solid State Ionics, 131 (2000) 79

111. T. L. Clites, M.D. Thesis, Development of Stable Mixed-Conducting Bilayer Perovskite Membranes for the Direct Conversion of Methane to Syngas, University of Florida, 1999

112. E.D.Wachsman, P. Jayaweera, N. Jiang, D.M. Lowe, B.G. Pound, J. Electrocchem. Soc.,144 (1997) 233.

113. S. Ohara, R. Maric, X. Zhang, K. Mukai, T. Fukui, H. Yoshida, T. Inagaki, K. Miura, Journal of Power Sources, 86 (2000) 455.

114. J. Will, A. Mitterdorfer, C. Kleinlogel, D. Perednis, L.J. Gaukler, Solid State Ionics, 131 (2000) 79.

115. S. deSouza, S.J. Visco, L.C. DeJonghe, Journal of the Electrochemical Society, 144 (1997) L35.

116. L. W. Tai, M. M. Nasrallah, H. U. Anderson, in : S. C. Singhal, H. Iwahara (Eds.), Proc. $3^{\text {rd }}$ Int. Symp. Solid Oxide Fuel Cells, The Electrochemical Society Proceeding Series (1999) p. 241, Pennington, NJ.

117. L. W. Tai, M. M. Nasrallah, H. U. Anderson, M. A. Alim, J. Electrochem. Soc., 142 (1995) 491.

118. L. W. Tai, M. M. Nasrallah, H. U. Anderson, D. M. Sparlin, S. R. Sehlin, Solid State Ionics, 76 (1995) 273.

119. K. Zheng, B.C.H. Steele, M. Sahibzada, I.S. Metcalfe, Solid State Ionics, 86-88 (1996)1241.

120. R. Doshi, V. L. Richards, J. D. Carter, X. Wang, and M. Krumpelt, J. Electrochem. Soc. 146 (1999) 1273

121. N. S. Choudury and J. W. Patterson, J. Electrochem. Soc. 1181384 (1971).

122. I. Riess, Solid State Ionics 52127 (1992).

123. M. Liu, J. Electrochem. Soc. 1441813 (1997).

124. S. Yuan and U. Pal, J. Electrochem. Soc. 1433214 (1996).

125. J. Newman, Electrochemical Systems, ch. 2 and ch. 11, Prentice-Hall, NJ (1991).

126. H. J. Bouwmeester and P. J. Gellings, Ch. 1 CRC Handbook of Solid State Electrochemistry

127. K. L. Duncan and E. D. Wachsman, J. Electrochem. Soc., submitted.

128. K. L. Duncan and E. D. Wachsman, Solid State Ionics, submitted.

129. H. L. Tuller, Nonstoichiometric Oxides, O. Sorensen, Editor, p. 271, Academic, New York, (1981).

130. K. Eguchi, T. Setoguchi, T. Inoue and H. Arai, Solid State Ionics 52265 (1992).

131. T. Kobayashi, S. Wang, M. Dokiya, Hiroaki T. and T. Hashimoto, Solid State Ionics 126349 (1999).

132. H. Inaba and H. Tagawa, Solid State Ionics 831 (1996).

133. R. Doshi, V. L. Richards, J. D. Carter, X. Wang and M. Krumpelt, J. Electrochem. Soc. 146 1273 (1999).

134. I. Riess, M. Godickemeier and L. Gauckler, Solid State Ionics 9091 (1996). 
135. E. Perry Murray and S. A. Barnett, Solid State Ionics 143265 (2001).

136. K. Duncan and E. Wachsman, Proceedings of the Symposium on Design and Mathematical Modeling of Electrochemical Systems, Electrochem. Soc. Proc. Series (2001).

137. B.C.H. Steele, Solid State Ionics, 75 (1995) 157.

138. E. Wachsman and K. Duncan, Proceedings of the International Symposium on Solid State Ionic Devices, Vol. 99-13 (The Electrochemical Society, Inc. 1999), p. 159.

139. M. Liu, J. Electrochem. Soc. 1441813 (1997).

140. K. Duncan, Ph. D. Thesis, University of Florida (2001).

141. J. Newman, Electrochemical Systems (Prentice-Hall, 1991).

142. H. Bouwmeester and P. Gellings, Ch. 1 CRC Handbook of Solid State Electrochemistry

143. T. Takahashi, H. Iwahara and T. Arao, J. App. Electrochem. 7 (1977) 299.

144. M. Verkerk, K. Keizer and A. Burggraaf, J. App. Electrochem. 10 (1980) 81.

145. B.C.H. Steele, Solid State Ionics, 129 (2000) 95.

146. P. Shuk, H. -D. Wiemhöfer, U. Guth, W. Gopel and M. Greenblatt, Solid State Ionics 89 (1996) 179.

147. E. Wachsman, P. Jayaweera, N. Jiang, B. Pound, J. Electrochem. Soc., 144 (1997) 233.

148. C. Xia, Y. Zhang, M. Liu, App. Phys. Lett. 82 (2003) 901.

149. J. Liu and S. A. Barnet, Solid State Ionics, 158, (2003) 11.

150. M. Godickemeier, K. Sasaki, L. Gauckler and I. Riess, J. Electrochem. Soc. 144 (1997) 1635.

151. M. Godickemeier and L. Gauckler, J. Electrochem. Soc. 145 (1998) 414. 